\title{
Actinide-Catalyzed Intermolecular Addition of Alcohols to Carbodiimides
}

\author{
Rami J. Batrice, ${ }^{\dagger}$ Christos E. Kefalidis, ${ }^{\ddagger}$ Laurent Maron, ${ }^{\ddagger}$ Moris S. Eisen ${ }^{\dagger, *}$ \\ †Schulich Faculty of Chemistry, Technion - Israel Institute of Technology, Haifa 3200008, Israel. \\ ¥LPCNO, CNRS, \& INSA, Université Paul Sabatier, 135 Avenue de Rangueil, Toulouse 31077, France. \\ *E-mail: chmoris@tx.technion.ac.il
}

\section{Supplementary Information}

\section{Experimental Section}

All manipulations of air-sensitive materials were performed with the rigorous exclusion of oxygen and moisture in flamed Schlenk-type glassware or J-Young Teflon valve-sealed NMR tubes on a dual manifold Schlenk line interfaced to a high vacuum ( $10^{-5}$ Torr) line, or in a nitrogen-filled Innovative Technologies glovebox with a medium-capacity recirculator (1-2 ppm of $\mathrm{O}_{2}$ ). Argon and nitrogen were purified by passage through $\mathrm{MnO}$ oxygen-removal column and a Davison $4 \AA$ molecular sieve column. Hydrocarbon solvents benzene- $d_{6}$ (Cambridge Isotopes), toluene (Bio-Lab), and diethyl ether (BioLab) were distilled under vacuum from $\mathrm{Na} / \mathrm{K}$ alloy. 1,3-diisopropylcarbodiimide was distilled from sodium bicarbonate under nitrogen atmosphere, and 1,3-di-p-tolylcarbodiimide was dried under vacuum $\left(10^{-6}\right)$ for 12 hours on a high vacuum line. Methanol and ethanol were dried using sodium ( $\left.\mathrm{Na}\right)$ metal, distilled, and stored over 4 Å molecular sieves. Isopropanol and tert-butanol were refluxed over $\mathrm{CaH}_{2}$, distilled, and stored over $4 \AA$ molecular sieves. The actinide complexes $\mathrm{U}\left[\mathrm{N}\left(\mathrm{SiMe}_{3}\right)_{2}\right]_{3}(\mathbf{1})^{1}$ and $\left[\left(\mathrm{Me}_{3} \mathrm{Si}\right)_{2} \mathrm{~N}\right]_{2} \mathrm{An}\left[\kappa^{2}-(\mathrm{N}, \mathrm{C})-\mathrm{CH}_{2} \mathrm{Si}\left(\mathrm{CH}_{3}\right)_{2} \mathrm{~N}\left(\mathrm{SiMe}_{3}\right)\right](\mathrm{An}=\mathrm{U}(2) \text {, Th (3) })^{2}$ were prepared according to published procedures. All the aforementioned reagents were stored in an inert atmosphere glovebox prior to use. $O$-deuterated alcohols were prepared by quenching the corresponding metal-alkoxide ( $\mathrm{NaOEt}$ or $\mathrm{KO}^{t} \mathrm{Bu}$ ) with excess $\mathrm{D}_{2} \mathrm{O}$ at reduced temperature, followed by fractional distillation, degassing, and storing over 4 Å molecular sieves.

NMR spectra were recorded on Bruker Avance 300, Bruker Avance III 400, or Bruker Avance 500 spectrometers on crude reaction mixtures. Chemical shifts for ${ }^{1} \mathrm{H}$ and ${ }^{13} \mathrm{C}$ NMR are referenced to internal protiosolvent and reported relative to tetramethylsilane. J-values are reported for ${ }^{1} \mathrm{H} N M R$ coupling constants in the unit of Hertz $(\mathrm{Hz})$.

\section{General Procedure for the Catalytic Addition of Alcohols to Carbodiimides}

In a typical experiment, approximately $5 \mathrm{mg}$ of desired catalyst in $600 \mu \mathrm{L}$ of $\mathrm{C}_{6} \mathrm{D}_{6}$ was transferred to a J. Young Teflon sealed NMR tube, followed by the addition of carbodiimide (100 equiv.) and alcohol (100 equiv.). Samples were then placed in an oil bath preheated to $75{ }^{\circ} \mathrm{C}$, and the reaction progress monitored at regular intervals using ${ }^{1} \mathrm{H}$ NMR spectroscopy for up to 24 hours. After completion of the reaction, crude mixtures were analysed using ${ }^{1} \mathrm{H},{ }^{13} \mathrm{C}$, and $2 \mathrm{D}$ NMR (when needed) spectroscopy as well as mass spectrometry. Known compounds were compared to previous literature.

\section{Characterization Data}

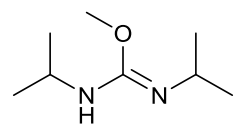

$N, N^{\prime}$-diisopropyl-O-methylisourea: The insertion of methanol (5.7 $\left.\mu \mathrm{L}, 140 \mu \mathrm{mol}\right)$ into diisopropylcarbodiimide $(21.9 \mu \mathrm{L}, 140 \mu \mathrm{mol})$ was carried out following the general procedure described. ${ }^{1} \mathrm{H}$ NMR $\left(300 \mathrm{MHz}, \mathrm{C}_{6} \mathrm{D}_{6}\right) \delta 3.80-3.68(\mathrm{~m}, 1 \mathrm{H}), 3.66(\mathrm{~s}, 3 \mathrm{H})$, $3.11(\mathrm{~m}, 1 \mathrm{H}), 2.35$ (bs, $1 \mathrm{H}), 1.22(\mathrm{~d}, J=6.2 \mathrm{~Hz}, 6 \mathrm{H}), 0.90$ (d, $J=6.4 \mathrm{~Hz}, 6 \mathrm{H}) .{ }^{13} \mathrm{C} \mathrm{NMR}\left(101 \mathrm{MHz}, \mathrm{C}_{6} \mathrm{D}_{6}\right) \delta$ 151.97, 52.56, 50.09, 46.56, 43.49, 23.92. MS (APCI+): $m / z 159.1495(M+H) *$ 


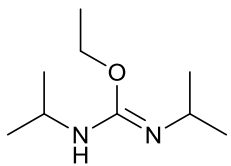

$\boldsymbol{N}, \boldsymbol{N}^{\prime}$-diisopropyl-O-ethylisourea: The insertion of ethanol $(8.2 \mu \mathrm{L}, 140 \mu \mathrm{mol})$ into diisopropylcarbodiimide $(21.9 \mu \mathrm{L}, 140 \mu \mathrm{mol})$ was carried out following the general procedure described. ${ }^{1} \mathrm{H}$ NMR $\left(400 \mathrm{MHz}, \mathrm{CDCl}_{3}\right) \delta 4.07(\mathrm{q}, J=7.1 \mathrm{~Hz}, 2 \mathrm{H}), 3.83-3.71$ (m, 1H), $3.40(\mathrm{bs}, 1 \mathrm{H}), 3.21-3.09(\mathrm{~m}, 1 \mathrm{H}), 1.24(\mathrm{t}, J=7.0 \mathrm{~Hz}, 3 \mathrm{H}), 1.12(\mathrm{~d}, J=6.4 \mathrm{~Hz}$, $6 \mathrm{H}), 1.09(\mathrm{~d}, J=6.4 \mathrm{~Hz}, 6 \mathrm{H}) .{ }^{13} \mathrm{C} \mathrm{NMR}\left(101 \mathrm{MHz}, \mathrm{CDCl}_{3}\right) \delta 151.92,60.78,46.28,43.42,24.47,24.06$, 14.53.MS (APCl+): $\mathrm{m} / \mathrm{z} 173.1658(\mathrm{M}+\mathrm{H})^{*}$

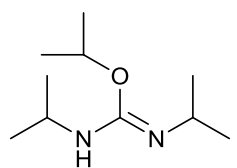

$\boldsymbol{N}, N^{\prime}$-diisopropyl-O-isopropylisourea: The insertion of isopropanol $(10.7 \mu \mathrm{L}, 140$ $\mu \mathrm{mol})$ into diisopropylcarbodiimide $(21.9 \mu \mathrm{L}, 140 \mu \mathrm{mol})$ was carried out following the general procedure described. ${ }^{1} \mathrm{H}$ NMR $\left(300 \mathrm{MHz}, \mathrm{C}_{6} \mathrm{D}_{6}\right) \delta 5.33$ (hept, $J=6.2 \mathrm{~Hz}, 1 \mathrm{H}$ ), $3.86-3.60(\mathrm{~m}, 1 \mathrm{H}), 3.20$ (bs, $1 \mathrm{H}), 3.13(\mathrm{~m}, 1 \mathrm{H}), 1.21(\mathrm{~d}, J=6.2 \mathrm{~Hz}, 12 \mathrm{H}), 0.92(\mathrm{~d}, J=$ $6.4 \mathrm{~Hz}, 6 \mathrm{H}) .{ }^{13} \mathrm{C}$ NMR $\left(101 \mathrm{MHz}, \mathrm{C}_{6} \mathrm{D}_{6}\right) \delta 150.40,66.58,46.52,43.33,24.91,23.92,22.20 . \mathrm{MS}(\mathrm{ESI}+)$ : $\mathrm{m} / \mathrm{z} 187.1850(\mathrm{M}+\mathrm{H})^{*}$

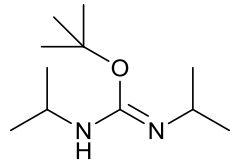

$\boldsymbol{N}, \boldsymbol{N}^{\prime}$-diisopropyl-O-tert-butylisourea: ${ }^{3}$ The insertion of isopropanol (13.3 $\mu \mathrm{L}, 140$ $\mu \mathrm{mol})$ into diisopropylcarbodiimide $(21.9 \mu \mathrm{L}, 140 \mu \mathrm{mol})$ was carried out following the general procedure described. ${ }^{1} \mathrm{H}$ NMR $\left(300 \mathrm{MHz}, \mathrm{C}_{6} \mathrm{D}_{6}\right) \delta 5.55$ (bs, $\left.1 \mathrm{H}\right), 3.75-3.58$ $(\mathrm{m}, 1 \mathrm{H}), 3.18-3.04(\mathrm{~m}, 1 \mathrm{H}), 1.57(\mathrm{~s}, 9 \mathrm{H}), 1.17(\mathrm{~d}, J=6.4 \mathrm{~Hz}, 6 \mathrm{H}), 0.92(\mathrm{~d}, J=6.4 \mathrm{~Hz}$, 6H). ${ }^{13} \mathrm{C}$ NMR $\left(101 \mathrm{MHz}, \mathrm{C}_{6} \mathrm{D}_{6}\right) \delta 154.70,72.75,48.18,40.20,29.62,22.92,20.69 . \mathrm{MS}(\mathrm{APCl}+): \mathrm{m} / \mathrm{z}$ $201.1962(M+H)^{*}$

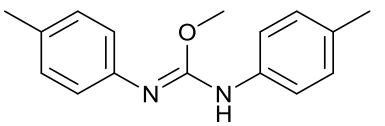

$\boldsymbol{N}, \boldsymbol{N}^{\prime}$-di-p-tolyl-O-methylisourea: The insertion of methanol $(8.5 \mu \mathrm{L}, 209$ $\mu \mathrm{mol})$ into di-p-tolylcarbodiimide $(46.4 \mathrm{mg}, 209 \mu \mathrm{mol})$ was carried out following the general procedure described. See Figure S1. ${ }^{1} \mathrm{H}$ NMR (500 $\left.\mathrm{MHz}, \mathrm{C}_{6} \mathrm{D}_{6}\right) \delta 7.03(\mathrm{~m}, 4 \mathrm{H}), 6.84(\mathrm{~m}, 2 \mathrm{H}), 6.74(\mathrm{~m}, 2 \mathrm{H}), 5.87(\mathrm{bs}, 1 \mathrm{H}), 3.74(\mathrm{~s}, 3 \mathrm{H}), 2.17(\mathrm{~s}, 3 \mathrm{H}), 2.06(\mathrm{~s}$, $3 \mathrm{H}) .{ }^{13} \mathrm{C}$ NMR $\left(126 \mathrm{MHz}, \mathrm{C}_{6} \mathrm{D}_{6}\right) \delta 150.74,146.37,136.68,132.49,132.07,130.70,129.56,122.94$, 121.10, 53.66, 20.86, 20.68. MS (APCl+): $\mathrm{m} / \mathrm{z} 255.1507(\mathrm{M}+\mathrm{H}) *$

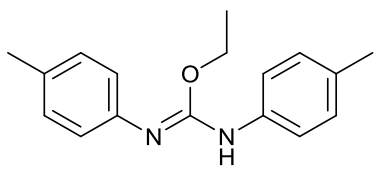

$\boldsymbol{N}, \boldsymbol{N}^{\prime}$-di-p-tolyl-O-ethylisourea: The insertion of ethanol (12.2 $\mu \mathrm{L}, 209$ $\mu \mathrm{mol})$ into di-p-tolylcarbodiimide $(46.4 \mathrm{mg}, 209 \mu \mathrm{mol})$ was carried out following the general procedure described. ${ }^{1} \mathrm{H} \mathrm{NMR}\left(300 \mathrm{MHz}, \mathrm{C}_{6} \mathrm{D}_{6}\right) \delta 7.06$ $-7.00(\mathrm{~m}, 4 \mathrm{H}), 6.88-6.74(\mathrm{~m}, 4 \mathrm{H}), 5.88(\mathrm{bs}, 1 \mathrm{H}), 4.37(\mathrm{q}, J=7.1 \mathrm{~Hz}, 2 \mathrm{H})$, $2.17(\mathrm{~s}, 3 \mathrm{H}), 2.05(\mathrm{~s}, 3 \mathrm{H}), 1.13(\mathrm{t}, J=7.1 \mathrm{~Hz}, 3 \mathrm{H}) .{ }^{13} \mathrm{C}$ NMR $\left(126 \mathrm{MHz}, \mathrm{C}_{6} \mathrm{D}_{6}\right) \delta 150.15,146.51,136.86$, $132.28,131.98,130.70,129.56,122.97,120.90,62.69,20.86,20.69,14.39$. MS (APCl+): m/z 269.1668 $(\mathrm{M}+\mathrm{H})^{*}$ 
<smiles>Cc1ccc(N=C(Nc2ccc(C)cc2)OC(C)C)cc1</smiles>

$\boldsymbol{N}, \boldsymbol{N}^{\prime}$-di-p-tolyl-O-isopropylisourea: The insertion of isopropanol $(16.0 \mu \mathrm{L}$, $209 \mu \mathrm{mol}$ ) into di-p-tolylcarbodiimide (46.4 mg, $209 \mu \mathrm{mol})$ was carried out following the general procedure described. ${ }^{1} \mathrm{H} N M R\left(500 \mathrm{MHz}, \mathrm{C}_{6} \mathrm{D}_{6}\right) \delta 7.03$ $(\mathrm{d}, J=7.9 \mathrm{~Hz}, 1 \mathrm{H}), 6.87-6.75(\mathrm{~m}, 1 \mathrm{H}), 5.89(\mathrm{~s}, 1 \mathrm{H}), 5.54-5.44(\mathrm{~m}, 1 \mathrm{H}), 2.16$ (s, 1H), $2.05(\mathrm{~s}, 1 \mathrm{H}), 1.26(\mathrm{~d}, J=6.2 \mathrm{~Hz}, 2 \mathrm{H}) .{ }^{13} \mathrm{C}$ NMR $\left(126 \mathrm{MHz}, \mathrm{C}_{6} \mathrm{D}_{6}\right) \delta$ 149.60, 146.61, 136.98, 132.15, 131.90, 130.69, 129.55, 122.96, 120.82, 69.64, 21.99, 20.86, 20.68. MS (APCl+): m/z 283.1808 (M + $\mathrm{H})^{*}$

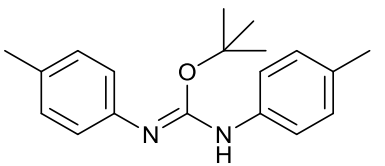

$\boldsymbol{N}, N^{\prime}$-di-p-tolyl-O-tert-butylisourea: The insertion of tert-butanol $(33.2 \mu \mathrm{L}$, $350 \mu \mathrm{mol})$ and 1,3-di- $p$-tolylcarbodiimide (55 $\mu \mathrm{L}, 350 \mu \mathrm{mol})$ was carried out following the general procedure described. $\delta_{H}\left(300 \mathrm{MHz}, \mathrm{C}_{6} \mathrm{D}_{6}\right) 7.11-6.92$ $(4 \mathrm{H}, \mathrm{m}), 6.90-6.73(4 \mathrm{H}, \mathrm{m}), 5.85(1 \mathrm{H}, \mathrm{bs}), 2.17(3 \mathrm{H}, \mathrm{s}), 2.06(3 \mathrm{H}, \mathrm{s}), 1.60$ $(9 \mathrm{H}, \mathrm{s}) .{ }^{13} \mathrm{C}$ NMR $\left(75 \mathrm{MHz}, \mathrm{C}_{6} \mathrm{D}_{6}\right) \delta 148.64,145.08,131.83,130.12,129.66,122.79,120.98,120.79,118.65$, 81.26, 28.51, 28.40, 20.79. MS (APCI+): $\mathrm{m} / \mathrm{z} 295.0716(\mathrm{M}+\mathrm{H})$ *

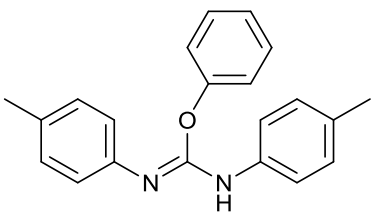

$\boldsymbol{N}, \boldsymbol{N}^{\prime}$-di-p-tolyl-O-phenylisourea: The insertion of phenol $(19.7 \mathrm{mg}, 350$ $\mu \mathrm{mol}$ ) and 1,3-di-p-tolylcarbodiimide (55 $\mu \mathrm{L}, 350 \mu \mathrm{mol})$ was carried out following the general procedure described. ${ }^{1} \mathrm{H} N M R\left(500 \mathrm{MHz}, \mathrm{C}_{6} \mathrm{D}_{6}\right) \delta 7.26$ (bs, 1H), $7.02(\mathrm{~m}, 4 \mathrm{H}), 6.81(\mathrm{~d}, J=8.0 \mathrm{~Hz}, 4 \mathrm{H}), 6.75(\mathrm{~m}, 1 \mathrm{H}), 6.67(\mathrm{~m}, 2 \mathrm{H}), 6.63(\mathrm{~d}, J$ $=8.0 \mathrm{~Hz}, 2 \mathrm{H}), 2.01(\mathrm{~s}, 3 \mathrm{H}), 1.96(\mathrm{~s}, 3 \mathrm{H}) .{ }^{13} \mathrm{C} N M R\left(126 \mathrm{MHz}, \mathrm{C}_{6} \mathrm{D}_{6}\right) \delta$ 156.99, 144.83, 144.10, 136.96, 131.83, 129.68, 129.46, 129.11, 122.37, 121.81, 120.42, 120.17, 115.75, 20.86, 20.78. MS (ESI+): $\mathrm{m} / \mathrm{z} 317.1604(\mathrm{M}+\mathrm{H})^{*}$ 


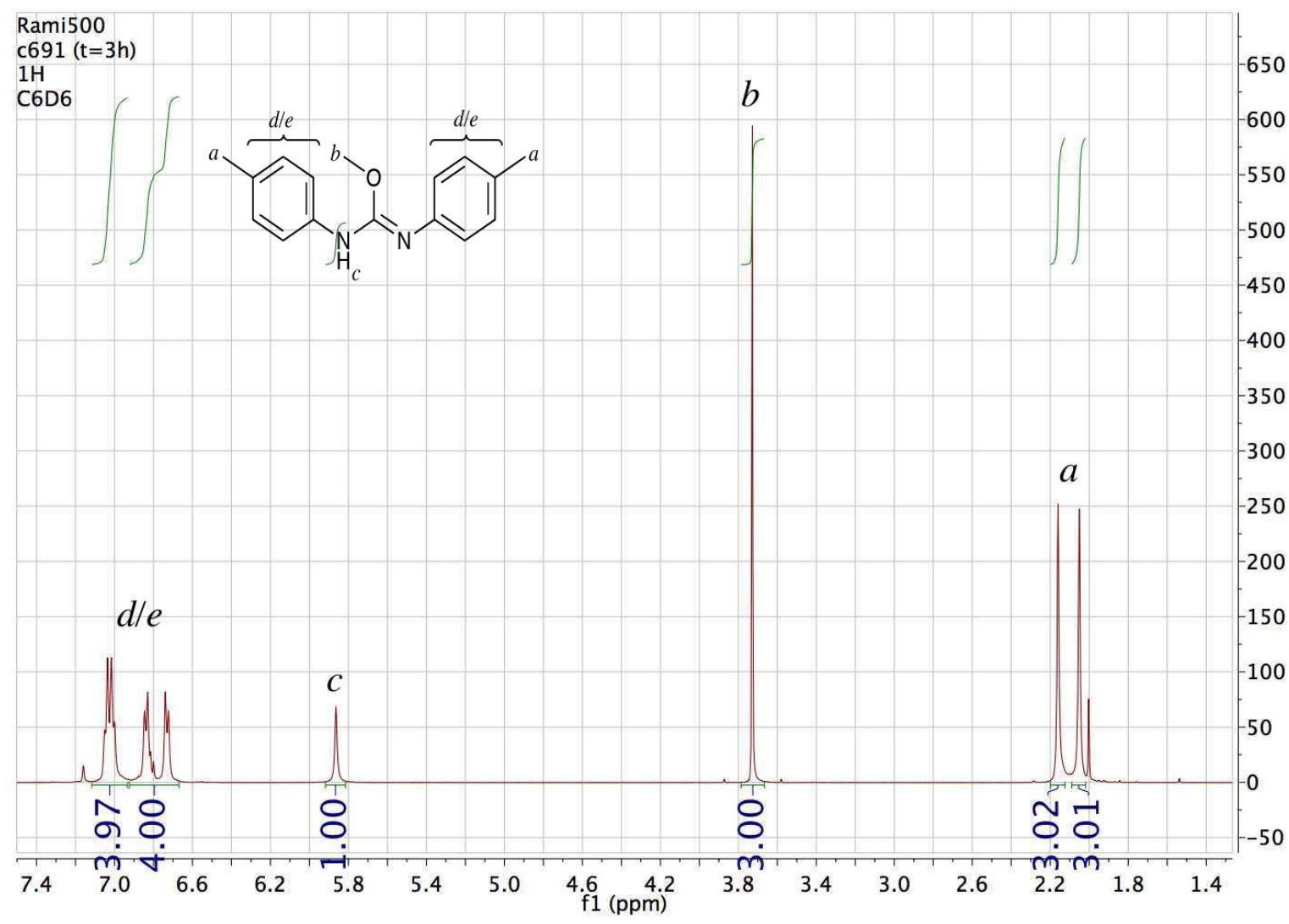

Figure S1: Representative sample of actinide-catalyzed addition of alcohols to carbodiimides. Reaction shown is thorium (3) mediated addition of methanol to 1,3-di-p-tolylcarbodiimide.

Preparative scale reaction of tert-butanol insertion into 1,3-di-p-tolylcarbodiimide

In an inert atmosphere glovebox, a Schlenk tube with stir bar and Teflon stopper was added $0.50 \mathrm{~g}$ 1,3-di-p-tolylcarbodiimide ( $2.25 \mathrm{mmol}$ ). A solution of catalyst 3 (30 mg, $42.1 \mu \mathrm{mol}, 2 \mathrm{~mol} \%$ ) in $10 \mathrm{~mL}$ of toluene was added to the solids, followed by the immediate addition of $215 \mu \mathrm{L}$ tert-butanol (167 $\mathrm{mg}, 2.25 \mathrm{mmol})$. The tube was sealed, removed from the glovebox, and immediately placed in an oil bath preheated to $75{ }^{\circ} \mathrm{C}$ with stirring for 24 hours. After completion of the reaction, the solvent was removed in vacuo. A yellow viscid liquid remained which was dissolved in $10 \mathrm{~mL}$ dichloromethane, filtered through a plug of Celite to remove the catalyst, loaded in a separatory funnel with $20 \mathrm{~mL}$ of distilled water, and the organics separated then evaporated to dryness. The oil was then dissolved in $n$-hexane, filtered through a plug of Celite, and evaporated under vacuum, leaving a white waxy solid ( $0.56 \mathrm{~g}, 85 \%$ isolated yield).

\section{Activation Studies of Precatalysts (1-3)}

In an inert atmosphere glovebox, a stock solution of the respective precatalyst was prepared in $\mathrm{C}_{6} \mathrm{D}_{6}$. An aliquot of the stock solution was transferred to a J-Young Teflon valve-sealed NMR tube, followed by the addition of four equivalents of alcohol. The tube was then sealed, shaken, and heated to $75^{\circ} \mathrm{C}$ for 20 minutes, followed by acquiring a ${ }^{1} \mathrm{H}$ NMR spectrum of the mixture. It was revealed that complete displacement of the amides occurs to form an actinide-alkoxide complex. Attempts to isolate and crystallize these complexes was unsuccessful. In an attempt to elucidate the identity of precatalyst (1) after activation, four equivalents of diisopropylcarbodiimide was added, followed by heating and acquisition of the NMR spectrum (Figure S2). 


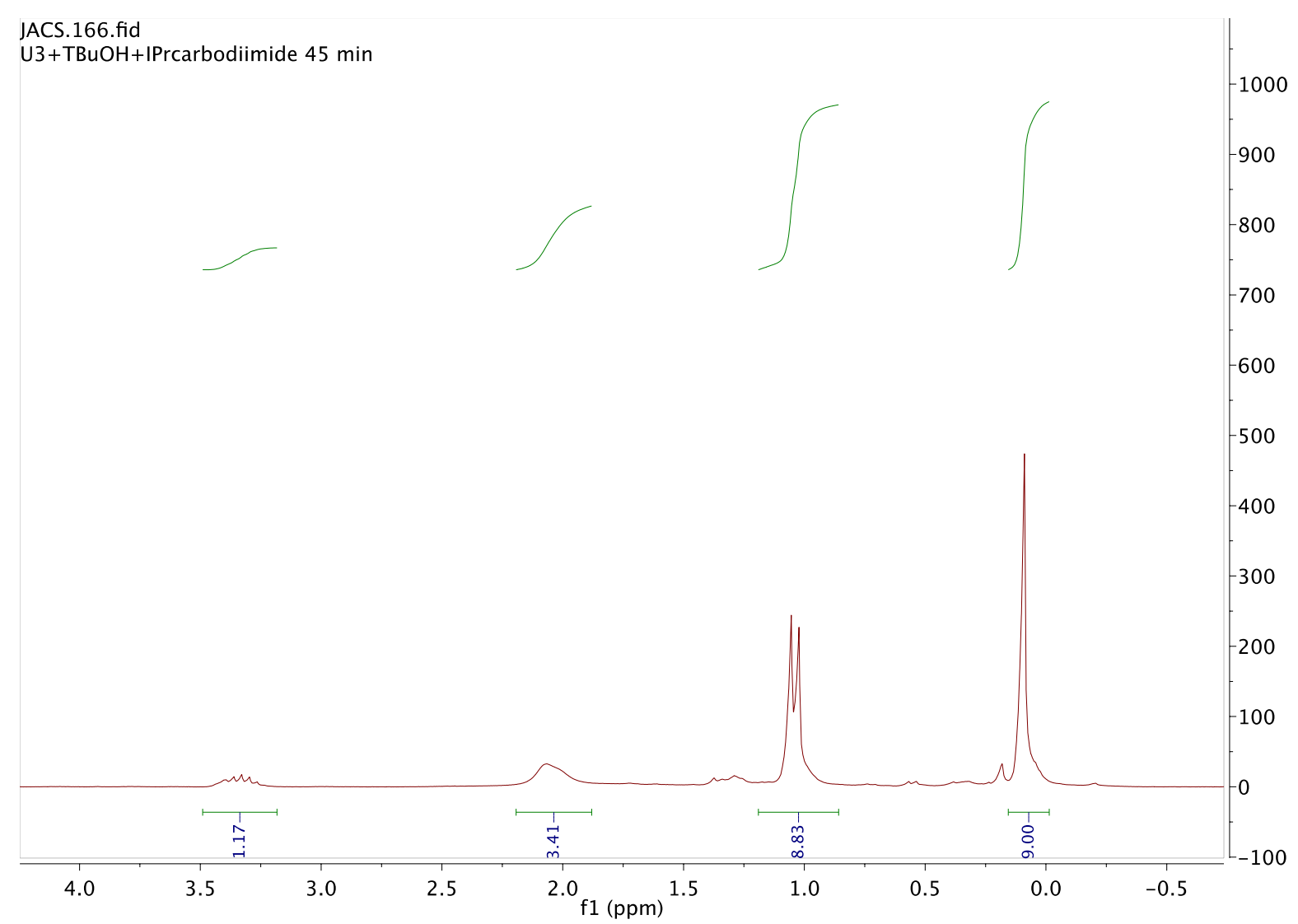

Figure S2: ${ }^{1} \mathrm{H}$ NMR spectrum of complex 1 after addition of ${ }^{\mathrm{t}} \mathrm{BuOH}$ and DIC, and heating to $75^{\circ} \mathrm{C}$.

Unfortunately, this spectrum, as well as fluorescence studies, did not allow for conclusive assignment of the metal oxidation state and identity of the complex after activation.

\section{Kinetic studies of alcohol insertion into diisopropylcarbodiimide}

In a typical experiment, an NMR sample was prepared as described above in the presence of $100 \mu \mathrm{L}$ of mesitylene as internal standard ( $\delta=6.70$ and $2.15 \mathrm{ppm}$ ). Experiments for reagent-order determination were performed at variable concentrations of catalyst, alcohol, and carbodiimide, spanning one order of magnitude concentration differences while keeping the other reagent concentration constant. The sample tube was inserted into the probe of the Bruker Avance 300 spectrometer which had been previously set to the desired temperature $\left(T=75 \pm 0.1^{\circ} \mathrm{C}\right.$; checked with ethylene glycol temperature standard). Data were acquired every five minutes up to one and one-half hours. Reaction rates were determined by least-squares fit of product concentration versus time, and the collective rate data plotted to determine reagent orders (Figures S3-S5). 


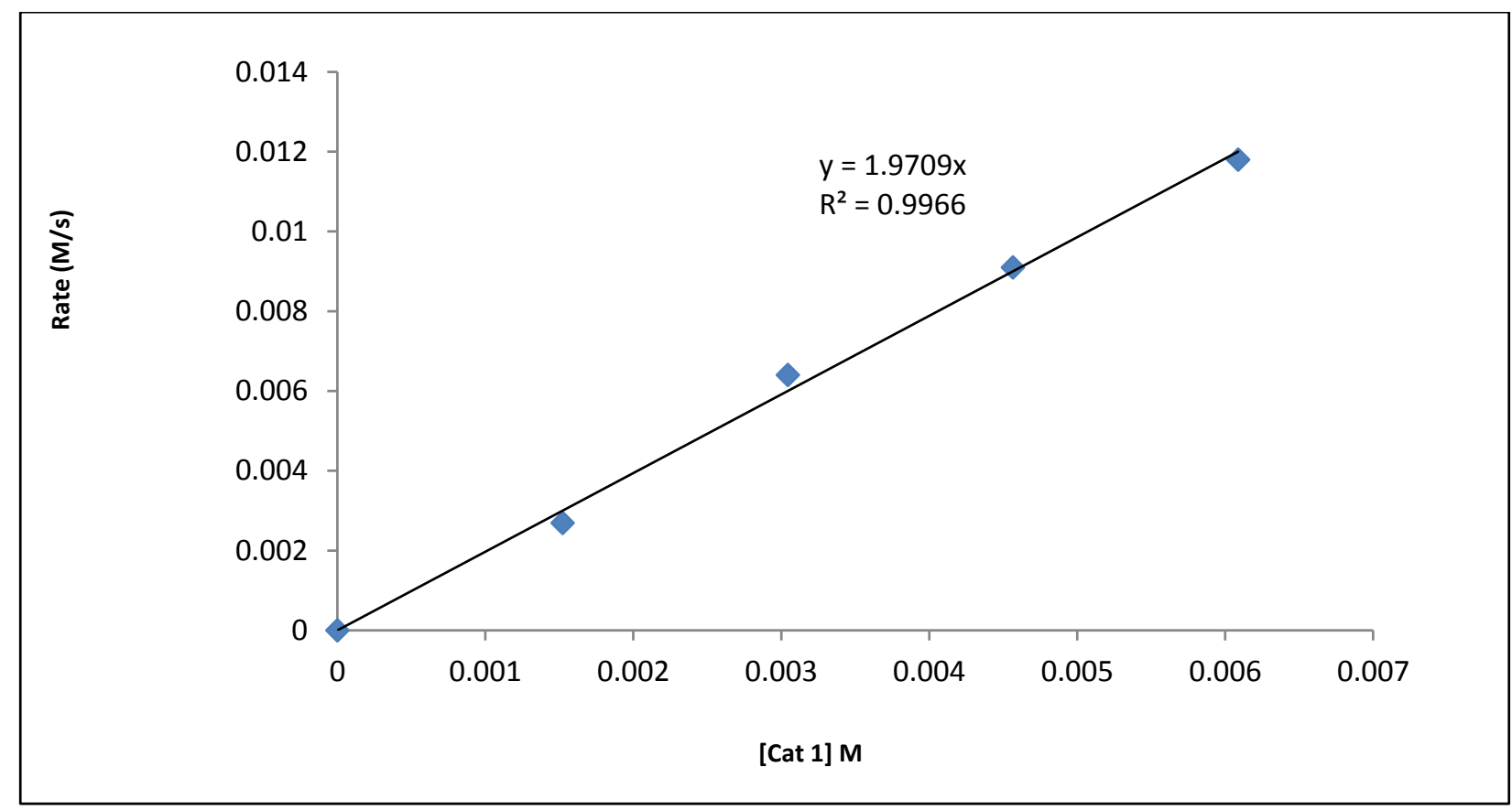

Figure S3: Plot of reaction rate as a function of precatalyst $\mathbf{1}$ concentration.

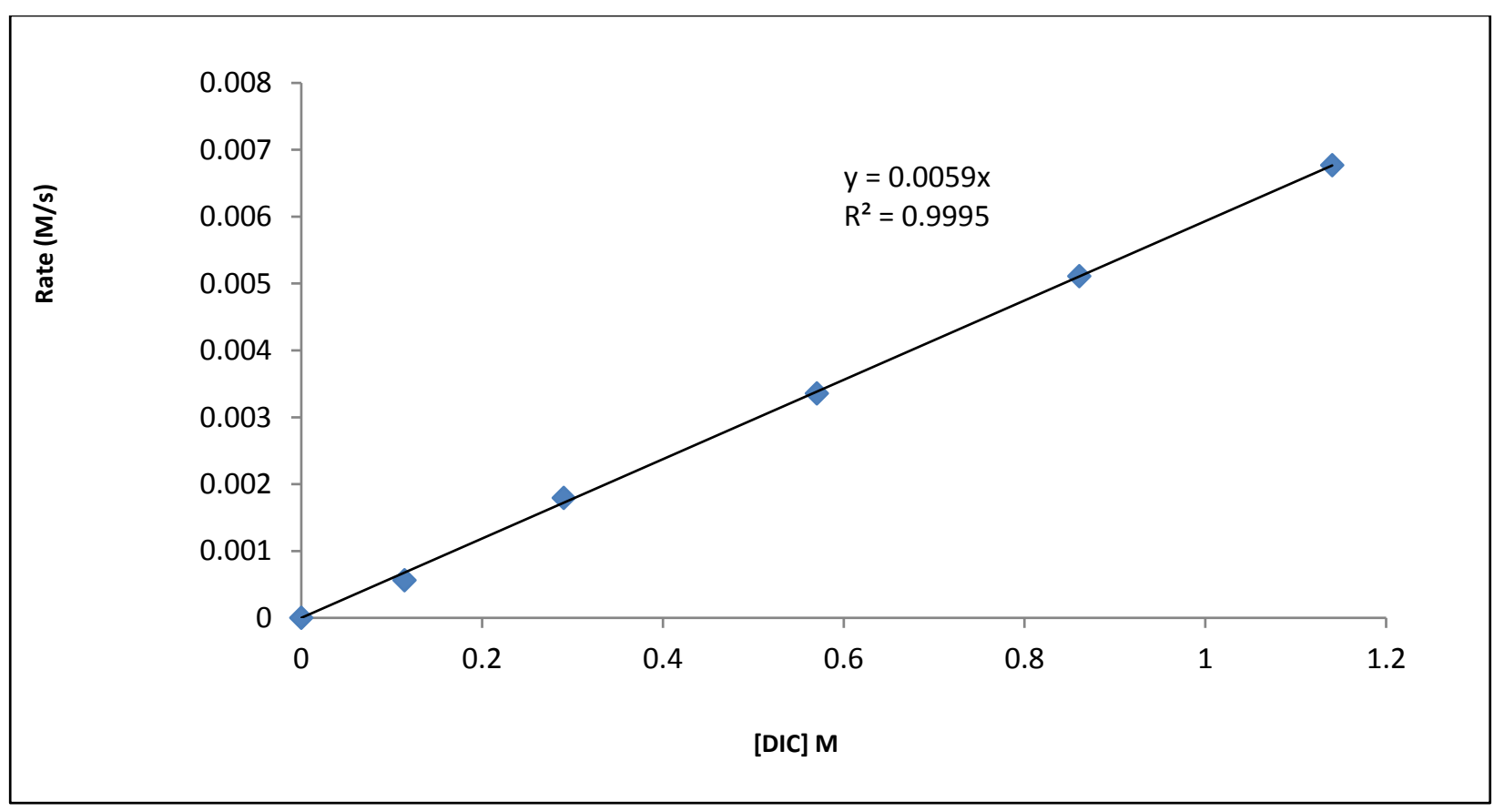

Figure S4: Plot of reaction rate as a function of diisopropylcarbodiimide concentration. 


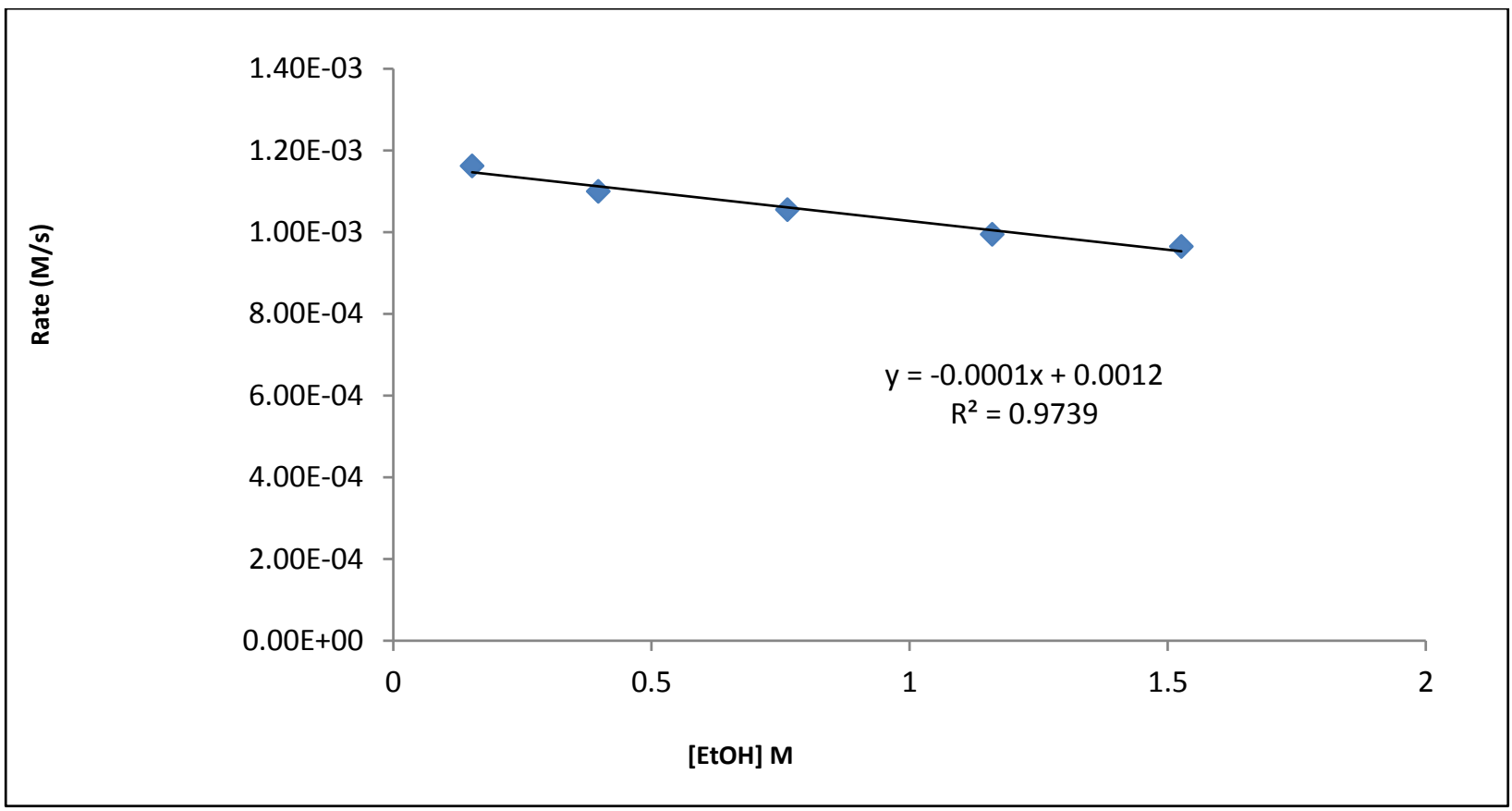

Figure S5: Plot of reaction rate as a function of alcohol concentration.

\section{Discussion of Kinetic Rate Law}

According to the kinetic and thermodynamic data obtained, the following mechanism is proposed: 


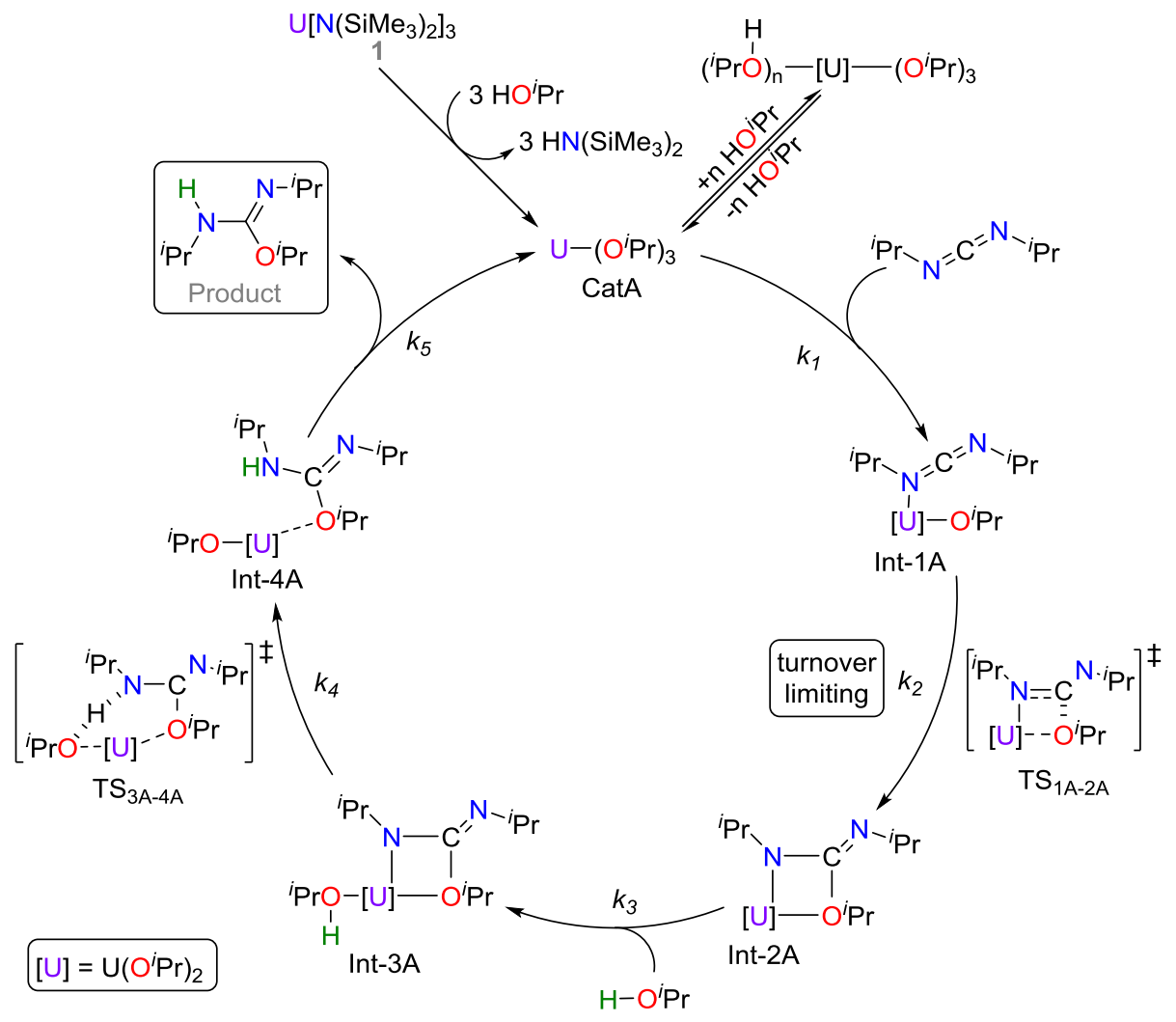

Since the first step of the cycle is turnover limiting $\left(k_{1}\right)$, and the remaining steps of the catalytic cycle are rapid $\left(k_{2}-k_{5}\right)$, we can assume a steady-state approximation for this reaction, giving the rate equation:

$$
\frac{\partial P}{\partial T}=k_{1}\left[\mathrm{Cat}_{A}\right][D I C]
$$

In describing the formation of the active catalyst, protonolysis of the amide starting material $\left(\mathrm{Cat}_{\mathrm{A}}\right)$ is rapid and irreversible, however we must consider the equilibrium of alcohol coordination/decoordination, which is described by the following equilibrium expression:

$$
K_{e q}=\frac{\left[\mathrm{satCat}_{A}\right]}{[\mathrm{ROH}]\left[\mathrm{Cat}_{A}\right]}
$$

In studying the relationship between the active catalysts $\left(\mathrm{Cat}_{\mathrm{A}}\right)$ and the alcohol-saturated complex (satCat ${ }_{A}$ ), we can see that the sum of these two species is the total amount of $\mathbf{1}$ used in the reaction: $\left[\mathrm{Cat}_{A}\right]+\left[\right.$ satCat $\left._{A}\right]=[\mathbf{1}]$. By substituting Cat $_{A}$ from the equilibrium expression into the rate equation, we see that the kinetic rate law for this reaction is:

$$
\frac{\partial P}{\partial T}=\frac{k_{1}[D I C][\mathbf{1}]}{\left(1+K_{e q}[R O H]\right)}
$$

From this equation, we can see that if $[\mathrm{ROH}]$ is large, we see inverse-first order behavior, however when $[\mathrm{ROH}]$ is small, we see nearly zero-order behavior in alcohol. 


\section{Thermodynamic studies of aniline insertion into diisopropylcarbodiimide}

Activation parameters $\left(\Delta H^{\ddagger}\right.$ and $\left.\Delta S^{\ddagger}\right)$ were calculated from acquired kinetic data using Eyring plots. In a typical experiment, an NMR sample was prepared as described above in the presence of $100 \mu \mathrm{L}$ of mesitylene as internal standard ( $\delta=6.70$ and $2.15 \mathrm{ppm}$ ). The sample tube was inserted into the probe of the Bruker Avance 300 spectrometer which had been previously set to the desired temperature over the range of $35-75^{\circ} \mathrm{C}\left(\mathrm{T} \pm 0.1{ }^{\circ} \mathrm{C}\right.$; checked with ethylene glycol temperature standard). Data were acquired five minutes up to one and one-half hours. Reaction rates were determined by leastsquares fit of product concentration versus time. Eyring plots were generated in the usual manner (Figures $\mathrm{S} 6-\mathrm{S} 8$ ), and the $\Delta H^{\ddagger}$ and $\Delta S^{\ddagger}$ values for activation calculated from the slope and intercept of the least-squares fit, respectively.

Eyring plots of actinide-mediated carbodiimide insertion into alcohols

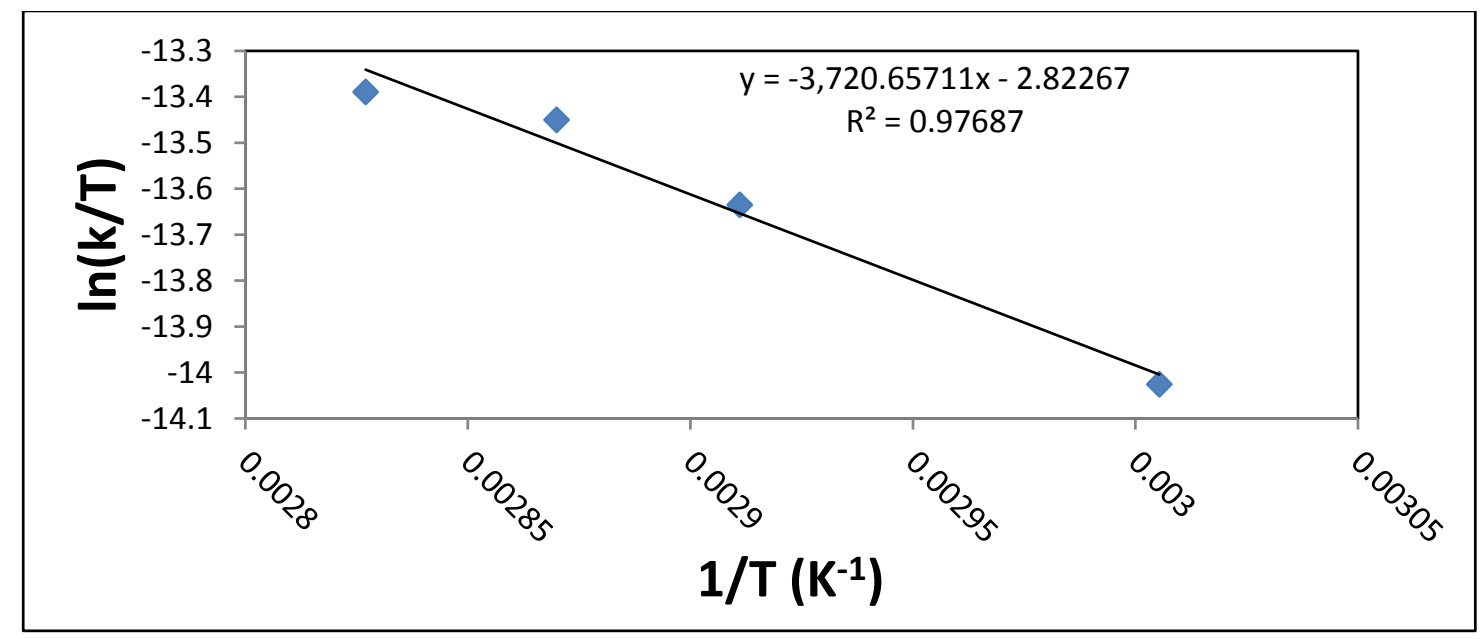

Figure S6: Eyring plot of ethanol addition to diisopropylcarbodiimide mediated by complex 1.

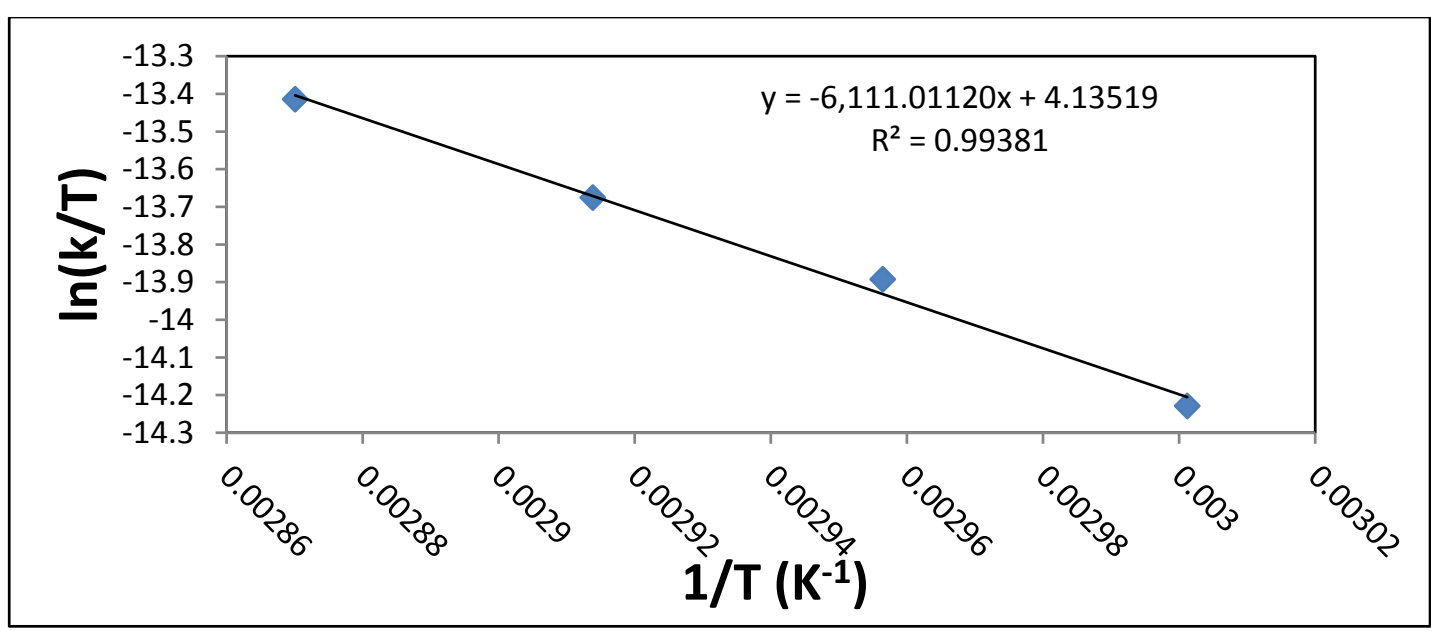

Figure S7: Eyring plot of tert-butanol addition to diisopropylcarbodiimide mediated by complex 2. 


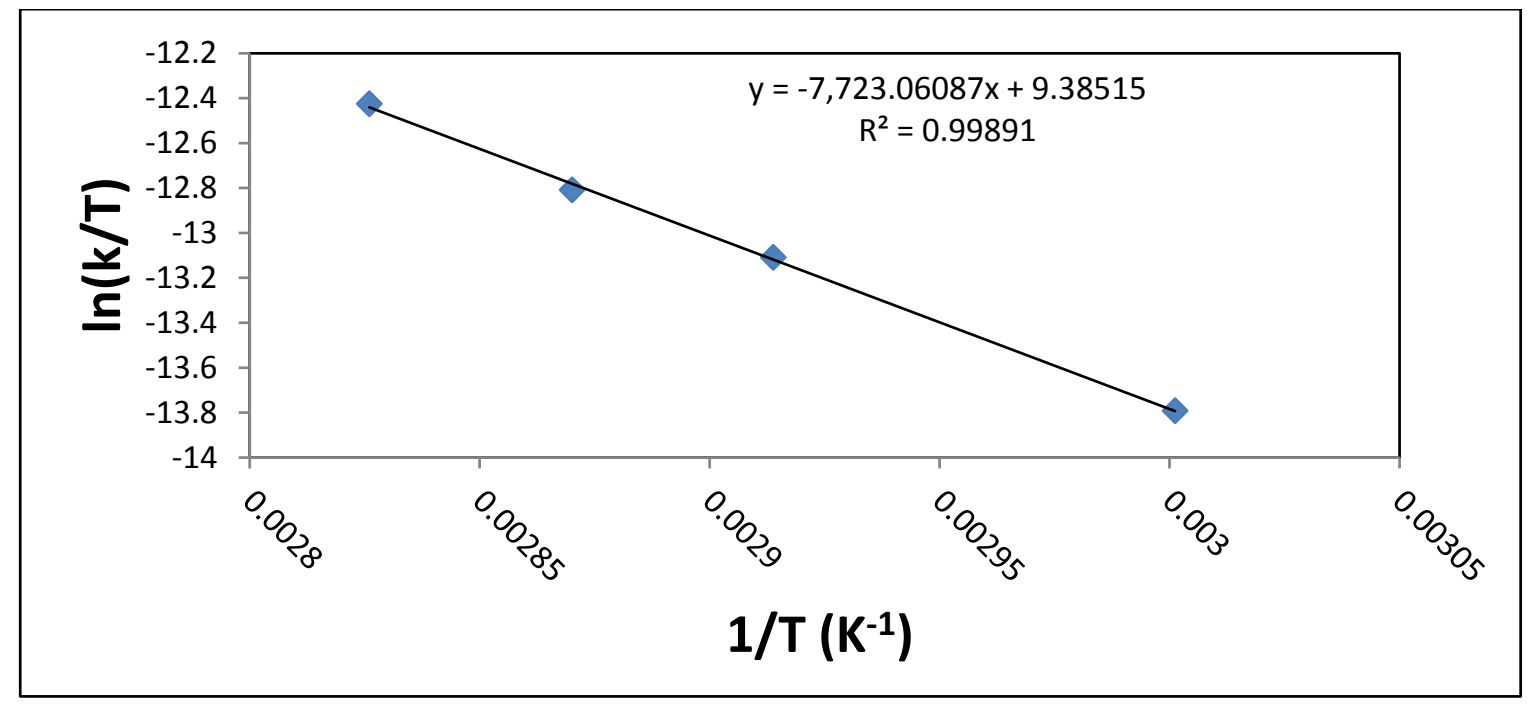

Figure S8: Eyring plot of ethanol addition to diisopropylcarbodiimide mediated by complex 3.

\section{Computational Details}

All the structures reported in this study were fully optimised with the Becke's 3-parameter hybrid functional combined with the non-local correlation functional provided by Perdew/Wang (denoted as B3PW91). ${ }^{4}$ The choice of the basis set for Cat $_{\mathrm{A}}$, which preserves a fixed oxidation state (III) throughout the catalytic process, is the quasi-relativistic energy-consistent 5 -in-core RECP (Relativistic Effective Core Potential), augmented by a $f$ polarization function, in combination with its adapted basis set. ${ }^{5}$ The analogous $5 f$-in-core RECPs (augmented by a $f$ polarization function) are used for the fixed oxidation states (IV) of the corresponding $U$ and Th catalytic systems, Cat $_{\mathrm{B}}$ and Cat $_{\mathrm{c}}$ respectively. ${ }^{6}$ In addition, for the silicon atoms the quasi-relativistic energy-adjusted ab-initio pseudopotential is used, along with its corresponding energy-optimized valence basis set, ${ }^{7}$ augmented by a $d$ polarization function. ${ }^{8}$ For the rest of the atoms the $6-31 \mathrm{G}(\mathrm{d}, \mathrm{p})$ basis set is used. ${ }^{9}$ In all computations no constrains are imposed on the geometry. All stationary points have been identified for minimum (number of imaginary frequencies Nimag=0) or transition states (Nimag=1). Intrinsic Reaction Paths (IRPs) are traced from the various transition structures to verify the reactant to product linkage. ${ }^{10}$ Enthalpy energies are obtained at $\mathrm{T}=298.15 \mathrm{~K}$ within the harmonic approximation. GAUSSIAN09 program suite is used in all calculations. ${ }^{11}$ Finally, for the 3D representation of the structures the Cylview graphical program is used. ${ }^{12}$

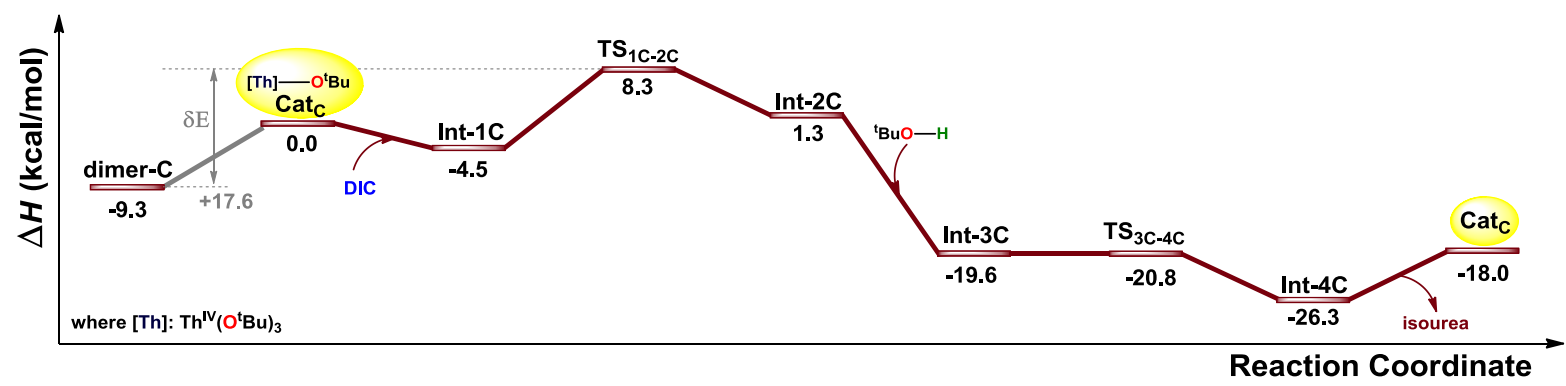

Figure S9: Plausible reaction profile of ${ }^{t} \mathrm{BuOH}$ addition to DIC mediated by complex 3. 


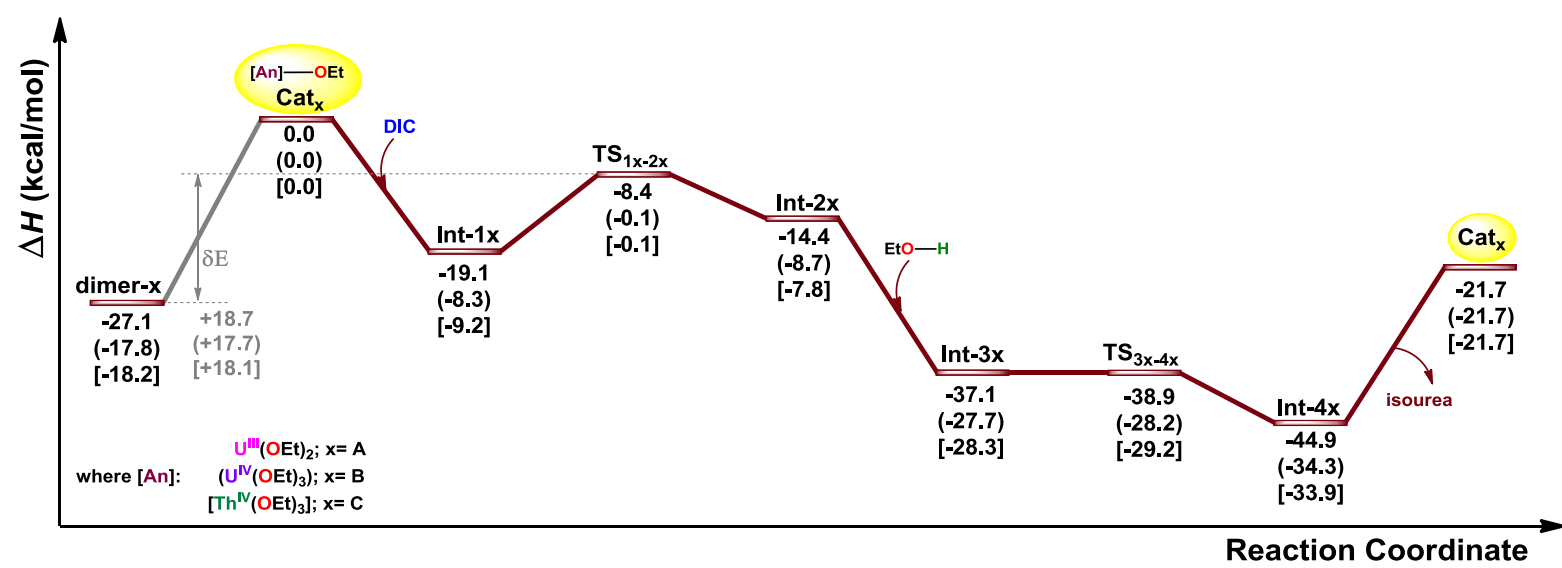

Figure S10: Plausible reaction profile of EtOH addition to DIC mediated by complex 1, 2 and $\mathbf{3}$.

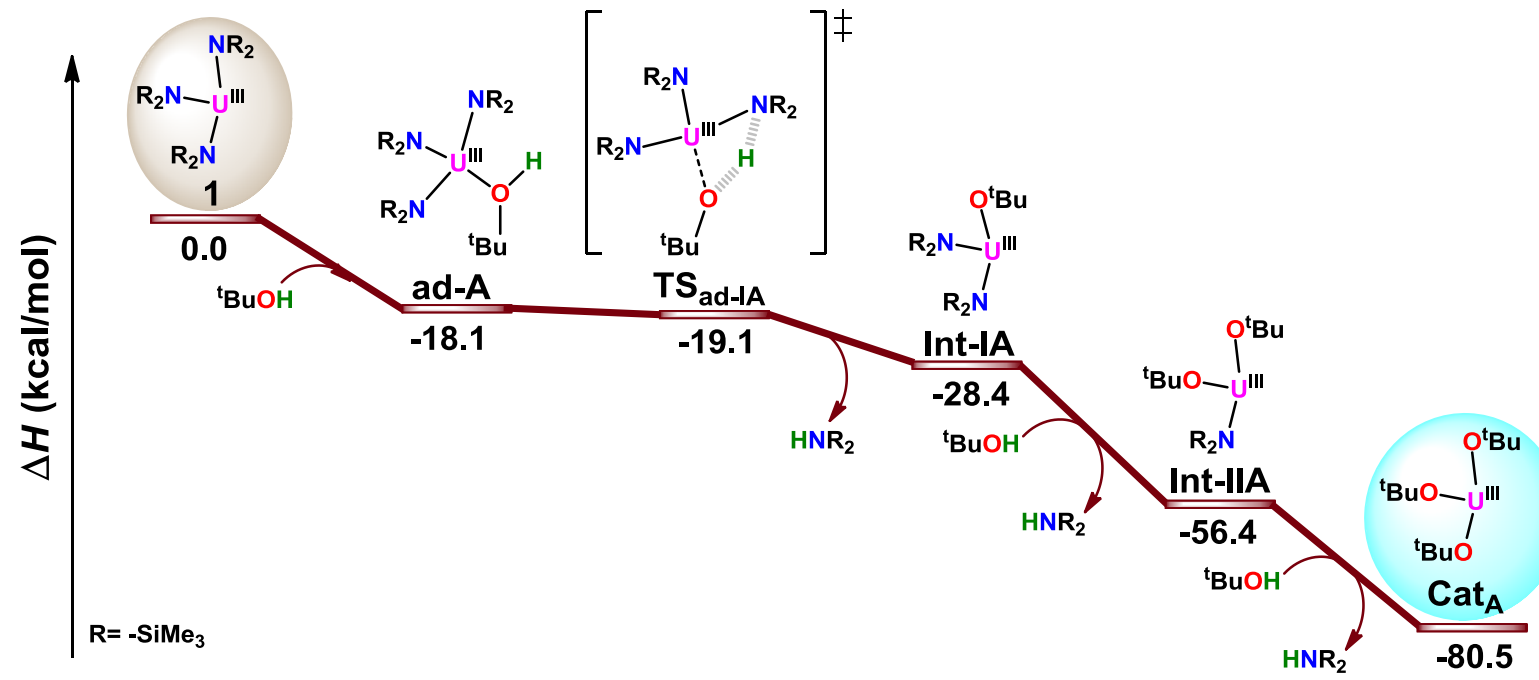

Figure S11: Enthalpy energy profile for the formation model catalyst Cat $_{A}$ by consecutive additions of ${ }^{t} \mathrm{BuOH}$ at complex 1. 


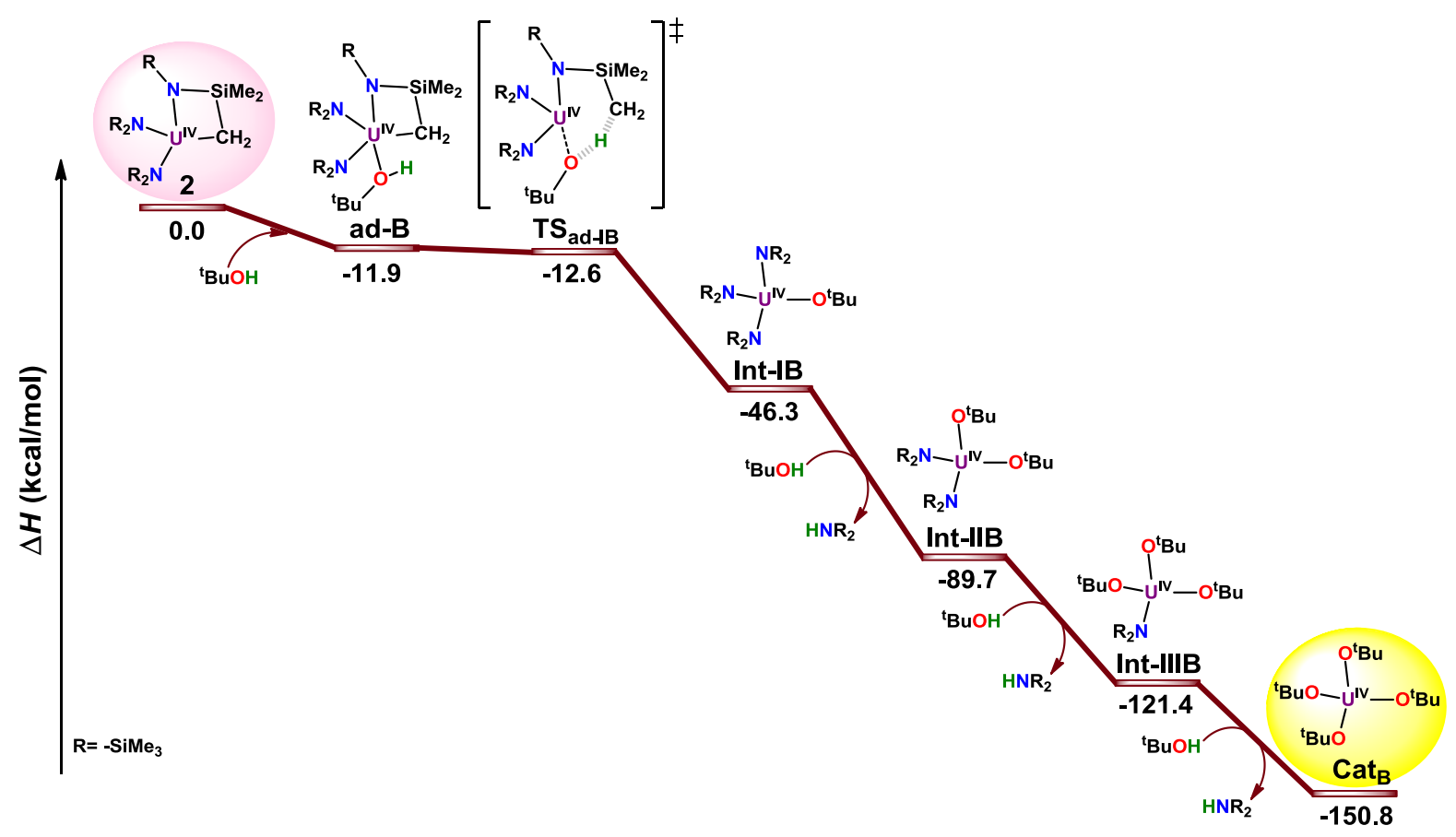

Figure S12: Enthalpy energy profile for the formation model catalyst Cat $_{\mathrm{B}}$ by consecutive additions of ${ }^{t} \mathrm{BuOH}$ at complex $\mathbf{2}$.

\section{Enthalpy energies in a.u. along with Cartesian coordinates of the optimized structures.}

$\begin{array}{lrrr}\text { pre-Cat } & & & \\ H=-937.364609 a u & & \\ \mathrm{U} & 0.12983200 & 1.00653100 & 0.09761600 \\ \mathrm{Si} & 0.53143100 & 3.86079500 & 1.87807700 \\ \mathrm{Si} & 2.09898100 & 1.55733100 & 3.20189400 \\ \mathrm{Si} & -3.24161000 & 0.51977500 & 1.56756100 \\ \mathrm{Si} & -2.83489300 & 0.64385600 & -1.49381800 \\ \mathrm{Si} & 0.98374700 & -2.54450500 & -0.52665300 \\ \mathrm{Si} & 2.87322700 & -0.27320700 & -1.43423100 \\ \mathrm{~N} & 1.01991900 & 2.19372400 & 1.97643600 \\ \mathrm{~N} & -2.23465900 & 0.64203300 & 0.13852800 \\ \mathrm{~N} & 1.43462200 & -0.85582200 & -0.64874200 \\ \mathrm{C} & 3.08037100 & -0.86214400 & -3.23678400 \\ \mathrm{H} & 2.19061100 & -0.61961400 & -3.83245600 \\ \mathrm{H} & 3.95425400 & -0.38329500 & -3.69993700 \\ \mathrm{H} & 3.22893700 & -1.94974100 & -3.27308500 \\ \mathrm{C} & 4.49847800 & -0.62188900 & -0.50292800 \\ \mathrm{H} & 4.68877400 & -1.70088900 & -0.44470400 \\ \mathrm{H} & 5.34734100 & -0.14981700 & -1.01747100 \\ \mathrm{H} & 4.44997500 & -0.22890700 & 0.52045000 \\ \mathrm{C} & 2.72742900 & 1.65884700 & -1.51798400 \\ \mathrm{H} & 1.87881800 & 2.01714500 & -2.12874600 \\ \mathrm{H} & 2.72666600 & 2.14815600 & -0.52913400 \\ \mathrm{H} & 3.62840500 & 2.02516100 & -2.03092100 \\ \mathrm{C} & -1.35635000 & 1.14487400 & -2.64774200 \\ \mathrm{H} & -0.53462400 & 0.40919700 & -2.67647600 \\ \mathrm{H} & -1.75744000 & 1.17872400 & -3.67118300 \\ \mathrm{H} & -0.96235700 & 2.15952200 & -2.45691700 \\ \mathrm{C} & -4.20898000 & 1.92397100 & -1.82740800 \\ \mathrm{H} & -4.46572300 & 1.94323200 & -2.89567200 \\ \mathrm{H} & -5.11609100 & 1.66909000 & -1.26337200 \\ \mathrm{H} & -3.89065300 & 2.92998300 & -1.52442700\end{array}$

$-3.42709200-1.04920500-2.13515400$ $-4.30061700-1.39492600-1.56823100$

$-3.70748400-0.98625500-3.19602600$

$-2.63377000-1.79963100-2.02854200$

$\begin{array}{lll}-2.11717300 & 0.17024600 & 3.07193600\end{array}$

$\begin{array}{lll}-1.65287200 & -0.82240900 & 2.99334400\end{array}$

$\begin{array}{lll}-1.32322900 & 0.92487300 & 3.17008600\end{array}$

$\begin{array}{lll}-2.71362500 & 0.19026800 & 3.99480400\end{array}$

$\begin{array}{lll}-4.52218400 & -0.89242300 & 1.45237400\end{array}$

$\begin{array}{lll}-5.07175400 & -0.98005600 & 2.40004800\end{array}$

$\begin{array}{lll}-5.25195200 & -0.70429500 & 0.65311600\end{array}$

$\begin{array}{lll}-4.02687800 & -1.85083200 & 1.24931600\end{array}$

$\begin{array}{lll}-4.19651000 & 2.13338200 & 1.93351400\end{array}$

$\begin{array}{lll}-4.80371700 & 2.01801000 & 2.84244800\end{array}$

$\begin{array}{lll}-3.50068600 & 2.96800300 & 2.09050800\end{array}$

$\begin{array}{lll}-4.86514400 & 2.39635000 & 1.10400000\end{array}$

$\begin{array}{lll}-0.36835300 & 4.09094300 & 0.17508200\end{array}$

$\begin{array}{lll}-0.65643700 & 5.15021600 & 0.10970200\end{array}$

$\begin{array}{llll}0.28263100 & 3.91152500 & -0.69980100\end{array}$

$\begin{array}{lll}-1.30852900 & 3.52094100 & 0.08161300\end{array}$

$\begin{array}{lll}-0.73095600 & 4.40863800 & 3.19614000\end{array}$

$\begin{array}{llll}-0.29521400 & 4.34770100 & 4.20127700\end{array}$

$\begin{array}{lll}-1.04764900 & 5.44636500 & 3.01999500\end{array}$

$\begin{array}{lll}-1.61945500 & 3.76522000 & 3.17054000\end{array}$

$\begin{array}{lll}1.97305500 & 5.10960400 & 1.87396000\end{array}$

$\begin{array}{llll}2.69577700 & 4.87154100 & 1.08251500\end{array}$

$\begin{array}{lll}1.59573800 & 6.12856900 & 1.70999700\end{array}$

$\begin{array}{lll}2.50260000 & 5.09319600 & 2.83593200\end{array}$

$\begin{array}{lll}1.97581600 & -0.34804100 & 3.18791400\end{array}$

$\begin{array}{lll}0.97204500 & -0.67689500 & 3.49046200\end{array}$

$\begin{array}{lll}2.19773400 & -0.76052100 & 2.19321400\end{array}$

$\begin{array}{llll}2.69885400 & -0.77449300 & 3.89713300\end{array}$ 
$\begin{array}{llll}C & 1.65991500 & 2.15707800 & 4.96109500\end{array}$

H $\quad 1.79583500 \quad 3.24262700 \quad 5.06031700$

$\begin{array}{llll}\text { H } & 0.61691300 & 1.91510500 & 5.20300900\end{array}$

H $\quad 2.31016500 \quad 1.66556300 \quad 5.69820800$

C $\quad 3.92465000 \quad 2.01915900 \quad 2.87898200$

$\begin{array}{llll}\mathrm{H} & 4.57262700 & 1.57242500 & 3.64633800\end{array}$

$\begin{array}{llll}\mathrm{H} & 4.25190900 & 1.65071700 & 1.89795800\end{array}$

H $\quad 4.06624200 \quad 3.10737800 \quad 2.90105900$

C $\quad 0.30296100 \quad-3.24462600 \quad-2.16817400$

H $\quad-0.58974400 \quad-2.69122800-2.48767500$

H $\quad 1.05139000 \quad-3.17777900-2.96795300$

$\begin{array}{llll}\mathrm{H} & 0.02475800 & -4.30071400 & -2.04395500\end{array}$

$\begin{array}{lllll}\text { C } & 2.43218900 & -3.66544100 & 0.01371200\end{array}$

H $\quad 3.22630600 \quad-3.68494200 \quad-0.74504100$

$\begin{array}{llll}\mathrm{H} & 2.86607800 & -3.31453600 & 0.95894300\end{array}$

$\begin{array}{lllll}H & 2.07485300 & -4.69475000 & 0.15722000\end{array}$

$\begin{array}{llll}\text { C } & -0.39975100 & -2.70711800 & 0.77992800\end{array}$

$\begin{array}{llll}\mathrm{H} & -0.76913600 & -3.74189500 & 0.80495400\end{array}$

$\begin{array}{llll}\mathrm{H} & -0.02370400 & -2.45950100 & 1.78217200\end{array}$

$\begin{array}{llll}H & -1.25286500 & -2.05217800 & 0.55044900\end{array}$

\section{dimer-A (EtOH)}

$H=-989.913284 a u$

$\begin{array}{llll}0 & -0.70023800 & 0.78792900 & 2.79293000\end{array}$

$\begin{array}{llll}\text { U } & -1.02384900 & 0.51717600 & 0.39492400\end{array}$

$\begin{array}{lllll}0 & -2.74188800 & -0.74707000 & -0.16345600\end{array}$

$\begin{array}{lllll}0 & 0.87477500 & 0.07573800 & -0.63268400\end{array}$

C $\quad-3.73079300-1.64088000-0.56409200$

$\begin{array}{lllll}\text { C } & -3.35405600 & -3.08860400 & -0.26664400\end{array}$

H $\quad-2.43124600-3.36067800 \quad-0.79069200$

H $\quad-3.18966500 \quad-3.22859000 \quad 0.80749900$

H $\quad-4.14419100 \quad-3.77921000 \quad-0.58390200$

C $\quad 2.00679200 \quad-0.25507800 \quad-1.37188300$

$\begin{array}{lllll}C & 2.06818400 & 0.50168600 & -2.69470200\end{array}$

$\begin{array}{lllll}\mathrm{H} & 1.19527100 & 0.26541400 & -3.31334600\end{array}$

H $\quad 2.07736600 \quad 1.58257300 \quad-2.51654100$

H $\quad 2.96913300 \quad 0.24056500 \quad-3.26211700$

$\begin{array}{lllll}\text { O } & -1.46439700 & 2.88493600 & 0.78728500\end{array}$

$\begin{array}{llll}\text { U } & -1.14387500 & 3.15531600 & 3.18548500\end{array}$

$\begin{array}{lllll}0 & -3.04642500 & 3.59010100 & 4.20827900\end{array}$

$\begin{array}{lllll}0 & 0.56616700 & 4.42887800 & 3.74816400\end{array}$

$\begin{array}{lllll}\text { C } & -4.18160500 & 3.91829600 & 4.94380400\end{array}$

$\begin{array}{llll}\text { C } & -4.24675400 & 3.15941400 & 6.26523600\end{array}$

$\begin{array}{llll}\mathrm{H} & -3.37645600 & 3.39599500 & 6.88742800\end{array}$

$\begin{array}{lllll}H & & -5.15015300 & 3.41839900 & 6.82972600\end{array}$

$\begin{array}{llll}\text { H } & -4.25373100 & 2.07880200 & 6.08535900\end{array}$

$\begin{array}{llll}\text { C } & 1.54978700 & 5.33059700 & 4.14402200\end{array}$

$\begin{array}{lllll}\text { C } & 1.29787300 & 6.73219800 & 3.59787600\end{array}$

H $\quad 1.273042006 .71728600 \quad 2.50268400$

$\begin{array}{llll}\mathrm{H} & 0.33609700 & 7.11630900 & 3.95556500\end{array}$

$\begin{array}{llll}\text { H } & 2.08291400 & 7.42972300 & 3.91256300\end{array}$

C $\quad-1.85659800 \quad 3.93953400 \quad-0.07526000$

$\begin{array}{llll}\text { C } & -3.28657200 & 3.77500600 & -0.56961500\end{array}$

H $\quad-3.40445100 \quad 2.84540400 \quad-1.13888600$

H $\quad-3.98583000 \quad 3.74377600 \quad 0.27202400$

H $\quad-3.57204200 \quad 4.60605100 \quad-1.22376400$

$\begin{array}{llll}\text { C } & -0.30824000 & -0.26852400 & 3.65311900\end{array}$

$\begin{array}{llll}\text { C } & 1.11757200 & -0.09898900 & 4.15769700\end{array}$

$\begin{array}{llll}\mathrm{H} & 1.82216900 & -0.06029700 & 3.32085100\end{array}$

$\begin{array}{llll}\mathrm{H} & 1.22684700 & 0.82850100 & 4.73216300\end{array}$

H $\quad 1.40303700-0.93152900 \quad 4.80993500$

$\begin{array}{llll}\mathrm{H} & -1.00323900 & -0.33764900 & 4.50433700\end{array}$

H $\quad-0.38871900 \quad-1.23589000 \quad 3.12685800$
$\begin{array}{lllll}\mathrm{H} & -1.76724400 & 4.90879700 & 0.44585500\end{array}$

H $\quad-1.16689300 \quad 4.00100100 \quad-0.93139400$

$\begin{array}{llll}\mathrm{H} & -5.09131100 & 3.69570300 & 4.35971100\end{array}$

H $\quad-4.20781000 \quad 5.00173000 \quad 5.15285600$

H $\quad 1.60528100 \quad 5.38238600 \quad 5.24522900$

$\begin{array}{llll}\mathrm{H} & 2.54178800 & 4.98719300 & 3.80288900\end{array}$

H $\quad 2.91895900-0.03288000 \quad-0.79147000$

$\mathrm{H} \quad 2.03079700-1.33886200 \quad-1.57936900$

H $\quad-4.68353600 \quad-1.40805400 \quad-0.05774200$

H $\quad-3.92627400 \quad-1.53913900-1.64571200$

\section{$\mathrm{Cat}_{\mathrm{A}}(\mathrm{EtOH})$}

$H=-494.913388 a u$

$\begin{array}{llll}\text { U } & 0.98692500 & 2.51761500 & -0.53975400\end{array}$

C $\quad 4.75813000 \quad 0.47502000 \quad-1.39226900$

H $\quad 5.16913900 \quad 1.42973100 \quad-1.73812200$

H $\quad 4.74170300 \quad 0.48965200 \quad-0.29735500$

H $\quad 5.43340100-0.32571600 \quad-1.71589700$

$\begin{array}{lllll}\text { C } & -2.12411500 & 0.20020400 & -2.48974000\end{array}$

H $\quad-1.22533400-0.40710800-2.64044600$

H $\quad-3.00087900-0.44785700 \quad-2.60501200$

H $\quad-2.15453300 \quad 0.96078700 \quad-3.27744700$

$\begin{array}{llll}\text { C } & 0.29860900 & 1.32101500 & 3.50259900\end{array}$

$\mathrm{H} \quad \begin{array}{llll}\mathrm{H} & -0.54894000 & 2.01420600 & 3.48330600\end{array}$

$\begin{array}{llll}\mathrm{H} & 0.02578200 & 0.43775900 & 2.91506600\end{array}$

$\begin{array}{llll}\mathrm{H} & 0.46212200 & 1.00533300 & 4.53971000\end{array}$

$\begin{array}{lllll}\text { O } & 2.48192100 & 1.28270400 & -1.54923400\end{array}$

$\begin{array}{lllll}0 & 1.35775500 & 2.41508600 & 1.61424000\end{array}$

$\begin{array}{lllll}0 & -0.99952400 & 1.69610600 & -0.95169400\end{array}$

C $\quad \begin{array}{lllll}\text { C } & -2.10046000 & 0.85802700 & -1.11436100\end{array}$

$\begin{array}{llll}C & 3.35182000 & 0.26876800 & -1.94429500\end{array}$

$\begin{array}{llll}\text { C } & 1.54412200 & 1.98490600 & 2.92606300\end{array}$

$\begin{array}{lllll}\mathrm{H} & -2.10684000 & 0.07369400 & -0.33860000\end{array}$

H $\quad-3.03191600 \quad 1.43290800 \quad-0.97433700$

H $\quad 3.40019700 \quad 0.22253100 \quad-3.04573400$

$\mathrm{H} \quad 2.97549300 \quad-0.71391400-1.61337600$

H $\quad \begin{array}{llll}\text { H } & 1.81969600 & 2.84345000 & 3.56229300\end{array}$

$\begin{array}{llll}H & 2.38986400 & 1.27843200 & 2.98351000\end{array}$

\section{Int-1A (EtOH)}

$H=-879.261157 \mathrm{au}$

$\begin{array}{llll}\mathrm{N} & -0.93848800 & 2.23361100 & 0.51992600\end{array}$

$\begin{array}{llll}\text { C } & -0.91041600 & 2.52780300 & 1.73128500\end{array}$

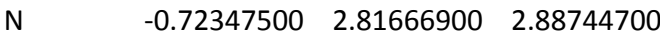

$\begin{array}{lllll}\mathrm{O} & 0.87305200 & -1.00591200 & 1.80774600\end{array}$

$\begin{array}{llll}\text { C } & 1.44443300 & -1.83855700 & 2.76151400\end{array}$

$\begin{array}{lllll}\text { C } & 2.44662800 & -2.82009500 & 2.15961000\end{array}$

H $\quad 3.25588000 \quad-2.28011400 \quad 1.65590300$

$\begin{array}{llll}\mathrm{H} & 2.89022900 & -3.46301200 & 2.92942200\end{array}$

$\mathrm{H} \quad 1.95586200-3.46382000 \quad 1.42085000$

U $\quad 0.25533100-0.12712000-0.14855900$

O $\quad-1.42626300-1.23464400-1.08671700$

$\begin{array}{lllll}0 & & 1.90264400 & 0.20868500 & -1.59157700\end{array}$

C $\quad-2.25498900-2.18223600 \quad-1.67333900$

C $\quad-1.97697500-3.59438000-1.16589300$

$\mathrm{H} \quad-0.94551400 \quad-3.88699800 \quad-1.39245100$

$\mathrm{H} \quad-2.11116300-3.64318900-0.07983900$

H $\quad-2.64862000 \quad-4.32674700 \quad-1.62986000$

$\begin{array}{lllll}\text { C } & 2.93305500 & 0.16533400 & -2.52096800\end{array}$

$\begin{array}{llll}\text { C } & 2.47349100 & -0.37063000 & -3.87428800\end{array}$

H $\quad 2.07982400 \quad-1.38752900 \quad-3.76977000$

H $\quad \begin{array}{llll}1.67495600 & 0.25767900 & -4.28369900\end{array}$ 


$\begin{array}{lrrr}H & 3.29839900 & -0.39348800 & -4.59673100 \\ \mathrm{C} & -1.28304500 & 2.31187300 & 4.14648900 \\ \mathrm{C} & -1.61826200 & 3.49643400 & 5.04527800 \\ \mathrm{C} & -0.26103200 & 1.36576600 & 4.77306500 \\ \mathrm{H} & -2.20148800 & 1.75234400 & 3.92518500 \\ \mathrm{H} & -2.35156600 & 4.15744800 & 4.57460200 \\ \mathrm{H} & -2.03589000 & 3.13807600 & 5.99070900 \\ \mathrm{H} & -0.71795200 & 4.07902600 & 5.26229600 \\ \mathrm{H} & -0.01583100 & 0.54673700 & 4.09134400 \\ \mathrm{H} & 0.66050400 & 1.90460900 & 5.01348700 \\ \mathrm{H} & -0.66774200 & 0.94457000 & 5.69761500 \\ \mathrm{C} & -1.79079900 & 2.96776500 & -0.46065300 \\ \mathrm{C} & -1.64252000 & 2.29616300 & -1.81963500 \\ \mathrm{C} & -1.40884900 & 4.44438400 & -0.49819900 \\ \mathrm{H} & -2.83331200 & 2.86982000 & -0.13058900 \\ \mathrm{H} & -1.93254700 & 1.23887000 & -1.78404300 \\ \mathrm{H} & -2.28464000 & 2.78962200 & -2.55484300 \\ \mathrm{H} & -0.60924400 & 2.37188300 & -2.18003000 \\ \mathrm{H} & -1.52521400 & 4.91505700 & 0.48319700 \\ \mathrm{H} & -0.36826400 & 4.56393300 & -0.81601000 \\ \mathrm{H} & -2.04795100 & 4.97916500 & -1.20754200 \\ \mathrm{H} & 1.96167500 & -1.24160100 & 3.53534900 \\ \mathrm{H} & 0.66490300 & -2.41371500 & 3.29420200 \\ \mathrm{H} & 3.36033800 & 1.17413700 & -2.66627700 \\ \mathrm{H} & 3.76486100 & -0.46299100 & -2.15453400 \\ \mathrm{H} & -2.14344500 & -2.17236700 & -2.77274800 \\ \mathrm{H} & -3.31486200 & -1.93930500 & -1.47462500\end{array}$

$\begin{array}{llll}\text { TS } 1 \text { A-2A } & \text { (EtOH) } & & \\ H=-879.244091 a u & & \\ \mathrm{~N} & -1.09944900 & 2.52925000 & 0.94635400 \\ \mathrm{C} & -0.94830700 & 2.38786700 & 2.21671700 \\ \mathrm{~N} & -0.98047000 & 2.89729700 & 3.33440100 \\ \mathrm{O} & -0.27261500 & 0.54405700 & 2.25152600 \\ \mathrm{C} & 0.73582300 & 0.31397400 & 3.20225300 \\ \mathrm{C} & 1.94723200 & -0.39384200 & 2.60350000 \\ \mathrm{H} & 2.39761700 & 0.19679300 & 1.79701100 \\ \mathrm{H} & 2.71953900 & -0.56337600 & 3.36205800 \\ \mathrm{H} & 1.66905400 & -1.37659500 & 2.20050600 \\ \mathrm{U} & -0.39281700 & 0.33440300 & -0.12166000 \\ \mathrm{O} & -2.09019300 & -0.77437800 & -1.00872000 \\ \mathrm{O} & 1.49590400 & 0.44175200 & -1.26547600 \\ \mathrm{C} & -3.12188100 & -1.53938000 & -1.54198500 \\ \mathrm{C} & -3.63830100 & -2.57905700 & -0.55191400 \\ \mathrm{H} & -2.83538000 & -3.26699100 & -0.26429300 \\ \mathrm{H} & -4.00739200 & -2.09137900 & 0.35684900 \\ \mathrm{H} & -4.45582300 & -3.16935200 & -0.98246100 \\ \mathrm{C} & 2.53798300 & 0.41351700 & -2.18647900 \\ \mathrm{C} & 2.04023200 & 0.28471500 & -3.62289500 \\ \mathrm{H} & 1.46110400 & -0.63697400 & -3.74727600 \\ \mathrm{H} & 1.39088800 & 1.12895500 & -3.88064600 \\ \mathrm{H} & 2.87341600 & 0.26525700 & -4.33530000 \\ \mathrm{C} & -1.43692100 & 2.37945500 & 4.61191600 \\ \mathrm{C} & -2.94042500 & 2.62608500 & 4.76299200 \\ \mathrm{C} & -0.64463700 & 3.03683500 & 5.73890100 \\ \mathrm{H} & -1.26300700 & 1.29531000 & 4.63280500 \\ \mathrm{H} & -3.50065100 & 2.12535800 & 3.96770100 \\ \mathrm{H} & -3.29578600 & 2.24260700 & 5.72526600 \\ \mathrm{H} & -3.15996200 & 3.69769100 & 4.71715000 \\ \mathrm{H} & 0.42637400 & 2.84386100 & 5.62979500 \\ \mathrm{H} & -0.79555900 & 4.12120400 & 5.73001000 \\ & -0.96785900 & 2.65100300 & 6.71083400\end{array}$

$\begin{array}{rrr}-1.85628100 & 3.71824700 & 0.50052200 \\ -2.40010100 & 3.44622800 & -0.89787800 \\ -0.96627500 & 4.95959800 & 0.53686700 \\ -2.70191500 & 3.87402400 & 1.18566300 \\ -3.04821300 & 2.56315000 & -0.90949000 \\ -2.98427000 & 4.29673000 & -1.26225800 \\ -1.57777500 & 3.28994500 & -1.60911200 \\ -0.58995700 & 5.13184600 & 1.54891900 \\ -0.10974700 & 4.83550400 & -0.13451300 \\ -1.52577600 & 5.84694100 & 0.22158600 \\ 1.05662000 & 1.27192400 & 3.64118500 \\ 0.33714300 & -0.29329000 & 4.03196200 \\ 3.14896200 & 1.32902300 & -2.10029700 \\ 3.21968700 & -0.42728900 & -1.96869400 \\ -2.78924600 & -2.05475000 & -2.46021300 \\ -3.96221900 & -0.89063800 & -1.84547500\end{array}$

\section{Int-2A (EtOH)}

$H=-879.253606 a u$

$\begin{array}{llll}\mathrm{N} & -0.55910400 & 1.39450400 & 0.54148800\end{array}$

C $\quad \begin{array}{llll}-0.42521400 & 1.23501400 & 1.86908600\end{array}$

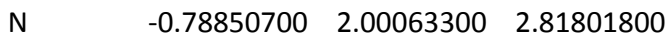

$\begin{array}{lllll}\text { O } & 0.20775200 & -0.07377800 & 2.08396800\end{array}$

$\begin{array}{llll}\text { C } & 1.50459800 & -0.02725500 & 2.71054900\end{array}$

$\begin{array}{llll}\text { C } & 2.34229100 & -1.18900000 & 2.20970100\end{array}$

H $\quad 2.58204400-1.09417700 \quad 1.14200400$

$\begin{array}{lllll}H & 3.29696600 & -1.22003200 & 2.74394000\end{array}$

$\begin{array}{lllll}\mathrm{H} & 1.83787900 & -2.14495500 & 2.39508700\end{array}$

U $\quad 0.02438200-0.77135700-0.43163100$

O $\quad-1.63556500-1.67154800-1.56165200$

O $\quad 1.95410100-0.63759800 \quad-1.47915500$

C $\quad-2.65989700 \quad-2.20803000 \quad-2.33924100$

C $\quad-3.06340300-3.60044300 \quad-1.86614600$

H $\quad-2.21117500 \quad-4.28689700 \quad-1.92029100$

H $\quad-3.40860900 \quad-3.56707700 \quad-0.82701900$

$\mathrm{H} \quad-3.87176900-4.01051900-2.48252400$

$\begin{array}{lllll}\text { C } & 2.97729300 & -0.49802100 & -2.41535700\end{array}$

C $\quad 2.47146000-0.61153000 \quad-3.84911300$

$\mathrm{H} \quad \begin{array}{llll}\mathrm{H} & 1.99720600 & -1.58522400 & -4.01349700\end{array}$

H $\quad \begin{array}{llll}1.72860800 & 0.16650400 & -4.05736900\end{array}$

H $\quad 3.28994700 \quad-0.50255900-4.57007100$

C $\quad \begin{array}{llll}0.81498200 & 1.61783200 & 4.21431100\end{array}$

$\begin{array}{llll}\text { C } & -2.25158500 & 1.76243100 & 4.72792000\end{array}$

$\begin{array}{llll}\text { C } & 0.13681100 & 2.50902500 & 5.01777400\end{array}$

$\begin{array}{lllll}\mathrm{H} & -0.52162000 & 0.56510700 & 4.36102900\end{array}$

$\begin{array}{llll}H & -2.92930900 & 1.11206700 & 4.16658800\end{array}$

$\begin{array}{llll}\mathrm{H} & -2.32000400 & 1.49956200 & 5.78969900\end{array}$

$\begin{array}{llll}\mathrm{H} & -2.59357400 & 2.79450200 & 4.60009200\end{array}$

$\mathrm{H} \quad 1.17121800 \quad 2.39565900 \quad 4.67732100$

$\begin{array}{llll}\mathrm{H} & -0.14063900 & 3.56022300 & 4.88843300\end{array}$

$\begin{array}{llll}\text { H } & 0.09804400 & 2.26890600 & 6.08610100\end{array}$

$\begin{array}{llll}\text { C } & -1.25236700 & 2.59669600 & 0.08834300\end{array}$

$\begin{array}{llll}\text { C } & -1.74909700 & 2.37022800 & -1.33787000\end{array}$

$\begin{array}{llll}\text { C } & -0.34494500 & 3.82679600 & 0.17300500\end{array}$

$\mathrm{H} \quad-2.11703400 \quad 2.77202900 \quad 0.74625200$

$\mathrm{H} \quad-2.42804300 \quad 1.51148200 \quad-1.39392200$

H $\quad-2.28682900 \quad 3.24634400 \quad-1.71409200$

H $\quad \begin{array}{llll}-0.90484800 & 2.19373300 & -2.01964200\end{array}$

$\begin{array}{llll}\mathrm{H} & -0.01313500 & 3.97415200 & 1.20357400\end{array}$

$\begin{array}{llll}H & 0.53494800 & 3.69745800 & -0.46786000\end{array}$

H $\quad-0.87634500 \quad 4.72922800 \quad-0.15018100$

$\begin{array}{llll}\mathrm{H} & 1.98156100 & 0.92985300 & 2.47148600\end{array}$

H $\quad \begin{array}{llll}\text { H } & 1.37982800 & -0.08339600 & 3.79671400\end{array}$ 
$\begin{array}{lllll}\mathrm{H} & 3.48085000 & 0.47506000 & -2.28615900\end{array}$

H $\quad 3.74976500-1.26770200 \quad-2.24607500$

$\mathrm{H} \quad-2.35082300-2.26431300 \quad-3.39673700$

$-3.54538200-1.55069400-2.31107000$

\section{Int-3A (EtOH)}

$H=-1034.178253 a u$

N $\quad \begin{array}{llll}-1.07642700 & 0.99036700 & 1.59885600\end{array}$

$\begin{array}{lllll}\text { C } & -0.53023600 & -0.02839800 & 2.29398500\end{array}$

$\begin{array}{llll}\mathrm{N} & -0.64716400 & -0.34507100 & 3.52262500\end{array}$

$\begin{array}{llll}0 & 0.21777400 & -0.84256400 & 1.33558300\end{array}$

$\begin{array}{llll}\text { C } & 1.63387900 & -0.99109500 & 1.57733600\end{array}$

$\begin{array}{llll}C & 2.40739300 & 0.28869700 & 1.32111000\end{array}$

$\begin{array}{llll}\mathrm{H} & 2.04080000 & 1.09996200 & 1.95737700\end{array}$

$\begin{array}{llll}\mathrm{H} & 3.46601500 & 0.13113000 & 1.55320600\end{array}$

$\begin{array}{llll}\mathrm{H} & 2.33343500 & 0.58039600 & 0.26812400\end{array}$

$\begin{array}{lllll}\text { U } & -0.70317400 & 0.21064800 & -0.84025300\end{array}$

$\begin{array}{lllll}0 & -2.51859900 & -0.57072200 & -1.81943700\end{array}$

O $\quad 1.19632200-0.17486100 \quad-1.92486900$

C $\quad-3.57774100 \quad-1.17631700 \quad-2.48935500$

C $\quad-3.92338800 \quad-2.54061500 \quad-1.90141500$

H $\quad-4.19632000-2.44392700-0.84510000$

$\mathrm{H} \quad-4.76445000 \quad-3.00178300 \quad-2.43250900$

H $\quad-3.06442000-3.21742000-1.96969600$

C $\quad 2.30796900-0.60403000-2.64141100$

$\begin{array}{lllll}\text { C } & 2.06966100 & -1.93901900 & -3.34009700\end{array}$

$\mathrm{H} \quad 1.82306000-2.71660500-2.60852300$

$\mathrm{H} \quad 1.23309200-1.85968400-4.04253400$

H $\quad 2.95688100 \quad-2.26294300 \quad-3.89692500$

C $\quad-0.27687200 \quad-1.62846700 \quad 4.08667300$

C $\quad-1.53785000 \quad-2.29711400 \quad 4.64614400$

C $\quad 0.75589000 \quad-1.42630200 \quad 5.19987700$

H $\quad 0.14131400-2.31315100 \quad 3.33145100$

H $\quad-2.27267500 \quad-2.46435700 \quad 3.85284800$

H $\quad-1.29975800 \quad-3.26438500 \quad 5.10321600$

H $\quad-1.99884300 \quad-1.65740600 \quad 5.40599900$

H $\quad \begin{array}{llll}1.67210600 & -0.96150500 & 4.82008700\end{array}$

$\begin{array}{llll}\mathrm{H} & 0.34674600 & -0.76298500 & 5.96890800\end{array}$

$\begin{array}{lllll}H & 1.02336300 & -2.37902200 & 5.66966000\end{array}$

$\begin{array}{llll}\text { C } & -2.13717600 & 1.78445300 & 2.24378200\end{array}$

C $\quad-1.61653500 \quad 2.62988000 \quad 3.41222900$

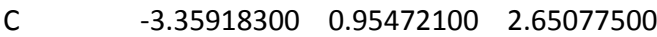

$\begin{array}{llll}\mathrm{H} & -2.47468000 & 2.48419700 & 1.46328000\end{array}$

$\begin{array}{llll}\text { H } & -0.78248100 & 3.26690200 & 3.09433100\end{array}$

$\begin{array}{llll}\mathrm{H} & -2.40741300 & 3.28145700 & 3.80053600\end{array}$

H $\quad-1.26377600 \quad 1.97944400 \quad 4.21553200$

$\begin{array}{llll}\mathrm{H} & -3.73699100 & 0.37837700 & 1.79924000\end{array}$

$\begin{array}{llll}\mathrm{H} & -3.10207800 & 0.26264000 & 3.45502700\end{array}$

H $\quad-4.16484400 \quad 1.61043500 \quad 2.99912200$

$\begin{array}{llll}\mathrm{H} & -0.36539000 & 2.48078300 & 0.56748300\end{array}$

$\begin{array}{lllll}\text { C } & 0.45861700 & 3.85827300 & -0.68700300\end{array}$

$\begin{array}{lllll}\text { C } & 0.51988400 & 4.03996400 & -2.18820600\end{array}$

H $\quad 0.97641500 \quad 3.16787200 \quad-2.66678300$

$\begin{array}{lllll}H & -0.48248400 & 4.18508200 & -2.60245300\end{array}$

$\mathrm{H} \quad 1.12202100 \quad 4.91879100 \quad-2.43743700$

$\begin{array}{lllll}0 & -0.35418100 & 2.71793700 & -0.39275200\end{array}$

H $\quad 1.78277600 \quad-1.33677100 \quad 2.60202200$

H $\quad 1.95360900 \quad-1.78616400 \quad 0.89814300$

$\begin{array}{llll}\mathrm{H} & 1.46510900 & 3.71048500 & -0.27542100\end{array}$

H $\quad 0.01889100 \quad 4.74102300 \quad-0.20610800$

H $\quad 3.18503200 \quad-0.70157200-1.97522700$

$\mathrm{H} \quad 2.58886500 \quad 0.14802500 \quad-3.39969000$

H $\quad-4.47345500 \quad-0.53288500-2.44655700$

\section{$\mathrm{TS}_{3 \mathrm{~A}-4 \mathrm{~A}}$ (EtOH)}

$H=-1034.181219 a u$

N $\quad \begin{array}{llll}-1.09619100 & 1.10414000 & 1.59497600\end{array}$

$\begin{array}{lllll}\text { C } & & -0.44107500 & 0.16332800 & 2.31603200\end{array}$

N $\quad-0.61379400 \quad-0.19792600 \quad 3.52268400$

$\begin{array}{lllll}\text { O } & 0.48764100 & -0.48627600 & 1.40904500\end{array}$

$\begin{array}{llll}\text { C } & 1.86985500 & -0.61193800 & 1.80920300\end{array}$

$\begin{array}{llll}C & 2.76481600 & 0.21770500 & 0.90915000\end{array}$

$\begin{array}{llll}\mathrm{H} & 2.54102000 & 1.28379300 & 1.01291300\end{array}$

$\begin{array}{llll}H & 3.81363300 & 0.06210900 & 1.18321600\end{array}$

$\mathrm{H} \quad 2.63952400-0.06843400-0.14098300$

U $\quad \begin{array}{llll}-0.49666700 & 0.17798400 & -0.92159100\end{array}$

$\begin{array}{lllll}0 & -2.40366700 & -0.43449100 & -1.82584700\end{array}$

$\begin{array}{lllll}0 & 1.20243000 & -0.51314400 & -2.15582700\end{array}$

C $\quad-3.54931000-0.91331500 \quad-2.45561600$

C $\quad-3.94676200 \quad-2.29552300 \quad-1.94806600$

H $\quad-4.13738600-2.26737700 \quad-0.86977400$

H $\quad-4.85365500 \quad-2.65676100 \quad-2.44695200$

H $\quad-3.14417000 \quad-3.01786100-2.13337600$

C $\quad 2.15624700 \quad-1.08274700 \quad-2.99452200$

C $\quad 1.78566000 \quad-2.50219900-3.41136400$

$\mathrm{H} \quad 1.68573500-3.14670500-2.53085700$

$\mathrm{H} \quad 0.82919300-2.50722300 \quad-3.94478700$

$\mathrm{H} \quad 2.54823500-2.93691300 \quad-4.06823400$

C $\quad-0.10934700 \quad-1.42803200 \quad 4.10227300$

C $\quad-1.30098800-2.32052500 \quad 4.46727300$

C $\quad 0.71946400 \quad-1.10795000 \quad 5.34918800$

$\mathrm{H} \quad 0.51853900 \quad-1.99873000 \quad 3.39971000$

H $\quad-1.88695300 \quad-2.57096200 \quad 3.57767800$

H $\quad-0.96241200 \quad-3.25455800 \quad 4.92946600$

H $\quad-1.95941500 \quad-1.80261300 \quad 5.17217500$

H $\quad 1.59102900-0.49036700 \quad 5.10757300$

$\begin{array}{llll}\mathrm{H} & 0.11038000 & -0.55019500 & 6.06781200\end{array}$

$\mathrm{H} \quad 1.07388300 \quad-2.02506800 \quad 5.83170600$

$\begin{array}{lllll}\text { C } & -2.33092800 & 1.69734000 & 2.13800600\end{array}$

$\begin{array}{llll}\text { C } & -2.07869700 & 2.57453100 & 3.36883400\end{array}$

$\begin{array}{lllll}\text { C } & -3.43180100 & 0.66491700 & 2.38922600\end{array}$

H $\quad-2.68458500 \quad 2.35787100 \quad 1.33254200$

$\begin{array}{llll}\mathrm{H} & -1.32242900 & 3.33761500 & 3.15446000\end{array}$

H $\quad-2.99965900 \quad 3.08704400 \quad 3.66854000$

H $\quad-1.72576900 \quad 1.96334900 \quad 4.20230400$

$\begin{array}{llll}\mathrm{H} & -3.62324000 & 0.07610600 & 1.48563400\end{array}$

$\begin{array}{llll}\mathrm{H} & -3.14905000 & -0.01168300 & 3.19868000\end{array}$

H $\quad-4.36456000 \quad 1.16661100 \quad 2.66850700$

$\begin{array}{lllll}\text { H } & -0.45307900 & 2.06109800 & 0.76777500\end{array}$

$\begin{array}{lllll}\text { C } & 0.30588800 & 3.84960000 & -0.22667600\end{array}$

$\begin{array}{llll}\text { C } & 0.75146500 & 4.24343000 & -1.62266700\end{array}$

H $\quad \begin{array}{llll}\mathrm{C} & 1.58116800 & 3.61259000 & -1.95756300\end{array}$

H $\quad-0.07213700 \quad 4.13922400 \quad-2.33670000$

H $\quad 1.08575700 \quad 5.28588700 \quad-1.64140300$

$\begin{array}{llll}\mathrm{O} & -0.11485600 & 2.49890400 & -0.20684300\end{array}$

$\begin{array}{llll}\mathrm{H} & 1.95369500 & -0.29231000 & 2.85056800\end{array}$

$\begin{array}{llll}H & 2.12911700 & -1.67461600 & 1.75346400\end{array}$

$\begin{array}{llll}\mathrm{H} & 1.13352600 & 3.98983100 & 0.48400300\end{array}$

$\begin{array}{lllll}\mathrm{H} & -0.51847500 & 4.49912100 & 0.10132900\end{array}$

H $\quad 3.14321200-1.10184600 \quad-2.49786000$

H $\quad 2.28476600-0.46581000 \quad-3.90027600$

$\mathrm{H} \quad-4.39132900-0.21825900 \quad-2.29581200$

$\mathrm{H} \quad-3.39505000 \quad-0.96407400 \quad-3.54720000$ 


\section{Int-4A (EtOH)}

$H=-1034.190808$ au

$\begin{array}{llll}\mathrm{N} & -0.66954900 & 0.96499200 & 1.87151100\end{array}$

$\begin{array}{llll}\text { C } & 0.06509800 & -0.00189600 & 2.52082800\end{array}$

N $\quad \begin{array}{llll}\text { N } & -0.18002100 & -0.46645700 & 3.67046000\end{array}$

$\begin{array}{lllll}\text { O } & 1.03878600 & -0.46407100 & 1.60989000\end{array}$

$\begin{array}{lllll}\text { C } & 2.42109600 & -0.56768300 & 2.04118800\end{array}$

$\begin{array}{lllll}\text { C } & 3.30831700 & 0.18977600 & 1.07579300\end{array}$

$\begin{array}{llll}\mathrm{H} & 3.07528200 & 1.25821600 & 1.08216700\end{array}$

$\begin{array}{llll}\mathrm{H} & 4.35640600 & 0.06286400 & 1.36606800\end{array}$

H $\quad 3.18993900 \quad-0.18855500 \quad 0.05397200$

$\begin{array}{lllll}\text { U } & 0.10367100 & 0.02801600 & -0.87760100\end{array}$

O $\quad-1.83847500-0.45637400 \quad-1.81861200$

$\begin{array}{lllll}0 & 1.81443600 & -0.77034900 & -2.03978800\end{array}$

C $\quad-3.00519600 \quad-0.72698800 \quad-2.52267800$

C $\quad-3.56125800-2.11070700-2.19867300$

H $\quad-3.78262300-2.19315000-1.12875000$

H $\quad-4.48393600 \quad-2.31203300-2.75602500$

$\mathrm{H} \quad-2.83026400 \quad-2.88565000 \quad-2.45394500$

C $\quad 2.71069200 \quad-1.28779100-2.96830100$

$\begin{array}{llll}\text { C } & 2.30807200 & -2.67856100 & -3.44962000\end{array}$

H $\quad 2.25442200 \quad-3.37501600 \quad-2.60538300$

H $\quad 1.32196900-2.64816400 \quad-3.92549200$

H $\quad 3.02809300 \quad-3.07490300-4.17556100$

$\begin{array}{lllll}\text { C } & 0.38449500 & -1.70273100 & 4.18695800\end{array}$

$\begin{array}{lllll}\text { C } & -0.75957700 & -2.69551500 & 4.41335600\end{array}$

C $\quad 1.12265500 \quad-1.41540500 \quad 5.49560800$

H $\quad \begin{array}{llll}\text { H } & 1.08783200 & -2.16568500 & 3.47878600\end{array}$

H $\quad-1.28103100 \quad-2.91349800 \quad 3.47647500$

H $\quad-0.37688700 \quad-3.63771700 \quad 4.81973300$

$\begin{array}{llll}\mathrm{H} & -1.48699700 & -2.28278400 & 5.11959800\end{array}$

H $\quad \begin{array}{llll}H & 1.95942500 & -0.72632700 & 5.34283200\end{array}$

$\begin{array}{llll}H & 0.44090600 & -0.95550100 & 6.21785500\end{array}$

H $\quad \begin{array}{llll}H & 1.51653900 & -2.34004900 & 5.92957900\end{array}$

$\begin{array}{llll}\text { C } & -1.91434800 & 1.53511200 & 2.41839400\end{array}$

$\begin{array}{llll}\text { C } & -1.68264600 & 2.34223900 & 3.69748900\end{array}$

$\begin{array}{lllll}C & -2.99665600 & 0.47120600 & 2.58289700\end{array}$

$\begin{array}{llll}\mathrm{H} & -2.24100800 & 2.22497700 & 1.63083400\end{array}$

$\begin{array}{llll}\mathrm{H} & -0.93276700 & 3.12396800 & 3.53718800\end{array}$

$\begin{array}{llll}H & -2.61322400 & 2.82845600 & 4.00818600\end{array}$

$\begin{array}{llll}\mathrm{H} & -1.34125200 & 1.68919800 & 4.50365400\end{array}$

H $\quad-3.14878400-0.07458500 \quad 1.64644000$

$\begin{array}{llll}\mathrm{H} & -2.73140500 & -0.23802900 & 3.36959500\end{array}$

$\begin{array}{llll}\mathrm{H} & -3.94289300 & 0.94925800 & 2.85433200\end{array}$

H $\quad-0.12513400 \quad 1.64304400 \quad 1.31521600$

C $\quad 0.65993700 \quad 3.56069200 \quad-0.81327800$

$\begin{array}{lllll}\text { C } & 1.02033200 & 3.72085300 & -2.28642000\end{array}$

$\mathrm{H} \quad 1.91045000 \quad 3.13041000 \quad-2.53032100$

$\begin{array}{llll}\mathrm{H} & 0.19666900 & 3.37472400 & -2.92152300\end{array}$

H $\quad 1.22475900 \quad 4.76821800 \quad-2.53782800$

$\begin{array}{lllll}0 & 0.39608200 & 2.23520600 & -0.47141700\end{array}$

H $\quad 2.49030700 \quad-0.16860900 \quad 3.05683700$

H $\quad 2.68026600 \quad-1.63083600 \quad 2.06756400$

H $\quad 1.48958400 \quad 3.95749700 \quad-0.20101700$

H $\quad-0.21409800 \quad 4.19885900 \quad-0.59134900$

H $\quad 3.72532800 \quad-1.34220000 \quad-2.53341000$

H $\quad 2.79345700 \quad-0.61851900-3.84243200$

H $\quad-3.77893000 \quad 0.02846700 \quad-2.29705600$

H $\quad-2.82657500 \quad-0.66340100 \quad-3.61067500$

pre-Cat $_{\mathrm{B}}$

$H=-942.447023 a u$ $\begin{array}{lll}0.16052800 & 0.67549600 & 0.34366600\end{array}$ $\begin{array}{lll}0.37553500 & 3.84719500 & 1.97043300\end{array}$

$\begin{array}{lll}2.41099700 & 1.75914400 & 2.89974500\end{array}$

$\begin{array}{lll}-3.12858900 & 0.55719300 & 1.13334600\end{array}$

$\begin{array}{lll}-2.74051500 & 1.02279500 & -1.88776400\end{array}$

$\begin{array}{lll}1.66001800 & -1.60332900 & -1.07563100\end{array}$

$\begin{array}{llll}2.66822000 & 1.12343400 & -2.38748000\end{array}$

$\begin{array}{lll}1.04781800 & 2.21189300 & 1.86636100\end{array}$

$\begin{array}{lll}-2.07786600 & 0.84835400 & -0.25487800\end{array}$

$\begin{array}{llll}1.62463200 & 0.17723800 & -1.32816000\end{array}$

$\begin{array}{llll}2.37470300 & 0.72891700 & -4.22093800\end{array}$

$\begin{array}{llll}1.33349000 & 0.90125100 & -4.50929900\end{array}$

$\begin{array}{llll}3.00763700 & 1.36345200 & -4.85187100\end{array}$

$\begin{array}{lll}2.61826000 & -0.31347100 & -4.45014900\end{array}$

$\begin{array}{llll}4.51507600 & 0.85325600 & -2.03531900\end{array}$

$\begin{array}{lll}4.84529500 & -0.15009500 & -2.31891300\end{array}$

$\begin{array}{llll}5.11130800 & 1.57183000 & -2.60940100\end{array}$

$\begin{array}{llll}4.74814000 & 0.99596300 & -0.97553200\end{array}$

$\begin{array}{llll}2.31145000 & 2.96835300 & -2.10706700\end{array}$

$\begin{array}{llll}1.28920300 & 3.23483400 & -2.39518300\end{array}$

$\begin{array}{llll}2.46389600 & 3.24761800 & -1.05851800\end{array}$

$\begin{array}{llll}2.98937400 & 3.58180100 & -2.71096000\end{array}$

$\begin{array}{lll}-1.44083300 & 1.87299300 & -2.97464700\end{array}$

$\begin{array}{llll}-0.50714800 & 1.30087500 & -3.00272600\end{array}$

$\begin{array}{llll}-1.80392400 & 1.95728100 & -4.00492900\end{array}$

$\begin{array}{lll}-1.22031900 & 2.88475200 & -2.61792600\end{array}$

$\begin{array}{llll}-4.31651500 & 2.08113700 & -1.93880100\end{array}$

$\begin{array}{llll}-4.62524300 & 2.22487200 & -2.98064700\end{array}$

$\begin{array}{llll}-5.15210500 & 1.60632800 & -1.41449400\end{array}$

$\begin{array}{llll}-4.15484000 & 3.06949400 & -1.49762700\end{array}$

$\begin{array}{llll}-3.15123200 & -0.65637200 & -2.67160000\end{array}$

$-3.93142800-1.18354800-2.11427100$

$\begin{array}{lll}-3.50668700 & -0.52218400 & -3.69967000\end{array}$

$\begin{array}{llll}-2.27076100 & -1.30575300 & -2.70505200\end{array}$

$\begin{array}{lll}-1.99828100 & 0.11378200 & 2.61740000\end{array}$

$\begin{array}{lll}-1.45193300 & -0.82795000 & 2.47447100\end{array}$

$\begin{array}{llll}-1.29176100 & 0.90171900 & 2.91428500\end{array}$

$\begin{array}{lll}-2.64517300 & -0.03801900 & 3.48920500\end{array}$

$\begin{array}{lll}-4.30924400 & -0.90638400 & 0.89521300\end{array}$

$\begin{array}{lll}-4.85818100 & -1.10488200 & 1.82265500\end{array}$

$\begin{array}{lll}-5.04806500 & -0.70518500 & 0.11298700\end{array}$

$\begin{array}{lll}-3.76482600 & -1.81516000 & 0.62097700\end{array}$

$\begin{array}{lll}-4.14709500 & 2.06725000 & 1.66019000\end{array}$

$\begin{array}{lll}-4.68285600 & 1.85468400 & 2.59246300\end{array}$

$\begin{array}{lll}-3.51128600 & 2.94149800 & 1.82971100\end{array}$

$\begin{array}{lll}-4.88959500 & 2.33691100 & 0.90411500\end{array}$

$\begin{array}{lll}-0.64415500 & 4.19731000 & 0.40145800\end{array}$

$\begin{array}{lll}-1.12040700 & 5.17966100 & 0.50123600\end{array}$

$\begin{array}{llll}-0.00672900 & 4.23948100 & -0.48866000\end{array}$

$\begin{array}{llll}-1.44584900 & 3.47155600 & 0.22192900\end{array}$

$\begin{array}{lll}-0.77970100 & 4.06988700 & 3.46152900\end{array}$

$\begin{array}{llll}-0.24572400 & 3.95046400 & 4.40860100\end{array}$

$\begin{array}{llll}-1.22145800 & 5.07297400 & 3.45177700\end{array}$

$\begin{array}{lll}-1.60043100 & 3.34584900 & 3.44886100\end{array}$

$\begin{array}{lll}1.70073800 & 5.20410200 & 2.06154800\end{array}$

$\begin{array}{llll}2.39075600 & 5.14740600 & 1.21410800\end{array}$

$\begin{array}{lll}1.21750600 & 6.18757700 & 2.03464100\end{array}$

$\begin{array}{llll}2.28914500 & 5.15411900 & 2.98292500\end{array}$

$\begin{array}{llll}2.59956400 & -0.13362800 & 2.88800400\end{array}$

$\begin{array}{lll}1.71744200 & -0.65273700 & 3.27739500\end{array}$

$\begin{array}{llll}2.82901600 & -0.54064300 & 1.89725100\end{array}$

$\begin{array}{llll}3.44337600 & -0.39951200 & 3.53536400\end{array}$

$\begin{array}{llll}2.17306200 & 2.26417400 & 4.71532200\end{array}$ 
$\begin{array}{lll}2.15590000 & 3.35036000 & 4.84982500\end{array}$ $\begin{array}{lll}1.24254600 & 1.85707000 & 5.12340600\end{array}$ $\begin{array}{lll}3.00232200 & 1.87402400 & 5.31669800\end{array}$ $\begin{array}{lll}4.06289800 & 2.47150100 & 2.29596300\end{array}$ $\begin{array}{lll}4.88711100 & 2.09408200 & 2.91212900\end{array}$ $\begin{array}{lll}4.25916100 & 2.17926300 & 1.25936900\end{array}$ $\begin{array}{llll}4.08249700 & 3.56357500 & 2.34512200\end{array}$ $0.96470200-2.54542300-2.56677800$ $-0.05051700-2.21498200-2.80578800$ $\begin{array}{llll}1.58190800 & -2.40080700 & -3.45945700\end{array}$ $\begin{array}{lll}0.92859000 & -3.61910400 & -2.35134900\end{array}$ $3.40045100-2.26974000-0.71801300$ $\begin{array}{llll}4.03165900 & -2.24245600 & -1.61163400\end{array}$ $\begin{array}{lll}3.90843200 & -1.70628600 & 0.06993500\end{array}$ $\begin{array}{lll}3.32565600 & -3.31382800 & -0.39425400\end{array}$ $\begin{array}{llll}0.54224200 & -1.73086700 & 0.46001100\end{array}$ $\begin{array}{lll}1.06312100 & -2.20343200 & 1.29802600\end{array}$ $\begin{array}{lll}-0.37646100 & -2.29579900 & 0.25493600\end{array}$

\section{dimer-B (EtOH)}

$H=-1310.038493 a u$

$\begin{array}{llll}\text { O } & -0.30199500 & 1.28592000 & 2.47675100\end{array}$

$\begin{array}{lllll}0 & -1.92646600 & 2.64764800 & 0.94395200\end{array}$

$\begin{array}{lllll}\text { U } & -1.24789400 & 3.39207900 & 3.11575900\end{array}$

$\begin{array}{llll}\text { C } & -2.76399500 & 3.45182300 & 0.11152000\end{array}$

$\begin{array}{llll}\text { C } & -4.18105600 & 2.90842000 & 0.04495200\end{array}$

H $\quad-4.19796800 \quad 1.89935500 \quad-0.38070000$

$\begin{array}{llll}\mathrm{H} & -4.62328700 & 2.87033800 & 1.04492100\end{array}$

H $\quad-4.80869100 \quad 3.54897200 \quad-0.58449900$

$\begin{array}{lllll}\text { C } & 0.53173800 & 0.47478700 & 3.30537500\end{array}$

$\begin{array}{llll}\text { C } & 1.94263200 & 1.02923300 & 3.40468700\end{array}$

$\begin{array}{llll}\mathrm{H} & 2.40001400 & 1.09217200 & 2.41289400\end{array}$

$\begin{array}{llll}\mathrm{H} & 1.94392100 & 2.02876000 & 3.85258900\end{array}$

H $\quad 2.56648800 \quad 0.38076600 \quad 4.02978100$

$\begin{array}{lllll}\text { U } & -0.96830200 & 0.55181100 & 0.29704100\end{array}$

$\begin{array}{lllll}0 & -1.18501500 & 1.18219200 & -1.74698800\end{array}$

$\begin{array}{lllll}0 & -2.45941500 & -0.92961700 & 0.67552500\end{array}$

$\begin{array}{lllll}0 & 0.91568500 & -0.46739800 & 0.11215000\end{array}$

$\begin{array}{lllll}0 & -3.12151200 & 4.42848600 & 3.31667300\end{array}$

$\begin{array}{lllll}0 & 0.24939900 & 4.86006300 & 2.71444700\end{array}$

$\begin{array}{lllll}0 & -1.00325900 & 2.78200600 & 5.16246600\end{array}$

C $\quad-3.32218800-2.02985900 \quad 0.66889600$

$\begin{array}{llll}\text { C } & -4.18048700 & -2.07007000 & 1.92541600\end{array}$

H $\quad-4.78618100 \quad-1.16187000 \quad 2.00599200$

H $\quad-4.85484500 \quad-2.93365800 \quad 1.90941900$

$\begin{array}{llll}H & -3.55131300 & -2.14126800 & 2.81841700\end{array}$

C $\quad 2.19197500 \quad-0.98719700 \quad-0.10884800$

C $\quad 2.21434700-1.94768700 \quad-1.29034000$

H $\quad 3.22126200-2.35028000-1.44908800$

H $\quad \begin{array}{llll}\text { H } & 1.53204800 & -2.78579600 & -1.11563700\end{array}$

H $\quad \begin{array}{llll}1.89892000 & -1.43679800 & -2.20555600\end{array}$

C $\quad-1.09437800 \quad 1.61494500 \quad-3.07108300$

$\begin{array}{lllll}\text { C } & -4.36540700 & 4.98785800 & 3.61191400\end{array}$

$\begin{array}{llll}\text { C } & 1.13822300 & 5.93943900 & 2.70332800\end{array}$

C $\quad \begin{array}{llll}\text { C } & 2.18903200 & 5.78289700 & 1.61312800\end{array}$

$\begin{array}{llll}\mathrm{H} & 1.71482600 & 5.72339300 & 0.62825700\end{array}$

$\begin{array}{llll}\mathrm{H} & 2.87935800 & 6.63391400 & 1.61226500\end{array}$

$\begin{array}{llll}\mathrm{H} & 2.77027600 & 4.86841500 & 1.76816300\end{array}$

$\begin{array}{llll}\text { C } & -1.04348500 & 2.33209000 & 6.48309500\end{array}$

$\begin{array}{llll}C & -2.31495500 & 1.54860900 & 6.78380000\end{array}$

H $\quad-3.19872100 \quad 2.17227100 \quad 6.61435100$

$\begin{array}{lllll}H & -2.38890200 & 0.66835600 & 6.13605700\end{array}$

$\begin{array}{llll}H & -2.32982200 & 1.20782700 & 7.82523300\end{array}$ $\begin{array}{lll}-2.32551600 & 3.49452200 & -0.89332200\end{array}$ $\begin{array}{lll}-2.78609000 & 4.47810000 & 0.50490400\end{array}$ $\begin{array}{lll}0.07956100 & 0.40791100 & 4.30283800\end{array}$ $\begin{array}{lll}0.56997000 & -0.54364000 & 2.89288000\end{array}$ $\begin{array}{lll}-0.16555500 & 1.69810200 & 6.68779200\end{array}$ $\begin{array}{lll}-0.97296700 & 3.19207100 & 7.16724000\end{array}$ $\begin{array}{llll}-4.85314100 & 4.41495200 & 4.41712400\end{array}$ $\begin{array}{lll}1.63339200 & 6.02452800 & 3.68326500\end{array}$ $\begin{array}{lll}0.58188100 & 6.87676300 & 2.54721500\end{array}$ $\begin{array}{lll}2.54058000 & -1.51172900 & 0.79541800\end{array}$ $\begin{array}{lll}2.90708900 & -0.16826500 & -0.28995300\end{array}$ $\begin{array}{llll}-0.04779100 & 1.56055200 & -3.41133900\end{array}$ $\begin{array}{llll}-1.39089100 & 2.67446900 & -3.14136700\end{array}$ $\begin{array}{lll}-2.73882600 & -2.96068700 & 0.59106600\end{array}$ $\begin{array}{lll}-3.97135900 & -1.98824000 & -0.21970200\end{array}$ $\begin{array}{lll}-1.97161300 & 0.78342000 & -3.99721100\end{array}$ $\begin{array}{llll}-1.89084400 & 1.13427300 & -5.03235000\end{array}$ $\begin{array}{lll}-1.67199400 & -0.26900400 & -3.96561500\end{array}$ $\begin{array}{llll}-3.02106300 & 0.84759500 & -3.69252800\end{array}$ $\begin{array}{lll}-4.24153300 & 6.44626400 & 4.03235000\end{array}$ $\begin{array}{lll}-5.22476000 & 6.87407800 & 4.25900300\end{array}$ $\begin{array}{lll}-3.61295400 & 6.53550900 & 4.92423900\end{array}$ $\begin{array}{lll}-3.78398800 & 7.03815300 & 3.23307700\end{array}$ $\begin{array}{lll}-5.02574000 & 4.91628500 & 2.73245500\end{array}$

\section{$\mathrm{Cat}_{\mathrm{B}}$ (EtOH)}

$H=-654.990954 a u$

C $\quad 3.73213000 \quad 0.75789500 \quad-3.54137800$

H $\quad 3.99203100 \quad 1.82058300 \quad-3.49808800$

$\begin{array}{lllll}\mathrm{H} & 4.51341900 & 0.23544100 & -4.10475800\end{array}$

$\begin{array}{llll}\mathrm{H} & 2.78968500 & 0.65863900 & -4.08988900\end{array}$

$\begin{array}{lllll}\text { C } & -2.31383500 & 0.77193000 & -2.71877200\end{array}$

H $\quad \begin{array}{lllll}\mathrm{H} & -1.54691900 & 0.23107500 & -3.28287200\end{array}$

$\begin{array}{llll}\mathrm{H} & -3.29384100 & 0.36998800 & -2.99940300\end{array}$

H $\quad-2.27736200 \quad 1.82543900 \quad-3.01488500$

$\begin{array}{llll}\text { C } & 1.48107300 & 0.04742200 & 3.50373100\end{array}$

H $\quad 0.48687100 \quad-0.32961600 \quad 3.24229800$

$\begin{array}{llll}H & 2.22585300 & -0.55089200 & 2.96871600\end{array}$

H $\quad \begin{array}{llll}\text { H } & 1.63175500 & -0.09875800 & 4.57922200\end{array}$

$\begin{array}{lllll}\text { O } & 2.60746300 & 0.83984700 & -1.40153700\end{array}$

$\begin{array}{lllll}0 & 1.41845400 & 1.72552400 & 1.76287100\end{array}$

$\begin{array}{lllll}0 & -0.82966400 & 1.13687000 & -0.84468100\end{array}$

$\begin{array}{llll}\text { C } & -2.07505900 & 0.62959000 & -1.22172400\end{array}$

C $\quad 3.59653700 \quad 0.18359200 \quad-2.13766700$

$\begin{array}{llll}\text { C } & 1.60936400 & 1.51812500 & 3.13084800\end{array}$

$\begin{array}{lllll}\text { O } & 1.18806900 & 3.99381000 & -0.88006900\end{array}$

C $\quad 1.21392200 \quad 5.36161300 \quad-1.16173600$

$\begin{array}{lllll}\text { C } & 0.26031400 & 6.14163000 & -0.26689700\end{array}$

$\begin{array}{llll}\mathrm{H} & -0.76828600 & 5.79254700 & -0.40493800\end{array}$

$\begin{array}{llll}\mathrm{H} & 0.52795000 & 6.00965400 & 0.78658500\end{array}$

H $\quad 0.29305000 \quad 7.21223500 \quad-0.49817000$

H $\quad 4.56161700 \quad 0.26218100 \quad-1.61287500$

H $\quad 3.35938900 \quad-0.89005500 \quad-2.20024600$

H $\quad 2.23769300 \quad 5.74659800 \quad-1.03278400$

H $\quad 0.94682600 \quad 5.52672400 \quad-2.21733200$

$\begin{array}{llll}\mathrm{H} & 2.60424100 & 1.88737700 & 3.42537200\end{array}$

$\begin{array}{llll}\mathrm{H} & 0.87400800 & 2.10791500 & 3.70034100\end{array}$

H $\quad-2.14375900-0.43218000 \quad-0.93724000$

H $\quad-2.87088800 \quad 1.15456800 \quad-0.67031000$

U $\quad 1.09602000 \quad 1.92450000 \quad-0.34396600$

\section{Int-1B (EtOH)}




\begin{tabular}{|c|c|c|c|}
\hline \multicolumn{4}{|c|}{$H=-1039.321514 a u$} \\
\hline N & -0.29174200 & 2.16353900 & 0.46726100 \\
\hline C & -0.71202400 & 2.29997600 & 1.62912300 \\
\hline $\mathrm{N}$ & -0.99483000 & 2.41304700 & 2.80120700 \\
\hline 0 & 1.20550600 & -0.20393100 & 1.95536600 \\
\hline C & 1.83812200 & -0.03529800 & 3.18475800 \\
\hline C & 3.35271100 & 0.05927200 & 3.04482200 \\
\hline $\mathrm{H}$ & 3.62922900 & 0.91537400 & 2.42002300 \\
\hline $\mathrm{H}$ & 3.83266100 & 0.18065100 & 4.02289700 \\
\hline $\mathrm{H}$ & 3.75084100 & -0.84727300 & 2.57713900 \\
\hline$U$ & 0.45028800 & -0.47242000 & -0.04688400 \\
\hline 0 & -1.69681900 & -0.78401500 & -0.07016400 \\
\hline 0 & 1.35598800 & 0.41491200 & -1.82071600 \\
\hline C & -2.22064100 & 2.06013000 & 3.52112700 \\
\hline C & -3.32413200 & 1.55507700 & 2.59680100 \\
\hline C & -2.65330300 & 3.25689100 & 4.36416300 \\
\hline $\mathrm{H}$ & -1.92438500 & 1.24990800 & 4.19971200 \\
\hline $\mathrm{H}$ & -2.97243000 & 0.72034300 & 1.98269900 \\
\hline $\mathrm{H}$ & -4.18336400 & 1.21600100 & 3.18366200 \\
\hline $\mathrm{H}$ & -3.66532500 & 2.35572000 & 1.93071800 \\
\hline $\mathrm{H}$ & -1.83733300 & 3.59513600 & 5.00807600 \\
\hline $\mathrm{H}$ & -2.95488300 & 4.09202300 & 3.72352600 \\
\hline $\mathrm{H}$ & -3.50475000 & 2.98396300 & 4.99467100 \\
\hline C & -0.41109100 & 3.28179300 & -0.51005000 \\
\hline C & -1.17176800 & 2.79848000 & -1.73914800 \\
\hline C & 0.99054100 & 3.77468000 & -0.85332400 \\
\hline $\mathrm{H}$ & -0.97475700 & 4.09673800 & -0.03593600 \\
\hline $\mathrm{H}$ & -2.16377600 & 2.42585900 & -1.46898900 \\
\hline $\mathrm{H}$ & -1.29293900 & 3.62144300 & -2.45090000 \\
\hline $\mathrm{H}$ & -0.61402500 & 1.99498500 & -2.23021100 \\
\hline $\mathrm{H}$ & 1.50586900 & 4.14777100 & 0.03657000 \\
\hline $\mathrm{H}$ & 1.57324500 & 2.95283200 & -1.27869500 \\
\hline $\mathrm{H}$ & 0.93598500 & 4.58637700 & -1.58612700 \\
\hline 0 & 1.11390600 & -2.47976800 & -0.37048200 \\
\hline C & 1.61618100 & -3.77659500 & -0.45299800 \\
\hline C & 0.50853400 & -4.80342900 & -0.65085300 \\
\hline $\mathrm{H}$ & 0.92065100 & -5.81707700 & -0.71497200 \\
\hline $\mathrm{H}$ & -0.19851100 & -4.77066700 & 0.18427800 \\
\hline $\mathrm{H}$ & -0.04452000 & -4.59776900 & -1.57301100 \\
\hline C & 2.10557600 & 0.54666500 & -2.98967800 \\
\hline C & 3.59618800 & 0.34298800 & -2.74627600 \\
\hline $\mathrm{H}$ & 4.16707000 & 0.46272700 & -3.67436400 \\
\hline $\mathrm{H}$ & 3.97072700 & 1.07012700 & -2.01782900 \\
\hline $\mathrm{H}$ & 3.78720300 & -0.66129600 & -2.35394100 \\
\hline C & -2.92696700 & -1.33373200 & -0.42825000 \\
\hline C & -3.00007500 & -1.68036700 & -1.91073000 \\
\hline $\mathrm{H}$ & -2.84300500 & -0.78585700 & -2.52309700 \\
\hline $\mathrm{H}$ & -2.22865700 & -2.41274900 & -2.17148200 \\
\hline $\mathrm{H}$ & -3.97643100 & -2.10574500 & -2.17033800 \\
\hline $\mathrm{H}$ & 1.58833000 & -0.87806800 & 3.85028800 \\
\hline $\mathrm{H}$ & 1.45853700 & 0.87544400 & 3.67550700 \\
\hline $\mathrm{H}$ & 2.17978100 & -4.01866100 & 0.46262300 \\
\hline $\mathrm{H}$ & 1.94430600 & 1.54835000 & -3.42379900 \\
\hline $\mathrm{H}$ & 1.75972600 & -0.17666500 & -3.74630000 \\
\hline $\mathrm{H}$ & 2.33093300 & -3.84562100 & -1.28908000 \\
\hline $\mathrm{H}$ & -3.12192600 & -2.24132500 & 0.16660600 \\
\hline $\mathrm{H}$ & -3.73563100 & -0.62431800 & -0.18348300 \\
\hline
\end{tabular}

\section{$\mathrm{TS}_{1 \mathrm{~B}-2 \mathrm{~B}}$ (EtOH)}

$H=-1039.308508 \mathrm{au}$

$\begin{array}{llll}\mathrm{N} & -1.01005700 & 2.40486600 & 0.75769500\end{array}$

$\begin{array}{llll}\text { C } & -1.21190200 & 2.43046100 & 2.02708200\end{array}$ $\begin{array}{lll}-1.48777900 & 3.05680700 & 3.04524400\end{array}$ $\begin{array}{lll}-0.70283700 & 0.58302700 & 2.35740800\end{array}$ $\begin{array}{lll}0.08951800 & 0.38901500 & 3.50741900\end{array}$ $\begin{array}{lll}1.57959000 & 0.54868300 & 3.23051800\end{array}$ $\begin{array}{lll}1.79815600 & 1.55877000 & 2.86633700\end{array}$ $\begin{array}{lll}2.16955200 & 0.38568000 & 4.13928800\end{array}$ $\begin{array}{lll}1.91431000 & -0.17845400 & 2.48138700\end{array}$ $\begin{array}{lll}-0.36154100 & 0.03138600 & 0.10986700\end{array}$ $\begin{array}{lll}-2.19845000 & -0.60644800 & -0.78034800\end{array}$ $\begin{array}{llll}0.97762200 & 0.74851000 & -1.41135300\end{array}$ $\begin{array}{lll}-2.39183600 & 2.75168000 & 4.14354300\end{array}$ $\begin{array}{lll}-3.80747900 & 3.21477200 & 3.79003500\end{array}$ $\begin{array}{lll}-1.88591200 & 3.42045000 & 5.41886900\end{array}$ $\begin{array}{lll}-2.41264500 & 1.66307000 & 4.28977100\end{array}$ $\begin{array}{lll}-4.17065300 & 2.70977000 & 2.89000900\end{array}$ $\begin{array}{lll}-4.49885800 & 2.99209200 & 4.60967500\end{array}$ $\begin{array}{lll}-3.82278600 & 4.29447800 & 3.60958000\end{array}$ $\begin{array}{lll}-0.87746900 & 3.07870600 & 5.66943300\end{array}$ $\begin{array}{lll}-1.85147700 & 4.50733000 & 5.29180200\end{array}$ $\begin{array}{lll}-2.54705700 & 3.19152000 & 6.26052000\end{array}$ $\begin{array}{llll}-1.45441300 & 3.59243500 & -0.00648000\end{array}$ $\begin{array}{llll}-1.81317000 & 3.16085900 & -1.42417800\end{array}$ $\begin{array}{llll}-0.35780000 & 4.65614800 & 0.01104400\end{array}$ $\begin{array}{llll}-2.34919700 & 4.00607300 & 0.47938200\end{array}$ $\begin{array}{llll}-2.62304200 & 2.42459200 & -1.41915100\end{array}$ $\begin{array}{llll}-2.13721100 & 4.02132900 & -2.01798600\end{array}$ $\begin{array}{llll}-0.94382000 & 2.71369000 & -1.91978100\end{array}$ $\begin{array}{lll}-0.12573600 & 4.95458900 & 1.03749900\end{array}$ $\begin{array}{llll}0.55551600 & 4.26905400 & -0.45263300\end{array}$ $\begin{array}{llll}-0.67474200 & 5.54718500 & -0.54176800\end{array}$ $\begin{array}{llll}0.67697200 & -1.76390300 & 0.67025600\end{array}$ $\begin{array}{lll}1.38977200 & -2.89367400 & 1.07126700\end{array}$ $\begin{array}{llll}0.50725600 & -4.13394000 & 1.11850900\end{array}$ $\begin{array}{llll}1.08188800 & -5.01189700 & 1.43500800\end{array}$ $\begin{array}{lll}-0.31883100 & -3.99069100 & 1.82253000\end{array}$ $\begin{array}{lll}0.08118600 & -4.33915400 & 0.13128500\end{array}$ $\begin{array}{llll}2.00154600 & 1.13828400 & -2.27540200\end{array}$ $\begin{array}{llll}1.99503900 & 0.32311000 & -3.56181400\end{array}$ $\begin{array}{llll}2.80083700 & 0.64232800 & -4.23265100\end{array}$ $\begin{array}{llll}2.13085900 & -0.74056900 & -3.34153600\end{array}$ $\begin{array}{llll}1.04171900 & 0.44314300 & -4.08643100\end{array}$ $\begin{array}{llll}-3.26248100 & -1.25220500 & -1.41406000\end{array}$ $-2.80691300-2.06676400-2.61720000$ $\begin{array}{llll}-2.32182200 & -1.42249400 & -3.35764200\end{array}$ $\begin{array}{lll}-2.09001600 & -2.83587200 & -2.31164300\end{array}$ $\begin{array}{llll}-3.65752500 & -2.56299100 & -3.09815400\end{array}$ $\begin{array}{lll}-0.11615800 & -0.61142800 & 3.91464100\end{array}$ $\begin{array}{lll}-0.21141200 & 1.11680500 & 4.27375100\end{array}$ $\begin{array}{lll}1.83374100 & -2.72402900 & 2.06613000\end{array}$ $\begin{array}{llll}2.97861200 & 1.02864500 & -1.77765500\end{array}$ $\begin{array}{lll}1.89440000 & 2.20771200 & -2.51986700\end{array}$ $\begin{array}{llll}2.22978600 & -3.06664000 & 0.37961300\end{array}$ $\begin{array}{llll}-3.77557700 & -1.91167300 & -0.69640500\end{array}$ $-4.00278300-0.50342800-1.73695900$

\section{Int-2B (EtOH)}

$H=-1039.322152 a u$

$\begin{array}{llrl}\mathrm{N} & -0.34418900 & 1.72889500 & 0.23907400 \\ \mathrm{C} & -0.65583200 & 1.53561000 & 1.54029300 \\ \mathrm{~N} & -1.09168400 & 2.38022000 & 2.38357700 \\ \mathrm{O} & -0.46169700 & 0.12298500 & 1.78543900 \\ \mathrm{C} & 0.44637900 & -0.24132600 & 2.85034900 \\ \mathrm{C} & 1.84491500 & 0.31033500 & 2.65234800\end{array}$




\begin{abstract}
$\begin{array}{lll}1.83789400 & 1.40293400 & 2.60580300\end{array}$
$\begin{array}{lll}2.47698100 & 0.01172500 & 3.49508300\end{array}$

$\begin{array}{lll}2.29695400 & -0.08712200 & 1.73836800\end{array}$

$\begin{array}{llll}0.02983500 & -0.44042500 & -0.71762300\end{array}$

$\begin{array}{lll}-1.84196400 & -1.21857000 & -1.37379700\end{array}$

$\begin{array}{llll}1.15210100 & 0.03486800 & -2.46945300\end{array}$

$\begin{array}{lll}-1.69253200 & 2.04589400 & 3.66009400\end{array}$

$\begin{array}{lll}-3.11528300 & 2.61515700 & 3.67905600\end{array}$

$\begin{array}{lll}-0.86156000 & 2.63962000 & 4.80112300\end{array}$

$\begin{array}{lll}-1.77865300 & 0.95730300 & 3.80883300\end{array}$

$\begin{array}{lll}-3.71530300 & 2.17976100 & 2.87445500\end{array}$

$\begin{array}{lll}-3.61100900 & 2.40394300 & 4.63333800\end{array}$

$\begin{array}{lll}-3.08817000 & 3.69954100 & 3.53086800\end{array}$

$\begin{array}{llll}0.15189300 & 2.22504400 & 4.81864200\end{array}$

$\begin{array}{lll}-0.77273200 & 3.72285800 & 4.66947100\end{array}$

$\begin{array}{lll}-1.32822900 & 2.44501900 & 5.77289200\end{array}$

$\begin{array}{lll}-0.60290100 & 3.05983200 & -0.31050300\end{array}$

$\begin{array}{llll}-0.88105200 & 2.95204700 & -1.80838100\end{array}$

$\begin{array}{llll}0.56309200 & 4.00683200 & -0.01824900\end{array}$

$\begin{array}{llll}-1.49387100 & 3.46223800 & 0.19162800\end{array}$

$\begin{array}{llll}-1.76081200 & 2.32666100 & -2.00227100\end{array}$

$\begin{array}{llll}-1.07524900 & 3.93899500 & -2.24046100\end{array}$

$\begin{array}{lll}-0.02474000 & 2.51980800 & -2.34040100\end{array}$

$\begin{array}{lll}0.72491600 & 4.07771700 & 1.06048400\end{array}$

$\begin{array}{llll}1.48330300 & 3.64383100 & -0.49062700\end{array}$

$0.35679800 \quad 5.01336000-0.39961600$

$\begin{array}{lll}1.21238000 & -1.99398000 & 0.18516700\end{array}$

$\begin{array}{llll}1.93711200 & -3.06262200 & 0.71394200\end{array}$

$\begin{array}{lll}1.10779100 & -4.33822400 & 0.78291300\end{array}$

$\begin{array}{lll}1.69307800 & -5.16371600 & 1.20342800\end{array}$

$\begin{array}{llll}0.22264000 & -4.19030200 & 1.41035800\end{array}$

$0.76993800-4.63115900-0.21631300$

$\begin{array}{llll}2.00328200 & 0.25733700 & -3.55582100\end{array}$

$\begin{array}{llll}3.46992600 & 0.19466600 & -3.15231200\end{array}$

$\begin{array}{llll}4.12055700 & 0.37257200 & -4.01582800\end{array}$

$\begin{array}{lll}3.69299600 & 0.95254800 & -2.39408900\end{array}$

$\begin{array}{llll}3.71294600 & -0.78821600 & -2.73587800\end{array}$

$-2.98425900-1.79272000-1.94062300$

$\begin{array}{lll}-2.66235500 & -2.56792700 & -3.21047300\end{array}$

$\begin{array}{llll}-2.21267800 & -1.90916000 & -3.96049400\end{array}$

$\begin{array}{llll}-1.95649900 & -3.37797400 & -2.99914900\end{array}$

$\begin{array}{lll}-3.56967300 & -3.00763600 & -3.63954100\end{array}$

$\begin{array}{lll}0.44813700 & -1.33334700 & 2.83823000\end{array}$

$\begin{array}{lll}0.02612100 & 0.09207000 & 3.80152100\end{array}$

$\begin{array}{lll}2.29849800 & -2.80686500 & 1.72433300\end{array}$

$\begin{array}{llll}1.78326700 & 1.24199400 & -3.99673900\end{array}$

$\begin{array}{llll}1.80229800 & -0.49015700 & -4.33834800\end{array}$

$\begin{array}{lll}2.83414000 & -3.24091900 & 0.10019600\end{array}$

$\begin{array}{llll}-3.45753000 & -2.46318700 & -1.20709200\end{array}$

$-3.71670900-1.00249700-2.16541100$
\end{abstract}

\section{Int-3B (EtOH)}

$H=-1194.240951 \mathrm{au}$

$\begin{array}{lrrr}\mathrm{N} & -0.56022500 & 0.46658200 & 1.76047900 \\ \mathrm{C} & 0.38505100 & -0.24601400 & 2.42308800 \\ \mathrm{~N} & 0.41213400 & -0.62979600 & 3.63385700 \\ \mathrm{O} & 1.39007200 & -0.55713000 & 1.42867700 \\ \mathrm{C} & 2.78116900 & -0.36258200 & 1.76311700 \\ \mathrm{C} & 3.45654300 & 0.54462700 & 0.75505100 \\ \mathrm{H} & 2.98591600 & 1.53126900 & 0.73840200 \\ \mathrm{H} & 4.51182800 & 0.66384300 & 1.02318000 \\ \mathrm{H} & 3.40447400 & 0.12250400 & -0.25189200 \\ \mathrm{U} & -0.14167300 & -0.06516900 & -0.65597900\end{array}$

0

C

$\mathrm{C}$

C

$\mathrm{H}$

$\mathrm{H}$

$\mathrm{H}$

$\mathrm{H}$

$\mathrm{H}$

$\mathrm{H}$

$\mathrm{H}$

$\mathrm{C}$

$\mathrm{C}$

$\mathrm{H}$

$\mathrm{H}$

$\mathrm{H}$

C

$\mathrm{H}$

$\mathrm{H}$

C

$\mathrm{H}$

$\mathrm{H}$

$\mathrm{H}$

$\mathrm{H}$

$\mathrm{H}$

$\mathrm{H}$

$\mathrm{H}$

$\mathrm{H}$

$\mathrm{H}$

$\mathrm{H}$

$\mathrm{H}$

$\mathrm{H}$ $\begin{array}{lll}-1.73072300 & 0.98073100 & -1.63881700\end{array}$

$\begin{array}{lll}1.48409800 & -0.15483100 & -2.06674300\end{array}$

$\begin{array}{llll}1.29920400 & -1.63594800 & 4.18358200\end{array}$

$\begin{array}{llll}0.45359100 & -2.83141200 & 4.63704200\end{array}$

$\begin{array}{lll}2.07748200 & -1.05162100 & 5.36540600\end{array}$

$\begin{array}{llll}2.01894200 & -2.01609800 & 3.44263100\end{array}$

$\begin{array}{lll}-0.09288000 & -3.26095000 & 3.79218300\end{array}$

$\begin{array}{lll}1.08492500 & -3.61382600 & 5.07310700\end{array}$

$\begin{array}{lll}-0.27695500 & -2.51501900 & 5.38883600\end{array}$

$\begin{array}{lll}2.71244400 & -0.21483700 & 5.05569100\end{array}$

$\begin{array}{lll}1.38025400 & -0.67445200 & 6.12055700\end{array}$

$\begin{array}{lll}2.71573600 & -1.81071200 & 5.83039400\end{array}$

$\begin{array}{lll}-1.85857100 & 0.72305500 & 2.40798000\end{array}$

$\begin{array}{lll}-1.73777900 & 1.67070000 & 3.60574700\end{array}$

$\begin{array}{lll}-2.61820700 & -0.55640600 & 2.77237000\end{array}$

$\begin{array}{lll}-2.44990600 & 1.24202200 & 1.63892800\end{array}$

$\begin{array}{lll}-1.24973200 & 2.60803300 & 3.31532600\end{array}$

$\begin{array}{lll}-2.72924800 & 1.91638100 & 4.00218200\end{array}$

$\begin{array}{lll}-1.14587800 & 1.20343400 & 4.39583700\end{array}$

$-2.70386200-1.21662400 \quad 1.90295000$

$\begin{array}{lll}-2.10225300 & -1.09622300 & 3.56911900\end{array}$

$\begin{array}{lll}-3.62965700 & -0.31198100 & 3.11510100\end{array}$

$\begin{array}{lll}0.16226900 & 2.00334100 & 1.03826800\end{array}$

$\begin{array}{llll}-0.00131200 & 3.60211400 & -0.19113500\end{array}$

$\begin{array}{llll}0.55402400 & 4.00373400 & -1.54037400\end{array}$

$\begin{array}{llll}1.64585700 & 4.06094400 & -1.50927900\end{array}$

$\begin{array}{llll}0.26355000 & 3.27759800 & -2.30496900\end{array}$

$\begin{array}{llll}0.16426900 & 4.98387600 & -1.83234900\end{array}$

$\begin{array}{llll}0.54986800 & 2.33472100 & 0.18210400\end{array}$

$\begin{array}{lll}-0.91863100 & -2.04989300 & -0.68721200\end{array}$

$-1.46876200-3.33022000-0.76931100$

$\begin{array}{llll}-0.51413500 & -4.31627800 & -1.42887700\end{array}$

$\begin{array}{lll}-0.96133200 & -5.31530100 & -1.48435100\end{array}$

$\begin{array}{llll}-0.26819300 & -3.99210400 & -2.44517000\end{array}$

$0.41805700-4.38771000-0.85932500$

$\begin{array}{lll}2.51302000 & -0.22628200 & -3.00498700\end{array}$

$\begin{array}{llll}2.05456700 & 0.22689000 & -4.38503600\end{array}$

$\begin{array}{llll}2.87379600 & 0.16492400 & -5.11047100\end{array}$

$\begin{array}{lll}1.23130800 & -0.40103400 & -4.74060900\end{array}$

$\begin{array}{llll}1.70258600 & 1.26296800 & -4.35140600\end{array}$

$\begin{array}{llll}-2.77904400 & 1.47696000 & -2.41625300\end{array}$

$\begin{array}{llll}-3.83913800 & 0.41954600 & -2.69313100\end{array}$

$\begin{array}{llll}-4.26372500 & 0.04593900 & -1.75596900\end{array}$

$\begin{array}{llll}-4.65300800 & 0.83281400 & -3.29971800\end{array}$

$\begin{array}{lll}-4.40512900 & -0.42865000 & -3.23208900\end{array}$

$\begin{array}{llll}3.26068900 & -1.34727600 & 1.78676200\end{array}$

$\begin{array}{llll}0.26679900 & 4.34335300 & 0.57299700\end{array}$

$\begin{array}{llll}-1.09579200 & 3.53431800 & -0.23393300\end{array}$

$\begin{array}{lll}2.82926300 & 0.06592700 & 2.76755900\end{array}$

$\begin{array}{lll}-1.72509000 & -3.68540700 & 0.24086100\end{array}$

$\begin{array}{llll}-2.41063100 & -3.29125900 & -1.33865100\end{array}$

$\begin{array}{llll}-3.24393200 & 2.33428900 & -1.90349700\end{array}$

$\begin{array}{llll}3.36215000 & 0.39886400 & -2.68143900\end{array}$

$\begin{array}{llll}2.89041700 & -1.25978300 & -3.06668700\end{array}$

$\begin{array}{llll}-2.38406800 & 1.85951700 & -3.37087900\end{array}$

$\begin{array}{lrll}\text { TS }_{\text {3в-4в }} \text { (EtOH) } & & \\ H=-1194.241752 a u & & \\ \mathrm{~N} & -1.05153400 & 0.66771900 & 1.80618700 \\ \mathrm{C} & -0.08718900 & -0.11937700 & 2.37262800 \\ \mathrm{~N} & -0.07531900 & -0.64844900 & 3.52445000 \\ \mathrm{O} & 0.90124700 & -0.30705800 & 1.35478000 \\ \mathrm{C} & 2.29611200 & -0.10873200 & 1.67942500\end{array}$




\begin{tabular}{|c|c|c|c|}
\hline C & 2.90876800 & 0.93242400 & 0.76556600 \\
\hline $\mathrm{H}$ & 2.41397100 & 1.89845700 & 0.88985100 \\
\hline$H$ & 3.97266700 & 1.04398500 & 1.00037500 \\
\hline H & 2.81618900 & 0.63305200 & -0.28229500 \\
\hline & -0.61540900 & 0.16002900 & -0.77163300 \\
\hline 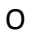 & -2.19399700 & 1.01724100 & -1.92474100 \\
\hline $\mathrm{O}$ & 1.04822300 & -0.04902800 & -2.11087800 \\
\hline 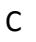 & 0.83560800 & -1.69331800 & 3.95412300 \\
\hline C & 0.01237200 & -2.94362400 & 4.28142100 \\
\hline C & 1.61183700 & -1.22431300 & 5.18724400 \\
\hline 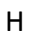 & 1.55404100 & -1.97318100 & 3.16901300 \\
\hline 1 & -0.53174600 & -3.29226600 & 3.39882300 \\
\hline 1 & 0.66003000 & -3.75563400 & 4.63008000 \\
\hline+ & -0.71824900 & -2.72043600 & 5.06566100 \\
\hline+ & 2.22671400 & -0.34532000 & 4.96754100 \\
\hline H & 0.91375000 & -0.94830600 & 5.98409400 \\
\hline 1 & 2.26928500 & -2.01667600 & 5.56044900 \\
\hline C & -2.34750500 & 0.85416800 & 2.48258000 \\
\hline C & -2.22118800 & 1.63753600 & 3.79245800 \\
\hline C & -3.11568700 & -0.45579500 & 2.66792100 \\
\hline 1 & -2.92545100 & 1.47597600 & 1.78298100 \\
\hline H & -1.71034200 & 2.59219900 & 3.62747800 \\
\hline - & -3.21363400 & 1.85160300 & 4.20406100 \\
\hline - & -1.65157500 & 1.06092300 & 4.52432200 \\
\hline H & -3.20048400 & -0.99339800 & 1.71793300 \\
\hline H & -2.60889000 & -1.10096600 & 3.38875900 \\
\hline-1 & -4.12664600 & -0.25097600 & 3.03590100 \\
\hline $\mathrm{H}$ & -0.57959900 & 1.76307500 & 1.18955500 \\
\hline C & -0.56021100 & 3.69942400 & 0.09571600 \\
\hline C & -0.04926400 & 4.24348600 & -1.22653700 \\
\hline H & 1.04084200 & 4.16442100 & -1.28096400 \\
\hline H & -0.47870400 & 3.68485400 & -2.06412200 \\
\hline - & -0.32437500 & 5.29732800 & -1.34274900 \\
\hline $\mathrm{O}$ & -0.18070600 & 2.34550700 & 0.25982100 \\
\hline $\mathrm{O}$ & -1.37447500 & -1.83301200 & -0.60737200 \\
\hline C & -1.97216400 & -3.08333200 & -0.44520900 \\
\hline C & -1.06880200 & -4.20792500 & -0.93349200 \\
\hline $\mathrm{H}$ & -1.55075500 & -5.18303100 & -0.79905700 \\
\hline $\mathrm{H}$ & -0.83826300 & -4.08112500 & -1.99608300 \\
\hline H & -0.12525200 & -4.21041100 & -0.37837500 \\
\hline C & 2.02861800 & -0.42520100 & -3.03131100 \\
\hline C & 1.62235300 & -0.09425700 & -4.46085800 \\
\hline - & 2.40692400 & -0.38744000 & -5.16770500 \\
\hline H & 0.70202800 & -0.62217700 & -4.73091800 \\
\hline $\mathrm{H}$ & 1.44372800 & 0.98011900 & -4.56990400 \\
\hline C & -3.21498800 & 1.44354000 & -2.77619400 \\
\hline C & -4.28678100 & 0.37784300 & -2.96125900 \\
\hline H & -4.74256100 & 0.11906500 & -2.00021400 \\
\hline $\mathrm{H}$ & -5.07682200 & 0.73210300 & -3.63313200 \\
\hline H & -3.85361600 & -0.53224800 & -3.38818900 \\
\hline $\mathrm{H}$ & 2.80065300 & -1.07498500 & 1.57292800 \\
\hline $\mathrm{H}$ & -0.14882900 & 4.29097800 & 0.92677600 \\
\hline H & -1.65557200 & 3.79928200 & 0.13832500 \\
\hline $\mathrm{H}$ & 2.35972900 & 0.19896200 & 2.72625600 \\
\hline 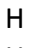 & -2.21709000 & -3.24418100 & 0.61656500 \\
\hline $\mathrm{H}$ & -2.92555400 & -3.11153800 & -0.99634400 \\
\hline H & -3.67343000 & 2.35915100 & -2.37037600 \\
\hline $\mathrm{H}$ & 2.97489900 & 0.08785900 & -2.79342500 \\
\hline & 2.22727500 & -1.50552900 & -2.94632100 \\
\hline & -2.79116100 & 1.71249200 & -3.75658600 \\
\hline
\end{tabular}

Int-4B (EtOH)

$H=-1194.251345 a u$ $\begin{array}{lll}-0.54569400 & 0.86422100 & 2.49387200\end{array}$ $\begin{array}{lll}0.41589400 & -0.09064200 & 2.46462400\end{array}$ $\begin{array}{lll}0.63648500 & -0.94975400 & 3.37292400\end{array}$ $\begin{array}{lll}1.11361600 & -0.01066800 & 1.23017200\end{array}$ $\begin{array}{lll}2.48505100 & 0.46469100 & 1.32414300\end{array}$ $\begin{array}{lll}2.56937700 & 1.90767400 & 1.77904300\end{array}$ $\begin{array}{lll}2.20290100 & 2.02983100 & 2.80235000\end{array}$ $\begin{array}{lll}3.61509800 & 2.23170900 & 1.75494000\end{array}$ $\begin{array}{lll}1.98694500 & 2.55735600 & 1.12052200\end{array}$ $\begin{array}{lll}-0.20702900 & 0.32347500 & -1.07814400\end{array}$ $\begin{array}{llll}-1.19520200 & 0.81562600 & -2.90243000\end{array}$ $\begin{array}{llll}1.79844600 & 0.03343600 & -1.83334100\end{array}$ $\begin{array}{llll}1.54507700 & -2.06917200 & 3.20243000\end{array}$ $\begin{array}{llll}0.75085400 & -3.36987400 & 3.35449600\end{array}$ $\begin{array}{llll}2.65440400 & -1.98937200 & 4.25498400\end{array}$ $\begin{array}{llll}2.00385200 & -2.07819500 & 2.20137500\end{array}$ $\begin{array}{lll}-0.03245600 & -3.43690800 & 2.59404100\end{array}$ $\begin{array}{lll}1.40663100 & -4.24148200 & 3.25136000\end{array}$ $\begin{array}{llll}0.27316100 & -3.40934900 & 4.33906800\end{array}$ $3.23906600-1.06909900 \quad 4.15249200$ $\begin{array}{llll}2.21796300 & -1.99308200 & 5.25911100\end{array}$ $\begin{array}{llll}3.33779500 & -2.84113800 & 4.17076300\end{array}$ $\begin{array}{lll}-1.53898300 & 1.02448400 & 3.55605700\end{array}$ $\begin{array}{lll}-0.89657900 & 1.42402200 & 4.88757500\end{array}$ $\begin{array}{lll}-2.43970400 & -0.20438000 & 3.69691100\end{array}$ $\begin{array}{lll}-2.16161900 & 1.86024700 & 3.21364500\end{array}$ $\begin{array}{lll}-0.30710800 & 2.33970300 & 4.77538300\end{array}$ $\begin{array}{lll}-1.66854300 & 1.60639500 & 5.64255100\end{array}$ $\begin{array}{lll}-0.24000600 & 0.62606700 & 5.24231100\end{array}$ $\begin{array}{lll}-2.91533600 & -0.44603200 & 2.74172900\end{array}$ $\begin{array}{lll}-1.85772400 & -1.06783200 & 4.02624900\end{array}$ $\begin{array}{lll}-3.22614400 & -0.01056900 & 4.43379100\end{array}$ $\begin{array}{lll}-0.52834600 & 1.54778700 & 1.73282200\end{array}$ $\begin{array}{llll}-0.94657000 & 3.58510400 & -0.17889300\end{array}$ $\begin{array}{llll}-0.31914200 & 4.23925900 & -1.40315500\end{array}$ $\begin{array}{llll}0.77349400 & 4.23791900 & -1.32449900\end{array}$ $\begin{array}{llll}-0.59776800 & 3.69524600 & -2.31202300\end{array}$ $\begin{array}{llll}-0.65178600 & 5.27792300 & -1.51050600\end{array}$ $\begin{array}{llll}-0.54236800 & 2.24968900 & -0.02136000\end{array}$ $\begin{array}{llll}-1.34779800 & -1.39406500 & -0.48179900\end{array}$ $\begin{array}{llll}-2.15053000 & -2.49479600 & -0.18070400\end{array}$ $\begin{array}{lll}-1.62275600 & -3.77570300 & -0.81390500\end{array}$ $\begin{array}{lll}-2.26357300 & -4.62923300 & -0.56481600\end{array}$ $\begin{array}{llll}-1.58817700 & -3.67954300 & -1.90410500\end{array}$ $\begin{array}{lll}-0.60997700 & -3.99137900 & -0.45824600\end{array}$ $\begin{array}{llll}2.88830000 & -0.31781900 & -2.63223100\end{array}$ $2.67384700-1.64357500-3.35097100$ $\begin{array}{llll}3.54395500 & -1.90477800 & -3.96396200\end{array}$ $\begin{array}{llll}2.50887400 & -2.45015300 & -2.62881300\end{array}$ $\begin{array}{llll}1.79863200 & -1.58756000 & -4.00700900\end{array}$ $\begin{array}{llll}-1.83815300 & 1.05842700 & -4.11718900\end{array}$ $\begin{array}{llll}-3.13853300 & 0.27583600 & -4.23997600\end{array}$ $\begin{array}{llll}-3.82790800 & 0.54766100 & -3.43421400\end{array}$ $\begin{array}{llll}-3.62957700 & 0.48183400 & -5.19784700\end{array}$ $\begin{array}{lll}-2.94767300 & -0.80007000 & -4.17520100\end{array}$ $\begin{array}{llll}2.86578000 & 0.34009100 & 0.30851300\end{array}$ $\begin{array}{lll}-0.67028900 & 4.15812200 & 0.72113700\end{array}$ $\begin{array}{llll}-2.04429600 & 3.64246600 & -0.25893700\end{array}$ $\begin{array}{llll}3.03626600 & -0.19888600 & 1.99540500\end{array}$ $\begin{array}{lll}-2.20842000 & -2.62256400 & 0.91167200\end{array}$ $\begin{array}{llll}-3.17916700 & -2.31158300 & -0.53040300\end{array}$ $\begin{array}{llll}-2.04719500 & 2.13567900 & -4.21375000\end{array}$ $\begin{array}{llll}3.07883000 & 0.47461600 & -3.37349100\end{array}$ 
$\begin{array}{llll}\mathrm{H} & 3.79654300 & -0.38570700 & -2.01002800\end{array}$

H $\quad \begin{array}{lllll}H & -1.16781000 & 0.79322900 & -4.94972500\end{array}$

pre-Cat

$H=-940.840765$ au

$\begin{array}{llll}\text { Th } & 0.16242500 & 0.67607700 & 0.34585700\end{array}$

Si $\quad \begin{array}{llll}0.38779800 & 3.88082500 & 1.95052200\end{array}$

Si $\quad \begin{array}{llll}2.38794900 & 1.76694400 & 2.90891100\end{array}$

Si $\quad \begin{array}{llll}2.12583400 & 0.55442600 & 1.15247800\end{array}$

Si $\quad \begin{array}{lllll} & -2.76515600 & 1.00814500 & -1.87350400\end{array}$

Si $\quad \begin{array}{llll}1.66903700 & -1.61294800 & -1.10368900\end{array}$

Si $\quad 2.68668700 \quad 1.12234200 \quad-2.37386700$

$\begin{array}{llll}\mathrm{N} & 1.04686100 & 2.24152100 & 1.86090200\end{array}$

N $\quad \begin{array}{llll}\mathrm{N} & -2.09320500 & 0.84671600 & -0.24496800\end{array}$

N $\quad \begin{array}{lllll}1.64110500 & 0.17402700 & -1.32016100\end{array}$

$\begin{array}{llll}\text { C } & 2.40054800 & 0.74316300 & -4.21177700\end{array}$

H $\quad \begin{array}{llll}\text { H } & 1.35827600 & 0.90948900 & -4.50009600\end{array}$

H $\quad 3.02899900 \quad 1.38939900 \quad-4.83530600$

H $\quad 2.65323500-0.29468900 \quad-4.45097700$

$\begin{array}{lllll}\text { C } & 4.53379600 & 0.85882200 & -2.01758900\end{array}$

H $\quad 4.86840600 \quad-0.14156400 \quad-2.30666800$

H $\quad 5.12991700 \quad 1.58331600 \quad-2.58426000$

H $\quad 4.76213600 \quad 0.99438400 \quad-0.95579400$

$\begin{array}{lllll}\text { C } & 2.32048400 & 2.96556100 & -2.08580700\end{array}$

$\mathrm{H} \quad 1.29932000 \quad 3.22906900-2.38152900$

H $\quad 2.46310400 \quad 3.24164900 \quad-1.03487900$

H $\quad 2.99983400 \quad 3.58557200 \quad-2.68132000$

C $\quad-1.45652400 \quad 1.83536200 \quad-2.96970100$

H $\quad-0.52666700 \quad 1.25572700 \quad-2.99277400$

H $\quad-1.81721400 \quad 1.91081700 \quad-4.00149800$

H $\quad-1.22762100 \quad 2.84976100 \quad-2.62559300$

C $\quad-4.33304100 \quad 2.07746700 \quad-1.92947100$

H $\quad-4.64926900 \quad 2.21286200 \quad-2.97011000$

H $\quad-5.16716600 \quad 1.61292700 \quad-1.39352600$

H $\quad-4.16195100 \quad 3.06883900 \quad-1.49865800$

C $\quad-3.18554300 \quad-0.67704900 \quad-2.63870200$

$\mathrm{H} \quad-3.97812600-1.18763100-2.08336500$

H $\quad-3.52636600 \quad-0.55576000 \quad-3.67325300$

H $\quad-2.31132100-1.33576500 \quad-2.64923400$

$\begin{array}{llll}\text { C } & -1.96170900 & 0.12467700 & 2.61878500\end{array}$

$\begin{array}{llll}\mathrm{H} & -1.40303200 & -0.81021800 & 2.47007800\end{array}$

H $\quad \begin{array}{llll}H & -1.26662900 & 0.92451900 & 2.91321100\end{array}$

H $\quad-2.59465100 \quad-0.04116700 \quad 3.49803600$

$\begin{array}{llll}\text { C } & -4.29996500 & -0.91802100 & 0.94241900\end{array}$

H $\quad-4.83407900 \quad-1.11566200 \quad 1.87861700$

H $\quad-5.05129900-0.72416100 \quad 0.17012400$

$\begin{array}{llll}\text { H } & -3.75399100 & -1.82433700 & 0.66344900\end{array}$

$\begin{array}{lllll}\text { C } & -4.14355000 & 2.05926400 & 1.69397700\end{array}$

H $\quad-4.66110700 \quad 1.84888500 \quad 2.63691100$

$\begin{array}{lllll}H & -3.51039300 & 2.93865900 & 1.84578100\end{array}$

H $\quad-4.90139000 \quad 2.31958400 \quad 0.94967700$

$\begin{array}{llll}\text { C } & -0.63420500 & 4.21122000 & 0.37749600\end{array}$

$\begin{array}{llll}\text { H } & -1.11497000 & 5.19225800 & 0.46744000\end{array}$

$\begin{array}{lllll}\mathrm{H} & 0.00104400 & 4.24758900 & -0.51442600\end{array}$

$\mathrm{H} \quad-1.43549600 \quad 3.48210900 \quad 0.20647900$

$\begin{array}{llll}\text { C } & -0.76745600 & 4.12794400 & 3.43780100\end{array}$

$\begin{array}{lllll}\mathrm{H} & -0.23471500 & 4.01987900 & 4.38699700\end{array}$

H $\quad-1.20676400 \quad 5.13187700 \quad 3.41429800$

$\begin{array}{llll}\text { H } & -1.58995300 & 3.40569500 & 3.43411600\end{array}$

$\begin{array}{llll}\text { C } & 1.72202700 & 5.22926100 & 2.02638700\end{array}$

H $\quad 2.42008000 \quad 5.14889200 \quad 1.18750800$

$\begin{array}{llll}\mathrm{H} & 1.24998600 & 6.21721000 & 1.97670500\end{array}$

$\begin{array}{llll}H & 2.30062600 & 5.18972600 & 2.95473900\end{array}$ $\begin{array}{lll}2.53893300 & -0.13048500 & 2.85693800\end{array}$

$\begin{array}{llll}1.63886800 & -0.64547700 & 3.21037800\end{array}$

$\begin{array}{llll}2.79162500 & -0.52198300 & 1.86477700\end{array}$

$\begin{array}{lll}3.35866900 & -0.42911700 & 3.52068000\end{array}$

$\begin{array}{llll}2.13148900 & 2.24723800 & 4.72794600\end{array}$

$\begin{array}{llll}2.13215600 & 3.33226600 & 4.87358800\end{array}$

$\begin{array}{lll}1.18667000 & 1.85384700 & 5.11596200\end{array}$

$\begin{array}{llll}2.94354700 & 1.83541800 & 5.33818800\end{array}$

$\begin{array}{lll}4.05981500 & 2.46066100 & 2.34134200\end{array}$

$\begin{array}{lll}4.86788900 & 2.06830900 & 2.96935600\end{array}$

$\begin{array}{lll}4.27062500 & 2.17363200 & 1.30617900\end{array}$

$\begin{array}{lll}4.09193500 & 3.55213000 & 2.39908000\end{array}$

$\begin{array}{lll}1.00164900 & -2.52017800 & -2.62992000\end{array}$

$0.00057300-2.16629400-2.89458500$

$\begin{array}{llll}1.64753100 & -2.37706900 & -3.50234100\end{array}$

$\begin{array}{llll}0.93741300 & -3.59566000 & -2.43043000\end{array}$

$3.40416600-2.28527600-0.73059300$

$4.05314000-2.23704200-1.61047600$

$\begin{array}{lll}3.89528300 & -1.73842700 & 0.07960000\end{array}$

$3.32593700-3.33629200-0.43084500$

$\begin{array}{llll}0.52975700 & -1.76368000 & 0.40969700\end{array}$

$\begin{array}{llll}1.02503000 & -2.29807100 & 1.22619100\end{array}$

$\begin{array}{llll}-0.39837300 & -2.29748100 & 0.16129400\end{array}$

\section{dimer-C (EtOH)}

$H=-1306.854051$ au

$\begin{array}{llll}0 & -0.27241300 & 1.29830400 & 2.45451100\end{array}$

$\begin{array}{lllll}0 & -1.90536900 & 2.68054200 & 0.90007800\end{array}$

$\begin{array}{lllll}\text { C } & -2.71878600 & 3.51001100 & 0.07010500\end{array}$

$\begin{array}{lllll}\text { C } & -4.14146100 & 2.98609100 & -0.02977700\end{array}$

H $\quad-4.16277500 \quad 1.98285400 \quad-0.46885600$

$\begin{array}{llll}H & -4.60295000 & 2.94046500 & 0.96117200\end{array}$

$\begin{array}{lllll}\text { H } & -4.74964500 & 3.64276400 & -0.66171400\end{array}$

$\begin{array}{llll}\text { C } & 0.55198100 & 0.47309000 & 3.27764900\end{array}$

$\begin{array}{llll}\text { C } & 1.96602700 & 1.01801400 & 3.38832100\end{array}$

$\begin{array}{llll}\mathrm{H} & 2.42938300 & 1.08343700 & 2.39932900\end{array}$

H $\quad \begin{array}{llll}\text { H } & 1.97121500 & 2.01559600 & 3.84066400\end{array}$

H $\quad 2.58270100 \quad 0.36256600 \quad 4.01327200$

$\begin{array}{lllll}O & -1.07813600 & 1.22909500 & -1.82064700\end{array}$

$\begin{array}{lllll}0 & -2.39825300 & -0.89458400 & 0.57660900\end{array}$

$\begin{array}{lllll}0 & 0.98445500 & -0.44410800 & 0.04553100\end{array}$

$\begin{array}{lllll}0 & -3.15169400 & 4.43870000 & 3.31008500\end{array}$

$\begin{array}{lllll}0 & 0.22846200 & 4.87023000 & 2.76710900\end{array}$

$\begin{array}{llll}0 & -1.07176000 & 2.76705500 & 5.17551000\end{array}$

$\begin{array}{llll}\text { C } & -3.29169800 & -1.97009800 & 0.61944900\end{array}$

C $\quad-4.09589800-1.97799900 \quad 1.91204000$

H $\quad-4.67365200 \quad-1.05337100 \quad 2.01065100$

$\begin{array}{llll}\mathrm{H} & -4.79306500 & -2.82317600 & 1.93351000\end{array}$

$\begin{array}{llll}H & -3.43087900 & -2.05913600 & 2.77795700\end{array}$

C $\quad 2.23395700 \quad-1.02510300 \quad-0.18714400$

C $\quad 2.12706700-2.26019500-1.07113000$

H $\quad 3.11309700-2.70692600-1.24240800$

$\mathrm{H} \quad 1.48492400-3.01276200 \quad-0.60218800$

H $\quad 1.69369600 \quad-1.99999100 \quad-2.04215400$

C $\quad-1.02265700 \quad 1.62429400 \quad-3.16030400$

$\begin{array}{llll}\text { C } & -4.38957100 & 5.04024300 & 3.55178200\end{array}$

$\begin{array}{llll}\text { C } & 1.13323900 & 5.93646100 & 2.72588800\end{array}$

$\begin{array}{llll}\text { C } & 1.95182100 & 5.92630700 & 1.44244700\end{array}$

$\begin{array}{llll}\mathrm{H} & 1.29748800 & 6.00762900 & 0.56845100\end{array}$

$\begin{array}{llll}\mathrm{H} & 2.65776800 & 6.76421000 & 1.42268100\end{array}$

$\begin{array}{llll}\mathrm{H} & 2.52120600 & 4.99517400 & 1.35729700\end{array}$

$\begin{array}{lllll}\text { C } & -1.09271900 & 2.36224600 & 6.51317300\end{array}$

$\begin{array}{llll}\text { C } & -2.33649800 & 1.54726500 & 6.84320200\end{array}$ 


$\begin{array}{lrrr}\text { H } & -3.24096400 & 2.13543500 & 6.65681000 \\ \mathrm{H} & -2.38249900 & 0.64505300 & 6.22380700 \\ \mathrm{H} & -2.33779500 & 1.23952600 & 7.89497500 \\ \mathrm{H} & -2.26471100 & 3.56873300 & -0.92748400 \\ \mathrm{H} & -2.73587800 & 4.53099700 & 0.47977000 \\ \mathrm{H} & 0.09594700 & 0.39605300 & 4.27302000 \\ \mathrm{H} & 0.58716700 & -0.54268900 & 2.85640300 \\ \mathrm{H} & -0.19333900 & 1.76584600 & 6.73521200 \\ \mathrm{H} & -1.04993200 & 3.24709400 & 7.16696500 \\ \mathrm{H} & -5.05500300 & 4.33257000 & 4.07198800 \\ \mathrm{H} & 1.80869100 & 5.88359000 & 3.59400500 \\ \mathrm{H} & 0.58828400 & 6.88986900 & 2.80747700 \\ \mathrm{H} & 2.69989600 & -1.30098100 & 0.77260800 \\ \mathrm{H} & 2.90570800 & -0.29192700 & -0.66186600 \\ \mathrm{H} & 0.01702500 & 1.57302600 & -3.52068100 \\ \mathrm{H} & -1.33433600 & 2.67740400 & -3.25251400 \\ \mathrm{H} & -2.73843700 & -2.91751900 & 0.52438300 \\ \mathrm{H} & -3.97754600 & -1.91753900 & -0.24050300 \\ \mathrm{C} & -1.90889400 & 0.75608200 & -4.04293600 \\ \mathrm{H} & -1.85486200 & 1.07709100 & -5.08943300 \\ \mathrm{H} & -1.59584200 & -0.29150300 & -3.98765400 \\ \mathrm{H} & -2.95246400 & 0.81669800 & -3.71777000 \\ \mathrm{C} & -4.24891600 & 6.30785600 & 4.38358100 \\ \mathrm{H} & -5.22636500 & 6.76952300 & 4.56419700 \\ \mathrm{H} & -3.79074700 & 6.08183200 & 5.35179400 \\ \mathrm{H} & -3.61427600 & 7.03603500 & 3.86822200 \\ \mathrm{H} & -4.87817100 & 5.28304200 & 2.59437800 \\ \mathrm{Th} & -1.27828300 & 3.38941100 & 3.12353600 \\ \mathrm{Th} & -0.89561800 & 0.59352500 & 0.22951300\end{array}$

\section{Cat $_{\mathrm{C}}$ (EtOH)}

$H=-653.398001 \mathrm{au}$

$\begin{array}{llll}\text { Th } & 1.10082400 & 1.91989700 & -0.34343000 \\ \mathrm{C} & 3.74143700 & 0.73965800 & -3.55554800 \\ \mathrm{H} & 3.99582700 & 1.80422500 & -3.52654100 \\ \mathrm{H} & 4.52291800 & 0.21474100 & -4.11638500 \\ \mathrm{H} & 2.79710000 & 0.62895100 & -4.09870300 \\ \mathrm{C} & -2.33183200 & 0.78311000 & -2.72799900 \\ \mathrm{H} & -1.56925000 & 0.24828800 & -3.30369300 \\ \mathrm{H} & -3.31377400 & 0.38274000 & -3.00410800 \\ \mathrm{H} & -2.29884800 & 1.83925900 & -3.01512200 \\ \mathrm{C} & 1.48895000 & 0.04415300 & 3.52777700 \\ \mathrm{H} & 0.49789000 & -0.34209600 & 3.26765300 \\ \mathrm{H} & 2.23862200 & -0.55245300 & 2.99757900 \\ \mathrm{H} & 1.63935800 & -0.09420900 & 4.60434800 \\ \mathrm{O} & 2.62372300 & 0.84359100 & -1.41195100 \\ \mathrm{O} & 1.41545700 & 1.70870800 & 1.77323700 \\ \mathrm{O} & -0.82981100 & 1.13399300 & -0.86419300 \\ \mathrm{C} & -2.07954800 & 0.62784800 & -1.23450000 \\ \mathrm{C} & 3.61464900 & 0.18231100 & -2.14420700 \\ \mathrm{C} & 1.60675900 & 1.51309300 & 3.14450700 \\ \mathrm{O} & 1.18182900 & 4.00150700 & -0.86752300 \\ \mathrm{C} & 1.20817800 & 5.36930100 & -1.15714100 \\ \mathrm{C} & 0.25483600 & 6.15388600 & -0.26604300 \\ \mathrm{H} & -0.77376700 & 5.80376800 & -0.40175300 \\ \mathrm{H} & 0.52275400 & 6.02714900 & 0.78806800 \\ \mathrm{H} & 0.28683600 & 7.22343800 & -0.50227400 \\ \mathrm{H} & 4.58143200 & 0.27267100 & -1.62454900 \\ \mathrm{H} & 3.38340200 & -0.89330700 & -2.19246400 \\ \mathrm{H} & 2.23192500 & 5.75493500 & -1.03040300 \\ \mathrm{H} & 0.94092400 & 5.52913200 & -2.21344400 \\ \mathrm{H} & 2.59847200 & 1.89169900 & 3.43769100 \\ \mathrm{H} & 0.86653600 & 2.10136100 & 3.70916500\end{array}$

H $\quad-2.14464800-0.43642500 \quad-0.95882700$

$\begin{array}{llll}\mathrm{H} & -2.87104000 & 1.14688900 & -0.67145500\end{array}$

\section{Int-1C (EtOH)}

$H=-1037.72995 a u$

$\begin{array}{llll}\mathrm{N} & -0.27231900 & 2.18721400 & 0.45526900\end{array}$

$\begin{array}{lllll}\text { C } & & -0.68599900 & 2.33149700 & 1.61883100\end{array}$

$\begin{array}{lllll}\mathrm{N} & -0.96333400 & 2.45509400 & 2.79081900\end{array}$

$\begin{array}{llll}\mathrm{O} & 1.15502000 & -0.23370000 & 1.97459600\end{array}$

$\begin{array}{llll}\text { C } & 1.74099200 & -0.12486800 & 3.23501800\end{array}$

$\begin{array}{llll}\text { C } & 3.25898400 & -0.01610500 & 3.15484300\end{array}$

$\begin{array}{llll}\mathrm{H} & 3.55190500 & 0.87263300 & 2.58533400\end{array}$

$\begin{array}{llll}\mathrm{H} & 3.70418000 & 0.05666300 & 4.15394900\end{array}$

H $\quad 3.67894000 \quad-0.89534000 \quad 2.65508300$

Th $\quad 0.47272600 \quad-0.47238900 \quad-0.06794200$

O $\quad-1.66987600-0.83183700-0.12882500$

$\begin{array}{lllll}0 & 1.36733800 & 0.38127900 & -1.87555400\end{array}$

$\begin{array}{llll}\text { C } & -2.18101500 & 2.10369900 & 3.52470600\end{array}$

$\begin{array}{llll}\text { C } & -3.28956200 & 1.58304600 & 2.61516400\end{array}$

$\begin{array}{lllll}\text { C } & -2.61340400 & 3.30749400 & 4.35785500\end{array}$

H $\quad-1.87518700 \quad 1.30253300 \quad 4.20963500$

$\begin{array}{llll}H & -2.94137500 & 0.73984100 & 2.01076500\end{array}$

$\begin{array}{lllll}\mathrm{H} & -4.14371100 & 1.24983600 & 3.21262300\end{array}$

$\mathrm{H} \quad-3.63762600 \quad 2.37307100 \quad 1.94009200$

H $\quad-1.79404700 \quad 3.65707200 \quad 4.99129300$

$\begin{array}{llll}\mathrm{H} & -2.92469400 & 4.13392700 & 3.71064000\end{array}$

H $\quad-3.45812600 \quad 3.03691000 \quad 4.99830000$

C $\quad-0.40345600 \quad 3.29421800 \quad-0.53309900$

C $\quad-1.16346500 \quad 2.79043800 \quad-1.75446100$

$\begin{array}{lllll}\text { C } & 0.99341600 & 3.79310700 & -0.88705200\end{array}$

H $\quad-0.97138200 \quad 4.11053300 \quad-0.06660500$

H $\quad-2.15220700 \quad 2.41382100 \quad-1.47793000$

H $\quad-1.29231000 \quad 3.60323900 \quad-2.47643100$

H $\quad-0.60105300 \quad 1.98503000 \quad-2.23770000$

H $\quad \begin{array}{llll}1.51171200 & 4.17488000 & -0.00262100\end{array}$

H $\quad \begin{array}{llll}\mathrm{H} & 1.57970700 & 2.97312900 & -1.31165300\end{array}$

H $\quad 0.93036900 \quad 4.59959000 \quad-1.62487000$

$\begin{array}{lllll}0 & 1.17968200 & -2.47491400 & -0.36426000\end{array}$

C $\quad 1.68100800 \quad-3.77252200 \quad-0.46288800$

C $\quad 0.57217000 \quad-4.79585800-0.67116200$

H $\quad 0.98212500 \quad-5.80969700 \quad-0.74602200$

H $\quad-0.13463000 \quad-4.77022700 \quad 0.16446600$

H $\quad 0.01898100 \quad-4.57948200 \quad-1.59085000$

C $\quad 2.10772800 \quad 0.56854600 \quad-3.04339500$

$\begin{array}{lllll}\text { C } & 3.60562900 & 0.41529900 & -2.80721200\end{array}$

$\mathrm{H} \quad 4.16993800 \quad 0.56889900 \quad-3.73433800$

$\begin{array}{lllll}\mathrm{H} & 3.95678900 & 1.14433700 & -2.06879300\end{array}$

H $\quad 3.83284700 \quad-0.58720600 \quad-2.42987500$

C $\quad-2.93227900-1.35257600 \quad-0.41162000$

C $\quad-3.08230500 \quad-1.74051900-1.87787400$

$\mathrm{H} \quad-2.92529900-0.86994400 \quad-2.52375100$

H $\quad-2.34522200-2.50350500 \quad-2.14915200$

H $\quad-4.08174300 \quad-2.14239000 \quad-2.08109300$

H $\quad 1.47259600 \quad-1.00104400 \quad 3.84790500$

$\begin{array}{llll}\mathrm{H} & 1.33874800 & 0.75880600 & 3.75641800\end{array}$

$\begin{array}{llll}H & 2.24497900 & -4.02517700 & 0.44954200\end{array}$

H $\quad 1.90780000 \quad 1.57205500 \quad-3.45701200$

H $\quad 1.78679700-0.15163900-3.81372200$

H $\quad 2.39499400 \quad-3.83252900 \quad-1.30019400$

$\begin{array}{lllll}H & -3.12577400 & -2.23565200 & 0.21936900\end{array}$

H $\quad-3.70810400 \quad-0.61204100 \quad-0.15304500$ 


\begin{tabular}{|c|c|c|c|}
\hline \multicolumn{4}{|c|}{$\mathrm{TS}_{1 \mathrm{C}-2 \mathrm{C}}(\mathrm{EtOH})$} \\
\hline \multicolumn{4}{|c|}{$H=-1037.715573 a u$} \\
\hline $\mathrm{N}$ & -1.00532900 & 2.39597400 & 0.68306100 \\
\hline $\mathrm{C}$ & -1.15051200 & 2.40789200 & 1.96138600 \\
\hline $\mathrm{N}$ & -1.38761000 & 3.03898300 & 2.98911900 \\
\hline 0 & -0.64415400 & 0.57581000 & 2.27479600 \\
\hline C & 0.19375700 & 0.39214900 & 3.39418600 \\
\hline $\mathrm{C}$ & 1.67175300 & 0.37874000 & 3.02202600 \\
\hline $\mathrm{H}$ & 1.96644900 & 1.33639700 & 2.57782200 \\
\hline $\mathrm{H}$ & 2.29935500 & 0.21406700 & 3.90487600 \\
\hline $\mathrm{H}$ & 1.88439800 & -0.42410000 & 2.30647500 \\
\hline Th & -0.47101100 & -0.03009500 & -0.00161000 \\
\hline 0 & -2.40323500 & -0.61492000 & -0.71715700 \\
\hline 0 & 0.74020200 & 0.59122900 & -1.67040200 \\
\hline C & -2.23263400 & 2.71536200 & 4.12805300 \\
\hline C & -3.67645800 & 3.13103500 & 3.83379800 \\
\hline C & -1.69337000 & 3.41067900 & 5.37530200 \\
\hline $\mathrm{H}$ & -2.21450100 & 1.62787100 & 4.28467500 \\
\hline $\mathrm{H}$ & -4.06171200 & 2.60798100 & 2.95339600 \\
\hline $\mathrm{H}$ & -4.32478700 & 2.89315100 & 4.68389100 \\
\hline $\mathrm{H}$ & -3.73403800 & 4.20827500 & 3.64702500 \\
\hline $\mathrm{H}$ & -0.66591100 & 3.10011900 & 5.58654400 \\
\hline $\mathrm{H}$ & -1.69508200 & 4.49676800 & 5.23722800 \\
\hline $\mathrm{H}$ & -2.31184200 & 3.17182900 & 6.24619300 \\
\hline C & -1.48138400 & 3.59274500 & -0.04424800 \\
\hline C & -1.92033700 & 3.17533800 & -1.44368100 \\
\hline C & -0.37741600 & 4.64850600 & -0.07881200 \\
\hline $\mathrm{H}$ & -2.34485800 & 4.01005300 & 0.49267300 \\
\hline $\mathrm{H}$ & -2.73884300 & 2.44963500 & -1.39956100 \\
\hline $\mathrm{H}$ & -2.26466800 & 4.04251000 & -2.01595300 \\
\hline $\mathrm{H}$ & -1.08470600 & 2.71978900 & -1.98771000 \\
\hline $\mathrm{H}$ & -0.08574300 & 4.93411500 & 0.93598900 \\
\hline $\mathrm{H}$ & 0.50565700 & 4.26037000 & -0.59730700 \\
\hline $\mathrm{H}$ & -0.71791800 & 5.54769300 & -0.60380600 \\
\hline 0 & 0.53693300 & -1.87208100 & 0.47566000 \\
\hline $\mathrm{C}$ & 1.21788300 & -3.06070700 & 0.75040600 \\
\hline $\mathrm{C}$ & 0.28478500 & -4.26387800 & 0.74287200 \\
\hline $\mathrm{H}$ & 0.83331800 & -5.18689100 & 0.96262600 \\
\hline $\mathrm{H}$ & -0.50241300 & -4.14366400 & 1.49418600 \\
\hline $\mathrm{H}$ & -0.19307100 & -4.37282300 & -0.23599700 \\
\hline $\mathrm{C}$ & 1.64229100 & 0.91464700 & -2.68685000 \\
\hline $\mathrm{C}$ & 3.08269100 & 0.92533800 & -2.19196800 \\
\hline $\mathrm{H}$ & 3.77533900 & 1.17717500 & -3.00306400 \\
\hline $\mathrm{H}$ & 3.21025700 & 1.66313700 & -1.39262400 \\
\hline $\mathrm{H}$ & 3.35938400 & -0.05767500 & -1.79719900 \\
\hline C & -3.57423500 & -1.16897500 & -1.24313100 \\
\hline $\mathrm{C}$ & -3.31583700 & -1.93201000 & -2.53506600 \\
\hline $\mathrm{H}$ & -2.88772300 & -1.26961900 & -3.29451200 \\
\hline $\mathrm{H}$ & -2.61225000 & -2.75308200 & -2.36257300 \\
\hline $\mathrm{H}$ & -4.24531400 & -2.35523200 & -2.93259300 \\
\hline $\mathrm{H}$ & -0.08132600 & -0.54866800 & 3.89250700 \\
\hline $\mathrm{H}$ & 0.01311800 & 1.20406100 & 4.11220100 \\
\hline $\mathrm{H}$ & 1.71240000 & -2.98809100 & 1.73272600 \\
\hline $\mathrm{H}$ & 1.39125100 & 1.90274400 & -3.10503600 \\
\hline $\mathrm{H}$ & 1.54369500 & 0.19216200 & -3.51216400 \\
\hline $\mathrm{H}$ & 2.01736800 & -3.20991000 & 0.00741300 \\
\hline $\mathrm{H}$ & -4.02952900 & -1.84366300 & -0.50132800 \\
\hline $\mathrm{H}$ & -4.30603600 & -0.36744900 & -1.42945700 \\
\hline
\end{tabular}

\section{Int-2C (EtOH)}

$\mathrm{H}=-1037.727735 \mathrm{au}$

N $\quad-0.34875200 \quad 1.74692200 \quad 0.25011500$ $\begin{array}{lll}-0.65325100 & 1.55014200 & 1.55014100\end{array}$

$\begin{array}{lll}-1.10218600 & 2.38377200 & 2.39841000\end{array}$

$\begin{array}{lll}-0.43542100 & 0.13739800 & 1.79779100\end{array}$

$\begin{array}{lll}0.49265300 & -0.20366600 & 2.85127100\end{array}$

$\begin{array}{lll}1.89147200 & 0.32662500 & 2.60106800\end{array}$

$\begin{array}{lll}1.89378300 & 1.41728200 & 2.52159900\end{array}$

$\begin{array}{llll}2.54373100 & 0.04655000 & 3.43471100\end{array}$

$\begin{array}{lll}2.31825100 & -0.10249800 & 1.68845900\end{array}$

$\begin{array}{llll}0.05118500 & -0.44382700 & -0.72775300\end{array}$

$-1.82552400-1.19624000-1.41406000$

$\begin{array}{llll}1.21804000 & 0.02077400 & -2.45871000\end{array}$

$\begin{array}{lll}-1.69581000 & 2.03313000 & 3.67361000\end{array}$

$\begin{array}{lll}-3.13755300 & 2.55293400 & 3.68712300\end{array}$

$\begin{array}{lll}-0.88918200 & 2.65945500 & 4.81476000\end{array}$

$\begin{array}{lll}-1.74507600 & 0.94274900 & 3.82697300\end{array}$

$\begin{array}{lll}-3.72023600 & 2.09372800 & 2.88297100\end{array}$

$\begin{array}{lll}-3.62817500 & 2.32879500 & 4.64115100\end{array}$

$\begin{array}{lll}-3.14775100 & 3.63698600 & 3.53419000\end{array}$

$\begin{array}{lll}0.13811000 & 2.28049500 & 4.83634300\end{array}$

$\begin{array}{lll}-0.83714400 & 3.74453400 & 4.67862300\end{array}$

$\begin{array}{lll}-1.35129400 & 2.45291500 & 5.78626700\end{array}$

$\begin{array}{llll}-0.62743300 & 3.07332400 & -0.29758300\end{array}$

$\begin{array}{lll}-0.88378400 & 2.95727700 & -1.79916700\end{array}$

$\begin{array}{llll}0.51640600 & 4.04332100 & 0.00646000\end{array}$

$\begin{array}{lll}-1.53242600 & 3.46007100 & 0.19218200\end{array}$

$\begin{array}{lll}-1.74888900 & 2.31451800 & -2.00321700\end{array}$

$\begin{array}{llll}-1.09056600 & 3.93826100 & -2.23891400\end{array}$

$\begin{array}{lll}-0.01127700 & 2.54077700 & -2.31844200\end{array}$

$\begin{array}{llll}0.66283100 & 4.11953700 & 1.08701000\end{array}$

$\begin{array}{llll}1.44911400 & 3.69570600 & -0.45303700\end{array}$

$\begin{array}{llll}0.29663400 & 5.04498900 & -0.38032200\end{array}$

$\begin{array}{lll}1.19848200 & -2.04255500 & 0.15189200\end{array}$

$\begin{array}{llll}1.92201500 & -3.10172700 & 0.70660100\end{array}$

$\begin{array}{llll}1.08342900 & -4.36769700 & 0.81979200\end{array}$

$\begin{array}{lll}1.66622100 & -5.18612100 & 1.25721000\end{array}$

$\begin{array}{llll}0.20614000 & -4.19494500 & 1.45194700\end{array}$

$\begin{array}{llll}0.73274500 & -4.68541100 & -0.16732900\end{array}$

$\begin{array}{llll}2.05605500 & 0.22243900 & -3.56184500\end{array}$

$\begin{array}{lll}3.52679000 & 0.17771100 & -3.17186400\end{array}$

$\begin{array}{llll}4.16810700 & 0.33874800 & -4.04558400\end{array}$

$\begin{array}{llll}3.75275700 & 0.95442700 & -2.43386100\end{array}$

$\begin{array}{lll}3.77946100 & -0.79371200 & -2.73474500\end{array}$

$\begin{array}{llll}-2.99160500 & -1.75094200 & -1.95610900\end{array}$

$-2.70707200-2.54558200-3.22261100$

$\begin{array}{lll}-2.25648100 & -1.90405400 & -3.98691800\end{array}$

$\begin{array}{llll}-2.01442400 & -3.36774800 & -3.01435000\end{array}$

$\begin{array}{llll}-3.63003300 & -2.97095700 & -3.63229100\end{array}$

$\begin{array}{lll}0.48312700 & -1.29580300 & 2.87694200\end{array}$

$\begin{array}{lll}0.10147800 & 0.16569600 & 3.80155300\end{array}$

$\begin{array}{llll}2.29426100 & -2.81854300 & 1.70534700\end{array}$

$\begin{array}{llll}1.82510400 & 1.19488300 & -4.02310500\end{array}$

$\begin{array}{lll}1.85132600 & -0.54486900 & -4.32376200\end{array}$

$\begin{array}{llll}2.81141600 & -3.30358100 & 0.08954300\end{array}$

$\begin{array}{llll}-3.46692000 & -2.40332500 & -1.20795500\end{array}$

$-3.70985500-0.94689800-2.17670700$

\section{Int-3C (EtOH)}

$H=-1192.648925$ au

$\begin{array}{llll}\mathrm{N} & -0.55858800 & 0.48019100 & 1.77622200\end{array}$

C $\quad 0.37958500 \quad-0.23657700 \quad 2.44220400$

N $\quad 0.40246900-0.62200600 \quad 3.65305100$

$\begin{array}{llll}\mathrm{O} & 1.38913700 & -0.55659800 & 1.45187200\end{array}$

C $\quad 2.77829600 \quad-0.35867300 \quad 1.78885200$ 


\begin{tabular}{|c|c|c|c|}
\hline C & 3.45833800 & 0.52525100 & 0.76272000 \\
\hline $\mathrm{H}$ & 2.99018400 & 1.51262800 & 0.72454300 \\
\hline $\mathrm{H}$ & 4.51356700 & 0.64822300 & 1.02916800 \\
\hline $\mathrm{H}$ & 3.40856500 & 0.08188600 & -0.23581400 \\
\hline Th & -0.12152500 & -0.06959700 & -0.67657900 \\
\hline $\mathrm{O}$ & -1.71902000 & 0.94861100 & -1.68456900 \\
\hline 0 & 1.49599100 & -0.17164900 & -2.10183600 \\
\hline C & 1.28382200 & -1.63387900 & 4.20091600 \\
\hline C & 0.43239700 & -2.82657100 & 4.65093000 \\
\hline C & 2.06460500 & -1.05666500 & 5.38463000 \\
\hline $\mathrm{H}$ & 2.00215700 & -2.01561300 & 3.45937400 \\
\hline $\mathrm{H}$ & -0.11538300 & -3.25158300 & 3.80461100 \\
\hline $\mathrm{H}$ & 1.05961200 & -3.61299200 & 5.08579100 \\
\hline $\mathrm{H}$ & -0.29738300 & -2.50848800 & 5.40277000 \\
\hline $\mathrm{H}$ & 2.70395600 & -0.22246100 & 5.07707700 \\
\hline $\mathrm{H}$ & 1.36907900 & -0.67776900 & 6.14050500 \\
\hline $\mathrm{H}$ & 2.69881400 & -1.82015100 & 5.84802800 \\
\hline C & -1.85366100 & 0.74900200 & 2.42387600 \\
\hline $\mathrm{C}$ & -1.72704900 & 1.69592200 & 3.62166300 \\
\hline C & -2.62467700 & -0.52388300 & 2.78755700 \\
\hline $\mathrm{H}$ & -2.44080500 & 1.27266500 & 1.65423800 \\
\hline $\mathrm{H}$ & -1.23087900 & 2.62907400 & 3.33154000 \\
\hline $\mathrm{H}$ & -2.71675600 & 1.94996400 & 4.01734000 \\
\hline $\mathrm{H}$ & -1.13951500 & 1.22387400 & 4.41221500 \\
\hline $\mathrm{H}$ & -2.71556700 & -1.18299700 & 1.91761700 \\
\hline $\mathrm{H}$ & -2.11313000 & -1.06856700 & 3.58383400 \\
\hline $\mathrm{H}$ & -3.63426400 & -0.27165100 & 3.13018200 \\
\hline $\mathrm{H}$ & 0.16331500 & 1.98694500 & 1.05046900 \\
\hline $\mathrm{C}$ & 0.01141900 & 3.62362700 & -0.13589000 \\
\hline C & 0.52405900 & 4.03515800 & -1.49922100 \\
\hline $\mathrm{H}$ & 1.61725700 & 4.07112500 & -1.50785900 \\
\hline $\mathrm{H}$ & 0.19122700 & 3.32653900 & -2.26324300 \\
\hline $\mathrm{H}$ & 0.14352000 & 5.02696700 & -1.76245600 \\
\hline 0 & 0.54926000 & 2.34087400 & 0.19823700 \\
\hline 0 & -0.88539000 & -2.06254000 & -0.71088100 \\
\hline C & -1.42475300 & -3.34943900 & -0.79994500 \\
\hline $\mathrm{C}$ & -0.45709100 & -4.32505300 & -1.45549200 \\
\hline $\mathrm{H}$ & -0.89439100 & -5.32812500 & -1.51629300 \\
\hline $\mathrm{H}$ & -0.20752800 & -3.99638200 & -2.46948500 \\
\hline $\mathrm{H}$ & 0.47203200 & -4.38932400 & -0.88003000 \\
\hline C & 2.49831300 & -0.26988200 & -3.06976000 \\
\hline $\mathrm{C}$ & 2.02090700 & 0.21649200 & -4.43165400 \\
\hline $\mathrm{H}$ & 2.81801700 & 0.13461100 & -5.17936400 \\
\hline $\mathrm{H}$ & 1.16672200 & -0.37719500 & -4.77294300 \\
\hline $\mathrm{H}$ & 1.70724700 & 1.26394500 & -4.37678700 \\
\hline C & -2.76910000 & 1.45382000 & -2.45721200 \\
\hline C & -3.85040100 & 0.41084700 & -2.70440400 \\
\hline $\mathrm{H}$ & -4.27084000 & 0.06046400 & -1.75642400 \\
\hline $\mathrm{H}$ & -4.66398300 & 0.82747500 & -3.30904800 \\
\hline $\mathrm{H}$ & -3.43682800 & -0.45374200 & -3.23325100 \\
\hline $\mathrm{H}$ & 3.25727500 & -1.34291200 & 1.83563400 \\
\hline $\mathrm{H}$ & 0.32146700 & 4.34860700 & 0.62810700 \\
\hline $\mathrm{H}$ & -1.08535100 & 3.57940100 & -0.13809200 \\
\hline $\mathrm{H}$ & 2.82405600 & 0.09114000 & 2.78426400 \\
\hline $\mathrm{H}$ & -1.68425600 & -3.70856300 & 0.20772700 \\
\hline $\mathrm{H}$ & -2.36290600 & -3.31636600 & -1.37525300 \\
\hline $\mathrm{H}$ & -3.21174000 & 2.32677800 & -1.95166000 \\
\hline $\mathrm{H}$ & 3.37679500 & 0.31989900 & -2.75995400 \\
\hline 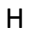 & 2.83506600 & -1.31557900 & -3.15150600 \\
\hline 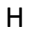 & -2.37888400 & 1.81445700 & -3.42202700 \\
\hline
\end{tabular}

$\mathrm{TS}_{3 \mathrm{C}-4 \mathrm{C}}$ (EtOH)

$\mathrm{H}=-1192.650271 \mathrm{au}$ $\begin{array}{lll}-1.05138300 & 0.67095500 & 1.80696300\end{array}$

$\begin{array}{lll}-0.10150400 & -0.11420200 & 2.39486400\end{array}$

$\begin{array}{lll}-0.10215900 & -0.62519100 & 3.55562400\end{array}$

$\begin{array}{llll}0.89744000 & -0.33209700 & 1.38937600\end{array}$

$\begin{array}{lll}2.28862700 & -0.13345300 & 1.72584300\end{array}$

$\begin{array}{llll}2.91864600 & 0.87376700 & 0.78568300\end{array}$

$\begin{array}{llll}2.42766600 & 1.84605300 & 0.87200100\end{array}$

$\begin{array}{lll}3.98032200 & 0.98841100 & 1.02866900\end{array}$

$\begin{array}{llll}2.83863500 & 0.54045100 & -0.25335800\end{array}$

$\begin{array}{llll}-0.58589700 & 0.10799600 & -0.79255500\end{array}$

$\begin{array}{llll}-2.17630500 & 0.92881500 & -1.96246300\end{array}$

$\begin{array}{llll}1.07549200 & -0.08638500 & -2.14482400\end{array}$

$\begin{array}{llll}0.79279400 & -1.67513400 & 4.00515300\end{array}$

$\begin{array}{lll}-0.04710800 & -2.91226100 & 4.34053600\end{array}$

$\begin{array}{llll}1.56501600 & -1.20082000 & 5.23881300\end{array}$

$\begin{array}{llll}1.51438400 & -1.97271200 & 3.22941700\end{array}$

$\begin{array}{lll}-0.58819500 & -3.26498500 & 3.45766600\end{array}$

$\begin{array}{lll}0.58816900 & -3.72754500 & 4.70413200\end{array}$

$\begin{array}{llll}-0.78144500 & -2.67124600 & 5.11600100\end{array}$

$\begin{array}{llll}2.19174200 & -0.33169900 & 5.01340000\end{array}$

$\begin{array}{lll}0.86417500 & -0.90726800 & 6.02693800\end{array}$

$\begin{array}{llll}2.21036800 & -1.99620900 & 5.62654100\end{array}$

$\begin{array}{lll}-2.34855700 & 0.88327500 & 2.47201500\end{array}$

$\begin{array}{lll}-2.22375200 & 1.68649100 & 3.77001900\end{array}$

$\begin{array}{lll}-3.13227100 & -0.41531600 & 2.67370100\end{array}$

$\begin{array}{lll}-2.91563100 & 1.49941000 & 1.75845900\end{array}$

$\begin{array}{lll}-1.70108300 & 2.63260500 & 3.59310200\end{array}$

$\begin{array}{lll}-3.21658000 & 1.91822800 & 4.17112400\end{array}$

$\begin{array}{lll}-1.66543500 & 1.11576800 & 4.51515200\end{array}$

$\begin{array}{lll}-3.21602500 & -0.96866600 & 1.73238400\end{array}$

$\begin{array}{llll}-2.63740700 & -1.05362500 & 3.40875500\end{array}$

$\begin{array}{lll}-4.14395300 & -0.19440900 & 3.03030500\end{array}$

$\begin{array}{lll}-0.56795800 & 1.75935600 & 1.16235600\end{array}$

$\begin{array}{llll}-0.50080000 & 3.70010300 & 0.09951600\end{array}$

$\begin{array}{llll}-0.03305800 & 4.23476200 & -1.24244500\end{array}$

$\begin{array}{llll}1.04909400 & 4.11255600 & -1.35084000\end{array}$

$\begin{array}{llll}-0.52467500 & 3.70055200 & -2.06171800\end{array}$

$\begin{array}{llll}-0.27009900 & 5.29977800 & -1.33842900\end{array}$

$\begin{array}{lll}-0.17566900 & 2.32932900 & 0.22992000\end{array}$

$\begin{array}{llll}-1.32198100 & -1.89738100 & -0.64798200\end{array}$

$-1.90372700-3.16465600-0.55348500$

$\begin{array}{lll}-0.97273100 & -4.25315000 & -1.06984800\end{array}$

$\begin{array}{lll}-1.44211100 & -5.24024600 & -0.98942400\end{array}$

$-0.72075800-4.07619700-2.12037900$

$\begin{array}{lll}-0.04171100 & -4.26664800 & -0.49408900\end{array}$

$\begin{array}{llll}2.05677000 & -0.39206300 & -3.09266100\end{array}$

$\begin{array}{llll}1.61745900 & -0.02529300 & -4.50344500\end{array}$

$\begin{array}{llll}2.40051100 & -0.26502700 & -5.23176300\end{array}$

$\begin{array}{llll}0.71202700 & -0.57549500 & -4.77939500\end{array}$

$\begin{array}{llll}1.39968700 & 1.04530400 & -4.57132900\end{array}$

$\begin{array}{llll}-3.20653700 & 1.35393800 & -2.80654700\end{array}$

$-4.40817500 \quad 0.42041300-2.75423100$

$\begin{array}{llll}-4.81167300 & 0.36853400 & -1.73785600\end{array}$

$\begin{array}{llll}-5.20334700 & 0.76980700 & -3.42257600\end{array}$

$\begin{array}{lll}-4.12196500 & -0.59131300 & -3.05836800\end{array}$

$\begin{array}{llll}2.78942900 & -1.10517800 & 1.65714400\end{array}$

$\begin{array}{lll}-0.02396500 & 4.26541300 & 0.91387100\end{array}$

$\begin{array}{lll}-1.58737300 & 3.84560800 & 0.19755000\end{array}$

$\begin{array}{llll}2.34163000 & 0.20747800 & 2.76303900\end{array}$

$\begin{array}{lll}-2.16754400 & -3.37312000 & 0.49506400\end{array}$

$\begin{array}{llll}-2.84485000 & -3.18231500 & -1.12521700\end{array}$

$\begin{array}{llll}-3.51853000 & 2.37106900 & -2.52189200\end{array}$

$\begin{array}{llll}2.98694000 & 0.14632700 & -2.84849800\end{array}$ 
H $\quad 2.29486900-1.46664600 \quad-3.04891100$

$\begin{array}{lllll}H & -2.83391800 & 1.41695600 & -3.84076600\end{array}$

\section{Int-4C (EtOH)}

$\mathrm{H}=-1192.657775 \mathrm{au}$

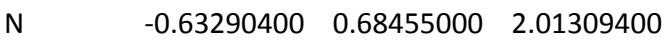

$\begin{array}{llll}\text { C } & 0.37936600 & -0.15007700 & 2.45721600\end{array}$

N $\quad \begin{array}{llll}0.35868900 & -0.82118400 & 3.52666600\end{array}$

$\begin{array}{lllll}\text { O } & 1.34719400 & -0.17229100 & 1.44333600\end{array}$

$\begin{array}{lllll}\text { C } & 2.74088500 & 0.02026100 & 1.78700800\end{array}$

$\begin{array}{lllll}\text { C } & 3.34150400 & 1.06976300 & 0.87667400\end{array}$

$\begin{array}{llll}\mathrm{H} & 2.82474500 & 2.02582200 & 0.98920900\end{array}$

$\begin{array}{llll}H & 4.39978000 & 1.20554500 & 1.12266400\end{array}$

H $\quad 3.26646600 \quad 0.76031000 \quad-0.16994600$

Th $\quad-0.10605000 \quad 0.27242600 \quad-0.86189700$

$\begin{array}{lllll}0 & -1.64924700 & 0.79032400 & -2.25093000\end{array}$

$\begin{array}{lllll}0 & 1.63535500 & -0.02982900 & -2.09352200\end{array}$

C $\quad 1.27956800 \quad-1.90452300 \quad 3.82988100$

$\begin{array}{lllll}\text { C } & 0.46673100 & -3.19091300 & 3.99860600\end{array}$

$\begin{array}{llll}\text { C } & 2.04678100 & -1.57466200 & 5.11188600\end{array}$

H $\quad 2.00087400 \quad-2.07456200 \quad 3.01733600$

H $\quad-0.07261200 \quad-3.43389100 \quad 3.07886200$

H $\quad \begin{array}{llll}1.12411600 & -4.03180200 & 4.24398000\end{array}$

H $\quad-0.26512000-3.07566700 \quad 4.80463600$

$\begin{array}{llll}\mathrm{H} & 2.65262700 & -0.67007400 & 4.99799400\end{array}$

H $\quad 1.34602400-1.40429300 \quad 5.93541100$

H $\quad 2.71231300 \quad-2.39902600 \quad 5.38763400$

$\begin{array}{llll}\text { C } & -1.90993900 & 0.84989100 & 2.73128700\end{array}$

$\begin{array}{llll}\text { C } & -1.73802500 & 1.50556200 & 4.10307000\end{array}$

$\begin{array}{llll}\text { C } & -2.69146800 & -0.45948100 & 2.79871300\end{array}$

$\begin{array}{llll}\mathrm{H} & -2.47230100 & 1.54236100 & 2.09127700\end{array}$

$\begin{array}{lllll}\mathrm{H} & -1.21698100 & 2.46514400 & 4.01777400\end{array}$

H $\quad-2.71781300 \quad 1.69510600 \quad 4.55377900$

$\begin{array}{llll}H & -1.16599500 & 0.85392200 & 4.76702200\end{array}$

$\begin{array}{llll}\mathrm{H} & -2.78019500 & -0.90812500 & 1.80493400\end{array}$

H $\quad-2.19729100-1.17301400 \quad 3.46085900$

$\begin{array}{llll}\mathrm{H} & -3.69808900 & -0.26800400 & 3.18325600\end{array}$

H $\quad-0.27582200 \quad 1.55848800 \quad 1.59241500$

C $\quad 0.18203800 \quad 3.75555500 \quad-0.11382900$

C $\quad 0.41042900 \quad 4.25678300 \quad-1.53477100$

H $\quad \begin{array}{lllll}\mathrm{C} & 1.36235400 & 3.88163600 & -1.92574500\end{array}$

H $\quad-0.38991500 \quad 3.90766200 \quad-2.19539200$

H $\quad 0.43313900 \quad 5.35226000 \quad-1.57054600$

$\begin{array}{lllll}\text { O } & 0.14138800 & 2.36074000 & -0.04325900\end{array}$

$\begin{array}{lllll}0 & -0.81660900 & -1.73476200 & -0.44884500\end{array}$

$\begin{array}{llll}\text { C } & -1.37791900 & -2.98879500 & -0.21751600\end{array}$

$\begin{array}{llll}\text { C } & -0.46585500 & -4.11471800 & -0.68981700\end{array}$

H $\quad-0.91821500-5.09529200-0.50100200$

H $\quad-0.27319200-4.02519500-1.76376500$

H $\quad 0.49645200 \quad-4.07266700 \quad-0.16899500$

$\begin{array}{llll}\text { C } & 2.59272200 & -0.41869800 & -3.03308400\end{array}$

C $\quad 2.14185100-0.13563200 \quad-4.45980300$

H $\quad 2.90843600-0.44128600 \quad-5.18115300$

H $\quad 1.21994500 \quad-0.68106400 \quad-4.68654000$

$\begin{array}{llll}\mathrm{H} & 1.94665400 & 0.93284700 & -4.59564000\end{array}$

C $\quad-2.63288300 \quad 1.07699800 \quad-3.19785900$

$\begin{array}{lllll}C & -3.94447400 & 0.36690700 & -2.88849300\end{array}$

H $\quad-4.32505600 \quad 0.67357100 \quad-1.90850300$

H $\quad-4.70557300 \quad 0.60217100 \quad-3.64135600$

H $\quad-3.79821500 \quad-0.71770600 \quad-2.87198700$

$\begin{array}{llll}H & 3.25149200 & -0.94124600 & 1.67259900\end{array}$

$\begin{array}{lllll}\mathrm{H} & 0.98464500 & 4.14491200 & 0.53717500\end{array}$

$\begin{array}{llll}\mathrm{H} & -0.75840800 & 4.18671600 & 0.27116300\end{array}$ $\begin{array}{lll}2.79465500 & 0.31758900 & 2.83827200\end{array}$

$\begin{array}{lll}-1.58775500 & -3.11862200 & 0.85753600\end{array}$

$\begin{array}{lll}-2.34896800 & -3.06742000 & -0.73407500\end{array}$

$\begin{array}{lll}-2.80546700 & 2.16471700 & -3.23638500\end{array}$

$\begin{array}{llll}3.53879500 & 0.11305800 & -2.83858500\end{array}$

$\begin{array}{llll}2.81017200 & -1.49371900 & -2.92436500\end{array}$

$\begin{array}{llll}-2.28263500 & 0.77950300 & -4.19883100\end{array}$

\section{dimer-A ( $\left.{ }^{\mathrm{BuOOH}}\right)$}

$H=-1461.241935 a u$

$\begin{array}{lllll}0 & -0.66909300 & 0.79420600 & 2.73989800\end{array}$

$\begin{array}{lllll}\mathrm{U} & & -0.90587200 & 0.51381800 & 0.32026700\end{array}$

$\begin{array}{lllll}0 & -2.61840100 & -0.64888100 & -0.46354300\end{array}$

$\begin{array}{lllll}0 & & 1.04319200 & 0.12378200 & -0.64840000\end{array}$

C $\quad-3.57233800-1.50640300 \quad-1.02830200$

C $\quad-3.31228800-1.63092000-2.53653600$

H $\quad-2.30703000-2.02766500-2.71464800$

$\mathrm{H} \quad-4.03520100-2.29592400 \quad-3.02269000$

H $\quad-3.37926600 \quad-0.64711100 \quad-3.01352900$

C $\quad-3.46129400 \quad-2.88745900-0.36593700$

$\mathrm{H} \quad-2.45879700 \quad-3.30079900 \quad-0.52170000$

$\mathrm{H} \quad-3.63384900 \quad-2.80229900 \quad 0.71237100$

H $\quad-4.19060400 \quad-3.59634400 \quad-0.77395600$

$\begin{array}{lllll}\text { C } & -4.97253700 & -0.92489500 & -0.78569500\end{array}$

H $\quad-5.15244700 \quad-0.81253400 \quad 0.28850200$

H $\quad-5.05535600 \quad 0.06380600 \quad-1.24913500$

$\mathrm{H} \quad-5.75840000-1.56529100-1.20217400$

$\begin{array}{llll}\text { C } & 2.20951400 & -0.15711800 & -1.37298800\end{array}$

$\begin{array}{llll}\text { C } & 1.88115400 & -0.17184400 & -2.87278500\end{array}$

$\mathrm{H} \quad 1.12509500-0.93504700 \quad-3.08562200$

H $\quad 1.48076300 \quad 0.80050600 \quad-3.17966000$

$\begin{array}{lllll}\text { H } & 2.76577400 & -0.38504600 & -3.48358600\end{array}$

C $\quad 2.74755500 \quad-1.52976700-0.94409900$

H $\quad 2.96201100 \quad-1.53026500 \quad 0.12998300$

H $\quad 2.00215800-2.30672400-1.14515900$

H $\quad 3.66838100 \quad-1.79241300-1.47708100$

C $\quad 3.25460500 \quad 0.92835900 \quad-1.07735800$

H $\quad 4.19103500 \quad 0.75312200 \quad-1.61915100$

H $\quad 2.86869300 \quad 1.91107500 \quad-1.36850900$

H $\quad 3.47730500 \quad 0.95515500 \quad-0.00553500$

$\begin{array}{lllll}0 & -1.44636600 & 2.86212400 & 0.70648700\end{array}$

$\begin{array}{llll}\text { U } & -1.20936000 & 3.14189000 & 3.12632400\end{array}$

$\begin{array}{lllll}0 & -3.15454200 & 3.53226700 & 4.10349000\end{array}$

$\begin{array}{lllll}0 & 0.50780800 & 4.29757500 & 3.90760500\end{array}$

$\begin{array}{llll}\text { C } & -4.31177300 & 3.81852500 & 4.84030900\end{array}$

$\begin{array}{llll}\text { C } & -3.96583000 & 3.83931200 & 6.33611600\end{array}$

H $\quad-3.20465700 \quad 4.60102500 \quad 6.53605000$

$\begin{array}{lllll}\mathrm{H} & -4.84236400 & 4.05860000 & 6.95636000\end{array}$

$\begin{array}{llll}H & -3.56518000 & 2.86706500 & 6.64308500\end{array}$

$\begin{array}{llll}\text { C } & -4.85251700 & 5.19019700 & 4.41163200\end{array}$

$\begin{array}{llll}\text { H } & -4.10426900 & 5.96713100 & 4.60183100\end{array}$

H $\quad-5.07796600 \quad 5.18663700 \quad 3.33982100$

H $\quad \begin{array}{llll}\text { H } & -5.76749400 & 5.45607200 & 4.95303200\end{array}$

$\begin{array}{llll}\text { C } & -5.36216600 & 2.73367500 & 4.56156000\end{array}$

H $\quad \begin{array}{llll}\text { H } & -5.59726200 & 2.70289400 & 3.49249200\end{array}$

H $\quad-4.97455600 \quad 1.75153800 \quad 4.85242400$

H $\quad-6.29190500 \quad 2.91295500 \quad 5.11345700$

C $\quad \begin{array}{llll}\text { C } & 1.48409600 & 5.13561900 & 4.46335300\end{array}$

C $\quad \begin{array}{llll}\text { C } & 1.68228300 & 4.76675300 & 5.94075400\end{array}$

$\begin{array}{lllll}\mathrm{H} & 0.74301500 & 4.89179200 & 6.49014600\end{array}$

$\begin{array}{llll}\mathrm{H} & 1.99337900 & 3.72028400 & 6.02899300\end{array}$

$\begin{array}{llll}\mathrm{H} & 2.44504200 & 5.39150900 & 6.41918000\end{array}$

$\begin{array}{llll}\text { C } & 1.02010700 & 6.59511200 & 4.35012100\end{array}$ 


$\begin{array}{lrrr}\mathrm{H} & 0.86458300 & 6.85999900 & 3.29863300 \\ \mathrm{H} & 0.07201400 & 6.73144100 & 4.88132100 \\ \mathrm{H} & 1.75328900 & 7.29158400 & 4.77245800 \\ \mathrm{C} & 2.80107800 & 4.94552800 & 3.69709500 \\ \mathrm{H} & 3.60079900 & 5.58066100 & 4.09487800 \\ \mathrm{H} & 3.12667100 & 3.90202100 & 3.76130400 \\ \mathrm{H} & 2.66080300 & 5.19213700 & 2.63937200 \\ \mathrm{C} & -1.93468600 & 3.83948800 & -0.21157400 \\ \mathrm{C} & -3.43265900 & 3.60984800 & -0.43875300 \\ \mathrm{C} & -1.70132400 & 5.23479800 & 0.38311200 \\ \mathrm{C} & -1.16564900 & 3.70622100 & -1.53104300 \\ \mathrm{H} & -3.61245700 & 2.60929000 & -0.84695000 \\ \mathrm{H} & -3.97799100 & 3.68903800 & 0.50752000 \\ \mathrm{H} & -3.85083900 & 4.34200900 & -1.13786700 \\ \mathrm{H} & -0.63850300 & 5.39056300 & 0.60112200 \\ \mathrm{H} & -2.02118100 & 6.02769400 & -0.30055200 \\ \mathrm{H} & -2.28487500 & 5.37081000 & 1.30528600 \\ \mathrm{H} & -1.48326700 & 4.45509600 & -2.26394700 \\ \mathrm{H} & -0.09023000 & 3.82247800 & -1.36319500 \\ \mathrm{H} & -1.34061300 & 2.72217400 & -1.98725400 \\ \mathrm{C} & -0.16956100 & -0.17877000 & 3.65687600 \\ \mathrm{C} & 1.32482000 & 0.07052500 & 3.88782100 \\ \mathrm{C} & -0.94343800 & -0.05915000 & 4.97476600 \\ \mathrm{C} & -0.38197500 & -1.57550900 & 3.05794900 \\ \mathrm{H} & 1.87273900 & 0.00037400 & 2.94232000 \\ \mathrm{H} & 1.49074200 & 1.07233000 & 4.29900700 \\ \mathrm{H} & 1.75117300 & -0.65768600 & 4.58612100 \\ \mathrm{H} & -2.01677400 & -0.18904400 & 4.80378400 \\ \mathrm{H} & -0.61765200 & -0.80586300 & 5.70629200 \\ \mathrm{H} & -0.78300500 & 0.92586300 & 5.43410900 \\ \mathrm{H} & -0.05447800 & -2.36572000 & 3.74110500 \\ \mathrm{H} & -1.44170300 & -1.74532800 & 2.83527900 \\ \mathrm{H} & 0.20744000 & -1.70111500 & 2.13810100\end{array}$

$\begin{array}{lrrr}\text { Cat }_{\mathrm{A}}\left({ }^{\mathrm{t}} \mathrm{BuOH}\right) & & \\ \mathrm{H}=--730.576978 \mathrm{au} & & \\ \mathrm{U} & 0.92637100 & 2.25931000 & -0.41360100 \\ \mathrm{C} & 3.50119900 & 0.68018300 & -3.64627300 \\ \mathrm{H} & 3.81967700 & 1.71505100 & -3.81157200 \\ \mathrm{H} & 4.20813500 & 0.01803400 & -4.15874000 \\ \mathrm{H} & 2.51426800 & 0.54964300 & -4.10224500 \\ \mathrm{C} & 2.95994700 & -1.05869000 & -1.91371600 \\ \mathrm{H} & 1.96828700 & -1.20611700 & -2.35428600 \\ \mathrm{H} & 3.64632800 & -1.78553400 & -2.36250600 \\ \mathrm{H} & 2.89290800 & -1.26915100 & -0.84099100 \\ \mathrm{C} & 4.80823700 & 0.59462400 & -1.49866800 \\ \mathrm{H} & 5.13777700 & 1.62882900 & -1.64450500 \\ \mathrm{H} & 4.75354400 & 0.40320100 & -0.42198900 \\ \mathrm{H} & 5.56504100 & -0.07072000 & -1.92932200 \\ \mathrm{C} & -2.09957900 & 0.09419200 & -2.64192300 \\ \mathrm{H} & -1.19666700 & -0.50713000 & -2.79016100 \\ \mathrm{H} & -2.97003700 & -0.52730200 & -2.88065700 \\ \mathrm{H} & -2.07075000 & 0.93077100 & -3.34810300 \\ \mathrm{C} & -3.40619100 & 1.48441500 & -1.00322500 \\ \mathrm{H} & -4.32503200 & 0.91359800 & -1.17838500 \\ \mathrm{H} & -3.43468400 & 1.87711600 & 0.01865000 \\ \mathrm{H} & -3.39245200 & 2.33302700 & -1.69548900 \\ \mathrm{C} & -2.16795200 & -0.55439800 & -0.21362500 \\ \mathrm{H} & -2.18275000 & -0.18150400 & 0.81579300 \\ \mathrm{H} & -3.04474400 & -1.19517700 & -0.35991500 \\ \mathrm{H} & -1.27062800 & -1.16900200 & -0.34260400 \\ \mathrm{C} & 1.66513200 & 0.00077600 & 3.01101200 \\ \mathrm{H} & 0.77205000 & -0.47647000 & 2.59397300\end{array}$

$\begin{array}{lll}2.52158900 & -0.26553800 & 2.38264800\end{array}$ $\begin{array}{lll}1.83523600 & -0.40825700 & 4.01334300\end{array}$ $\begin{array}{lll}0.26956700 & 1.89210700 & 3.90306000\end{array}$ $\begin{array}{lll}0.12797900 & 2.97812600 & 3.91066600\end{array}$ $\begin{array}{lll}-0.63550500 & 1.43320800 & 3.49186100\end{array}$ $\begin{array}{lll}0.38717100 & 1.55429600 & 4.93888300\end{array}$ $\begin{array}{lll}2.74930200 & 2.18963900 & 3.60426800\end{array}$ $\begin{array}{llll}2.95898900 & 1.86307100 & 4.62906500\end{array}$ $\begin{array}{lll}3.61436400 & 1.94258900 & 2.98000700\end{array}$ $\begin{array}{llll}2.63132800 & 3.27855200 & 3.60904400\end{array}$ $\begin{array}{llll}2.51413100 & 1.26926400 & -1.55324000\end{array}$ $\begin{array}{lll}1.27444600 & 1.99292500 & 1.73531800\end{array}$ $\begin{array}{lll}-1.02090400 & 1.41521400 & -0.96178700\end{array}$ $\begin{array}{lll}-2.15244100 & 0.62049900 & -1.20108500\end{array}$ $\begin{array}{lll}3.43047800 & 0.38425300 & -2.14156500\end{array}$ $\begin{array}{lll}1.48544400 & 1.52461400 & 3.04110900\end{array}$

\section{Int-1A ('BuOH)}

$H=-1114.924459$ au

$\begin{array}{llll}\mathrm{N} & -0.94484500 & 3.14654200 & 1.02399600\end{array}$

$\begin{array}{llll}\text { C } & -1.04911100 & 3.51666500 & 2.21054200\end{array}$

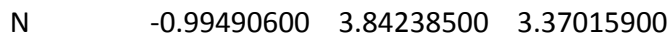

$\begin{array}{lllll}\text { O } & 0.38481500 & -0.28453800 & 2.46081400\end{array}$

C $\quad 0.93300700 \quad-0.98415400 \quad 3.53932600$

C $\quad-0.09219800 \quad-1.02655800 \quad 4.68327500$

H $\quad 0.28856100 \quad-1.56791900 \quad 5.55723200$

H $\quad-0.34623700 \quad-0.00756200 \quad 4.99557100$

H $\quad-1.01031200 \quad-1.51862100 \quad 4.34536100$

$\begin{array}{llll}\text { C } & 1.27605800 & -2.41597700 & 3.10049900\end{array}$

H $\quad 1.99780300 \quad-2.39317000 \quad 2.27686300$

H $\quad 1.70912200-3.00330000 \quad 3.91857100$

$\begin{array}{llll}\mathrm{H} & 0.37369400 & -2.92860600 & 2.75051600\end{array}$

$\begin{array}{llll}\text { C } & 2.20770800 & -0.26625200 & 4.00920200\end{array}$

$\begin{array}{llll}\mathrm{H} & 1.97137500 & 0.76080700 & 4.30787000\end{array}$

$\begin{array}{llll}\mathrm{H} & 2.67659800 & -0.77308100 & 4.86083700\end{array}$

H $\quad 2.93532900 \quad-0.22298900 \quad 3.19186300$

$\begin{array}{lllll}\text { U } & -0.29616300 & 0.54437100 & 0.51404000\end{array}$

$\begin{array}{lllll}0 & -2.18965800 & -0.39204100 & -0.18528400\end{array}$

$\begin{array}{lllll}0 & 1.26744300 & 0.55966800 & -1.06856400\end{array}$

C $\quad-3.21542100-1.25017600-0.59071900$

C $\quad-3.51212400-1.01698900-2.08013700$

H $\quad-2.61081500-1.20073300-2.67422300$

H $\quad-4.30881000-1.67383900-2.44858000$

H $\quad \begin{array}{llll}H & -3.82072100 & 0.02108500 & -2.24492400\end{array}$

C $\quad-2.78066300 \quad-2.70773600-0.37223900$

H $\quad-1.87873200-2.92235800-0.95591800$

H $\quad-2.55140700 \quad-2.87669000 \quad 0.68540500$

H $\quad-3.55903400 \quad-3.41894200 \quad-0.67241800$

$\begin{array}{llll}\text { C } & -4.47244300 & -0.95558500 & 0.24270800\end{array}$

H $\quad-4.26111000-1.11213000 \quad 1.30590600$

$\begin{array}{lllll}H & -4.77584100 & 0.08805300 & 0.10576100\end{array}$

H $\quad-5.31410900-1.59852100 \quad-0.04003200$

$\begin{array}{llll}\text { C } & 2.27342200 & 0.24412900 & -1.98690000\end{array}$

$\begin{array}{lllll}C & 1.87054300 & 0.76606200 & -3.37468700\end{array}$

$\begin{array}{lllll}\mathrm{H} & 0.92132300 & 0.31507100 & -3.68236800\end{array}$

$\begin{array}{lllll}\mathrm{H} & 1.73775900 & 1.85300100 & -3.34341700\end{array}$

H $\quad 2.62557800 \quad 0.53553600 \quad-4.13525300$

C $\quad 2.45481000 \quad-1.28055300 \quad-2.04164300$

H $\quad 2.72585600-1.66241600 \quad-1.05124100$

H $\quad \begin{array}{llll}1.51829800 & -1.75897000 & -2.34842600\end{array}$

H $\quad 3.23842600-1.57686000 \quad-2.74861400$

$\begin{array}{lllll}\text { C } & 3.58620600 & 0.90735700 & -1.54242900\end{array}$ 


$\begin{array}{lrrr}\text { H } & 4.40936300 & 0.69527100 & -2.23470000 \\ \text { H } & 3.45664200 & 1.99352200 & -1.48529700 \\ \text { H } & 3.87081000 & 0.54717900 & -0.54811800 \\ \text { C } & -1.91113600 & 3.76418800 & 4.50554700 \\ \text { C } & -3.15429700 & 2.93575200 & 4.19890700 \\ \text { C } & -2.24050600 & 5.18394000 & 4.96391100 \\ \text { H } & -1.33701000 & 3.27096600 & 5.29922700 \\ \text { H } & -2.88772400 & 1.92118100 & 3.88881600 \\ \text { H } & -3.78431100 & 2.86136100 & 5.09023800 \\ \text { H } & -3.74679500 & 3.40138700 & 3.40380000 \\ \text { H } & -1.32788100 & 5.75197900 & 5.16113900 \\ \text { H } & -2.81964900 & 5.71284800 & 4.20008200 \\ \text { H } & -2.83393600 & 5.14988900 & 5.88200100 \\ \text { C } & -1.30420300 & 4.05515800 & -0.10528500 \\ \text { C } & -1.48764100 & 3.21320300 & -1.36131200 \\ \text { C } & -0.22771600 & 5.12283600 & -0.27662000 \\ \text { H } & -2.25883800 & 4.53824100 & 0.14091700 \\ \text { H } & -2.24285800 & 2.43298800 & -1.21612300 \\ \text { H } & -1.81648200 & 3.84337900 & -2.19262000 \\ \text { H } & -0.54246700 & 2.74381400 & -1.66386900 \\ \text { H } & -0.11338500 & 5.72394200 & 0.63060100 \\ \text { H } & 0.73701200 & 4.66126300 & -0.50894200 \\ \text { H } & -0.49331900 & 5.79443100 & -1.09908600\end{array}$

$\begin{array}{lrrr}\text { TS }_{1 \mathrm{~A}-2 \mathrm{~A}}\left({ }^{\mathrm{t} B u O H}\right) & & \\ \mathrm{H}=-1114.899304 \mathrm{au} & & \\ \mathrm{N} & -0.82893000 & 2.55885600 & 0.98668800 \\ \mathrm{C} & -0.81481500 & 2.58431800 & 2.28130400 \\ \mathrm{~N} & -0.94116700 & 3.31158600 & 3.26286200 \\ \mathrm{O} & -0.42325700 & 0.72684700 & 2.57936000 \\ \mathrm{C} & 0.55774800 & 0.19678800 & 3.46885500 \\ \mathrm{C} & -0.12886700 & -0.32629800 & 4.73528600 \\ \mathrm{H} & 0.59435800 & -0.78018100 & 5.42173900 \\ \mathrm{H} & -0.64020200 & 0.47916000 & 5.26811900 \\ \mathrm{H} & -0.87616900 & -1.08207500 & 4.47327200 \\ \mathrm{C} & 1.23390100 & -0.98998200 & 2.75483300 \\ \mathrm{H} & 1.78466200 & -0.66553400 & 1.86044900 \\ \mathrm{H} & 1.96075300 & -1.49306100 & 3.40090800 \\ \mathrm{H} & 0.48733500 & -1.74986700 & 2.47784400 \\ \mathrm{C} & 1.62804100 & 1.23987800 & 3.80817100 \\ \mathrm{H} & 1.19443900 & 2.10399900 & 4.31591100 \\ \mathrm{H} & 2.39667800 & 0.80936600 & 4.45890100 \\ \mathrm{H} & 2.11613700 & 1.59806100 & 2.89571900 \\ \mathrm{U} & -0.48622900 & 0.18633800 & 0.21887400 \\ \mathrm{O} & -2.31699000 & -0.76338100 & -0.59832800 \\ \mathrm{O} & 1.41750100 & -0.02684500 & -0.89904100 \\ \mathrm{C} & -3.43974000 & -1.42051800 & -1.11550400 \\ \mathrm{C} & -3.66116400 & -0.97626100 & -2.56885700 \\ \mathrm{H} & -2.77994200 & -1.21426000 & -3.17396300 \\ \mathrm{H} & -4.53073900 & -1.46880100 & -3.01885900 \\ \mathrm{H} & -3.82138700 & 0.10638800 & -2.61161400 \\ \mathrm{C} & -3.19970400 & -2.93695900 & -1.06756200 \\ \mathrm{H} & -2.31383600 & -3.19667100 & -1.65706800 \\ \mathrm{H} & -3.02954200 & -3.25838200 & -0.03406600 \\ \mathrm{H} & -4.05260600 & -3.49959200 & -1.46402900 \\ \mathrm{C} & -4.66883200 & -1.06122200 & -0.26730000 \\ \mathrm{H} & -4.50809200 & -1.36135800 & 0.77366100 \\ \mathrm{H} & -4.83530900 & 0.02105800 & -0.28642600 \\ \mathrm{H} & -5.57711100 & -1.55498300 & -0.63149500 \\ \mathrm{C} & 2.47353100 & -0.25427600 & -1.79080200 \\ \mathrm{C} & 1.98838500 & 0.00305000 & -3.22527300 \\ \mathrm{H} & 1.15124600 & -0.66177600 & -3.46446600\end{array}$

$\begin{array}{lll}1.64271200 & 1.03769400 & -3.32583200\end{array}$ $\begin{array}{lll}2.78140600 & -0.16483700 & -3.96287000\end{array}$ $2.94233800-1.71063700-1.65571500$ $\begin{array}{llll}3.27292100 & -1.90682000 & -0.63011200\end{array}$ $\begin{array}{llll}2.11828300 & -2.39473100 & -1.88621700\end{array}$ $\begin{array}{llll}3.77417300 & -1.93696100 & -2.33236800\end{array}$ $\begin{array}{llll}3.62822900 & 0.69942400 & -1.45188300\end{array}$ $\begin{array}{llll}4.48297100 & 0.56192100 & -2.12403200\end{array}$ $\begin{array}{llll}3.29211100 & 1.73859400 & -1.52996000\end{array}$ $\begin{array}{llll}3.96734600 & 0.52958900 & -0.42461700\end{array}$ $\begin{array}{lll}-1.48152600 & 3.16642300 & 4.59258500\end{array}$ $\begin{array}{lll}-2.88274100 & 2.55311100 & 4.56448200\end{array}$ $\begin{array}{lll}-1.46844200 & 4.53066000 & 5.28094600\end{array}$ $\begin{array}{lll}-0.82066900 & 2.49660400 & 5.16224300\end{array}$ $\begin{array}{lll}-2.86098200 & 1.56786300 & 4.09078600\end{array}$ $\begin{array}{lll}-3.27564300 & 2.44249300 & 5.58073900\end{array}$ $\begin{array}{lll}-3.56748900 & 3.19539700 & 4.00059600\end{array}$ $\begin{array}{llll}-0.45824300 & 4.94936900 & 5.29389900\end{array}$ $\begin{array}{llll}-2.12009100 & 5.23291900 & 4.75099100\end{array}$ $\begin{array}{lll}-1.82181400 & 4.44266300 & 6.31299800\end{array}$ $\begin{array}{lll}-1.34154800 & 3.77413500 & 0.32389800\end{array}$ $\begin{array}{llll}-1.79047900 & 3.39934700 & -1.08496900\end{array}$ $\begin{array}{llll}-0.27566000 & 4.86899200 & 0.30874500\end{array}$ $\begin{array}{lll}-2.21110900 & 4.14287500 & 0.88751800\end{array}$ $\begin{array}{llll}-2.57278100 & 2.63212700 & -1.06406300\end{array}$ $\begin{array}{llll}-2.19171400 & 4.26959200 & -1.61326300\end{array}$ $\begin{array}{llll}-0.94284700 & 3.02614700 & -1.67579200\end{array}$ $\begin{array}{llll}0.02868000 & 5.12053200 & 1.32823500\end{array}$ $\begin{array}{llll}0.60833800 & 4.53419400 & -0.24476800\end{array}$ $\begin{array}{llll}-0.65896800 & 5.77699800 & -0.16948700\end{array}$

$\begin{array}{lrrr}\text { Int-2A ('BuOH) } & & \\ H=-1114.907948 \text { au } & & \\ \text { N } & -0.48937600 & 2.23444900 & 1.12135500 \\ \mathrm{C} & -0.87901400 & 2.12397700 & 2.40842000 \\ \mathrm{~N} & -1.60986300 & 2.91750900 & 3.08533900 \\ \mathrm{O} & -0.41699600 & 0.83239400 & 2.91963700 \\ \mathrm{C} & 0.82966600 & 0.75707900 & 3.69571300 \\ \mathrm{C} & 0.52360700 & -0.14383100 & 4.88953000 \\ \mathrm{H} & 1.42747300 & -0.31453400 & 5.48278200 \\ \mathrm{H} & -0.23168300 & 0.30939000 & 5.53590000 \\ \mathrm{H} & 0.14579600 & -1.11554700 & 4.55523600 \\ \mathrm{C} & 1.89405500 & 0.10439100 & 2.80748600 \\ \mathrm{H} & 2.10843700 & 0.71546400 & 1.92266100 \\ \mathrm{H} & 2.83733100 & -0.01312000 & 3.34898600 \\ \mathrm{H} & 1.59969300 & -0.91314200 & 2.50533200 \\ \mathrm{C} & 1.31401200 & 2.13298700 & 4.13976100 \\ \mathrm{H} & 0.58707800 & 2.62363100 & 4.78834500 \\ \mathrm{H} & 2.24836800 & 2.01771400 & 4.69784700 \\ \mathrm{H} & 1.50436000 & 2.77980800 & 3.27929400 \\ \mathrm{U} & -0.29519300 & -0.10090000 & 0.41169600 \\ \mathrm{O} & -2.22411600 & -0.80949500 & -0.35439400 \\ \mathrm{O} & 1.34633300 & -0.36730500 & -1.03164200 \\ \mathrm{C} & -3.40515200 & -1.22980300 & -0.98597100 \\ \mathrm{C} & -3.20882800 & -1.18863800 & -2.50748800 \\ \mathrm{H} & -2.37750500 & -1.83942700 & -2.79857100 \\ \mathrm{H} & -4.10695500 & -1.51811400 & -3.04200000 \\ \mathrm{H} & -2.97317900 & -0.16934000 & -2.83120000 \\ \mathrm{C} & -3.71688200 & -2.66500300 & -0.53836700 \\ \mathrm{H} & -2.89359100 & -3.33320900 & -0.81344600 \\ \mathrm{H} & -3.84008700 & -2.70137500 & 0.54929400 \\ \mathrm{H} & -4.63492700 & -3.04519900 & -1.00026100 \\ \mathrm{C} & -4.55049900 & -0.29400300 & -0.57668900\end{array}$




$\begin{array}{lrrr}\text { H } & -4.67763200 & -0.30764900 & 0.51064600 \\ \mathrm{H} & -4.32506700 & 0.73397700 & -0.87827300 \\ \mathrm{H} & -5.49969000 & -0.58771000 & -1.03897000 \\ \mathrm{C} & 2.26646600 & -0.54902100 & -2.07488200 \\ \mathrm{C} & 1.60517100 & -0.16342300 & -3.40534500 \\ \mathrm{H} & 0.72265900 & -0.78658900 & -3.58335300 \\ \mathrm{H} & 1.28377700 & 0.88297900 & -3.37737400 \\ \mathrm{H} & 2.29026800 & -0.28864700 & -4.25143200 \\ \mathrm{C} & 2.69028800 & -2.02440600 & -2.11163200 \\ \mathrm{H} & 3.14687200 & -2.30978500 & -1.15756300 \\ \mathrm{H} & 1.81642200 & -2.66337500 & -2.27830800 \\ \mathrm{H} & 3.41523400 & -2.22075500 & -2.90959600 \\ \mathrm{C} & 3.49201900 & 0.34055200 & -1.82302500 \\ \mathrm{H} & 4.24016300 & 0.23718900 & -2.61710400 \\ \mathrm{H} & 3.18979100 & 1.39156400 & -1.77019300 \\ \mathrm{H} & 3.96394700 & 0.07330500 & -0.87145000 \\ \mathrm{C} & -2.13294300 & 2.62682000 & 4.40186900 \\ \mathrm{C} & -3.58667600 & 2.15548700 & 4.27547500 \\ \mathrm{C} & -2.04903400 & 3.88747900 & 5.26555000 \\ \mathrm{H} & -1.56999800 & 1.82254900 & 4.90182400 \\ \mathrm{H} & -3.64567600 & 1.22917300 & 3.69561500 \\ \mathrm{H} & -4.02986300 & 1.97363300 & 5.26125800 \\ \mathrm{H} & -4.18356400 & 2.91489800 & 3.76002800 \\ \mathrm{H} & -1.01426600 & 4.22962700 & 5.37069200 \\ \mathrm{H} & -2.61709100 & 4.69835900 & 4.79803000 \\ \mathrm{H} & -2.45614900 & 3.70867000 & 6.26687400 \\ \mathrm{C} & -0.92384200 & 3.43584600 & 0.41134900 \\ \mathrm{C} & -0.87916100 & 3.16490900 & -1.09094400 \\ \mathrm{C} & -0.05766000 & 4.64135100 & 0.78497600 \\ \mathrm{H} & -1.95916800 & 3.66536000 & 0.70504500 \\ \mathrm{H} & -1.55551500 & 2.34600700 & -1.37062500 \\ \mathrm{H} & -1.18941800 & 4.04559200 & -1.66245300 \\ \mathrm{H} & 0.13801800 & 2.90461900 & -1.41034400 \\ \mathrm{H} & -0.11509400 & 4.82058500 & 1.86163000 \\ \mathrm{H} & 0.98810000 & 4.46352100 & 0.50858200 \\ \mathrm{H} & -0.39980500 & 5.54619000 & 0.26985400\end{array}$

\begin{tabular}{|c|c|c|c|}
\hline \multicolumn{4}{|c|}{ Int-3A ( $\left.{ }^{t} \mathrm{BuOH}\right)$} \\
\hline \multicolumn{4}{|c|}{$H=-1348.386727 a u$} \\
\hline$N$ & -0.17529800 & 1.13391100 & 2.00376800 \\
\hline C & -0.08308200 & -0.01061800 & 2.70730800 \\
\hline $\mathrm{N}$ & -0.71285600 & -0.39365800 & 3.75237900 \\
\hline $\mathrm{O}$ & 0.82314400 & -0.91206700 & 1.98929700 \\
\hline C & 2.26538900 & -0.87033700 & 2.31433500 \\
\hline C & 2.80159500 & -2.18468200 & 1.75784300 \\
\hline $\mathrm{H}$ & 3.87698100 & -2.26493700 & 1.94504500 \\
\hline $\mathrm{H}$ & 2.30145100 & -3.03403400 & 2.23186500 \\
\hline $\mathrm{H}$ & 2.64438000 & -2.24158500 & 0.67577600 \\
\hline C & 2.92221300 & 0.32884400 & 1.62531900 \\
\hline $\mathrm{H}$ & 2.44801900 & 1.25951500 & 1.94719000 \\
\hline $\mathrm{H}$ & 3.98494700 & 0.36976100 & 1.88511200 \\
\hline $\mathrm{H}$ & 2.87184700 & 0.24402200 & 0.53190700 \\
\hline C & 2.48893300 & -0.78479300 & 3.82096000 \\
\hline $\mathrm{H}$ & 2.08771000 & -1.65835800 & 4.33809400 \\
\hline $\mathrm{H}$ & 3.56504200 & -0.74103900 & 4.01600900 \\
\hline $\mathrm{H}$ & 2.02969500 & 0.11231700 & 4.24336200 \\
\hline U & 0.10853400 & 0.06006500 & -0.32984200 \\
\hline 0 & -1.76415400 & -0.83189600 & -1.07787000 \\
\hline $\mathrm{O}$ & 1.85244000 & -0.37850600 & -1.66604300 \\
\hline C & -2.84903600 & -1.54425000 & -1.60686100 \\
\hline C & -4.04372100 & -0.59209700 & -1.76083200 \\
\hline $\mathrm{H}$ & -3.78596000 & 0.23012800 & -2.43740300 \\
\hline$H$ & -4.92383800 & -1.10469100 & -2.16555000 \\
\hline
\end{tabular}

$-4.31053600-0.16425200-0.78908600$ $-2.45996300-2.12007000-2.97620000$ $\begin{array}{llll}-2.18233900 & -1.31215100 & -3.66177600\end{array}$ $\begin{array}{lll}-1.59829000 & -2.78804000 & -2.87270700\end{array}$ $\begin{array}{lll}-3.28100100 & -2.68742900 & -3.42930800\end{array}$ $\begin{array}{lll}-3.21513600 & -2.68481200 & -0.64652400\end{array}$ $\begin{array}{llll}-2.36185100 & -3.36026900 & -0.52223300\end{array}$ $\begin{array}{lll}-3.47562100 & -2.27900600 & 0.33622000\end{array}$ $\begin{array}{lll}-4.06500000 & -3.27116100 & -1.01396700\end{array}$ $\begin{array}{llll}2.78886900 & -0.80323800 & -2.61747800\end{array}$ $\begin{array}{llll}2.59718700 & -2.30375100 & -2.88608500\end{array}$ $\begin{array}{llll}2.73596900 & -2.87414400 & -1.96129000\end{array}$ $\begin{array}{llll}1.58351500 & -2.49411400 & -3.25407400\end{array}$ $3.30936000-2.68177900-3.62862300$ $\begin{array}{llll}4.21029400 & -0.54792300 & -2.09334200\end{array}$ $\begin{array}{llll}4.34627800 & 0.51716000 & -1.87691200\end{array}$ $4.38147100-1.10783200-1.16806400$ $\begin{array}{llll}4.97314200 & -0.85080300 & -2.81977000\end{array}$ $\begin{array}{llll}2.57828800 & -0.01928700 & -3.92191000\end{array}$ $\begin{array}{llll}3.28500800 & -0.32459900 & -4.70208700\end{array}$ $\begin{array}{llll}1.56256700 & -0.18129800 & -4.29851300\end{array}$ $\begin{array}{llll}2.71431400 & 1.05309500 & -3.74271200\end{array}$ $\begin{array}{lll}-0.67773500 & -1.74363800 & 4.27496300\end{array}$ $\begin{array}{lll}-1.98550600 & -2.45735400 & 3.90979600\end{array}$ $-0.50365400-1.69612900 \quad 5.79493200$ $\begin{array}{llll}0.14523100 & -2.33270300 & 3.84158200\end{array}$ $\begin{array}{lll}-2.10213400 & -2.52306800 & 2.82369600\end{array}$ $\begin{array}{lll}-2.00741100 & -3.47409800 & 4.31916100\end{array}$ $\begin{array}{lll}-2.84296700 & -1.90512000 & 4.30891000\end{array}$ $\begin{array}{lll}0.43396600 & -1.20471000 & 6.07238500\end{array}$ $\begin{array}{llll}-1.32249900 & -1.12566800 & 6.24617000\end{array}$ $\begin{array}{lll}-0.50525200 & -2.70359600 & 6.22604900\end{array}$ $\begin{array}{lll}-1.05891700 & 2.18371400 & 2.51711200\end{array}$ $\begin{array}{lll}-0.61216700 & 2.72371000 & 3.88042600\end{array}$ $\begin{array}{lll}-2.54433500 & 1.79998800 & 2.52067300\end{array}$ $\begin{array}{lll}-0.95740600 & 3.02897400 & 1.80945100\end{array}$ $\begin{array}{llll}0.43421900 & 3.04487600 & 3.84117000\end{array}$ $\begin{array}{llll}-1.22433300 & 3.58299900 & 4.17699700\end{array}$ $\begin{array}{lll}-0.70994700 & 1.94379900 & 4.63885000\end{array}$ $\begin{array}{lll}-2.86290700 & 1.46613600 & 1.52657100\end{array}$ $\begin{array}{lll}-2.71299500 & 0.98544700 & 3.22778500\end{array}$ $\begin{array}{lll}-3.16628200 & 2.65559900 & 2.80796600\end{array}$ $\begin{array}{llll}-0.15671800 & 3.04416600 & 0.09854000\end{array}$ $\begin{array}{llll}-0.35639800 & 3.52894900 & -1.84278600\end{array}$ $\begin{array}{llll}0.85097100 & 4.46061000 & -1.91418000\end{array}$ $\begin{array}{llll}0.73830400 & 5.17472300 & -2.73563400\end{array}$ $\begin{array}{llll}0.95922200 & 5.03426200 & -0.98693500\end{array}$ $\begin{array}{llll}1.76872200 & 3.88845700 & -2.07586800\end{array}$ $\begin{array}{llll}-1.64642200 & 4.29514600 & -1.56236100\end{array}$ $\begin{array}{llll}-1.86267600 & 4.99047800 & -2.37894500\end{array}$ $\begin{array}{llll}-2.49026600 & 3.60702400 & -1.46142400\end{array}$ $\begin{array}{llll}-1.56529500 & 4.88277700 & -0.64068700\end{array}$ $\begin{array}{llll}-0.47640700 & 2.66981100 & -3.09422200\end{array}$ $\begin{array}{llll}0.42484200 & 2.06544600 & -3.23627700\end{array}$ $\begin{array}{llll}-1.34201300 & 2.00297400 & -3.02760800\end{array}$ $\begin{array}{llll}-0.60333400 & 3.30240700 & -3.97703400\end{array}$ $\begin{array}{llll}-0.13653900 & 2.58370000 & -0.75393700\end{array}$

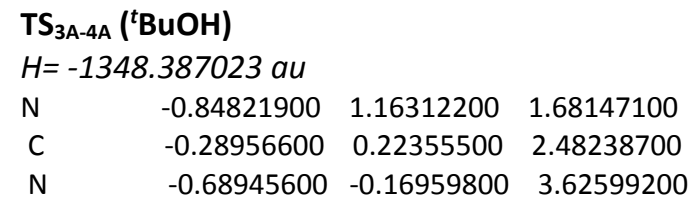




\section{Int-4A ('BuOH)}

$H=-1348.399433 a u$

$\begin{array}{llll}\mathrm{N} & -0.72532300 & 1.15827000 & 2.01009800\end{array}$

$\begin{array}{lllll}\text { C } & & -0.10211800 & 0.13758700 & 2.69515000\end{array}$

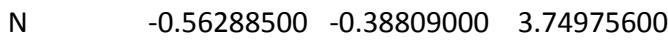

$\begin{array}{lllll}\text { O } & 0.98194400 & -0.34074700 & 1.92836100\end{array}$

$\begin{array}{lllll}\text { C } & 2.39954500 & 0.00526000 & 2.26269500\end{array}$

C $\quad 3.14377500 \quad-1.31780800 \quad 2.39843900$

H $\quad 4.21563900-1.12596500 \quad 2.50783000$

H $\quad 2.81182800-1.88744700 \quad 3.26975600$

H $\quad 3.00064600 \quad-1.92898500 \quad 1.50282800$

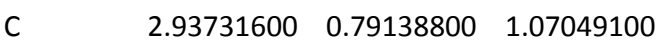

$\begin{array}{llll}\mathrm{H} & 2.34920900 & 1.69515300 & 0.88379900\end{array}$

$\begin{array}{llll}\mathrm{H} & 3.97290400 & 1.09111700 & 1.25942700\end{array}$

$\begin{array}{llll}\mathrm{H} & 2.93828400 & 0.17792000 & 0.16105300\end{array}$

$\begin{array}{lllll}\text { C } & 2.46607100 & 0.83364100 & 3.53765500\end{array}$

$\begin{array}{llll}\mathrm{H} & 2.07574100 & 0.29194400 & 4.40112000\end{array}$

$\begin{array}{llll}\mathrm{H} & 3.51249900 & 1.08236100 & 3.73678700\end{array}$

H $\quad \begin{array}{llll}\text { H } & 1.91451300 & 1.77309400 & 3.43558800\end{array}$

U $\quad 0.01967400-0.09791300-0.62138700$

O $\quad-1.92667700 \quad-0.82269000 \quad-1.38388500$

$\begin{array}{lllll}0 & 1.67933200 & -0.95736800 & -1.82294600\end{array}$

C $\quad-3.07769100-1.31354100-2.01113300$

C $\quad-4.16805200-0.23226100 \quad-1.98728700$

H $\quad \begin{array}{lllll}\text { H } & -3.81867200 & 0.66498100 & -2.50891300\end{array}$

H $\quad-5.09187400 \quad-0.57264700 \quad-2.46919100$

H $\quad-4.40225100 \quad 0.04508000 \quad-0.95414200$

C $\quad-2.74637700 \quad-1.68386600 \quad-3.46438400$

H $\quad-2.38507100-0.80190200-4.00403900$

$\begin{array}{llll}\mathrm{H} & -1.95856400 & -2.44413000 & -3.48817000\end{array}$

H $\quad-3.62057700 \quad-2.07704000 \quad-3.99615600$

C $\quad-3.56337100-2.55973300-1.25546800$

H $\quad-2.78504600 \quad-3.33047600 \quad-1.26345700$

$\mathrm{H} \quad-3.78148200-2.30556100 \quad-0.21265800$

H $\quad-4.47008100-2.98385500-1.70210300$

C $\quad 2.47398400 \quad-1.56853200 \quad-2.80107300$

C $\quad 2.28355500 \quad-3.09115600 \quad-2.72737400$

H $\quad 2.56389900-3.45792100 \quad-1.73376800$

$\mathrm{H} \quad \begin{array}{llll}\mathrm{H} & 1.23311200 & -3.34784400 & -2.90112500\end{array}$

H $\quad 2.89293500-3.61771900 \quad-3.47095000$

C $\quad 3.94721600 \quad-1.21657800 \quad-2.54549200$

H $\quad 4.08277600 \quad-0.13027300 \quad-2.57166800$

$\mathrm{H} \quad 4.25641100-1.57587700 \quad-1.55782100$

H $\quad 4.61049200-1.66487500-3.29408300$

$\begin{array}{llll}\text { C } & 2.05563800 & -1.06056000 & -4.18896400\end{array}$

H $\quad 2.65662200 \quad-1.51068000 \quad-4.98753100$

H $\quad 1.00351700 \quad-1.29944300 \quad-4.37663500$ 


\section{dimer-B ( ${ }^{\text {t } \mathrm{BuOH})}$}

$H=-1938.445232 a u$

$\begin{array}{lrrr}\text { O } & -0.71582700 & 0.87222100 & 2.72844300 \\ \text { O } & -1.42978000 & 2.73266900 & 0.81798000 \\ \text { U } & -1.15691800 & 3.12210100 & 3.27935500 \\ \text { C } & -1.90168400 & 3.73442300 & -0.12065900 \\ \text { C } & -3.20348600 & 3.22976600 & -0.74748200 \\ \text { C } & -2.16686300 & 5.04728900 & 0.61795700 \\ \text { C } & -0.82236600 & 3.95798700 & -1.18207800 \\ \text { H } & -3.03462100 & 2.30559200 & -1.30886900 \\ \text { H } & -3.94857000 & 3.04211700 & 0.03161200 \\ \text { H } & -3.61618700 & 3.96738000 & -1.44433600 \\ \text { H } & -1.25347400 & 5.41211700 & 1.09814100 \\ \text { H } & -2.51015400 & 5.81513600 & -0.08307700 \\ \text { H } & -2.94187100 & 4.91521600 & 1.37922300 \\ \text { H } & -1.13306200 & 4.72696500 & -1.89773700 \\ \text { H } & 0.10752100 & 4.28734200 & -0.70907600 \\ \text { H } & -0.63156200 & 3.03690200 & -1.74048100 \\ \text { C } & -0.38488300 & -0.18062200 & 3.67108900 \\ \text { C } & 0.92964200 & 0.18333300 & 4.36291100 \\ \text { C } & -1.53094700 & -0.30447700 & 4.67761800 \\ \text { C } & -0.21682800 & -1.50701200 & 2.92676600 \\ \text { H } & 1.73036500 & 0.27371300 & 3.62320000 \\ \text { H } & 0.83260600 & 1.12948300 & 4.90262600 \\ \text { H } & 1.21832300 & -0.58781500 & 5.08550400 \\ \text { H } & -2.46459100 & -0.54090700 & 4.15808600\end{array}$

$\begin{array}{lll}-1.33206000 & -1.10255600 & 5.40127800\end{array}$ $\begin{array}{lll}-1.65904000 & 0.62661000 & 5.23784600\end{array}$ $\begin{array}{llll}0.04607900 & -2.30494700 & 3.62901600\end{array}$ $\begin{array}{lll}-1.14624900 & -1.79369700 & 2.42523400\end{array}$ $\begin{array}{lll}0.58708000 & -1.43723400 & 2.18655500\end{array}$ $\begin{array}{lll}-0.93452300 & 0.50020000 & 0.26938000\end{array}$ $\begin{array}{lll}-1.18932200 & 0.61566100 & -1.87646900\end{array}$ $\begin{array}{lll}-2.52395600 & -0.92275800 & 0.57304000\end{array}$ $\begin{array}{lll}1.14267000 & -0.07838900 & 0.17231300\end{array}$ $\begin{array}{lll}-3.22750400 & 3.66433200 & 3.49827600\end{array}$ $\begin{array}{llll}0.31034500 & 4.65004500 & 2.87644700\end{array}$ $\begin{array}{lll}-0.74427300 & 3.00553700 & 5.39980000\end{array}$ $\begin{array}{lll}-3.57557800 & -1.85567900 & 0.45920600\end{array}$ $-3.00662600-3.19584100-0.02222400$ $\begin{array}{lll}-4.23462000 & -2.02516000 & 1.83353700\end{array}$ $-4.60288300-1.32309100-0.54655000$ $\begin{array}{llll}-2.52231100 & -3.07499100 & -0.99630100\end{array}$ $\begin{array}{lll}-2.26069900 & -3.56994400 & 0.68664300\end{array}$ $\begin{array}{lll}-3.79281900 & -3.95228400 & -0.12119900\end{array}$ $\begin{array}{lll}-4.60242300 & -1.06023000 & 2.19657000\end{array}$ $\begin{array}{lll}-5.07986600 & -2.72047300 & 1.78628800\end{array}$ $\begin{array}{lll}-3.51531100 & -2.41421200 & 2.56094300\end{array}$ $-5.43809600-2.02052900-0.67370500$ $\begin{array}{lll}-5.00544900 & -0.36416500 & -0.20429500\end{array}$ $-4.13258400-1.16716300-1.52197100$ $2.46804300-0.36679300-0.21423400$ $\begin{array}{llll}3.04369300 & 0.84878800 & -0.95144500\end{array}$ $\begin{array}{llll}3.30491200 & -0.65139800 & 1.03859400\end{array}$ $2.46438100-1.59532900-1.13095100$ $\begin{array}{llll}2.44722400 & 1.07630300 & -1.83989300\end{array}$ $\begin{array}{llll}3.03020100 & 1.72637400 & -0.29661800\end{array}$ $\begin{array}{llll}4.07798100 & 0.67378600 & -1.26764000\end{array}$ $\begin{array}{llll}2.90663700 & -1.51395000 & 1.58189300\end{array}$ $\begin{array}{llll}4.34723300 & -0.86572600 & 0.77827900\end{array}$ $\begin{array}{lll}3.29101200 & 0.21465800 & 1.70770000\end{array}$ $3.47703200-1.85598200-1.45764200$ $\begin{array}{llll}2.04060000 & -2.45789400 & -0.60620800\end{array}$ $\begin{array}{lll}1.85546000 & -1.40231500 & -2.01946900\end{array}$ $\begin{array}{llll}-1.25205100 & 0.38278800 & -3.26788800\end{array}$ $\begin{array}{llll}-2.48467100 & 1.08713700 & -3.84774200\end{array}$ $\begin{array}{llll}0.01844100 & 0.92867200 & -3.93077600\end{array}$ $\begin{array}{lll}-1.35493200 & -1.12809500 & -3.51032000\end{array}$ $\begin{array}{llll}-3.39544500 & 0.72787200 & -3.35821200\end{array}$ $\begin{array}{llll}-2.41777300 & 2.16902100 & -3.69764000\end{array}$ $\begin{array}{lll}-2.57696900 & 0.89851700 & -4.92290900\end{array}$ $\begin{array}{llll}0.90342300 & 0.43212900 & -3.52197100\end{array}$ $\begin{array}{llll}0.00360900 & 0.76376500 & -5.01373100\end{array}$ $\begin{array}{lll}0.11529400 & 2.00421600 & -3.75256700\end{array}$ $\begin{array}{lll}0.1 .38984600 & -1.36040900 & -4.58040800\end{array}$ $-0.49124400-1.64183700-3.07588300$ $-2.26065300-1.52699100-3.04365000$ $\begin{array}{llll}-4.51933600 & 4.06114900 & 3.90314100\end{array}$ $\begin{array}{lll}-4.53898900 & 5.58305100 & 4.08971100\end{array}$ $\begin{array}{lll}-5.52257700 & 3.64685500 & 2.82011600\end{array}$ $\begin{array}{lll}-4.86159800 & 3.36429500 & 5.22430400\end{array}$ $\begin{array}{lll}-3.80522000 & 5.88103100 & 4.84558500\end{array}$ $\begin{array}{lll}-4.28695800 & 6.08763300 & 3.15153100\end{array}$ $\begin{array}{llll}-5.52537400 & 5.93417800 & 4.41186100\end{array}$ $\begin{array}{lll}-5.47790700 & 2.56517000 & 2.65800100\end{array}$ $\begin{array}{lll}-6.54708900 & 3.91038600 & 3.10472700\end{array}$ $\begin{array}{llll}-5.29419400 & 4.14574800 & 1.87314600\end{array}$ $\begin{array}{lll}-5.86666600 & 3.63144300 & 5.56856700\end{array}$ $\begin{array}{lll}-4.81673600 & 2.27726800 & 5.10263000\end{array}$ 


$\begin{array}{lrrr}\text { H } & -4.14503500 & 3.65195200 & 5.99924100 \\ \mathrm{C} & 1.29996200 & 5.65461100 & 2.88412700 \\ \mathrm{C} & 2.38789800 & 5.27188600 & 3.89345100 \\ \mathrm{C} & 1.90843400 & 5.76298900 & 1.48074500 \\ \mathrm{C} & 0.65609900 & 6.98803100 & 3.28344100 \\ \mathrm{H} & 1.95765600 & 5.18333700 & 4.89522200 \\ \mathrm{H} & 2.83211700 & 4.30790000 & 3.62481100 \\ \mathrm{H} & 3.18605300 & 6.02150200 & 3.92620900 \\ \mathrm{H} & 1.14560900 & 6.05153500 & 0.75094700 \\ \mathrm{H} & 2.70714200 & 6.51226000 & 1.45099500 \\ \mathrm{H} & 2.32924100 & 4.79964500 & 1.17565800 \\ \mathrm{H} & 1.39379100 & 7.79768500 & 3.30446800 \\ \mathrm{H} & -0.13035800 & 7.26144000 & 2.57259400 \\ \mathrm{H} & 0.20556400 & 6.90921600 & 4.27802300 \\ \mathrm{C} & -0.56197300 & 3.28244200 & 6.77336100 \\ \mathrm{C} & -1.49346600 & 2.38934000 & 7.60190200 \\ \mathrm{C} & 0.89550600 & 3.00554000 & 7.16279800 \\ \mathrm{C} & -0.89634600 & 4.75858300 & 7.02052300 \\ \mathrm{H} & -2.53605700 & 2.54857400 & 7.31191000 \\ \mathrm{H} & -1.25208100 & 1.33337700 & 7.44674700 \\ \mathrm{H} & -1.39739800 & 2.60484900 & 8.67175400 \\ \mathrm{H} & 1.57669900 & 3.60210100 & 6.54927700 \\ \mathrm{H} & 1.07509400 & 3.25147700 & 8.21508800 \\ \mathrm{H} & 1.14026700 & 1.94912600 & 7.01701600 \\ \mathrm{H} & -0.77499200 & 5.02749900 & 8.07562200 \\ \mathrm{H} & -0.23878000 & 5.40008100 & 6.42518300 \\ \mathrm{H} & -1.93131500 & 4.96543000 & 6.72986500 \\ & & & \\ & & & \end{array}$

\section{$\mathrm{Cat}_{\mathrm{B}}\left({ }^{\mathrm{B}} \mathrm{BuOH}\right)$}

$H=-969.20983 a u$

C $\quad 3.60420500 \quad 0.68016300 \quad-3.53170100$

$\mathrm{H} \quad 3.84232000 \quad 1.74852700 \quad-3.55049600$

H $\quad 4.36556800 \quad 0.14761100 \quad-4.11193600$

H $\quad 2.63594900 \quad 0.53629200 \quad-4.02221500$

C $\quad 3.17652800-1.31121600 \quad-2.05318400$

H $\quad 2.20383200-1.46980400 \quad-2.52993600$

H $\quad 3.92012500-1.92099900-2.57771700$

H $\quad 3.11225100-1.66299700 \quad-1.01829500$

C $\quad 4.89085900 \quad 0.40600100 \quad-1.38628100$

H $\quad 5.14153200 \quad 1.47170000 \quad-1.38906100$

$\begin{array}{lllll}\mathrm{H} & 4.83887100 & 0.06864800 & -0.34615200\end{array}$

H $\quad 5.70004400 \quad-0.13866300-1.88473800$

$\begin{array}{lllll}\text { C } & -2.19891800 & 0.60300400 & -2.82149900\end{array}$

$\mathrm{H} \quad-1.38353100-0.01557400 \quad-3.21034900$

H $\quad-3.14746700 \quad 0.17983200 \quad-3.16942900$

H $\quad-2.09671600 \quad 1.60884800 \quad-3.24155600$

C $\quad-3.25210700 \quad 1.55653500 \quad-0.74397300$

H $\quad-4.24276100 \quad 1.17200000 \quad-1.00974600$

H $\quad-3.18827300 \quad 1.61676200 \quad 0.34727500$

H $\quad-3.15614900 \quad 2.56886200 \quad-1.14944500$

C $\quad-2.25107100 \quad-0.75209300-0.70166400$

H $\quad-2.18110300-0.71261600 \quad 0.39014900$

$\mathrm{H} \quad-3.20361600-1.22248200 \quad-0.96848200$

H $\quad-1.43914500 \quad-1.38475400 \quad-1.07494200$

$\begin{array}{llll}\text { C } & 1.67591900 & 0.06620100 & 3.45711600\end{array}$

$\begin{array}{llll}\text { H } & 0.80534500 & -0.51019000 & 3.12775700\end{array}$

$\begin{array}{llll}\mathrm{H} & 2.55704000 & -0.31015500 & 2.92750000\end{array}$

H $\quad 1.81835600-0.10853400 \quad 4.52910000$

$\begin{array}{llll}\text { C } & 0.21793300 & 2.07656100 & 3.86553600\end{array}$

$\begin{array}{llll}\mathrm{H} & 0.05943700 & 3.13335400 & 3.62703100\end{array}$

$\begin{array}{llll}H & -0.66374200 & 1.51537800 & 3.53957700\end{array}$

$\begin{array}{llll}\mathrm{H} & 0.30460600 & 1.97954700 & 4.95317700\end{array}$

$\begin{array}{llll}\text { C } & 2.70231900 & 2.36042600 & 3.58315800\end{array}$ $\begin{array}{lll}2.88308800 & 2.27297900 & 4.65997800\end{array}$ $\begin{array}{lll}3.59209100 & 2.00212900 & 3.05528600\end{array}$ $\begin{array}{lll}2.56384300 & 3.41952500 & 3.34292800\end{array}$ $\begin{array}{llll}2.55253600 & 0.90066600 & -1.39725000\end{array}$ $\begin{array}{lll}1.29537100 & 1.70945700 & 1.76389700\end{array}$ $\begin{array}{lll}-0.89424400 & 1.20427900 & -0.91160900\end{array}$ $\begin{array}{llll}-2.13679200 & 0.65853800 & -1.29082800\end{array}$ $\begin{array}{lll}3.54616900 & 0.17615900 & -2.08518800\end{array}$ $\begin{array}{lll}1.47107800 & 1.55469900 & 3.15339700\end{array}$ $\begin{array}{llll}1.13770500 & 4.05553000 & -0.82732000\end{array}$ $1.21211800 \quad 5.42526900-1.14865800$ $0.40567500 \quad 6.21471400-0.11127700$ $\begin{array}{llll}2.68336800 & 5.85438700 & -1.11872900\end{array}$ $0.62485900 \quad 5.63162100-2.54958000$ $\begin{array}{lll}-0.64018500 & 5.89084300 & -0.11842800\end{array}$ $\begin{array}{lll}0.81078200 & 6.04411000 & 0.89137600\end{array}$ $\begin{array}{llll}0.43367200 & 7.29057600 & -0.31542500\end{array}$ $\begin{array}{llll}3.26224500 & 5.26944900 & -1.84071100\end{array}$ $\begin{array}{llll}2.79705300 & 6.91557400 & -1.36556600\end{array}$ $\begin{array}{lll}3.10622900 & 5.68588900 & -0.12297800\end{array}$ $\begin{array}{llll}0.65694800 & 6.68538900 & -2.84698100\end{array}$ $\begin{array}{llll}1.18867500 & 5.04979800 & -3.28603700\end{array}$ $\begin{array}{lll}-0.41787800 & 5.29905400 & -2.57491400\end{array}$ $\begin{array}{lll}1.02372100 & 1.97060300 & -0.34562500\end{array}$

\section{Int-1B ( $\left.{ }^{\mathrm{B} u O H}\right)$}

$H=-1353.533451 \mathrm{au}$

$\begin{array}{llll}\mathrm{N} & -0.59046600 & 2.40825700 & 0.32120900\end{array}$

$\begin{array}{llll}C & -1.07129400 & 2.59967500 & 1.45153600\end{array}$

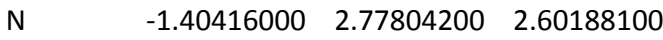

$\begin{array}{llll}\mathrm{O} & 0.95680600 & 0.07791200 & 1.87901200\end{array}$

$\begin{array}{llll}\text { C } & 1.61709600 & 0.15238100 & 3.11831600\end{array}$

$\begin{array}{llll}\text { C } & 0.63271200 & -0.24963000 & 4.22395200\end{array}$

H $\quad \begin{array}{llll}\mathrm{C} & 1.09793400 & -0.20634100 & 5.21510900\end{array}$

$\begin{array}{lllll}H & -0.22843400 & 0.42584500 & 4.21763100\end{array}$

H $\quad 0.27117200 \quad-1.26934500 \quad 4.05626900$

$\begin{array}{llll}\text { C } & 2.81491900 & -0.80551600 & 3.10594600\end{array}$

$\begin{array}{llll}\mathrm{H} & 3.51127200 & -0.52938900 & 2.30708300\end{array}$

H $\quad 3.35827700 \quad-0.78243100 \quad 4.05718800$

$\begin{array}{llll}H & 2.47840100 & -1.83129600 & 2.92678400\end{array}$

$\begin{array}{llll}\text { C } & 2.10192800 & 1.59050100 & 3.34251400\end{array}$

$\begin{array}{llll}\mathrm{H} & 1.25234500 & 2.27951500 & 3.35426400\end{array}$

$\begin{array}{llll}H & 2.63809700 & 1.68670700 & 4.29351700\end{array}$

$\begin{array}{llll}\mathrm{H} & 2.78009400 & 1.88904100 & 2.53619400\end{array}$

$\begin{array}{lllll}\text { U } & 0.21769600 & -0.24620700 & -0.12812200\end{array}$

O $\quad-1.93870300 \quad-0.50582300 \quad-0.36952700$

$\begin{array}{lllll}0 & 1.33341600 & 0.53257800 & -1.83944700\end{array}$

$\begin{array}{llll}\text { C } & -2.67949100 & 2.59757000 & 3.29388000\end{array}$

$\begin{array}{llll}\text { C } & -3.79555000 & 2.12839100 & 2.36718100\end{array}$

$\begin{array}{llll}\text { C } & -3.02222800 & 3.89318900 & 4.02605600\end{array}$

$\begin{array}{llll}\mathrm{H} & -2.48789900 & 1.82030400 & 4.04489300\end{array}$

H $\quad-3.51093800 \quad 1.21153300 \quad 1.84348500$

H $\quad-4.70619400 \quad 1.93137300 \quad 2.94116300$

$\begin{array}{llll}\mathrm{H} & -4.02579400 & 2.89529500 & 1.61932300\end{array}$

H $\quad-2.19954100 \quad 4.20251900 \quad 4.67580200$

$\begin{array}{llll}H & -3.21927800 & 4.69955800 & 3.31215700\end{array}$

$\begin{array}{llll}H & -3.91697000 & 3.75185100 & 4.63950000\end{array}$

C $\quad-0.56924000 \quad 3.53444400 \quad-0.65820600$

C $\quad-1.12207900 \quad 3.05222400 \quad-1.99274000$

$\begin{array}{lllll}\text { C } & 0.86038900 & 4.05315200 & -0.77061000\end{array}$

H $\quad-1.21242700 \quad 4.33746400 \quad-0.27346500$

H $\quad-2.14187400 \quad 2.67417800 \quad-1.88235900$

$\begin{array}{lllll}\mathrm{H} & -1.13463400 & 3.87843400 & -2.71092900\end{array}$ 
$\begin{array}{lll}-0.48934800 & 2.25522900 & -2.39538100\end{array}$ $\begin{array}{llll}1.22326600 & 4.42190700 & 0.19317500\end{array}$ $\begin{array}{llll}1.51977300 & 3.24998900 & -1.11050100\end{array}$ $\begin{array}{llll}0.90814700 & 4.87363600 & -1.49412800\end{array}$ $0.78159500-2.30627400-0.31978500$ $\begin{array}{llll}1.16640000 & -3.65684500 & -0.21927700\end{array}$ $0.56441300-4.43285900-1.39687300$ $2.69726500-3.73895200-0.26131400$ $\begin{array}{lll}0.64449500 & -4.22840400 & 1.10486200\end{array}$ $\begin{array}{lll}0.52826400 & -4.37409500 & -1.37082700\end{array}$ $\begin{array}{llll}0.90712500 & -4.01053800 & -2.34659800\end{array}$ $0.85117700-5.48989000-1.36684900$ $\begin{array}{llll}3.12906900 & -3.16144900 & 0.56201700\end{array}$ $3.04544300-4.77429700-0.17617700$ $3.07403600-3.32732200-1.20306800$ $\begin{array}{llll}0.91265400 & -5.28463200 & 1.21805400\end{array}$ $\begin{array}{lll}1.06491800 & -3.67495300 & 1.95018900\end{array}$ $\begin{array}{lll}-0.44604100 & -4.14398500 & 1.15023900\end{array}$ $\begin{array}{llll}2.31660100 & 0.39851000 & -2.84394100\end{array}$ $\begin{array}{llll}2.00299700 & -0.84289600 & -3.68849800\end{array}$ $\begin{array}{llll}2.29859000 & 1.64645600 & -3.73488700\end{array}$ $\begin{array}{llll}3.69114200 & 0.25195900 & -2.17818100\end{array}$ $\begin{array}{llll}1.97382200 & -1.73210100 & -3.05151500\end{array}$ $1.02467300-0.73522200-4.16863400$ $\begin{array}{llll}2.75378800 & -0.99980700 & -4.47100100\end{array}$ $\begin{array}{llll}2.52682400 & 2.54290900 & -3.15016900\end{array}$ $\begin{array}{llll}3.03768700 & 1.56811300 & -4.53985000\end{array}$ $\begin{array}{llll}1.31125400 & 1.77506000 & -4.18970600\end{array}$ $\begin{array}{llll}4.49338500 & 0.16021000 & -2.91884900\end{array}$ $\begin{array}{llll}3.90184700 & 1.12478800 & -1.55117800\end{array}$ $3.71089900-0.63983400-1.54266900$ $-3.04504800-1.13675300-0.97087900$ $\begin{array}{lll}-2.64393700 & -1.65479800 & -2.35919000\end{array}$ $\begin{array}{lll}-3.49381000 & -2.30447600 & -0.08289200\end{array}$ $-4.18424800-0.11919100-1.11098200$ $-2.31890600-0.82270200-2.99373000$ $\begin{array}{lll}-1.81531000 & -2.36519100 & -2.27297400\end{array}$ $-3.47577500-2.16049900-2.86216700$ $-3.75136100-1.93924800 \quad 0.91656100$ $\begin{array}{lll}-4.36825700 & -2.81695300 & -0.49948100\end{array}$ $\begin{array}{lll}-2.68372500 & -3.03342900 & 0.01933000\end{array}$ $-5.06278200-0.56514800-1.59055500$ $\begin{array}{lll}-4.48569800 & 0.25617000 & -0.12846300\end{array}$ $\begin{array}{lll}-3.85794200 & 0.73201200 & -1.71698100\end{array}$

\section{$\mathrm{TS}_{1 \mathrm{~B}-2 \mathrm{~B}}\left({ }^{\mathrm{t}} \mathrm{BuOH}\right)$}

$H=-1353.51314 a u$

$\begin{array}{llll}\mathrm{N} & -0.85908300 & 2.55393100 & 0.56279300\end{array}$

C $\quad-1.16335600 \quad 2.59371900 \quad 1.81816200$

$\begin{array}{lllll}\mathrm{N} & -1.76474100 & 3.17539400 & 2.71600700\end{array}$

$\begin{array}{lllll}\mathrm{O} & -0.21694900 & 1.01273300 & 2.35396700\end{array}$

C $\quad 0.93480800 \quad 1.06334300 \quad 3.21510900$

$\begin{array}{lllll}\text { C } & 0.69481000 & 0.08550200 & 4.36753700\end{array}$

$\begin{array}{llll}\mathrm{H} & 1.55226200 & 0.05534600 & 5.04880600\end{array}$

$\begin{array}{llll}\mathrm{H} & -0.18782900 & 0.38009900 & 4.94360600\end{array}$

$\begin{array}{llll}\text { H } & 0.52520600 & -0.92170200 & 3.97629700\end{array}$

$\begin{array}{lllll}\text { C } & 2.16741400 & 0.62912400 & 2.40663000\end{array}$

H $\quad 2.34330600 \quad 1.31657900 \quad 1.56858200$

$\begin{array}{llll}\mathrm{H} & 3.07329100 & 0.63814600 & 3.02145600\end{array}$

$\begin{array}{llll}\mathrm{H} & 2.05119200 & -0.39442600 & 2.02833900\end{array}$

C $\quad \begin{array}{llll}\text { C } & 1.17616600 & 2.47715500 & 3.74987100\end{array}$

$\begin{array}{lllll}\mathrm{H} & 0.34529500 & 2.82215300 & 4.36783600\end{array}$ H $\quad 2.08254900 \quad 2.48920300 \quad 4.36435300$ $\begin{array}{lll}1.31163700 & 3.18816800 & 2.92953800\end{array}$ $\begin{array}{lll}-0.21268300 & 0.10298300 & 0.17309400\end{array}$ $\begin{array}{lll}-2.14017300 & -0.28358200 & -0.68508700\end{array}$ $\begin{array}{llll}1.26228600 & 0.45805800 & -1.34396900\end{array}$ $\begin{array}{lll}-2.39068400 & 2.73084400 & 3.93987400\end{array}$ $\begin{array}{lll}-3.78037600 & 2.15673300 & 3.65047000\end{array}$ $-2.46050500 \quad 3.89319400 \quad 4.92816100$ $\begin{array}{lll}-1.77198100 & 1.92859800 & 4.36686300\end{array}$ $\begin{array}{lll}-3.71496000 & 1.30503300 & 2.96727700\end{array}$ $\begin{array}{lll}-4.25564000 & 1.81780700 & 4.57718200\end{array}$ $\begin{array}{lll}-4.42106300 & 2.91721000 & 3.19248600\end{array}$ $\begin{array}{lll}-1.46459400 & 4.29538200 & 5.13531600\end{array}$ $\begin{array}{lll}-3.07201000 & 4.70425800 & 4.51954800\end{array}$ $\begin{array}{lll}-2.90576200 & 3.56780200 & 5.87356100\end{array}$ $\begin{array}{llll}-1.54782300 & 3.55861700 & -0.28144600\end{array}$ $\begin{array}{llll}-1.44098900 & 3.13249600 & -1.74013100\end{array}$ $\begin{array}{llll}-0.94431900 & 4.94425100 & -0.05409900\end{array}$ $\begin{array}{lll}-2.60964700 & 3.59053500 & 0.00519400\end{array}$ $\begin{array}{lll}-1.90181800 & 2.15259600 & -1.89723300\end{array}$ $\begin{array}{llll}-1.94607900 & 3.85527800 & -2.38885200\end{array}$ $\begin{array}{llll}-0.39089000 & 3.07414200 & -2.04577000\end{array}$ $\begin{array}{lll}-1.03012500 & 5.23850200 & 0.99559400\end{array}$ $\begin{array}{llll}0.11499300 & 4.94776100 & -0.33244100\end{array}$ $\begin{array}{llll}-1.46251000 & 5.69364400 & -0.66233600\end{array}$ $\begin{array}{llll}0.30339200 & -1.89460500 & 0.80355500\end{array}$ $\begin{array}{lll}0.53426600 & -3.25798700 & 1.07225600\end{array}$ $0.36842000-4.04757700-0.23211600$ $\begin{array}{llll}1.96104500 & -3.42718700 & 1.60824900\end{array}$ $\begin{array}{lll}-0.48182400 & -3.74063500 & 2.11418900\end{array}$ $-0.64144200-3.90947600-0.63131600$ $\begin{array}{llll}1.08339100 & -3.69323000 & -0.98155400\end{array}$ $\begin{array}{llll}0.53356300 & -5.11962900 & -0.07811000\end{array}$ $\begin{array}{lll}2.08657800 & -2.87184500 & 2.54345200\end{array}$ $\begin{array}{llll}2.19154000 & -4.47995700 & 1.80490400\end{array}$ $\begin{array}{llll}2.68562500 & -3.04635700 & 0.88151700\end{array}$ $\begin{array}{lll}-0.34637800 & -4.80350700 & 2.34282000\end{array}$ $\begin{array}{lll}-0.37290000 & -3.17371400 & 3.04422800\end{array}$ $\begin{array}{lll}-1.50140800 & -3.59491800 & 1.74442300\end{array}$ $\begin{array}{lll}2.24496200 & 0.51780600 & -2.35175100\end{array}$ $\begin{array}{llll}1.55834500 & 0.38338000 & -3.71591400\end{array}$ $\begin{array}{llll}2.96763700 & 1.86630100 & -2.25146600\end{array}$ $3.23943000-0.63013700-2.14374200$ $\begin{array}{llll}1.02599100 & -0.57114000 & -3.77905100\end{array}$ $\begin{array}{llll}0.83113300 & 1.18939400 & -3.85537000\end{array}$ $\begin{array}{llll}2.28382500 & 0.42774000 & -4.53558700\end{array}$ $\begin{array}{llll}3.44311000 & 1.96979800 & -1.27076200\end{array}$ $\begin{array}{llll}3.74185500 & 1.96344700 & -3.02041500\end{array}$ $\begin{array}{lll}2.25621300 & 2.68891300 & -2.37354300\end{array}$ $\begin{array}{llll}4.02551200 & -0.62082300 & -2.90668700\end{array}$ $\begin{array}{llll}3.71361700 & -0.54703400 & -1.16048900\end{array}$ $\begin{array}{llll}2.72245100 & -1.59356700 & -2.19397000\end{array}$ $\begin{array}{lll}2.3 .33052500 & -0.83618600 & -1.20224800\end{array}$ $\begin{array}{llll}-3.05768700 & -1.36309400 & -2.61571700\end{array}$ $\begin{array}{lll}-3.78313100 & -1.97994200 & -0.28728600\end{array}$ $\begin{array}{llll}-4.40396800 & 0.25773000 & -1.24114800\end{array}$ $\begin{array}{lll}-2.70561900 & -0.55204300 & -3.26094500\end{array}$ $\begin{array}{lll}-2.28482700 & -2.13821500 & -2.58897300\end{array}$ $\begin{array}{llll}-3.95983200 & -1.79329000 & -3.06428100\end{array}$ $\begin{array}{llll}-3.95625200 & -1.60679700 & 0.72748500\end{array}$ $\begin{array}{llll}-4.71036100 & -2.43840600 & -0.64792700\end{array}$ $\begin{array}{lll}-3.01206400 & -2.75499100 & -0.24037300\end{array}$ $\begin{array}{lll}-5.35620000 & -0.13435300 & -1.61476800\end{array}$ $\begin{array}{llll}-4.56850300 & 0.66258400 & -0.23773000\end{array}$ 


\section{Int-2B (' $\left.{ }^{\mathrm{B} u O H}\right)$}

$H=-1353.525228 a u$

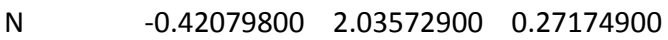

$\begin{array}{llll}\text { C } & -0.71924100 & 1.85838000 & 1.57900000\end{array}$

$\begin{array}{llll}\mathrm{N} & -1.56632000 & 2.48499200 & 2.29310500\end{array}$

$\begin{array}{lllll}\text { O } & 0.01682300 & 0.69734900 & 2.03153600\end{array}$

$\begin{array}{llll}\text { C } & 1.32241000 & 0.90200600 & 2.70421100\end{array}$

$\begin{array}{llll}\text { C } & 1.35466700 & -0.13493000 & 3.81981200\end{array}$

$\begin{array}{llll}\mathrm{H} & 2.32157600 & -0.11382400 & 4.33270200\end{array}$

$\begin{array}{llll}\mathrm{H} & 0.56972000 & 0.06147900 & 4.55498800\end{array}$

$\begin{array}{llll}\mathrm{H} & 1.20119000 & -1.13513700 & 3.40777200\end{array}$

$\begin{array}{llll}\text { C } & 2.42518200 & 0.63073300 & 1.67906800\end{array}$

$\begin{array}{llll}\mathrm{H} & 2.37508000 & 1.35207300 & 0.85426100\end{array}$

$\begin{array}{llll}\mathrm{H} & 3.41371000 & 0.73804100 & 2.13597000\end{array}$

H $\quad 2.36537900 \quad-0.39977400 \quad 1.30525300$

$\begin{array}{llll}\text { C } & 1.46512100 & 2.31747900 & 3.25096000\end{array}$

$\begin{array}{llll}\mathrm{H} & 0.70020500 & 2.54555700 & 3.99436000\end{array}$

$\begin{array}{llll}\mathrm{H} & 2.44370000 & 2.40430100 & 3.73377200\end{array}$

$\begin{array}{llll}\mathrm{H} & 1.41336000 & 3.06272200 & 2.45298300\end{array}$

U $\quad 0.12432800 \quad-0.21137200 \quad-0.48243700$

$\begin{array}{lllll}0 & -1.68709700 & -0.72152500 & -1.47286600\end{array}$

$\begin{array}{lllll}0 & 1.59035200 & 0.07050700 & -2.00612900\end{array}$

C $\quad-1.94829400 \quad 2.07890300 \quad 3.62988600$

C $\quad-3.27736500 \quad 1.31740900 \quad 3.56378900$

C $\quad-2.08277900 \quad 3.32166800 \quad 4.51243700$

$\begin{array}{llll}\text { H } & -1.20654100 & 1.40273000 & 4.08432200\end{array}$

$\begin{array}{llll}H & -3.17013000 & 0.40001300 & 2.97649800\end{array}$

H $\quad-3.62481300 \quad 1.04378800 \quad 4.56652200$

$\begin{array}{llll}\text { H } & -4.04321200 & 1.93795200 & 3.08710300\end{array}$

H $\quad-1.13798000 \quad 3.87165300 \quad 4.57159600$

H $\quad-2.83186200 \quad 3.99976500 \quad 4.09054700$

$\begin{array}{llll}\mathrm{H} & -2.38886600 & 3.05341800 & 5.52953300\end{array}$

C $\quad-1.07578400 \quad 3.15950500 \quad-0.40084800$

C $\quad-\quad \begin{array}{llll}\text { C } & -99605700 & 2.96240600 & -1.91240800\end{array}$

$\begin{array}{llll}\text { C } & -0.44533000 & 4.49028500 & 0.01717600\end{array}$

H $\quad-2.13302200 \quad 3.18153700 \quad-0.09609500$

$\begin{array}{llll}H & -1.49849700 & 2.03854400 & -2.22129100\end{array}$

H $\quad-1.48034300 \quad 3.79109200 \quad-2.43890300$

H $\quad 0.04794400 \quad 2.92010200 \quad-2.24561100$

$\begin{array}{lllll}H & -0.52893800 & 4.62175400 & 1.09888000\end{array}$

$\begin{array}{lllll}H & 0.61319300 & 4.51868000 & -0.26642400\end{array}$

H $\quad-0.95252300 \quad 5.33164000 \quad-0.46850400$

$\begin{array}{llll}0 & 0.67328500 & -2.09119000 & 0.43538800\end{array}$

$\begin{array}{llll}\text { C } & 0.83318700 & -3.42392100 & 0.86660500\end{array}$

$\begin{array}{lllll}\text { C } & 0.63910700 & -4.35542800 & -0.33665300\end{array}$

$\begin{array}{llll}C & 2.24758900 & -3.59864600 & 1.43314400\end{array}$

C $\quad-0.21441500 \quad-3.73283500 \quad 1.94283600$

$\mathrm{H} \quad-0.36169700 \quad-4.22265000 \quad-0.75994400$

H $\quad 1.37442500-4.12647700 \quad-1.11469500$

H $\quad 0.75414100 \quad-5.40754800 \quad-0.05358500$

$\begin{array}{lllll}\mathrm{H} & 2.40288500 & -2.94198600 & 2.29512800\end{array}$

H $\quad 2.42279800-4.63032200 \quad 1.75705400$

$\begin{array}{llll}\mathrm{H} & 2.99254500 & -3.34696300 & 0.67145900\end{array}$

$\begin{array}{llll}\mathrm{H} & -0.12523000 & -4.76300200 & 2.30471600\end{array}$

$\begin{array}{llll}H & -0.09848800 & -3.05889300 & 2.79718300\end{array}$

$\begin{array}{llll}H & -1.22304300 & -3.59735500 & 1.53942500\end{array}$

$\begin{array}{lllll}\text { C } & 2.52816600 & 0.04101200 & -3.06096700\end{array}$

$\begin{array}{lllll}C & & 1.77497500 & 0.17957800 & -4.38824100\end{array}$

$\begin{array}{lllll}\text { C } & 3.50024500 & 1.21118200 & -2.87587600\end{array}$

C $\quad 3.28327300 \quad-1.29158400 \quad-3.01309800$

H $\quad 1.06843300 \quad-0.64732700-4.51175100$ $\begin{array}{lll}1.21135000 & 1.11740800 & -4.40808000\end{array}$

$\begin{array}{lll}2.46405500 & 0.17292300 & -5.23967100\end{array}$

$\begin{array}{llll}4.02832900 & 1.11944200 & -1.92127300\end{array}$

$\begin{array}{llll}4.24509100 & 1.24003700 & -3.67837800\end{array}$

$\begin{array}{llll}2.95541000 & 2.16031700 & -2.87664100\end{array}$

$\begin{array}{llll}4.03328800 & -1.35410100 & -3.80897400\end{array}$

$\begin{array}{llll}3.79257600 & -1.40502300 & -2.05073300\end{array}$

$2.58640100-2.12746100-3.13206800$

$-2.84072100-1.28595000-2.06291000$

$\begin{array}{llll}-2.41277800 & -2.11372700 & -3.27877600\end{array}$

$-3.53274500-2.17578300-1.02493000$

$-3.77369400-0.14931500-2.49241700$

$\begin{array}{llll}-1.90070700 & -1.47788000 & -4.00777900\end{array}$

$\begin{array}{lll}-1.72514300 & -2.90919400 & -2.97396100\end{array}$

$\begin{array}{lll}-3.27618200 & -2.57470000 & -3.77057000\end{array}$

$\begin{array}{lll}-3.81742800 & -1.58506500 & -0.14816300\end{array}$

$-4.43705000-2.63803500-1.43498700$

$\begin{array}{lll}-2.85782500 & -2.97331400 & -0.69808200\end{array}$

$\begin{array}{lll}-4.69391200 & -0.54052800 & -2.93946900\end{array}$

$\begin{array}{llll}-4.04414000 & 0.46627200 & -1.62906500\end{array}$

$\begin{array}{lll}-3.28023300 & 0.49051800 & -3.23051100\end{array}$

\section{Int-3B ('BuOH)}

$H=-1586.995027 \mathrm{au}$

$\begin{array}{llll}\mathrm{N} & -0.55496900 & 0.73322500 & 1.82208900\end{array}$

$\begin{array}{llll}\text { C } & 0.23768200 & -0.08405000 & 2.56641400\end{array}$

N $\quad 0.00711200-0.54958400 \quad 3.72888100$

$\begin{array}{lllll}\text { O } & 1.36507600 & -0.43757300 & 1.72990300\end{array}$

$\begin{array}{llll}\text { C } & 2.71815000 & 0.09447200 & 2.01070900\end{array}$

$\begin{array}{llll}\text { C } & 3.57997700 & -1.01021700 & 2.62084500\end{array}$

$\begin{array}{llll}\mathrm{H} & 4.63139900 & -0.70500100 & 2.60393800\end{array}$

H $\quad 3.31403800 \quad-1.22024100 \quad 3.65749900$

H $\quad 3.48294700 \quad-1.93087500 \quad 2.03842200$

$\begin{array}{llll}\text { C } & 3.28950700 & 0.47803400 & 0.64889100\end{array}$

$\begin{array}{llll}\mathrm{H} & 2.70931300 & 1.28470500 & 0.19294900\end{array}$

$\begin{array}{llll}\mathrm{H} & 4.32062400 & 0.82797200 & 0.76101700\end{array}$

$\begin{array}{llll}\text { H } & 3.29868000 & -0.37996800 & -0.02908600\end{array}$

$\begin{array}{llll}\text { C } & 2.65246500 & 1.31946000 & 2.91705800\end{array}$

$\begin{array}{llll}\mathrm{H} & 2.18673500 & 1.08791500 & 3.87685500\end{array}$

$\begin{array}{llll}\mathrm{H} & 3.66856900 & 1.68023700 & 3.10456800\end{array}$

$\begin{array}{llll}\mathrm{H} & 2.08725400 & 2.12595700 & 2.44397400\end{array}$

U $\quad-0.12844100-0.09061300-0.53307600$

O $\quad-1.91900000 \quad 0.56104600 \quad-1.50727800$

$\begin{array}{lllll}0 & 1.34584900 & -0.15907900 & -2.10992900\end{array}$

C $\quad 0.70934800 \quad-1.64845300 \quad 4.35606700$

$\begin{array}{lllll}\text { C } & -0.30056500 & -2.76957400 & 4.63084100\end{array}$

C $\quad 1.34586100 \quad-1.19283200 \quad 5.67265000$

$\mathrm{H} \quad 1.48833900-2.06126500 \quad 3.70092900$

H $\quad-0.76188200 \quad-3.11363200 \quad 3.70077300$

H $\quad 0.18622200 \quad-3.62644100 \quad 5.11044600$

H $\quad-1.09726600 \quad-2.40931800 \quad 5.29018400$

H $\quad 2.11391900-0.42898700 \quad 5.51517000$

$\begin{array}{llll}H & 0.57901700 & -0.76127900 & 6.32449500\end{array}$

$\begin{array}{llll}\mathrm{H} & 1.81003800 & -2.03555600 & 6.19682300\end{array}$

$\begin{array}{llll}\text { C } & -1.87262600 & 1.12955600 & 2.35194100\end{array}$

$\begin{array}{llll}\text { C } & -1.75974000 & 2.01727700 & 3.59593800\end{array}$

$\begin{array}{llll}\text { C } & -2.82913900 & -0.04487200 & 2.58514100\end{array}$

$\begin{array}{llll}\text { H } & -2.31201500 & 1.74286700 & 1.55175900\end{array}$

$\begin{array}{llll}H & -1.11390300 & 2.88146900 & 3.40202000\end{array}$

$\begin{array}{llll}H & -2.74629700 & 2.39197900 & 3.89072600\end{array}$

$\begin{array}{llll}\mathrm{H} & -1.33439200 & 1.44957900 & 4.42629900\end{array}$

H $\quad-2.91915400 \quad-0.65963000 \quad 1.68285100$

H $\quad-2.47199900 \quad-0.67694400 \quad 3.40010300$ 
$\begin{array}{llll}H & -3.82815800 & 0.32674700 & 2.83939600\end{array}$

$\begin{array}{llll}\mathrm{H} & 0.13307200 & 2.18233200 & 0.93677200\end{array}$

$\begin{array}{lllll}\text { C } & 0.33116300 & 3.79681400 & -0.33195500\end{array}$

$\begin{array}{lllll}\text { C } & 1.25653000 & 4.61906400 & 0.56547500\end{array}$

$\begin{array}{llll}\mathrm{H} & 1.20118600 & 5.68194400 & 0.30923700\end{array}$

$\begin{array}{llll}\mathrm{H} & 0.97404700 & 4.51446300 & 1.61866700\end{array}$

$\begin{array}{lllll}\mathrm{H} & 2.29374900 & 4.29086800 & 0.45111900\end{array}$

C $\quad-1.12670500 \quad 4.21752800 \quad-0.15159400$

H $\quad-1.27205300 \quad 5.25465600 \quad-0.47037800$

$\begin{array}{lllll}H & -1.78090500 & 3.57406300 & -0.74596500\end{array}$

$\begin{array}{llll}\mathrm{H} & -1.43059700 & 4.14423100 & 0.89829600\end{array}$

C $\quad 0.75421300 \quad 3.90144800 \quad-1.79242300$

H $\quad 1.78072100 \quad 3.54704000 \quad-1.92026400$

H $\quad 0.09834800 \quad 3.29978200 \quad-2.42820500$

H $\quad 0.70229600 \quad 4.94076000 \quad-2.13030300$

$\begin{array}{lllll}0 & 0.47185000 & 2.40238500 & 0.02410100\end{array}$

$\begin{array}{lllll}0 & -0.57548900 & -2.16552200 & -0.33313600\end{array}$

$\begin{array}{llll}\text { C } & -0.80806800 & -3.55529900 & -0.29951400\end{array}$

$\begin{array}{lllll}\text { C } & 0.35267000 & -4.22553300 & 0.44403400\end{array}$

$\begin{array}{llll}\text { C } & -2.13034100 & -3.81475500 & 0.43045400\end{array}$

$\begin{array}{llll}\text { C } & -0.88695400 & -4.07782600 & -1.73837000\end{array}$

H $\quad \begin{array}{llll}\text { H } & 1.29669900 & -4.02647900 & -0.07347600\end{array}$

H $\quad 0.42966900 \quad-3.82828100 \quad 1.46031100$

$\begin{array}{llll}\mathrm{H} & 0.21497700 & -5.31071100 & 0.50431500\end{array}$

H $\quad-2.95554300 \quad-3.32261400 \quad-0.09406900$

H $\quad-2.35059200 \quad-4.88632800 \quad 0.48793900$

H $\quad-2.08681400 \quad-3.41533800 \quad 1.44811100$

H $\quad-1.06866700 \quad-5.15798100 \quad-1.76042700$

H $\quad-1.69873400-3.58093100-2.27832500$

$\begin{array}{lllll}\mathrm{H} & 0.05026300 & -3.87669600 & -2.26704500\end{array}$

C $\quad 2.18680400 \quad-0.37264100-3.21884500$

$\begin{array}{lllll}\text { C } & 3.14138300 & 0.81866800 & -3.36427700\end{array}$

$\begin{array}{llll}\text { C } & 2.98573300 & -1.66366500 & -2.99938400\end{array}$

C $\quad 1.31871900 \quad-0.50342900-4.47662700$

$\begin{array}{lllll}\mathrm{H} & 2.57335700 & 1.74043600 & -3.52281200\end{array}$

$\begin{array}{llll}\mathrm{H} & 3.74343700 & 0.93747400 & -2.45805600\end{array}$

H $\quad 3.82141300 \quad 0.68499400 \quad-4.21289000$

$\begin{array}{llll}H & 2.30526200 & -2.50934300 & -2.86008200\end{array}$

H $\quad 3.63658800 \quad-1.88189600 \quad-3.85324800$

H $\quad 3.61387100 \quad-1.58025100-2.10650900$

H $\quad 1.92982400-0.66203900 \quad-5.37219400$

H $\quad 0.63038400 \quad-1.34864000 \quad-4.37550000$

H $\quad 0.72657100 \quad 0.40580000 \quad-4.62144700$

C $\quad-3.09288800 \quad 0.66916200 \quad-2.28296200$

C $\quad-3.46742300 \quad-0.71620700 \quad-2.82106100$

C $\quad-4.21926100 \quad 1.21079200 \quad-1.39587200$

$\begin{array}{lllll}\text { C } & -2.82508900 & 1.62961600 & -3.44769400\end{array}$

$\mathrm{H} \quad-2.66253000-1.11042800-3.44965600$

H $\quad-3.62780400-1.41218500 \quad-1.99204900$

H $\quad-4.38325200 \quad-0.67652500 \quad-3.42084700$

$\begin{array}{llll}\mathrm{H} & -3.94869300 & 2.19080200 & -0.99039700\end{array}$

$\mathrm{H} \quad-5.15221400 \quad 1.31910400 \quad-1.95967800$

H $\quad-4.40058100 \quad 0.53240600 \quad-0.55670500$

$\begin{array}{llll}\text { H } & -3.70905600 & 1.73877900 & -4.08546400\end{array}$

H $\quad-2.55168700 \quad 2.62076600 \quad-3.07171900$

H $\quad-2.00091500 \quad 1.25625500 \quad-4.06352000$

\section{$\mathrm{TS}_{3 \mathrm{~B}-4 \mathrm{~B}}$ ( $\left.{ }^{\mathrm{B}} \mathrm{BuOH}\right)$}

$H=-1586.996363 a u$

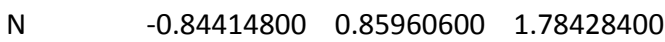

$\begin{array}{lllll}\text { C } & -0.03101400 & 0.01407100 & 2.49187000\end{array}$

N $\quad \begin{array}{llll}\mathrm{-} & -0.27203300 & -0.48691700 & 3.63343600\end{array}$

$\begin{array}{llll}\text { O } & 1.07444700 & -0.31164600 & 1.63562600\end{array}$ $\begin{array}{lll}2.44808000 & 0.17756800 & 1.91735000\end{array}$

$\begin{array}{lll}3.28225100 & -0.95835300 & 2.50598800\end{array}$

$\begin{array}{lll}4.34085900 & -0.67962200 & 2.48421200\end{array}$

$\begin{array}{lll}3.02024600 & -1.17485400 & 3.54224000\end{array}$

$\begin{array}{lll}3.15830600 & -1.86828800 & 1.91194200\end{array}$

$3.01710800 \quad 0.56493800 \quad 0.55556000$

$\begin{array}{llll}2.44034200 & 1.38118300 & 0.11340000\end{array}$

$\begin{array}{lll}4.05326000 & 0.89921900 & 0.66774600\end{array}$

$\begin{array}{llll}3.01565700 & -0.28831400 & -0.12955600\end{array}$

$\begin{array}{llll}2.40943500 & 1.39006300 & 2.83988100\end{array}$

$\begin{array}{lll}1.94848200 & 1.15233500 & 3.80089100\end{array}$

$\begin{array}{lll}3.43249700 & 1.73135700 & 3.02578500\end{array}$

$\begin{array}{lll}1.85428700 & 2.20978000 & 2.37859400\end{array}$

$\begin{array}{lll}-0.38015300 & -0.04665100 & -0.67772100\end{array}$

$\begin{array}{llll}-2.16376700 & 0.55860000 & -1.68062600\end{array}$

$\begin{array}{lll}1.06777700 & -0.17960700 & -2.25641300\end{array}$

$\begin{array}{llll}0.42034500 & -1.60689000 & 4.23587200\end{array}$

$\begin{array}{lll}-0.60680600 & -2.71137600 & 4.51076500\end{array}$

$\begin{array}{llll}1.08038300 & -1.17692900 & 5.54934900\end{array}$

$\begin{array}{lll}1.18359200 & -2.02241000 & 3.56435600\end{array}$

$\begin{array}{lll}1.1 .09054200 & -3.03368300 & 3.58455100\end{array}$

$\begin{array}{lll}-0.12741000 & -3.58370200 & 4.96884400\end{array}$

$-1.38436400-2.34626900 \quad 5.18984600$

$\begin{array}{lll}1.85585100 & -0.42057600 & 5.39298300\end{array}$

$\begin{array}{lll}0.32817600 & -0.74644600 & 6.21852000\end{array}$

$\begin{array}{llll}1.54065400 & -2.03373300 & 6.05333900\end{array}$

$\begin{array}{lll}-2.16847900 & 1.22398600 & 2.32102600\end{array}$

$\begin{array}{lll}-2.07524100 & 2.06309300 & 3.59899700\end{array}$

$\begin{array}{lll}-3.10680900 & 0.02729200 & 2.49223000\end{array}$

$\begin{array}{lll}-2.60180900 & 1.86288700 & 1.53886500\end{array}$

$\begin{array}{lll}-1.43889900 & 2.94106200 & 3.44311900\end{array}$

$\begin{array}{lll}-3.06940800 & 2.41438900 & 3.89641300\end{array}$

$\begin{array}{lll}-1.65290200 & 1.46928500 & 4.41225900\end{array}$

$\begin{array}{lll}-3.16598300 & -0.55771500 & 1.56803900\end{array}$

$\begin{array}{lll}-2.76000900 & -0.62636100 & 3.29453800\end{array}$

$\begin{array}{lll}-4.11692600 & 0.37482100 & 2.73470400\end{array}$

$\begin{array}{llll}-0.27835300 & 1.85146500 & 1.03906400\end{array}$

$\begin{array}{llll}0.05307400 & 3.65133500 & -0.30351900\end{array}$

$\begin{array}{lll}1.02851700 & 4.45345000 & 0.56245600\end{array}$

$\begin{array}{llll}1.00001900 & 5.51774300 & 0.30552400\end{array}$

$\begin{array}{llll}0.77324100 & 4.35700800 & 1.62312600\end{array}$

$\begin{array}{llll}2.05221400 & 4.09432200 & 0.41941300\end{array}$

$\begin{array}{llll}-1.38069800 & 4.14076100 & -0.08153300\end{array}$

$\begin{array}{lll}-1.49123600 & 5.18483700 & -0.39335900\end{array}$

$\begin{array}{llll}-2.08212400 & 3.53153300 & -0.65915700\end{array}$

$\begin{array}{llll}-1.65562800 & 4.07639900 & 0.97645400\end{array}$

$\begin{array}{llll}0.43124800 & 3.76830300 & -1.77983900\end{array}$

$\begin{array}{llll}1.44146300 & 3.38251700 & -1.94562500\end{array}$

$\begin{array}{llll}-0.26351700 & 3.19022800 & -2.39736200\end{array}$

$\begin{array}{llll}0.40020800 & 4.81085000 & -2.11294500\end{array}$

$\begin{array}{lll}0.15170800 & 2.26870300 & 0.04924600\end{array}$

$\begin{array}{lll}-0.83003700 & -2.12280200 & -0.39728600\end{array}$

$\begin{array}{lll}-1.08757800 & -3.50596500 & -0.32761000\end{array}$

$\begin{array}{llll}0.04660500 & -4.17807200 & 0.45459900\end{array}$

$\begin{array}{llll}-2.42873700 & -3.72386000 & 0.38144400\end{array}$

$\begin{array}{lll}-1.14747600 & -4.06707000 & -1.75331500\end{array}$

$\begin{array}{llll}1.00339100 & -4.00824800 & -0.04985300\end{array}$

$\begin{array}{llll}0.11241100 & -3.75802000 & 1.46244700\end{array}$

$\begin{array}{llll}-0.11096300 & -5.25895600 & 0.53902300\end{array}$

$\begin{array}{lll}-3.23375000 & -3.23046300 & -0.17272100\end{array}$

$\begin{array}{lll}-2.67012800 & -4.78937000 & 0.46244200\end{array}$

$\begin{array}{lll}-2.39917600 & -3.29864100 & 1.38917600\end{array}$

$\begin{array}{lll}-1.34722200 & -5.14426500 & -1.75058600\end{array}$ 


\begin{tabular}{|c|c|c|c|}
\hline t & -1.93900500 & -3.57118900 & -2.32352200 \\
\hline & -0.19672900 & -3.89528500 & -2.26804500 \\
\hline & 1.88767300 & -0.42095900 & -3.37728200 \\
\hline & 2.81792500 & 0.78049800 & -3.58007700 \\
\hline & 2.70996300 & -1.69080700 & -3.12670900 \\
\hline 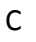 & 0.99445300 & -0.60843400 & -4.60931200 \\
\hline$y$ & 2.23105200 & 1.68815300 & -3.75074100 \\
\hline 1 & 3.43909300 & 0.93527800 & -2.69222200 \\
\hline 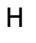 & 3.47949000 & 0.63182100 & -4.44056500 \\
\hline$y$ & 2.04555100 & -2.54175500 & -2.94756300 \\
\hline 1 & 3.35042700 & -1.92768100 & -3.98332100 \\
\hline 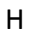 & 3.35044800 & -1.56610700 & -2.24749200 \\
\hline 1 & 1.58942300 & -0.78865100 & -5.51147600 \\
\hline $\mid$ & 0.32296500 & -1.46065900 & -4.46413000 \\
\hline & 0.38501300 & 0.28596400 & -4.77273900 \\
\hline & -3.33634700 & 0.66854800 & -2.45873800 \\
\hline 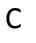 & -3.57222700 & -0.65454800 & -3.19527600 \\
\hline 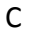 & -4.51519300 & 0.97258300 & -1.52772300 \\
\hline 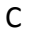 & -3.14952400 & 1.80676900 & -3.46825800 \\
\hline 1 & -2.72216600 & -0.88322100 & -3.84592600 \\
\hline $\boldsymbol{\gamma}$ & -3.68525000 & -1.47243500 & -2.47703600 \\
\hline 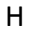 & -4.47604200 & -0.61113800 & -3.81279100 \\
\hline+ & -4.33927300 & 1.90503900 & -0.98214900 \\
\hline 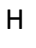 & -5.44991300 & 1.07515800 & -2.08972500 \\
\hline$H$ & -4.64015700 & 0.16723400 & -0.79726600 \\
\hline $\mathrm{H}$ & -4.03543700 & 1.92553000 & -4.10147900 \\
\hline $\mathrm{H}$ & -2.97107100 & 2.75355500 & -2.94903700 \\
\hline & -2.29002700 & 1.60209200 & -4.11432200 \\
\hline
\end{tabular}

\section{Int-4B ('BuOH)}

\section{$H=-1587.003605 a u$}

$\begin{array}{llll}\mathrm{N} & -0.63475700 & 0.77987600 & 2.05071500\end{array}$

$\begin{array}{llll}\text { C } & 0.25349100 & -0.10240400 & 2.66396700\end{array}$

$\begin{array}{llll}\mathrm{N} & -0.01107400 & -0.70732800 & 3.74053300\end{array}$

$\begin{array}{lllll}\text { O } & 1.34042500 & -0.27583600 & 1.79309500\end{array}$

$\begin{array}{lllll}\text { C } & 2.69452000 & 0.26416700 & 2.10011100\end{array}$

$\begin{array}{llll}\text { C } & 3.56316400 & -0.84332500 & 2.68855900\end{array}$

$\begin{array}{llll}\mathrm{H} & 4.60786800 & -0.51609600 & 2.69411400\end{array}$

$\begin{array}{lllll}H & 3.29006900 & -1.08981100 & 3.71518000\end{array}$

$\begin{array}{llll}\text { H } & 3.49363000 & -1.74633300 & 2.07573300\end{array}$

$\begin{array}{lllll}\text { C } & 3.24724700 & 0.68235000 & 0.74220300\end{array}$

$\begin{array}{lllll}\mathrm{H} & 2.62105700 & 1.46170000 & 0.29860900\end{array}$

$\begin{array}{llll}\mathrm{H} & 4.26128000 & 1.07673000 & 0.85992400\end{array}$

$\begin{array}{llll}\text { H } & 3.30000200 & -0.17272400 & 0.06108100\end{array}$

$\begin{array}{llll}\text { C } & 2.58731300 & 1.46585900 & 3.03079800\end{array}$

$\begin{array}{llll}\mathrm{H} & 2.11274600 & 1.20359100 & 3.98009300\end{array}$

H $\quad 3.59146200 \quad 1.84165600 \quad 3.24901900$

$\begin{array}{llll}\mathrm{H} & 2.02098700 & 2.27270000 & 2.55875000\end{array}$

U $\quad-0.09091200 \quad 0.12570100-0.68601000$

$\begin{array}{lllll}0 & -1.86534000 & 0.65297100 & -1.74585800\end{array}$

$\begin{array}{lllll}0 & 1.36469700 & -0.13794800 & -2.23225500\end{array}$

C $\quad 0.72738900 \quad-1.82152800 \quad 4.30208300$

C $\quad-0.25317100 \quad-2.97315300 \quad 4.54372100$

C $\quad 1.36351600 \quad-1.39734000 \quad 5.62927000$

H $\quad 1.50962000-2.17728400 \quad 3.61802200$

$\begin{array}{llll}\mathrm{H} & -0.72309700 & -3.29397300 & 3.61084500\end{array}$

H $\quad 0.26599600 \quad-3.83414000 \quad 4.97813900$

H $\quad-1.04337400 \quad-2.66011000 \quad 5.23378500$

H $\quad 2.08149500-0.58148500 \quad 5.50141700$

H $\quad 0.58801300-1.05104900 \quad 6.32019400$

H $\quad 1.88625000-2.24055000 \quad 6.09269700$

$\begin{array}{llll}\text { C } & -1.93479400 & 1.14976500 & 2.64889000\end{array}$

$\begin{array}{lllll}\text { C } & -1.78513700 & 1.89267100 & 3.97834600\end{array}$ $\begin{array}{lll}-2.88371100 & -0.04111400 & 2.74895400\end{array}$

$\begin{array}{lll}-2.35546800 & 1.84982000 & 1.91604200\end{array}$

$\begin{array}{lll}-1.14090600 & 2.77132600 & 3.86744700\end{array}$

$\begin{array}{lll}-2.76470500 & 2.23716400 & 4.32579400\end{array}$

$\begin{array}{lll}-1.35583900 & 1.23577500 & 4.73751300\end{array}$

$\begin{array}{lll}-2.95869500 & -0.56182800 & 1.78992800\end{array}$

$\begin{array}{lll}-2.54092600 & -0.75018900 & 3.50427000\end{array}$

$\begin{array}{lll}-3.88303000 & 0.30966800 & 3.02557000\end{array}$

$\begin{array}{lll}-0.16005100 & 1.59203600 & 1.61650300\end{array}$

$\begin{array}{llll}0.33879800 & 3.62126200 & -0.38035700\end{array}$

$\begin{array}{llll}1.36621000 & 4.41589200 & 0.43710600\end{array}$

$\begin{array}{lll}1.36800600 & 5.47641500 & 0.16124000\end{array}$

$\begin{array}{llll}1.13940400 & 4.34728200 & 1.50691500\end{array}$

$\begin{array}{lll}2.37379600 & 4.02019300 & 0.27325300\end{array}$

$\begin{array}{llll}-1.06893600 & 4.17168400 & -0.11183000\end{array}$

$\begin{array}{lll}-1.16067400 & 5.22118600 & -0.41406200\end{array}$

$-1.81033500 \quad 3.58642000-0.66469400$

$\begin{array}{lll}-1.30651700 & 4.10672300 & 0.95608100\end{array}$

$\begin{array}{llll}0.66567800 & 3.73997500 & -1.87479000\end{array}$

$\begin{array}{llll}1.66174800 & 3.33287600 & -2.07516000\end{array}$

$\begin{array}{llll}-0.06132800 & 3.17069500 & -2.46376000\end{array}$

$\begin{array}{llll}0.64285100 & 4.78090000 & -2.21661000\end{array}$

$\begin{array}{llll}0.38810800 & 2.26387600 & 0.00355000\end{array}$

$\begin{array}{lll}-0.56304700 & -1.95664700 & -0.28452600\end{array}$

$\begin{array}{llll}-0.80631600 & -3.33481900 & -0.16582900\end{array}$

$\begin{array}{llll}0.38274300 & -3.99026600 & 0.54834800\end{array}$

$\begin{array}{lll}-2.09558900 & -3.54836900 & 0.63654600\end{array}$

$\begin{array}{llll}-0.96116100 & -3.93499100 & -1.56960700\end{array}$

$\begin{array}{llll}1.29907800 & -3.83271600 & -0.02996500\end{array}$

$\begin{array}{llll}0.52364500 & -3.54205500 & 1.53612800\end{array}$

$\begin{array}{llll}0.23567300 & -5.06891000 & 0.67384400\end{array}$

$\begin{array}{lll}-2.93937600 & -3.07476400 & 0.12403500\end{array}$

$\begin{array}{lll}-2.32359100 & -4.61281200 & 0.76182900\end{array}$

$\begin{array}{lll}-2.00417100 & -3.09451000 & 1.62791600\end{array}$

$-1.14424600-5.01465900-1.52762300$

$\begin{array}{lll}-1.79814300 & -3.46413100 & -2.09414000\end{array}$

$\begin{array}{lll}-0.05227500 & -3.76246000 & -2.15481000\end{array}$

$\begin{array}{llll}2.16686600 & -0.47349100 & -3.34050900\end{array}$

$\begin{array}{llll}3.11010500 & 0.69815400 & -3.63714700\end{array}$

$\begin{array}{llll}2.97591400 & -1.73195500 & -3.00376900\end{array}$

$\begin{array}{llll}1.26015000 & -0.73866600 & -4.54808800\end{array}$

$\begin{array}{llll}2.53321400 & 1.59804100 & -3.87097800\end{array}$

$\begin{array}{llll}3.73670400 & 0.91058700 & -2.76506000\end{array}$

$\begin{array}{llll}3.76559100 & 0.47750300 & -4.48685000\end{array}$

$\begin{array}{llll}2.30284200 & -2.55773300 & -2.75335700\end{array}$

$\begin{array}{llll}3.60381300 & -2.04121200 & -3.84653400\end{array}$

$3.62734100-1.54827600-2.14300300$

$\begin{array}{lll}1.84477800 & -0.99216400 & -5.43934400\end{array}$

$\begin{array}{llll}0.57941500 & -1.56924000 & -4.33584700\end{array}$

$\begin{array}{llll}0.66064900 & 0.14940500 & -4.77144900\end{array}$

$\begin{array}{llll}-3.03179600 & 0.72681100 & -2.53433900\end{array}$

$\begin{array}{lll}-3.24699100 & -0.61722800 & -3.23851400\end{array}$

$\begin{array}{llll}-4.22080000 & 1.03874200 & -1.61788800\end{array}$

$\begin{array}{llll}-2.85476800 & 1.84308500 & -3.56992200\end{array}$

$\begin{array}{lll}-2.38715200 & -0.85389100 & -3.87306100\end{array}$

$\begin{array}{llll}-3.35827000 & -1.41685700 & -2.49978500\end{array}$

$\begin{array}{lll}-4.14456800 & -0.59991400 & -3.86651100\end{array}$

$\begin{array}{llll}-4.05672600 & 1.98456700 & -1.09180500\end{array}$

$\begin{array}{lll}-5.15310100 & 1.11990000 & -2.18747800\end{array}$

$\begin{array}{llll}-4.34281600 & 0.24689000 & -0.87185600\end{array}$

$\begin{array}{llll}-3.73884700 & 1.93545300 & -4.21022000\end{array}$

$\begin{array}{lll}-2.68951200 & 2.80338200 & -3.07174500\end{array}$

$\begin{array}{llll}-1.98931900 & 1.63394200 & -4.20650500\end{array}$ 


\begin{tabular}{|c|c|c|c|}
\hline \multicolumn{4}{|c|}{ dimer-C ( $\left.{ }^{t} \mathrm{BuOH}\right)$} \\
\hline \multicolumn{4}{|c|}{$H=-1935.263130 a u$} \\
\hline 0 & -0.72218500 & 0.86013500 & 2.70972000 \\
\hline $\mathrm{O}$ & -1.42961000 & 2.72129200 & 0.78421100 \\
\hline Th & -1.13808000 & 3.15712300 & 3.23661900 \\
\hline $\mathrm{C}$ & -1.91063800 & 3.70725100 & -0.16179700 \\
\hline $\mathrm{C}$ & -3.20805600 & 3.18586200 & -0.78477000 \\
\hline $\mathrm{C}$ & -2.18682200 & 5.02430300 & 0.56658800 \\
\hline $\mathrm{C}$ & -0.83479400 & 3.92718500 & -1.22750700 \\
\hline $\mathrm{H}$ & -3.02970200 & 2.25923200 & -1.34004200 \\
\hline $\mathrm{H}$ & -3.95210900 & 2.99581200 & -0.00506800 \\
\hline $\mathrm{H}$ & -3.62798800 & 3.91400300 & -1.48731100 \\
\hline $\mathrm{H}$ & -1.27470700 & 5.40265400 & 1.04024700 \\
\hline $\mathrm{H}$ & -2.53988500 & 5.78579300 & -0.13643700 \\
\hline $\mathrm{H}$ & -2.95919300 & 4.89060900 & 1.33131500 \\
\hline $\mathrm{H}$ & -1.15172000 & 4.68342000 & -1.95398300 \\
\hline $\mathrm{H}$ & 0.09348500 & 4.26942700 & -0.76049900 \\
\hline $\mathrm{H}$ & -0.63740900 & 2.99984600 & -1.77379900 \\
\hline $\mathrm{C}$ & -0.40733700 & -0.18448300 & 3.66007500 \\
\hline $\mathrm{C}$ & 0.92763200 & 0.14943200 & 4.32858800 \\
\hline $\mathrm{C}$ & -1.53797900 & -0.26199200 & 4.68891700 \\
\hline $\mathrm{C}$ & -0.29125600 & -1.52472800 & 5800 \\
\hline $\mathrm{H}$ & 1.72008600 & 0.20833400 & 3.57626300 \\
\hline $\mathrm{H}$ & 0.86630400 & 1.10615100 & 4.85568100 \\
\hline $\mathrm{H}$ & 1.20587500 & -0.61902400 & 5.05814200 \\
\hline $\mathrm{H}$ & -2.48807900 & -0.47595600 & 4.18973300 \\
\hline $\mathrm{H}$ & -1.35093700 & -1.05554800 & 5.42076100 \\
\hline $\mathrm{H}$ & -1.62788200 & 0.68035700 & 5.23801900 \\
\hline $\mathrm{H}$ & -0.04032200 & -2.32631600 & 3.63232200 \\
\hline $\mathrm{H}$ & -1.23765900 & -1.78749500 & 2.44675600 \\
\hline $\mathrm{H}$ & 0.50536300 & -1.48910400 & 2.17808800 \\
\hline Th & -0.94248000 & 0.44957000 & 0.24892000 \\
\hline $\mathrm{O}$ & -1.20163000 & 0.53551100 & -1.89692800 \\
\hline $\mathrm{O}$ & -2.58269700 & -0.92706100 & 17000 \\
\hline $\mathrm{O}$ & 1.15399400 & -0.08115000 & 28100 \\
\hline $\mathrm{O}$ & -3.21667400 & 3.66753200 & 3.50103100 \\
\hline 0 & 0.32156000 & 4.70368400 & 2.83884300 \\
\hline $\mathrm{O}$ & -0.69628700 & 3.06475400 & 5.35284700 \\
\hline$C$ & -3.64386500 & -1.85577500 & 0.48852700 \\
\hline $\mathrm{C}$ & -3.08148900 & -3.24202700 & 0.15145900 \\
\hline $\mathrm{C}$ & -4.34479800 & -1.88841000 & 1.85200700 \\
\hline $\mathrm{C}$ & -4.63339300 & -1.41055000 & -0.59397400 \\
\hline $\mathrm{H}$ & -2.57047200 & -3.21990800 & -0.81648600 \\
\hline $\mathrm{H}$ & -2.36010200 & -3.55780100 & 0.91227200 \\
\hline $\mathrm{H}$ & -3.87550100 & -3.99520900 & 0.10211100 \\
\hline $\mathrm{H}$ & -4.70520300 & -0.88874500 & 2.11460100 \\
\hline $\mathrm{H}$ & -5.20076200 & -2.57213700 & 1.84413600 \\
\hline $\mathrm{H}$ & -3.65373000 & -2.22009000 & 2.63340200 \\
\hline $\mathrm{H}$ & -5.48208100 & -2.09918500 & -0.67005700 \\
\hline $\mathrm{H}$ & -5.01979900 & -0.41204700 & -0.36556300 \\
\hline $\mathrm{H}$ & -4.13542400 & -1.36960400 & -1.56725100 \\
\hline$C$ & 2.52220300 & -0.34550300 & -0.08702500 \\
\hline $\mathrm{C}$ & 3.35248100 & 0.73229100 & 0.62078800 \\
\hline $\mathrm{C}$ & 2.86975900 & -1.73094000 & 0.47096700 \\
\hline $\mathrm{C}$ & 2.80178600 & -0.30855700 & -1.59318000 \\
\hline $\mathrm{H}$ & 3.09739000 & 1.72258700 & 0.22956300 \\
\hline $\mathrm{H}$ & 3.14865900 & 0.72674300 & 1.69598000 \\
\hline $\mathrm{H}$ & 4.42643800 & 0.57223900 & 0.47512900 \\
\hline $\mathrm{H}$ & 2.24165200 & -2.49650100 & 0.00364900 \\
\hline $\mathrm{H}$ & 3.91812000 & -1.98479400 & 0.27949400 \\
\hline $\mathrm{H}$ & 2.70717000 & -1.76570500 & 1.55287100 \\
\hline $\mathrm{H}$ & 3.85459200 & -0.52082900 & -1.80929200 \\
\hline
\end{tabular}

$2.18780700-1.05179900-2.11121200$

$\begin{array}{llll}2.56093900 & 0.67896200 & -1.99841800\end{array}$

$\begin{array}{llll}-1.29186800 & 0.26405200 & -3.28183400\end{array}$

$\begin{array}{llll}-2.64254700 & 0.76087800 & -3.81019100\end{array}$

$\begin{array}{lll}-0.15266300 & 0.98052000 & -4.01595500\end{array}$

$-1.17351900-1.25071500-3.48900200$

$\begin{array}{llll}-3.46490500 & 0.28607300 & -3.26639800\end{array}$

$\begin{array}{llll}-2.72879500 & 1.84462100 & -3.68476000\end{array}$

$\begin{array}{llll}-2.75809500 & 0.53323600 & -4.87553300\end{array}$

$\begin{array}{llll}0.81494800 & 0.64740200 & -3.63159000\end{array}$

$\begin{array}{lll}-0.18550500 & 0.77637800 & -5.09192000\end{array}$

$\begin{array}{llll}-0.22454200 & 2.06351600 & -3.87536100\end{array}$

$\begin{array}{lll}-1.23826300 & -1.51835300 & -4.54943000\end{array}$

$-0.21463800-1.61168900-3.10285700$

$\begin{array}{llll}-1.97602700 & -1.76907400 & -2.95483800\end{array}$

$\begin{array}{llll}-4.51328700 & 4.04550000 & 3.91135300\end{array}$

$\begin{array}{lll}-4.56121600 & 5.56967200 & 4.07110400\end{array}$

$\begin{array}{lll}-5.51860800 & 3.59195400 & 2.84645000\end{array}$

$\begin{array}{lll}-4.82681600 & 3.36581200 & 5.24838100\end{array}$

$\begin{array}{llll}-3.82428500 & 5.89513900 & 4.81251800\end{array}$

$\begin{array}{lll}-4.33031900 & 6.06215700 & 3.12100700\end{array}$

$\begin{array}{lll}-5.55029300 & 5.90762000 & 4.39904700\end{array}$

$\begin{array}{lll}-5.45494900 & 2.50837400 & 2.70451000\end{array}$

$\begin{array}{lll}-6.54547000 & 3.84170700 & 3.13513700\end{array}$

$\begin{array}{lll}-5.30790900 & 4.07640700 & 1.88785700\end{array}$

$\begin{array}{lll}-5.83154500 & 3.62178900 & 5.60200500\end{array}$

$\begin{array}{lll}-4.76485800 & 2.27776400 & 5.14452500\end{array}$

$\begin{array}{lll}-4.10432800 & 3.67818500 & 6.00821800\end{array}$

$\begin{array}{lll}1.29303300 & 5.72648600 & 2.79774000\end{array}$

$\begin{array}{lll}2.40896000 & 5.39245600 & 3.79378500\end{array}$

$\begin{array}{llll}1.87174000 & 5.80880500 & 1.38018500\end{array}$

$\begin{array}{lll}0.63242600 & 7.05824200 & 3.17425900\end{array}$

$\begin{array}{llll}2.00396300 & 5.33019700 & 4.80804900\end{array}$

$\begin{array}{lll}2.86238100 & 4.42728900 & 3.54563400\end{array}$

$\begin{array}{lll}3.19548400 & 6.15491500 & 3.78404400\end{array}$

$\begin{array}{lll}1.08975900 & 6.06458900 & 0.65838700\end{array}$

$\begin{array}{llll}2.65584400 & 6.57111500 & 1.31442600\end{array}$

$\begin{array}{llll}2.30432800 & 4.84547800 & 1.09206200\end{array}$

$\begin{array}{lll}1.35452000 & 7.88197700 & 3.15808500\end{array}$

$\begin{array}{lll}-0.17340500 & 7.29732500 & 2.47269400\end{array}$

$\begin{array}{lll}0.20356300 & 6.99863900 & 4.17970700\end{array}$

$\begin{array}{lll}-0.47790000 & 3.35804000 & 6.71909600\end{array}$

$\begin{array}{lll}-1.37934900 & 2.46731100 & 7.58192200\end{array}$

$\begin{array}{llll}0.99202400 & 3.09540000 & 7.06807400\end{array}$

$\begin{array}{lll}-0.81668700 & 4.83411000 & 6.95875500\end{array}$

$\begin{array}{lll}-2.43087500 & 2.61873500 & 7.32115200\end{array}$

$\begin{array}{lll}-1.13672500 & 1.41140100 & 7.42806600\end{array}$

$\begin{array}{lll}-1.25327300 & 2.69182200 & 8.64684000\end{array}$

$\begin{array}{llll}1.64979700 & 3.68901400 & 6.42680800\end{array}$

$\begin{array}{llll}1.20243100 & 3.35426700 & 8.11155700\end{array}$

$\begin{array}{lll}1.23925800 & 2.03879700 & 6.92682700\end{array}$

$\begin{array}{lll}-0.66584200 & 5.11732300 & 8.00628900\end{array}$

$\begin{array}{lll}-0.18281300 & 5.47311400 & 6.33565800\end{array}$

$\begin{array}{lll}-1.86185000 & 5.02898600 & 6.69734100\end{array}$

\section{Catc $\left({ }^{t} \mathrm{BuOH}\right)$}

$H=-967.61671 \mathrm{au}$

Th $\quad \begin{array}{llll}1.02950700 & 1.96780000 & -0.34433200\end{array}$

$\begin{array}{lllll}\text { C } & 3.60602100 & 0.66477600 & -3.53347200\end{array}$

$\begin{array}{lllll}\mathrm{H} & 3.83801600 & 1.73450800 & -3.55579700\end{array}$

H $\quad 4.36700700 \quad 0.13576300 \quad-4.11749000$

H $\quad 2.63588000 \quad 0.51456800 \quad-4.01848100$

C $\quad 3.19891800 \quad-1.32618200 \quad-2.04931100$ 


\begin{tabular}{|c|c|c|c|}
\hline 1 & 2.22477400 & -1.49187800 & -2.52080000 \\
\hline 1 & 3.94344200 & -1.93267200 & -2.57640600 \\
\hline & 3.14195200 & -1.67655800 & -1.01347600 \\
\hline 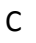 & 4.90603500 & 0.40244600 & -1.39513800 \\
\hline$y$ & 5.15031800 & 1.46968800 & -1.40079800 \\
\hline H & 4.86217800 & 0.06635000 & -0.35416300 \\
\hline 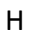 & 5.71607700 & -0.13774000 & -1.89712600 \\
\hline 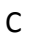 & -2.22167100 & 0.64054300 & -2.82362300 \\
\hline 1 & -1.40916300 & 0.03376300 & -3.23648600 \\
\hline 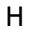 & -3.17289900 & 0.22365800 & -3.17185200 \\
\hline H & -2.12651100 & 1.65673200 & -3.21989200 \\
\hline - & -3.25384400 & 1.54090800 & -0.71296800 \\
\hline H & -4.24677000 & 1.16175100 & -0.97814800 \\
\hline I & -3.17945700 & 1.57456800 & 0.37881300 \\
\hline 1 & -3.16350100 & 2.56312500 & -1.09444800 \\
\hline 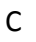 & -2.24837600 & -0.76578200 & -0.73721200 \\
\hline I & -2.16674900 & -0.75279100 & 0.35451200 \\
\hline $\mathrm{H}$ & -3.20281400 & -1.23189600 & -1.00489100 \\
\hline$y$ & -1.43936500 & -1.38766500 & -1.13433200 \\
\hline & 1.65963800 & 0.06901800 & 3.46851900 \\
\hline H & 0.78379100 & -0.49864000 & 3.13771400 \\
\hline $\mathrm{H}$ & 2.53745200 & -0.31463600 & 2.93848700 \\
\hline - & 1.80008500 & -0.10991000 & 4.54010500 \\
\hline [ & 0.22069400 & 2.09160900 & 3.88108500 \\
\hline$y$ & 0.07165400 & 3.15031600 & 3.64480100 \\
\hline$H$ & -0.66620000 & 1.53921900 & 3.55404700 \\
\hline - & 0.30606000 & 1.99168100 & 4.96859600 \\
\hline : & 2.70729300 & 2.35289500 & 3.59989800 \\
\hline 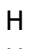 & 2.88686600 & 2.26217500 & 4.67667500 \\
\hline H & 3.59420000 & 1.98717500 & 3.07213600 \\
\hline $\mathrm{H}$ & 2.57923700 & 3.41374900 & 3.36137300 \\
\hline b & 2.56353600 & 0.88340300 & -1.39282100 \\
\hline $\mathrm{O}$ & 1.29474200 & 1.71985800 & 1.77724300 \\
\hline b & -0.89573200 & 1.19784200 & -0.91365200 \\
\hline C & -2.14256900 & 0.65896200 & -1.29293500 \\
\hline C & 3.55869500 & 0.16342200 & -2.08566700 \\
\hline C & 1.46879800 & 1.55991200 & 3.16751900 \\
\hline 0 & 1.14006800 & 4.05837500 & -0.84175800 \\
\hline C & 1.20974500 & 5.43032200 & -1.15997500 \\
\hline C & 0.39535200 & 6.21429300 & -0.12471800 \\
\hline$c$ & 2.67867500 & 5.86651500 & -1.12274400 \\
\hline 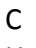 & 0.62780000 & 5.63745400 & -2.56289700 \\
\hline H & -0.64908800 & 5.88579300 & -0.13689900 \\
\hline $\mathrm{H}$ & 0.79683400 & 6.04359900 & 0.87945800 \\
\hline H & 0.41896500 & 7.29079600 & -0.32616100 \\
\hline $\mathrm{H}$ & 3.26355700 & 5.28607300 & -1.84359900 \\
\hline $\mathrm{H}$ & 2.78901000 & 6.92880500 & -1.36646200 \\
\hline 11 & 3.09817300 & 5.69768100 & -0.12554300 \\
\hline $\mathrm{H}$ & 0.65685100 & 6.69183500 & -2.85855100 \\
\hline & 1.19693800 & 5.05919300 & -3.29812900 \\
\hline & -0.41355400 & 5.30084300 & -2.59349100 \\
\hline
\end{tabular}

$\begin{array}{lll}1.56296800 & -2.24836000 & 2.97695000\end{array}$ $\begin{array}{llll}2.82540600 & 0.20297600 & 2.92594800\end{array}$ $\begin{array}{lll}2.94957600 & 1.21260200 & 2.52030100\end{array}$ $\begin{array}{llll}3.39793300 & 0.13529000 & 3.85777200\end{array}$ $\begin{array}{lll}3.25279500 & -0.50949000 & 2.21205400\end{array}$ $\begin{array}{lll}0.74383300 & 0.90929000 & 4.14111300\end{array}$ $\begin{array}{lll}-0.31821200 & 0.69307800 & 4.29913200\end{array}$ $\begin{array}{lll}1.25053800 & 0.86135400 & 5.11151900\end{array}$ $\begin{array}{lll}0.83610300 & 1.92642000 & 3.74858800\end{array}$ $\begin{array}{llll}0.20756100 & -0.28874800 & -0.18784200\end{array}$ $\begin{array}{lll}-1.90385100 & -0.64991500 & -0.61981500\end{array}$ $\begin{array}{llll}1.60780100 & 0.44345100 & -1.70623000\end{array}$ $\begin{array}{llll}-2.10774400 & 3.31701700 & 3.23678600\end{array}$ $\begin{array}{lll}-3.31431200 & 2.49376800 & 2.79781500\end{array}$ $\begin{array}{llll}-2.47260100 & 4.76051500 & 3.57951100\end{array}$ $\begin{array}{lll}-1.66326400 & 2.85471300 & 4.12650400\end{array}$ $\begin{array}{lll}-3.02506500 & 1.46605000 & 2.56041900\end{array}$ $\begin{array}{lll}-4.06089400 & 2.46239000 & 3.59721000\end{array}$ $\begin{array}{lll}-3.78509500 & 2.93522900 & 1.91245300\end{array}$ $\begin{array}{lll}-1.58700500 & 5.32357600 & 3.88496300\end{array}$ $\begin{array}{lll}-2.91924000 & 5.26262200 & 2.71511900\end{array}$ $\begin{array}{llll}-3.19701600 & 4.77890600 & 4.39904000\end{array}$ $\begin{array}{llll}-1.01744400 & 3.26725800 & -1.17366400\end{array}$ $\begin{array}{llll}-1.18363400 & 2.38750800 & -2.40300700\end{array}$ $\begin{array}{llll}0.06519600 & 4.32676200 & -1.35386700\end{array}$ $\begin{array}{llll}-1.97368200 & 3.76494500 & -0.96336300\end{array}$ $\begin{array}{lll}-1.94407300 & 1.62081900 & -2.23259300\end{array}$ $\begin{array}{lll}-1.48586200 & 2.99501800 & -3.26167500\end{array}$ $\begin{array}{llll}-0.23636100 & 1.89360700 & -2.64470000\end{array}$ $\begin{array}{llll}0.14681500 & 4.96567600 & -0.46896300\end{array}$ $\begin{array}{llll}1.03455400 & 3.85242000 & -1.52915700\end{array}$ $\begin{array}{llll}-0.16973600 & 4.96322800 & -2.21333500\end{array}$ $\begin{array}{lll}0.82216800 & -2.34328400 & -0.29727400\end{array}$ $\begin{array}{llll}1.18561900 & -3.70153400 & -0.36994300\end{array}$ $\begin{array}{llll}1.14161800 & -4.14666000 & -1.83671000\end{array}$ $\begin{array}{llll}2.60485100 & -3.86989700 & 0.18649500\end{array}$ $\begin{array}{llll}0.19598000 & -4.52708400 & 0.46112100\end{array}$ $\begin{array}{llll}0.13617600 & -4.00458600 & -2.24544700\end{array}$ $\begin{array}{llll} & 1.84057000 & -3.55170700 & -2.43314700\end{array}$ $\begin{array}{llll}1.41130300 & -5.20325000 & -1.94422700\end{array}$ $\begin{array}{llll}2.64517600 & -3.54806700 & 1.23183100\end{array}$ $\begin{array}{llll}2.93261900 & -4.91423400 & 0.13732100\end{array}$ $\begin{array}{llll}3.31198400 & -3.26064800 & -0.38483200\end{array}$ $\begin{array}{llll}0.44771700 & -5.59336700 & 0.44158800\end{array}$ $\begin{array}{llll}0.20334100 & -4.19039800 & 1.50258100\end{array}$ $\begin{array}{lll}-0.81924400 & -4.40781200 & 0.06973100\end{array}$ $\begin{array}{llll}2.64423400 & 0.48386200 & -2.65893600\end{array}$ $2.15542200-0.16599600-3.96014000$ $\begin{array}{llll}3.03091800 & 1.94533300 & -2.91945600\end{array}$ $\begin{array}{lll}3.85666600 & -0.28065900 & -2.11200800\end{array}$ $\begin{array}{llll}1.86531000 & -1.20569500 & -3.77843100\end{array}$ $\begin{array}{llll}1.28165000 & 0.36916200 & -4.34677900\end{array}$ $\begin{array}{llll}2.93157700 & -0.15577500 & -4.73362100\end{array}$ $\begin{array}{llll}3.33815400 & 2.42618700 & -1.98500000\end{array}$ $\begin{array}{llll}3.85983200 & 2.01960400 & -3.63230700\end{array}$ $\begin{array}{llll}2.18104300 & 2.50001500 & -3.33022300\end{array}$ $\begin{array}{lll}4.68912300 & -0.28408200 & -2.82460700\end{array}$ $\begin{array}{llll}4.20457700 & 0.17901800 & -1.18083500\end{array}$ $\begin{array}{llll}3.58125500 & -1.31797200 & -1.89870300\end{array}$ $\begin{array}{llll}-3.16635700 & -1.25608500 & -0.74458900\end{array}$ $\begin{array}{llll}-3.11994900 & -2.27724400 & -1.88855100\end{array}$ $\begin{array}{llll}-3.51612400 & -1.95928000 & 0.57401300\end{array}$ $-4.21175400-0.17571100-1.05050000$ 


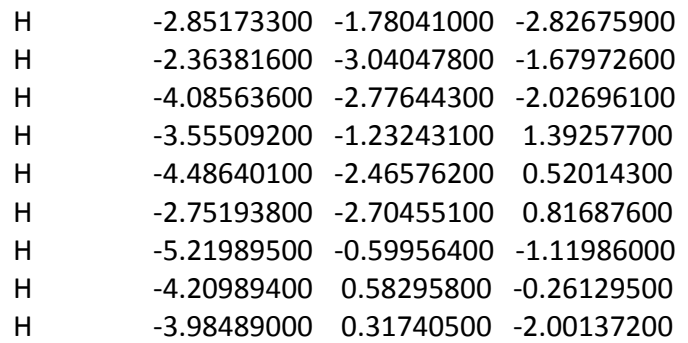

\section{$\mathrm{TS}_{1 \mathrm{C}-2 \mathrm{C}}\left({ }^{\mathrm{t}} \mathrm{BuOH}\right)$}

$H=-1351.920751 \mathrm{au}$

N $\quad-0.84533900 \quad 2.56928700 \quad 0.56815100$

$\begin{array}{llll}\text { C } & -1.17435800 & 2.60912700 & 1.81692200\end{array}$

$\begin{array}{lllll}\mathrm{N} & -1.78972100 & 3.19626200 & 2.70263300\end{array}$

$\begin{array}{lllll}\mathrm{O} & -0.25936900 & 1.01886000 & 2.37906700\end{array}$

$\begin{array}{lllll}\text { C } & 0.89073400 & 1.07686700 & 3.24008900\end{array}$

$\begin{array}{lllll}\text { C } & 0.64708700 & 0.11403300 & 4.40453200\end{array}$

$\begin{array}{llll}\mathrm{H} & 1.50397700 & 0.08909000 & 5.08676800\end{array}$

$\begin{array}{llll}\mathrm{H} & -0.23478400 & 0.41953300 & 4.97589000\end{array}$

$\begin{array}{llll}\text { H } & 0.47365200 & -0.89797800 & 4.02714500\end{array}$

$\begin{array}{llll}\text { C } & 2.12172800 & 0.62516800 & 2.43670800\end{array}$

$\begin{array}{llll}\mathrm{H} & 2.30291100 & 1.30037400 & 1.58900000\end{array}$

$\begin{array}{llll}H & 3.02883600 & 0.63903100 & 3.04937000\end{array}$

$\begin{array}{llll}\text { H } & 2.00343200 & -0.40469700 & 2.07437300\end{array}$

$\begin{array}{llll}\text { C } & 1.14172000 & 2.49500600 & 3.75852600\end{array}$

$\begin{array}{llll}\mathrm{H} & 0.31169700 & 2.85290200 & 4.37026700\end{array}$

$\begin{array}{llll}\mathrm{H} & 2.04693500 & 2.50812000 & 4.37474900\end{array}$

$\begin{array}{llll}H & 1.28362800 & 3.19528800 & 2.93005900\end{array}$

Th $\quad-0.20865600 \quad 0.08429000 \quad 0.18597000$

O $\quad-2.14674300-0.32958000-0.64582400$

$\begin{array}{lllll}0 & & 1.25743900 & 0.42164600 & -1.35547500\end{array}$

$\begin{array}{llll}\text { C } & -2.42692800 & 2.75602400 & 3.92208000\end{array}$

C $\quad-3.81452200 \quad 2.18215000 \quad 3.62234800$

C $\quad-2.50513800 \quad 3.92189900 \quad 4.90570600$

H $\quad-1.81287100 \quad 1.95489200 \quad 4.35757400$

H $\quad-3.74357100 \quad 1.32733200 \quad 2.94364000$

H $\quad-4.29896600 \quad 1.84786700 \quad 4.54600800$

$\begin{array}{llll}\mathrm{H} & -4.45001800 & 2.94124500 & 3.15488200\end{array}$

H $\quad-1.51090200 \quad 4.32430100 \quad 5.12048700$

$\begin{array}{llll}H & -3.11232000 & 4.73198700 & 4.48877000\end{array}$

$\begin{array}{lllll}\mathrm{H} & -2.95915000 & 3.60017300 & 5.84822800\end{array}$

C $\quad-1.50341100 \quad 3.58474900 \quad-0.28576800$

C $\quad-1.41169600 \quad 3.13884700 \quad-1.73973700$

$\begin{array}{lllll}\text { C } & -0.85719800 & 4.95333300 & -0.07288800\end{array}$

H $\quad-2.56337400 \quad 3.65309200 \quad 0.00120600$

H $\quad-1.90523700 \quad 2.17290000 \quad-1.88616100$

$\mathrm{H} \quad-1.89316600 \quad 3.86936500 \quad-2.39767200$

H $\quad-0.36440600 \quad 3.04204800 \quad-2.04567500$

$\begin{array}{llll}\mathrm{H} & -0.93497800 & 5.26004400 & 0.97393800\end{array}$

$\begin{array}{lllll}\mathrm{H} & 0.20212600 & 4.92097300 & -0.34921900\end{array}$

H $\quad-1.35083200 \quad 5.71297700 \quad-0.68885900$

$\begin{array}{lllll}0 & 0.32773500 & -1.91791900 & 0.79009000\end{array}$

C $\quad 0.58283600 \quad-3.28468000 \quad 1.02816700$

C $\quad 0.39782000-4.05247700 \quad-0.28610900$

C $\quad 2.02291300 \quad-3.44275100 \quad 1.52979400$

$\begin{array}{llll}\text { C } & -0.40419500 & -3.79744200 & 2.08308600\end{array}$

$\mathrm{H} \quad-0.62206000-3.91890200 \quad-0.66072000$

H $\quad 1.09233000 \quad-3.67702700 \quad-1.04446400$

H $\quad 0.57816000 \quad-5.12510500-0.15498200$

H $\quad 2.16085200 \quad-2.90244900 \quad 2.47216900$

$\begin{array}{llll}\text { H } & 2.27288000 & -4.49529200 & 1.70246500\end{array}$ $\begin{array}{lll}2.72561000 & -3.03819900 & 0.79441800\end{array}$

$\begin{array}{lll}-0.25086400 & -4.86207500 & 2.29127200\end{array}$

$\begin{array}{lll}-0.28192800 & -3.24422000 & 3.01977200\end{array}$

$\begin{array}{lll}-1.43330800 & -3.65849500 & 1.73767600\end{array}$

$\begin{array}{lll}2.24713500 & 0.50496800 & -2.35611500\end{array}$

$\begin{array}{llll}1.56756900 & 0.40646900 & -3.72677700\end{array}$

$\begin{array}{llll}2.97446800 & 1.84767700 & -2.21927100\end{array}$

$3.23666300-0.65115300-2.17171200$

$\begin{array}{llll}1.02983000 & -0.54308900 & -3.81428800\end{array}$

$\begin{array}{llll}0.84562200 & 1.21965600 & -3.85155500\end{array}$

$\begin{array}{lll}2.29736900 & 0.46543900 & -4.54170300\end{array}$

$\begin{array}{llll}3.44528900 & 1.92557100 & -1.23382400\end{array}$

$\begin{array}{llll}3.75344400 & 1.96108800 & -2.98119900\end{array}$

$\begin{array}{lll}2.26708600 & 2.67612400 & -2.32498600\end{array}$

$4.02623300-0.62687000-2.93073600$

$3.70680400-0.59355900-1.18460100$

$\begin{array}{llll}2.71618800 & -1.61106000 & -2.24686100\end{array}$

$\begin{array}{lll}-3.35493600 & -0.85459900 & -1.15279200\end{array}$

$\begin{array}{lll}-3.10722300 & -1.38244100 & -2.57034800\end{array}$

$\begin{array}{lll}-3.81904900 & -1.99212600 & -0.23590500\end{array}$

$\begin{array}{lll}-4.40636200 & 0.26046400 & -1.17691900\end{array}$

$\begin{array}{lll}-2.74744400 & -0.57610400 & -3.21735900\end{array}$

$\begin{array}{lll}-2.34797000 & -2.17116900 & -2.55359200\end{array}$

$\begin{array}{lll}-4.02155600 & -1.79516700 & -3.01065200\end{array}$

$\begin{array}{lll}-3.97645900 & -1.61807000 & 0.78112700\end{array}$

$\begin{array}{lll}-4.75708000 & -2.43466100 & -0.58845700\end{array}$

$\begin{array}{lll}-3.06017900 & -2.77974700 & -0.19719000\end{array}$

$\begin{array}{lll}-5.37078200 & -0.11065800 & -1.54062500\end{array}$

$\begin{array}{lll}-4.55105200 & 0.66627700 & -0.17082300\end{array}$

$-4.08611000 \quad 1.07658300-1.83187200$

\section{Int-2C ( ${ }^{\mathrm{BuOH}}$ )}

$H=-1351.932062 \mathrm{au}$

$\begin{array}{llll}\mathrm{N} & -0.41168200 & 2.05311200 & 0.27243100\end{array}$

$\begin{array}{llll}\text { C } & -0.72976300 & 1.88558900 & 1.57456800\end{array}$

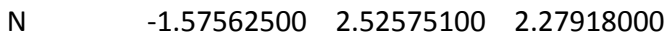

$\begin{array}{llll}\text { O } & -0.01589200 & 0.71390200 & 2.04648000\end{array}$

$\begin{array}{llll}\text { C } & 1.28823300 & 0.90518200 & 2.72074400\end{array}$

$\begin{array}{lllll}\text { C } & 1.30869900 & -0.13283000 & 3.83592100\end{array}$

$\begin{array}{llll}\mathrm{H} & 2.27348000 & -0.11958500 & 4.35306500\end{array}$

$\begin{array}{llll}\mathrm{H} & 0.52189300 & 0.07011700 & 4.56727300\end{array}$

H $\quad \begin{array}{llll}\text { H } & 1.14931800 & -1.13279300 & 3.42463600\end{array}$

$\begin{array}{llll}\text { C } & 2.39186000 & 0.62444300 & 1.69797700\end{array}$

$\begin{array}{llll}\mathrm{H} & 2.34317400 & 1.34179600 & 0.86906700\end{array}$

$\begin{array}{lllll}H & 3.38090900 & 0.73259500 & 2.15321500\end{array}$

$\begin{array}{llll}\mathrm{H} & 2.33391700 & -0.40991700 & 1.33224500\end{array}$

$\begin{array}{llll}\text { C } & 1.44444200 & 2.31848000 & 3.26962200\end{array}$

$\begin{array}{llll}\mathrm{H} & 0.67741600 & 2.55339200 & 4.00875700\end{array}$

$\begin{array}{llll}\mathrm{H} & 2.42119100 & 2.39482500 & 3.75790800\end{array}$

$\begin{array}{llll}\mathrm{H} & 1.40401000 & 3.06480500 & 2.47205300\end{array}$

Th $\quad 0.12903200-0.23237700 \quad-0.47750800$

O $\quad-1.69641000 \quad-0.74957200-1.44729700$

$\begin{array}{lllll}0 & 1.58791100 & 0.03482900 & -2.01902100\end{array}$

$\begin{array}{llll}\text { C } & -1.97886300 & 2.12611200 & 3.61126800\end{array}$

C $\quad-3.31723900 \quad 1.38223500 \quad 3.53128400$

$\begin{array}{llll}\text { C } & -2.10701900 & 3.37140900 & 4.49130100\end{array}$

$\begin{array}{llll}\text { H } & -1.25155700 & 1.44046500 & 4.07509500\end{array}$

H $\quad-3.21582000 \quad 0.46302900 \quad 2.94572100$

$\begin{array}{llll}\mathrm{H} & -3.67972300 & 1.11394300 & 4.53018500\end{array}$

$\begin{array}{llll}H & -4.06937300 & 2.01247300 & 3.04552400\end{array}$

H $\quad-1.15544800 \quad 3.90832700 \quad 4.56115900$

$\begin{array}{llll}H & -2.84138300 & 4.05936900 & 4.05958500\end{array}$

$\begin{array}{llll}\mathrm{H} & -2.42916200 & 3.10870000 & 5.50490800\end{array}$ 
$\begin{array}{lll}-1.04135400 & 3.18523300 & -0.40828000\end{array}$

$\begin{array}{lll}-0.96338200 & 2.97173300 & -1.91795000\end{array}$

$\begin{array}{lll}-0.38351500 & 4.50580300 & -0.00051800\end{array}$

$\begin{array}{llll}-2.09857700 & 3.23317700 & -0.10668900\end{array}$

$\begin{array}{lll}-1.49100500 & 2.05888700 & -2.21884700\end{array}$

$\begin{array}{llll}-1.42292100 & 3.80731600 & -2.45567100\end{array}$

$\begin{array}{llll}0.07997200 & 2.89674000 & -2.24815800\end{array}$

$\begin{array}{lll}-0.46754200 & 4.64730500 & 1.08000200\end{array}$

$0.67643200 \quad 4.50838400-0.28041400$

$\begin{array}{lll}-0.87023500 & 5.35472500 & -0.49407400\end{array}$

$\begin{array}{llll}0.68510100 & -2.13516200 & 0.39033200\end{array}$

$\begin{array}{lll}0.88278800 & -3.46464600 & 0.82305800\end{array}$

$0.71132600-4.39834200-0.38124600$

$\begin{array}{llll}2.30108500 & -3.60018100 & 1.38914500\end{array}$

$\begin{array}{lll}-0.15569200 & -3.79984900 & 1.89971800\end{array}$

$\begin{array}{lll}-0.29328100 & -4.29066500 & -0.80261600\end{array}$

$1.43883500-4.14869200-1.16013200$

$\begin{array}{llll}0.85443400 & -5.44743100 & -0.09995400\end{array}$

$\begin{array}{llll}2.43746600 & -2.93946000 & 2.25145300\end{array}$

$\begin{array}{llll}2.50488000 & -4.62641500 & 1.71363300\end{array}$

$\begin{array}{llll}3.03918800 & -3.32835100 & 0.62771200\end{array}$

$\begin{array}{lll}-0.04102500 & -4.82764200 & 2.26116900\end{array}$

$\begin{array}{lll}-0.05503700 & -3.12402400 & 2.75469700\end{array}$

$\begin{array}{lll}-1.16768800 & -3.68896500 & 1.49734100\end{array}$

$\begin{array}{llll}2.52600000 & 0.04013900 & -3.07560100\end{array}$

$\begin{array}{llll}1.77068500 & 0.21334700 & -4.39745400\end{array}$

$\begin{array}{llll}3.49451800 & 1.20767800 & -2.85913200\end{array}$

$\begin{array}{llll}3.28534500 & -1.29073700 & -3.06614300\end{array}$

$\begin{array}{lll}1.06528300 & -0.61118600 & -4.54178200\end{array}$

$\begin{array}{llll}1.20529400 & 1.15038400 & -4.39136500\end{array}$

$\begin{array}{llll}2.45806300 & 0.23090300 & -5.25010500\end{array}$

$\begin{array}{llll}4.02348700 & 1.09166000 & -1.90755800\end{array}$

$\begin{array}{llll}4.23937400 & 1.26147100 & -3.66040400\end{array}$

$\begin{array}{llll}2.94689900 & 2.15495300 & -2.83382800\end{array}$

$\begin{array}{llll}4.03484200 & -1.32852000 & -3.86407800\end{array}$

$\begin{array}{llll}3.79586400 & -1.42952200 & -2.10770100\end{array}$

$\begin{array}{llll}2.59104900 & -2.12518700 & -3.20778200\end{array}$

$\begin{array}{lll}-2.86342200 & -1.28558300 & -2.04044600\end{array}$

$\begin{array}{lll}-2.45351700 & -2.12101900 & -3.25708700\end{array}$

$\begin{array}{llll}-3.57563200 & -2.16142400 & -1.00425300\end{array}$

$-3.77033700-0.12798500-2.46881700$

$\begin{array}{lll}-1.92800400 & -1.49561700 & -3.98566900\end{array}$

$\begin{array}{llll}-1.78301300 & -2.93130800 & -2.95312300\end{array}$

$-3.32641800-2.56302300-3.74958400$

$\begin{array}{lll}-3.84630900 & -1.56626500 & -0.12600600\end{array}$

$\begin{array}{lll}-4.49060200 & -2.60199400 & -1.41458200\end{array}$

$-2.91917100-2.97505800-0.67923700$

$\begin{array}{lll}-4.69927000 & -0.49737500 & -2.91651500\end{array}$

$\begin{array}{lll}-4.02675500 & 0.49287600 & -1.60491800\end{array}$

$\begin{array}{llll}-3.26254100 & 0.50123700 & -3.20642000\end{array}$

\section{Int-3C (' $\mathrm{BuOH})$}

$H=-1585.403873$ au

$\begin{array}{llll}\mathrm{N} & -0.56107600 & 0.76929300 & 1.84012200\end{array}$

C $\quad 0.23014200 \quad-0.04440900 \quad 2.58685400$

N $\quad 0.00580400 \quad-0.50541300 \quad 3.75285100$

$\begin{array}{lllll}0 & 1.35596100 & -0.41059000 & 1.74847400\end{array}$

$\begin{array}{llll}\text { C } & 2.71104800 & 0.11971400 & 2.01376900\end{array}$

$\begin{array}{llll}\text { C } & 3.57825400 & -0.98657200 & 2.61296400\end{array}$

$\begin{array}{llll}\mathrm{H} & 4.62945400 & -0.68092100 & 2.59063600\end{array}$

H $\quad 3.31813800 \quad-1.20055400 \quad 3.65037700$

$\begin{array}{llll}\text { H } & 3.47762100 & -1.90534700 & 2.02811600\end{array}$

$\begin{array}{llll}\text { C } & 3.26493500 & 0.50451600 & 0.64432700\end{array}$ $\begin{array}{llll}2.68436500 & 1.31983700 & 0.20323000\end{array}$ $\begin{array}{llll}4.30046100 & 0.84621500 & 0.73889400\end{array}$ $\begin{array}{llll}3.25813300 & -0.35033300 & -0.03865400\end{array}$ $\begin{array}{llll}2.65763700 & 1.34395800 & 2.92171500\end{array}$ $\begin{array}{lll}2.20632900 & 1.11042300 & 3.88783300\end{array}$ $\begin{array}{lll}3.67574700 & 1.70656300 & 3.09421000\end{array}$ $\begin{array}{llll}2.08318600 & 2.14966800 & 2.45843000\end{array}$ $\begin{array}{lll}-0.10710600 & -0.09327900 & -0.54606100\end{array}$ $\begin{array}{llll}-1.89392900 & 0.53299500 & -1.55135700\end{array}$ $\begin{array}{llll}1.33871800 & -0.20615700 & -2.14831200\end{array}$ $\begin{array}{llll}0.70635900 & -1.60841800 & 4.37446300\end{array}$ $\begin{array}{lll}-0.30717400 & -2.72531400 & 4.65364700\end{array}$ $\begin{array}{llll}1.35323500 & -1.15788600 & 5.68776600\end{array}$ $\begin{array}{lll}1.47973400 & -2.02384600 & 3.71411400\end{array}$ $\begin{array}{lll}-0.77798700 & -3.06371800 & 3.72618700\end{array}$ $\begin{array}{llll}0.17858600 & -3.58639600 & 5.12674000\end{array}$ $\begin{array}{lll}-1.09673500 & -2.36289300 & 5.32040100\end{array}$ $\begin{array}{lll}2.12551900 & -0.39911800 & 5.52624000\end{array}$ $\begin{array}{llll}0.59304200 & -0.72147700 & 6.34414500\end{array}$ $\begin{array}{lll}1.81486900 & -2.00386200 & 6.20898300\end{array}$ $\begin{array}{lll}-1.87595700 & 1.16804600 & 2.37286400\end{array}$ $\begin{array}{lll}-1.76235600 & 2.05835100 & 3.61504000\end{array}$ $\begin{array}{lll}-2.83166300 & -0.00647400 & 2.60947800\end{array}$ $\begin{array}{lll}-2.31823400 & 1.78000600 & 1.57286700\end{array}$ $\begin{array}{lll}-1.11701200 & 2.92235600 & 3.41852500\end{array}$ $\begin{array}{lll}-2.74857700 & 2.43330400 & 3.91080700\end{array}$ $\begin{array}{lll}-1.33516900 & 1.49233200 & 4.44562900\end{array}$ $\begin{array}{lll}-2.91940300 & -0.62439300 & 1.70897200\end{array}$ $\begin{array}{lll}-2.47445500 & -0.63529600 & 3.42695000\end{array}$ $\begin{array}{lll}-3.83174600 & 0.36409000 & 2.86090400\end{array}$ $\begin{array}{lll}0.11825800 & 2.18541800 & 0.93591800\end{array}$ $\begin{array}{llll}0.31624700 & 3.82032300 & -0.31464100\end{array}$ $\begin{array}{lll}1.27863300 & 4.62348300 & 0.56102500\end{array}$ $\begin{array}{llll}1.22780800 & 5.68944000 & 0.31684500\end{array}$ $\begin{array}{lll}1.02791000 & 4.51119600 & 1.62136500\end{array}$ $\begin{array}{llll}2.30796100 & 4.28493400 & 0.41104200\end{array}$ $\begin{array}{llll}-1.13011100 & 4.25576900 & -0.08221500\end{array}$ $\begin{array}{llll}-1.27255500 & 5.29898800 & -0.38175000\end{array}$ $\begin{array}{llll}-1.81222200 & 3.62993500 & -0.66416000\end{array}$ $\begin{array}{lll}-1.40109800 & 4.17125300 & 0.97573600\end{array}$ $\begin{array}{llll}0.69301100 & 3.93630300 & -1.78720200\end{array}$ $\begin{array}{llll}1.71162000 & 3.57380300 & -1.95206200\end{array}$ $0.01045400 \quad 3.34771300-2.40707300$ $\begin{array}{llll}0.64080900 & 4.97934000 & -2.11329300\end{array}$ $\begin{array}{llll}0.45168900 & 2.42108200 & 0.02098500\end{array}$ $\begin{array}{llll}-0.56665700 & -2.16873100 & -0.33762000\end{array}$ $\begin{array}{lll}-0.83628700 & -3.55349500 & -0.30608000\end{array}$ $\begin{array}{lll}0.28642200 & -4.25017000 & 0.47031600\end{array}$ $\begin{array}{llll}-2.18368200 & -3.77528200 & 0.38897100\end{array}$ $\begin{array}{llll}-0.88896700 & -4.07872000 & -1.74485100\end{array}$ $\begin{array}{llll}1.24882800 & -4.07673500 & -0.02208400\end{array}$ $\begin{array}{llll}0.34635500 & -3.85190500 & 1.48740000\end{array}$ $\begin{array}{llll}0.12002400 & -5.33136900 & 0.52993900\end{array}$ $\begin{array}{llll}-2.98012500 & -3.25858900 & -0.15620000\end{array}$ $\begin{array}{llll}-2.43672700 & -4.83995400 & 0.43980900\end{array}$ $\begin{array}{lll}-2.15485500 & -3.37790600 & 1.40805800\end{array}$ $\begin{array}{llll}-1.09926500 & -5.15361100 & -1.76988100\end{array}$ $\begin{array}{llll}-1.67079000 & -3.56139800 & -2.30932100\end{array}$ $\begin{array}{llll}0.06790800 & -3.90435600 & -2.24726100\end{array}$ $\begin{array}{llll}2.17006800 & -0.42297200 & -3.26622400\end{array}$ $\begin{array}{llll}3.08839300 & 0.79090400 & -3.44959200\end{array}$ $3.00615300-1.68629100-3.02763400$ $\begin{array}{lll}1.28785100 & -0.60584800 & -4.50700000\end{array}$ 


\begin{abstract}
$\begin{array}{llll}2.49195200 & 1.69369000 & -3.61397200\end{array}$
$\begin{array}{llll}3.70213700 & 0.94264300 & -2.55595400\end{array}$

$\begin{array}{llll}3.75757200 & 0.66104600 & -4.30736100\end{array}$

$\begin{array}{llll}2.35029300 & -2.54623300 & -2.86008400\end{array}$

$\begin{array}{llll}3.65235900 & -1.90730700 & -3.88429300\end{array}$

$\begin{array}{llll}3.64251200 & -1.56497000 & -2.14483900\end{array}$

$\begin{array}{llll}1.88995800 & -0.76972900 & -5.40766600\end{array}$

$\begin{array}{llll}0.62416200 & -1.46631100 & -4.37535100\end{array}$

$\begin{array}{llll}0.66948500 & 0.28362300 & -4.66426300\end{array}$

$\begin{array}{llll}-3.06396900 & 0.66448800 & -2.33109500\end{array}$

$-3.32326300-0.65460900-3.06715700$

$\begin{array}{lll}-4.23895200 & 0.99115400 & -1.40305500\end{array}$

$\begin{array}{llll}-2.85723800 & 1.79651900 & -3.34379600\end{array}$

$\begin{array}{lll}-2.47831300 & -0.89746100 & -3.71943100\end{array}$

$\begin{array}{lll}-3.44824200 & -1.47030300 & -2.34848700\end{array}$

$\begin{array}{lll}-4.22742700 & -0.59702800 & -3.68309400\end{array}$

$\begin{array}{llll}-4.04761300 & 1.92156000 & -0.85893500\end{array}$

$\begin{array}{lll}-5.17141000 & 1.10871400 & -1.96603100\end{array}$

$\begin{array}{lll}-4.37826800 & 0.19039100 & -0.67026400\end{array}$

$\begin{array}{llll}-3.73810600 & 1.92584900 & -3.98206700\end{array}$

$\begin{array}{lll}-2.66964100 & 2.74329000 & -2.82743400\end{array}$

$\begin{array}{llll}-1.99733400 & 1.57803100 & -3.98485800\end{array}$
\end{abstract}

\section{$\mathrm{TS}_{3 \mathrm{C}-4 \mathrm{C}}\left({ }^{\mathrm{t}} \mathrm{BuOH}\right)$}

\section{$H=-1585.405774 a u$}

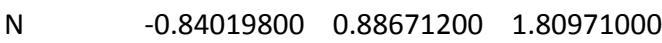

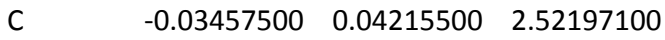

$\begin{array}{llll}\mathrm{N} & -0.27526400 & -0.45581800 & 3.66584600\end{array}$

$\begin{array}{lllll}\mathrm{O} & 1.07196700 & -0.29949000 & 1.66868400\end{array}$

$\begin{array}{llll}\text { C } & 2.44594600 & 0.18928300 & 1.93955700\end{array}$

$\begin{array}{llll}C & 3.28467000 & -0.94769400 & 2.51957000\end{array}$

$\begin{array}{llll}\mathrm{H} & 4.34299300 & -0.66813100 & 2.49435800\end{array}$

H $\quad 3.02641300 \quad-1.16792300 \quad 3.55612500$

H $\quad 3.15863500 \quad-1.85595300 \quad 1.92332800$

$\begin{array}{llll}\text { C } & 3.00157300 & 0.57974200 & 0.57256500\end{array}$

$\begin{array}{lllll}\mathrm{H} & 2.42258800 & 1.40092000 & 0.14162100\end{array}$

H $\quad 4.04038800 \quad 0.90977300 \quad 0.67103000$

$\begin{array}{llll}\mathrm{H} & 2.99000300 & -0.27092300 & -0.11666500\end{array}$

$\begin{array}{lllll}\text { C } & 2.41565400 & 1.39942100 & 2.86524400\end{array}$

H $\quad 1.96690900 \quad 1.15718600 \quad 3.83083500$

$\begin{array}{llll}\mathrm{H} & 3.43975900 & 1.74378000 & 3.03926800\end{array}$

H $\quad \begin{array}{llll}\text { H } & 1.85139800 & 2.21871300 & 2.41434200\end{array}$

Th $\quad-0.35952600 \quad-0.07637100-0.67283300$

$\begin{array}{lllll}0 & -2.15859600 & 0.48974100 & -1.67917200\end{array}$

$\begin{array}{lllll}0 & 1.06287900 & -0.21384500 & -2.27906300\end{array}$

C $\quad 0.41329700 \quad-1.58195300 \quad 4.26109800$

$\begin{array}{llll}\text { C } & -0.61589100 & -2.68763200 & 4.52377300\end{array}$

$\begin{array}{lllll}\text { C } & 1.07184800 & -1.16360100 & 5.57899200\end{array}$

$\mathrm{H} \quad 1.17755600-1.99346400 \quad 3.58819800$

$\begin{array}{llll}\mathrm{H} & -1.09628800 & -3.00187200 & 3.59291200\end{array}$

H $\quad-0.13937800 \quad-3.56419900 \quad 4.97681200$

$\mathrm{H} \quad-1.39577800 \quad-2.32708300 \quad 5.20263800$

$\begin{array}{llll}\mathrm{H} & 1.84979900 & -0.40833500 & 5.42904900\end{array}$

$\begin{array}{lllll}H & 0.31977000 & -0.73541900 & 6.24980000\end{array}$

$\begin{array}{llll}\mathrm{H} & 1.52897700 & -2.02506600 & 6.07794700\end{array}$

$\begin{array}{llll}\text { C } & -2.16402800 & 1.25629300 & 2.34153000\end{array}$

$\begin{array}{llll}\text { C } & -2.07423900 & 2.09883100 & 3.61751500\end{array}$

$\begin{array}{llll}\text { C } & -3.10557800 & 0.06174400 & 2.51334900\end{array}$

$\begin{array}{llll}\mathrm{H} & -2.59440000 & 1.89327900 & 1.55594600\end{array}$

$\begin{array}{llll}H & -1.43606400 & 2.97538200 & 3.46095800\end{array}$

H $\quad-3.06864700 \quad 2.45236700 \quad 3.91160900$

$\begin{array}{llll}\mathrm{H} & -1.65455800 & 1.50650100 & 4.43330600\end{array}$

H $\quad-3.16593500 \quad-0.52390500 \quad 1.58931900$

\section{Int-4C (' $\left.{ }^{\mathrm{BuOH}}\right)$}

$H=-1585.414477 a u$

$\begin{array}{llll}\mathrm{N} & -0.63513500 & 0.83781800 & 2.07858700\end{array}$

$\begin{array}{llll}\text { C } & 0.24335500 & -0.04971600 & 2.69545300\end{array}$

N $\quad-0.02651800 \quad-0.64926800 \quad 3.77369000$

$\begin{array}{lll}-2.76062300 & -0.59203200 & 3.31637600\end{array}$ $\begin{array}{lll}-4.11540700 & 0.41103400 & 2.75454000\end{array}$ $\begin{array}{lll}1.87467500 & 1.02872200\end{array}$ $0.05134400 \quad 3.66774300-0.29753900$ $\begin{array}{lll}1.03367500 & 4.44623900 & 0.58219000\end{array}$ $\begin{array}{lll}01815700 & 5.51412600 & 0.33951900\end{array}$ $\begin{array}{lll}0.77409200 & 4.33886100 & 1.64073000\end{array}$ $\begin{array}{lll}2.05387200 & 4.07793600 & 0.43721900\end{array}$ $\begin{array}{lll}4.07673700 & 4.17219900 & -0.07310200\end{array}$ $-2.08436900-3.58106400-0.66183100$ $\begin{array}{lll}-1.65640800 & 4.09511000 & 0.98269400\end{array}$ $0.43638500 \quad 3.80011000 \quad-1.77088700$ $1.44309400 \quad 3.40577500-1.93864300$ $\begin{array}{lll}-0.26209300 & 3.23828900 & -2.39913800\end{array}$ $\begin{array}{llll}0.41824100 & 4.84726700 & -2.09006800\end{array}$ $\begin{array}{llll}0.12974200 & 2.27859600 & 0.03322300\end{array}$ $\begin{array}{lll}-0.79223000 & -2.15986700 & -0.40569000\end{array}$ $-1.07411000-3.54136400-0.36143700$ $0.04021800 \quad-4.24334300 \quad 0.42248500$ $\begin{array}{lll}-2.42567000 & -3.74669000 & 0.33089600\end{array}$ $1.12824800-4.07797500-1.79626700$ $1.00562600-4.07957800-0.06741800$ $\begin{array}{lll}0.09961400 & -3.84152500 & 1.43831000\end{array}$ $\begin{array}{lll}-2.68742200 & -4.80876700 & 0.39146000\end{array}$ $\begin{array}{lll}-2.39800900 & -3.33968000 & 1.34629900\end{array}$ $-1.34882400-5.15095800-1.81414400$ $-1.90349500-3.55698800-2.36653900$ $\begin{array}{llll}-0.16851000 & -3.91631900 & -2.29749000\end{array}$ $1.87873700-0.44310800-3.40737300$ $2.78416600 \quad 0.77526800-3.61971000$ $2.72656400-1.69610600-3.15877700$ $0.97948000-0.65056900-4.63134100$ $\begin{array}{llll}2.17852900 & 1.67160400 & -3.78492700\end{array}$ $3.41065800 \quad 0.94237500-2.73768800$ $3.44056500 \quad 0.63914300-4.48624500$ $2.07962100-2.55890800-2.97238300$ $3.36608400-1.92393900-4.01856800$ $3.37014300-1.55600400-2.28400900$ $1.57022800-0.82481000-5.53744100$ $0.32334600-1.51305200-4.47730100$ $\begin{array}{llll}0.35394600 & 0.23282300 & -4.79388200\end{array}$ $\begin{array}{llll}-3.33051900 & 0.63641900 & -2.45395200\end{array}$ $\begin{array}{lll}-3.58892800 & -0.66832700 & -3.21511500\end{array}$ $\begin{array}{llll}-4.50342300 & 0.94267000 & -1.51662200\end{array}$ $\begin{array}{lll}-3.12520200 & 1.78970500 & -3.44228800\end{array}$ $\begin{array}{llll}-2.74341400 & -0.89871400 & -3.87105000\end{array}$ $-3.71462300-1.49761700-2.51219200$ $-4.49262500-0.59890900-3.83048100$ $\begin{array}{llll}-4.31130600 & 1.86158000 & -0.95372300\end{array}$ $\begin{array}{llll}-5.43696000 & 1.07145500 & -2.07524800\end{array}$ $\begin{array}{lll}-4.64126900 & 0.12594500 & -0.80125000\end{array}$ $\begin{array}{llll}-4.00833800 & 1.93479400 & -4.07400000\end{array}$ $\begin{array}{lll}-2.93223900 & 2.72396600 & -2.90574900\end{array}$ $\begin{array}{llll}-2.26841600 & 1.58329100 & -4.09146900\end{array}$ 
$\begin{array}{llll}\text { O } & 1.32620500 & -0.24520600 & 1.82405900\end{array}$

$\begin{array}{lllll}\text { C } & 2.68725200 & 0.28306400 & 2.11725900\end{array}$

$\begin{array}{llll}\text { C } & 3.55123900 & -0.83384200 & 2.69403600\end{array}$

H $\quad 4.59885600 \quad-0.51603300 \quad 2.69279000$

$\begin{array}{llll}H & 3.28272600 & -1.08114100 & 3.72193800\end{array}$

H $\quad 3.46861500-1.73420800 \quad 2.07900300$

$\begin{array}{lllll}\text { C } & 3.22421800 & 0.69984900 & 0.75237700\end{array}$

$\begin{array}{llll}\mathrm{H} & 2.59472900 & 1.48123700 & 0.31547900\end{array}$

$\begin{array}{lllll}\mathrm{H} & 4.24078600 & 1.09176500 & 0.85440300\end{array}$

$\begin{array}{llll}H & 3.26720500 & -0.15537600 & 0.06998100\end{array}$

$\begin{array}{llll}\text { C } & 2.60159400 & 1.48166300 & 3.05391200\end{array}$

$\begin{array}{llll}\mathrm{H} & 2.13713200 & 1.21849700 & 4.00787900\end{array}$

H $\quad 3.61146500 \quad 1.84838800 \quad 3.26055500$

$\begin{array}{llll}\mathrm{H} & 2.03626100 & 2.29704800 & 2.59530700\end{array}$

Th $\quad-0.07218400 \quad 0.10057500 \quad-0.67592400$

$\begin{array}{lllll}0 & -1.88845500 & 0.56554800 & -1.71017900\end{array}$

$\begin{array}{lllll}0 & 1.35646700 & -0.16724700 & -2.25832700\end{array}$

C $\quad 0.69558300 \quad-1.78291200 \quad 4.31846100$

$\begin{array}{llll}\text { C } & -0.30252000 & -2.92411200 & 4.53674000\end{array}$

C $\quad 1.33635300 \quad-1.38975700 \quad 5.65245600$

$\begin{array}{llll}\mathrm{H} & 1.47338600 & -2.13779600 & 3.62899200\end{array}$

H $\quad-0.77559100 \quad-3.21867100 \quad 3.59657600$

$\begin{array}{llll}\mathrm{H} & 0.20281400 & -3.80140800 & 4.95445300\end{array}$

$\begin{array}{llll}\mathrm{H} & -1.08905700 & -2.61305800 & 5.23188300\end{array}$

H $\quad 2.06817300 \quad-0.58428500 \quad 5.53699100$

$\begin{array}{llll}\mathrm{H} & 0.56611900 & -1.04069400 & 6.34785400\end{array}$

$\begin{array}{llll}\mathrm{H} & 1.84460600 & -2.24827000 & 6.10364700\end{array}$

$\begin{array}{lllll}\text { C } & -1.93569100 & 1.21870100 & 2.66725700\end{array}$

C $\quad-1.79018700 \quad 1.96465300 \quad 3.99525600$

$\begin{array}{lllll}\text { C } & -2.88958000 & 0.03108600 & 2.76483800\end{array}$

$\begin{array}{llll}\mathrm{H} & -2.34870700 & 1.91711100 & 1.92882000\end{array}$

H $\quad-1.14237400 \quad 2.84080500 \quad 3.88523100$

$\begin{array}{llll}H & -2.77016000 & 2.31346000 & 4.33708100\end{array}$

H $\quad \begin{array}{llll}H & -1.36675100 & 1.30813900 & 4.75810300\end{array}$

H $\quad-2.95961300 \quad-0.49282200 \quad 1.80683100$

$\begin{array}{llll}H & -2.55531800 & -0.67626100 & 3.52555100\end{array}$

$\begin{array}{lllll}H & -3.88980500 & 0.38631500 & 3.03206500\end{array}$

H $\quad-0.15910700 \quad 1.63292100 \quad 1.62341600$

$\begin{array}{llll}\text { C } & 0.33671800 & 3.64226500 & -0.42790100\end{array}$

$\begin{array}{lllll}\text { C } & 1.36786200 & 4.39605000 & 0.42314200\end{array}$

$\begin{array}{llll}\mathrm{H} & 1.38342100 & 5.46520800 & 0.18336100\end{array}$

$\begin{array}{llll}\mathrm{H} & 1.13279100 & 4.29568200 & 1.48885500\end{array}$

$\begin{array}{lllll}\text { H } & 2.37240300 & 3.99551100 & 0.25242600\end{array}$

$\begin{array}{lllll}\text { C } & -1.06519500 & 4.20199100 & -0.14905600\end{array}$

H $\quad-1.14229400 \quad 5.26086600 \quad-0.42102800$

H $\quad-1.81172300 \quad 3.64226400 \quad-0.72073300$

H $\quad-1.30924500 \quad 4.10992600 \quad 0.91548500$

C $\quad 0.67676800 \quad 3.80915700 \quad-1.91487200$

H $\quad \begin{array}{lllll}\mathrm{C} & 1.66870800 & 3.39536500 & -2.12148000\end{array}$

H $\quad-0.05175900 \quad 3.27036100 \quad-2.52957100$

H $\quad 0.67097400 \quad 4.86186200 \quad-2.21933400$

$\begin{array}{lllll}\text { O } & 0.36446300 & 2.27095300 & -0.09841300\end{array}$

$\begin{array}{lllll}0 & -0.51081400 & -1.99449800 & -0.28794400\end{array}$

$\begin{array}{lllll}\text { C } & -0.78472800 & -3.37204900 & -0.22093400\end{array}$

$\begin{array}{llll}\text { C } & 0.36058000 & -4.06937000 & 0.52392200\end{array}$

C $\quad-2.10988200 \quad-3.58179200 \quad 0.52173200$

$\begin{array}{llll}\text { C } & -0.89155600 & -3.92807400 & -1.64669500\end{array}$

$\mathrm{H} \quad \begin{array}{llll}\mathrm{H} & 1.30452900 & -3.90883600 & -0.00727600\end{array}$

$\begin{array}{llll}H & 0.46301000 & -3.65535900 & 1.53143200\end{array}$

$\begin{array}{llll}H & 0.19172500 & -5.14886300 & 0.60805200\end{array}$

H $\quad-2.92297900 \quad-3.07773400 \quad-0.01083300$

H $\quad-2.36244000 \quad-4.64457900 \quad 0.60822500$

$\begin{array}{lllll}\mathrm{H} & -2.05071300 & -3.15726100 & 1.52877000\end{array}$
H $\quad-1.10624500-5.00271700-1.64544400$

H $\quad-1.69027100-3.41722400-2.19309900$

H $\quad 0.04736200 \quad-3.76566000 \quad-2.18571200$

C $\quad 2.16229600-0.47586000 \quad-3.37202700$

$\begin{array}{lllll}\text { C } & 3.05855600 & 0.72891000 & -3.68158700\end{array}$

C $\quad 3.02157400 \quad-1.70111600 \quad-3.03703800$

C $\quad 1.25687900 \quad-0.78034100-4.57125600$

H $\quad 2.44569600 \quad 1.60624300 \quad-3.90957400$

$\begin{array}{lllll}\mathrm{H} & 3.68648700 & 0.96612200 & -2.81670600\end{array}$

H $\quad 3.71274300 \quad 0.53209700 \quad-4.53824600$

H $\quad 2.38257100 \quad-2.55037100 \quad-2.77603400$

H $\quad 3.65415600 \quad-1.99125500 \quad-3.88318200$

H $\quad 3.67283000-1.48787600 \quad-2.18274900$

H $\quad 1.84290200-1.02017300 \quad-5.46545700$

H $\quad 0.60586600 \quad-1.63156000 \quad-4.34790200$

H $\quad 0.62560800 \quad 0.08514500 \quad-4.79605300$

$\begin{array}{lllll}\text { C } & -3.05672900 & 0.68823200 & -2.48937800\end{array}$

C $\quad-3.31832000-0.63899200 \quad-3.21004100$

C $\quad-4.23071400 \quad 1.02481300 \quad-1.56237900$

C $\quad-2.85332400 \quad 1.81125100 \quad-3.51292900$

$\mathrm{H} \quad-2.46959000-0.89377700 \quad-3.85256300$

H $\quad-3.45048100 \quad-1.44458100 \quad-2.48118700$

H $\quad-4.21837800 \quad-0.58611800-3.83264900$

H $\quad-4.03457600 \quad 1.95882000 \quad-1.02603100$

H $\quad \begin{array}{llll}-5.16392200 & 1.14109600 & -2.12450000\end{array}$

$\begin{array}{llll}\mathrm{H} & -4.37196000 & 0.22810400 & -0.82491700\end{array}$

H $\quad-3.73747600 \quad 1.93821500 \quad-4.14735900$

$\begin{array}{llll}\mathrm{H} & -2.65711400 & 2.76023500 & -3.00431600\end{array}$

$\mathrm{H} \quad-1.99770400 \quad 1.58470000 \quad-4.15691600$

\section{$\mathrm{HN}\left(\mathrm{SiMe}_{3}\right)_{2}$}

$H=-302.410499 a u$

Si $\quad \begin{array}{llll}0.45649500 & 3.94977700 & 1.87415400\end{array}$

Si $\quad \begin{array}{llll}2.18174700 & 1.56278700 & 3.22696800\end{array}$

$\begin{array}{llll}\mathrm{N} & 1.07030400 & 2.30994100 & 2.08071600\end{array}$

$\begin{array}{lllll}\text { C } & -0.23814600 & 4.06178900 & 0.11522300\end{array}$

H $\quad-0.65993600 \quad 5.05512500 \quad-0.07129400$

$\begin{array}{llll}\mathrm{H} & 0.54177200 & 3.88270800 & -0.63189100\end{array}$

$\begin{array}{llll}\mathrm{H} & -1.03808200 & 3.33088800 & -0.04711000\end{array}$

$\begin{array}{llll}\text { C } & -0.92339200 & 4.34640100 & 3.11065900\end{array}$

$\begin{array}{llll}\text { H } & -0.56015200 & 4.28534900 & 4.14152900\end{array}$

$\begin{array}{llll}\mathrm{H} & -1.31611000 & 5.35732600 & 2.95333300\end{array}$

$\begin{array}{llll}H & -1.75414200 & 3.64074600 & 3.00700900\end{array}$

$\begin{array}{llll}\text { C } & 1.85207900 & 5.20980500 & 2.09673300\end{array}$

$\begin{array}{llll}\mathrm{H} & 2.64754900 & 5.04459700 & 1.36342200\end{array}$

$\begin{array}{llll}\mathrm{H} & 1.47530800 & 6.23021800 & 1.96604000\end{array}$

$\begin{array}{llll}\mathrm{H} & 2.29670100 & 5.15133800 & 3.09573100\end{array}$

C $\quad \begin{array}{llll}1.82068900 & -0.29679900 & 3.22257700\end{array}$

H $\quad 0.79450200 \quad-0.50107000 \quad 3.54422900$

H $\quad \begin{array}{llll}H & 1.95462000 & -0.72766600 & 2.22402300\end{array}$

H $\quad 2.49870000 \quad-0.82618400 \quad 3.90045900$

$\begin{array}{llll}\text { C } & 1.90316900 & 2.27476400 & 4.95836900\end{array}$

$\begin{array}{llll}\mathrm{H} & 2.08271100 & 3.35448500 & 4.98904300\end{array}$

$\begin{array}{llll}\mathrm{H} & 0.87876800 & 2.09234500 & 5.29747400\end{array}$

H $\quad 2.58670300 \quad 1.81035300 \quad 5.67762800$

$\begin{array}{lllll}\text { C } & 3.99266800 & 1.83737300 & 2.73884800\end{array}$

H $\quad 4.67179500 \quad 1.35168600 \quad 3.44872100$

$\begin{array}{llll}\mathrm{H} & 4.19622500 & 1.42774300 & 1.74387500\end{array}$

$\begin{array}{llll}\mathrm{H} & 4.23777600 & 2.90416400 & 2.71603900\end{array}$

$\begin{array}{llll}\text { H } & 0.72860600 & 1.66566400 & 1.37754900\end{array}$

EtOH 
$\mathrm{H}=-154.88845 \mathrm{au}$

C $\quad 3.13311100 \quad 1.42451700 \quad-0.28394200$

C $\quad 3.92112000 \quad 2.43419700 \quad-1.09406200$

$\mathrm{H} \quad 4.43962800 \quad 1.93793600 \quad-1.92100500$

H $\quad 3.25094500 \quad 3.19034000 \quad-1.51603800$

$\begin{array}{lllll}\mathrm{H} & 4.66506000 & 2.93738900 & -0.46678600\end{array}$

$\begin{array}{lllll}O & 2.19156500 & 0.81043900 & -1.14817300\end{array}$

$\begin{array}{lllll}\mathrm{H} & 1.68856000 & 0.16698400 & -0.63063100\end{array}$

$\begin{array}{llll}\mathrm{H} & 3.81864300 & 0.67754200 & 0.15192800\end{array}$

$\begin{array}{llll}\mathrm{H} & 2.62919000 & 1.93098100 & 0.55716300\end{array}$

\section{${ }^{t} \mathrm{BuOH}$}

$H=-233.438507 a u$

C $\quad 3.14643200 \quad 1.42482900 \quad-0.24479100$

$\begin{array}{lllll}\text { C } & 4.08387800 & 0.34876600 & 0.31269000\end{array}$

$\begin{array}{llll}\mathrm{H} & 4.84617900 & 0.78044200 & 0.97186200\end{array}$

$\begin{array}{llll}H & 3.52242800 & -0.39270700 & 0.89687500\end{array}$

H $\quad 4.58805800 \quad-0.17450500 \quad-0.50671000$

$\begin{array}{llll}\text { C } & 2.39341700 & 2.12997000 & 0.88793200\end{array}$

$\begin{array}{llll}\mathrm{H} & 3.08082700 & 2.64486800 & 1.56937200\end{array}$

$\begin{array}{llll}\mathrm{H} & 1.69435300 & 2.86601800 & 0.47692000\end{array}$

H $\quad 1.81779900 \quad 1.40750000 \quad 1.48213900$

C $\quad 3.92145300 \quad 2.43289900 \quad-1.08895000$

H $\quad 4.42578600 \quad 1.92438800 \quad-1.91735300$

H $\quad 3.23832700 \quad 3.17705900 \quad-1.51188400$

H $\quad 4.67450200 \quad 2.95164200 \quad-0.48613800$

$\begin{array}{lllll}\mathrm{O} & 2.21128700 & 0.82903100 & -1.14860500\end{array}$

$\begin{array}{lllll}\mathrm{H} & 1.69839200 & 0.17983900 & -0.64464900\end{array}$

\section{${ }^{i} \mathrm{Pr}-\mathrm{N}=\mathrm{C}=\mathrm{N}-{ }^{-} \mathrm{Pr}$}

$H=-384.317346$ au

C $\quad 1.12914400 \quad 1.19179700 \quad-0.46540600$

$\mathrm{N} \quad 2.23801700 \quad 1.09261300 \quad-0.97495800$

$\begin{array}{llll}\mathrm{N} & 0.00570900 & 1.44919400 & -0.04777500\end{array}$

$\begin{array}{lllll}\text { C } & 3.48828900 & 0.66075800 & -0.35561100\end{array}$

C $\quad 4.07962100 \quad-0.47484800 \quad-1.18991600$

$\begin{array}{llll}\text { C } & 3.34336600 & 0.27959600 & 1.11571900\end{array}$

H $\quad 4.16881000 \quad 1.52070700 \quad-0.42783700$

$\begin{array}{lllll}\mathrm{H} & 4.16643400 & -0.17685700 & -2.23808400\end{array}$

H $\quad 5.07356400 \quad-0.74630400 \quad-0.82008500$

H $\quad 3.43905800 \quad-1.36177000 \quad-1.13975100$

$\begin{array}{llll}\mathrm{H} & 2.92379800 & 1.10528800 & 1.69869900\end{array}$

$\begin{array}{llll}\mathrm{H} & 2.68388900 & -0.58871200 & 1.22689000\end{array}$

$\begin{array}{llll}H & 4.31728300 & 0.02099200 & 1.54330900\end{array}$

C $\quad-1.14029500 \quad 0.53821600 \quad-0.00861200$

C $\quad-2.23379300 \quad 1.08681700 \quad-0.92321400$

$\begin{array}{lllll}C & & -1.62035900 & 0.41117500 & 1.43545100\end{array}$

H $\quad-0.84513900-0.45736200-0.37185600$

H $\quad-1.87987300 \quad 1.15900100 \quad-1.95552900$

H $\quad \begin{array}{lllll}H & -3.11291000 & 0.43428300 & -0.90337800\end{array}$

H $\quad-2.53518100 \quad 2.08683300 \quad-0.59580000$

$\begin{array}{llll}H & -0.83260000 & 0.00431000 & 2.07614800\end{array}$

H $\quad-1.90418900 \quad 1.39280200 \quad 1.82788500$

H $\quad-2.48979900 \quad-0.25173200 \quad 1.49410300$

\section{isourea (EtOH)}

$H=-539.240420 \mathrm{au}$

C $\quad \begin{array}{llll}1.24808900 & 1.07189500 & -0.63951100\end{array}$

N $\quad 2.42254800 \quad 1.51264200 \quad-0.84125800$

N $\quad \begin{array}{llll}N & 0.16935400 & 1.57929400 & -1.35985200\end{array}$

C $\quad 3.60863300 \quad 0.90149300 \quad-0.24784100$

$\begin{array}{llll}\text { C } & 3.78613600 & -0.58084700 & -0.59541300\end{array}$ $\begin{array}{lll}3.68637600 & 1.14945000 & 1.26254700\end{array}$

$\begin{array}{lll}4.45427400 & 1.43503000 & -0.70328900\end{array}$

$\begin{array}{lll}3.68880800 & -0.73698800 & -1.67469500\end{array}$

$\begin{array}{llll}4.78145800 & -0.92549000 & -0.29337900\end{array}$

$\begin{array}{llll}3.04519300 & -1.20129000 & -0.08426800\end{array}$

$\begin{array}{lll}3.57167400 & 2.21604800 & 1.47977100\end{array}$

$\begin{array}{lll}2.89732700 & 0.60397400 & 1.78755800\end{array}$

$\begin{array}{lll}4.65577500 & 0.82450300 & 1.65698500\end{array}$

$\begin{array}{lll}-1.01297300 & 2.08087200 & -0.64351000\end{array}$

$\begin{array}{lll}-2.15707200 & 2.25293700 & -1.63877300\end{array}$

$\begin{array}{lll}-0.72278400 & 3.37393200 & 0.12392400\end{array}$

$\begin{array}{lll}-1.29197300 & 1.30890400 & 0.08203700\end{array}$

$\begin{array}{llll}-2.36399800 & 1.31542300 & -2.16270800\end{array}$

$\begin{array}{llll}-3.06971400 & 2.57933600 & -1.13070700\end{array}$

$\begin{array}{llll}-1.90776000 & 3.01163400 & -2.39040200\end{array}$

$\begin{array}{llll}0.10390500 & 3.23533300 & 0.82678800\end{array}$

$\begin{array}{lll}-0.44780800 & 4.18091700 & -0.56551300\end{array}$

$\begin{array}{lll}-1.60200000 & 3.70066600 & 0.68969200\end{array}$

$\begin{array}{llll}0.52858200 & 2.25535600 & -2.02568700\end{array}$

$\begin{array}{lll}0.91752500 & 0.09955000 & 0.27040200\end{array}$

$0.14097400-1.01195700-0.20334300$

$\begin{array}{lll}-0.23902200 & -1.85277900 & 0.99643400\end{array}$

$\begin{array}{llll}0.65180900 & -2.20423000 & 1.52463000\end{array}$

$-0.84659400-1.27495200 \quad 1.69914700$

$\begin{array}{lll}-0.81667500 & -2.72543300 & 0.67620200\end{array}$

$-0.74545200-0.65784500-0.73945300$

$\begin{array}{llll}0.74797200 & -1.59006900 & -0.91297200\end{array}$

\section{isourea ( $\left.{ }^{t} \mathrm{BuOH}\right)$}

$H=-617.784508 \mathrm{au}$

C $\quad \begin{array}{llll}1.03619800 & 0.94641700 & -0.65416600\end{array}$

$\begin{array}{llll}\mathrm{N} & 2.20630600 & 1.42193400 & -0.80543600\end{array}$

N $\quad \begin{array}{llll}\text { N } & -0.02295800 & 1.47342200 & -1.41658200\end{array}$

$\begin{array}{llll}\text { C } & 3.39825000 & 0.90753500 & -0.13688300\end{array}$

C $\quad 3.75857300 \quad-0.53158800-0.51667800$

$\begin{array}{llll}\text { C } & 3.34248400 & 1.10002000 & 1.38248000\end{array}$

H $\quad 4.21584200 \quad 1.54072400 \quad-0.50837100$

$\begin{array}{llll}\mathrm{H} & 3.75978300 & -0.65650000 & -1.60427600\end{array}$

H $\quad 4.75965900-0.77979300 \quad-0.14725700$

H $\quad 3.05395300 \quad-1.24532800 \quad-0.08330600$

$\begin{array}{llll}\mathrm{H} & 3.11685400 & 2.14283100 & 1.62720300\end{array}$

$\begin{array}{llll}\mathrm{H} & 2.57084100 & 0.46688000 & 1.82764800\end{array}$

$\begin{array}{llll}\mathrm{H} & 4.30776700 & 0.84721300 & 1.83529800\end{array}$

C $\quad \begin{array}{llll}\text { C } & -1.02407800 & 2.28927500 & -0.69429400\end{array}$

C $\quad-2.20543300 \quad 2.55231300 \quad-1.62302400$

C $\quad-0.43206200 \quad 3.59359700 \quad-0.15341000$

$\begin{array}{llll}\mathrm{H} & -1.36449700 & 1.68739400 & 0.15368700\end{array}$

H $\quad-2.64258800 \quad 1.61742000-1.98519700$

H $\quad-2.98408000 \quad 3.12568000 \quad-1.11020100$

$\begin{array}{lllll}\mathrm{H} & -1.88755700 & 3.13354400 & -2.49711800\end{array}$

$\begin{array}{llll}\mathrm{H} & 0.43889700 & 3.40038300 & 0.47864800\end{array}$

H $\quad-0.10986700 \quad 4.24535300 \quad-0.97441400$

$\begin{array}{llll}\mathrm{H} & -1.17362200 & 4.14114500 & 0.43825000\end{array}$

H $\quad 0.41205900 \quad 2.04405400 \quad-2.13711700$

$\begin{array}{lllll}0 & 0.69828600 & -0.02444200 & 0.24442000\end{array}$

C $\quad-0.06732700-1.22050400 \quad-0.12862000$

$\begin{array}{lllll}\text { C } & 0.33511500 & -2.23167300 & 0.94413400\end{array}$

H $\quad 1.40556100-2.44857200 \quad 0.89792600$

$\begin{array}{llll}\mathrm{H} & 0.10753100 & -1.84043900 & 1.94010600\end{array}$

$\begin{array}{lllll}H & -0.21306800 & -3.16886100 & 0.80713200\end{array}$

C $\quad-1.57026700-0.95819600-0.04922100$

H $\quad-1.83671800 \quad-0.52791500 \quad 0.92109600$

H $\quad-1.89517900 \quad-0.28623100 \quad-0.84496800$ 


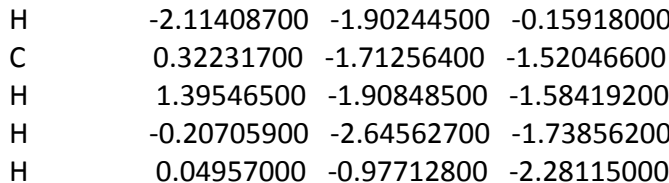

\section{ad-A ('BuOH)}

$H=-1175.904537 \mathrm{au}$

$\begin{array}{llll}\text { U } & 0.13564600 & 1.22449000 & 0.02814600\end{array}$

Si $\quad \begin{array}{llll}0.73961700 & 3.47611500 & 2.13931100\end{array}$

Si $\quad \begin{array}{llll}1.63620300 & 0.79468300 & 3.46377500\end{array}$

Si $\quad-3.22202100 \quad 1.03283300 \quad 1.52007900$

Si $\quad \begin{array}{lllll}-2.94969300 & 0.26645100 & -1.38840000\end{array}$

Si $\quad \begin{array}{llll}1.17647200 & -2.18473000 & -0.79873300\end{array}$

Si $\quad 3.30444000 \quad-0.00420900 \quad-0.97598900$

$\begin{array}{lllll}\mathrm{N} & 0.85576100 & 1.68663800 & 2.14790200\end{array}$

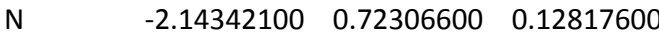

N $\quad \begin{array}{llll}\text { N } & 1.62877700 & -0.47388600 & -0.64656900\end{array}$

$\begin{array}{lllll}\text { C } & 3.84583800 & -0.37257200 & -2.75983500\end{array}$

H $\quad 3.12497900 \quad-0.00000900 \quad-3.49287100$

$\begin{array}{lllll}\mathrm{H} & 4.81705700 & 0.09117900 & -2.96806200\end{array}$

H $\quad 3.95851800 \quad-1.44969400 \quad-2.92230100$

$\begin{array}{llll}\text { C } & 4.60273300 & -0.81247000 & 0.15370900\end{array}$

H $\quad 4.87721900-1.81455700 \quad-0.18541700$

H $\quad \begin{array}{llll}5.51374500 & -0.20254300 & 0.15074200\end{array}$

H $\quad 4.25720000 \quad-0.89266700 \quad 1.18761700$

$\begin{array}{llll}\text { C } & 3.43950600 & 1.86977800 & -0.67403000\end{array}$

H $\quad 2.78610000 \quad 2.45982500 \quad-1.32415000$

$\begin{array}{llll}\mathrm{H} & 3.22023500 & 2.12143800 & 0.37015300\end{array}$

$\begin{array}{llll}\mathrm{H} & 4.46777300 & 2.19467500 & -0.86893100\end{array}$

$\begin{array}{lllll}\text { C } & -1.65505000 & 0.23194300 & -2.77831700\end{array}$

H $\quad-0.77700500 \quad-0.38560100-2.55983000$

H $\quad-2.12148200 \quad-0.19896400 \quad-3.67172700$

H $\quad-1.30911100 \quad 1.23453000 \quad-3.03385100$

C $\quad-4.25901800 \quad 1.53460300 \quad-1.92564000$

H $\quad-4.65401700 \quad 1.27011700 \quad-2.91337500$

H $\quad-5.10633400 \quad 1.57910700 \quad-1.23536000$

$\mathrm{H} \quad-3.82724000 \quad 2.53852000 \quad-1.99157200$

C $\quad-3.75688500-1.45230800 \quad-1.40608000$

$\mathrm{H} \quad-4.59807300-1.53681100 \quad-0.71583000$

H $\quad-4.13532400-1.64601500-2.41695400$

H $\quad-3.03918900 \quad-2.24115400 \quad-1.16457200$

$\begin{array}{lllll}\text { C } & -2.35882000 & 0.59102800 & 3.14224500\end{array}$

H $\quad-2.17551400 \quad-0.48745600 \quad 3.19487000$

H $\quad-1.40184600 \quad 1.10747200 \quad 3.24298400$

$\begin{array}{llll}\mathrm{H} & -2.99495800 & 0.86531300 & 3.99126200\end{array}$

$\begin{array}{llll}\text { C } & -4.82032400 & 0.00065200 & 1.51773600\end{array}$

$\begin{array}{llll}\text { H } & -5.38202900 & 0.26463900 & 2.42196200\end{array}$

$\begin{array}{llll}\mathrm{H} & -5.47670800 & 0.18792000 & 0.66329900\end{array}$

H $\quad-4.60983800 \quad-1.07198600 \quad 1.56401600$

$\begin{array}{llll}\text { C } & -3.75544000 & 2.85547100 & 1.55687700\end{array}$

$\begin{array}{llll}\mathrm{H} & -4.34611100 & 3.06938600 & 2.45498100\end{array}$

H $\quad-2.89627600 \quad 3.53253900 \quad 1.55273000$

$\begin{array}{llll}\text { H } & -4.37215300 & 3.09812200 & 0.68570300\end{array}$

$\begin{array}{llll}\text { C } & 0.18587200 & 3.75957900 & 0.35695400\end{array}$

H $\quad 0.85240400 \quad 4.52507500 \quad-0.06412800$

H $\quad 0.34945400 \quad 3.40058300 \quad-1.52413100$

$\begin{array}{llll}\mathrm{H} & -0.83894800 & 4.15700800 & 0.31894200\end{array}$

$\begin{array}{lllll}\text { C } & -0.47887000 & 4.14962000 & 3.43093300\end{array}$

$\begin{array}{llll}\text { H } & -0.10857700 & 4.00488700 & 4.45042200\end{array}$

$\begin{array}{llll}\mathrm{H} & -0.61491800 & 5.22599900 & 3.27456300\end{array}$

$\begin{array}{llll}H & -1.45999900 & 3.67290400 & 3.36184800\end{array}$ $\begin{array}{lll}2.40007100 & 4.34117100 & 2.46366200\end{array}$ $\begin{array}{llll}3.18657000 & 4.00660700 & 1.78109000\end{array}$ $\begin{array}{lll}2.27051100 & 5.42093200 & 2.32457600\end{array}$ $\begin{array}{lll}2.74896100 & 4.18207600 & 3.48886000\end{array}$ $\begin{array}{lll}1.33062800 & -1.06202300 & 3.25364900\end{array}$ $\begin{array}{lll}0.26486300 & -1.30094800 & 3.32288300\end{array}$ $\begin{array}{lll}1.70226700 & -1.40885100 & 2.28498600\end{array}$ $\begin{array}{lll}1.85123800 & -1.62466600 & 4.03628700\end{array}$ $0.98490200 \quad 1.30647600 \quad 5.17394000$ $\begin{array}{llll}1.26898200 & 2.33327100 & 5.42517100\end{array}$ $\begin{array}{lll}-0.10346800 & 1.22671600 & 5.24493400\end{array}$ $\begin{array}{lll}1.42092000 & 0.65121900 & 5.93705100\end{array}$ $3.51553600 \quad 1.07291200 \quad 3.50480100$ $\begin{array}{lll}3.97708200 & 0.39947300 & 4.23645400\end{array}$ $\begin{array}{lll}3.97847400 & 0.87856100 & 2.53381900\end{array}$ $\begin{array}{llll}3.76677500 & 2.09728700 & 3.79406700\end{array}$ $0.88212100-2.73052500-2.59831200$ $\begin{array}{llll}0.04286900 & -2.22221200 & -3.07996300\end{array}$ $\begin{array}{llll}1.77098500 & -2.55887400 & -3.21294300\end{array}$ $\begin{array}{llll}0.67043600 & -3.80623200 & -2.61683000\end{array}$ $\begin{array}{llll}2.47764300 & -3.40214600 & -0.13299500\end{array}$ $\begin{array}{llll}3.36672100 & -3.45844900 & -0.76822900\end{array}$ $\begin{array}{llll}2.79841700 & -3.16263100 & 0.88449800\end{array}$ $\begin{array}{llll}2.02782000 & -4.40210000 & -0.11391400\end{array}$ $\begin{array}{llll}-0.39984100 & -2.52572100 & 0.20677600\end{array}$ $\begin{array}{llll}-0.83914900 & -3.47521800 & -0.12052900\end{array}$ $\begin{array}{lll}-0.15925600 & -2.62465700 & 1.26955300\end{array}$ $\begin{array}{lll}-1.17373200 & -1.75715900 & 0.11411000\end{array}$ $\begin{array}{llll}0.43797600 & 2.71341800 & -2.23426800\end{array}$ $0.61350500 \quad 3.34513700 \quad-3.53296900$ $\begin{array}{llll}1.69016200 & 4.42519500 & -3.41037200\end{array}$ $\begin{array}{llll}1.05418900 & 2.25663300 & -4.50268000\end{array}$ $\begin{array}{lll}-0.71792000 & 3.96748100 & -3.95866000\end{array}$ $\begin{array}{llll}1.39550000 & 5.19544100 & -2.68921400\end{array}$ $\begin{array}{llll}2.64210000 & 3.99477500 & -3.08756600\end{array}$ $\begin{array}{llll}1.84757400 & 4.91697100 & -4.37545700\end{array}$ $\begin{array}{llll}0.31232000 & 1.45677600 & -4.56483100\end{array}$ $\begin{array}{llll}1.18921200 & 2.67775600 & -5.50361200\end{array}$ $2.00440300 \quad 1.82244000-4.18217400$ $\begin{array}{llll}-0.61766700 & 4.46008600 & -4.93132600\end{array}$ $\begin{array}{llll}-1.49962300 & 3.20765100 & -4.04132000\end{array}$ $\begin{array}{llll}-1.04565900 & 4.71913100 & -3.23286500\end{array}$

$\begin{array}{lrrr}\text { TS }_{\text {ad-IA }}\left({ }^{t} \text { BuOH) }\right. & & \\ H=-1170.833493 a u & & \\ \mathrm{U} & 0.37125700 & 1.22162700 & 0.09512400 \\ \mathrm{Si} & 0.15487800 & 4.32560400 & 1.44960000 \\ \mathrm{Si} & 1.95162200 & 2.76018200 & 3.29224200 \\ \mathrm{Si} & -2.34488000 & -0.33339000 & 1.34909700 \\ \mathrm{Si} & -3.28050500 & 1.32714200 & -1.04345200 \\ \mathrm{Si} & 1.16273500 & -1.98954000 & -1.83223000 \\ \mathrm{Si} & 3.47742600 & -0.96828900 & -0.09607300 \\ \mathrm{~N} & 0.94009400 & 2.81047300 & 1.85018600 \\ \mathrm{~N} & -2.00628800 & 0.75692100 & 0.02648400 \\ \mathrm{~N} & 1.93424100 & -0.67734700 & -0.91470800 \\ \mathrm{C} & 4.80529800 & -1.82063600 & -1.15655500 \\ \mathrm{H} & 4.92662300 & -1.34594400 & -2.13493700 \\ \mathrm{H} & 5.76851400 & -1.76693600 & -0.63569700 \\ \mathrm{H} & 4.58216700 & -2.87935800 & -1.31987500 \\ \mathrm{C} & 3.29993800 & -2.03891600 & 1.46555200 \\ \mathrm{H} & 3.07165700 & -3.07748400 & 1.20760800 \\ \mathrm{H} & 4.24163300 & -2.03872700 & 2.02649600\end{array}$




\begin{tabular}{|c|c|c|c|}
\hline 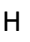 & 2.51434300 & -1.68859100 & 2.14162900 \\
\hline & 4.19854200 & 0.72016500 & 0.39911900 \\
\hline & 4.61028000 & 1.23546400 & -0.47447900 \\
\hline $\mathrm{H}$ & 3.47410800 & 1.39822400 & 0.86404800 \\
\hline $\mathrm{H}$ & 5.01381700 & 0.58429300 & 1.11806200 \\
\hline C & -2.53991800 & 1.92707900 & -2.69103600 \\
\hline & -2.05754100 & 1.10492600 & -3.22837900 \\
\hline & -3.33994100 & 2.31600000 & -3.33125200 \\
\hline & -1.80727000 & 2.73132700 & -2.57080100 \\
\hline C & -4.26975200 & 2.77092300 & -0.29366900 \\
\hline $\mathrm{H}$ & -5.04393600 & 3.10724700 & -0.99318300 \\
\hline $\mathrm{H}$ & -4.76804000 & 2.46693800 & 0.63271300 \\
\hline $\mathrm{H}$ & -3.63738500 & 3.63243900 & -0.05804600 \\
\hline C & -4.55753700 & -0.00694700 & -1.50153400 \\
\hline $\mathrm{H}$ & -4.08222500 & -0.90148700 & -1.91549300 \\
\hline H & -5.16622100 & -0.31099600 & -0.64382000 \\
\hline H & -5.24110200 & 0.39114400 & -2.26040100 \\
\hline C & -0.66240200 & -0.65388300 & 2.23332200 \\
\hline $\mathrm{H}$ & 0.08854600 & -1.17481100 & 1.61743200 \\
\hline $\mathrm{H}$ & -0.22062400 & 0.23777000 & 2.69990600 \\
\hline $\mathrm{H}$ & -0.85589200 & -1.34119400 & 3.06387200 \\
\hline C & -2.96428400 & -2.06033600 & 0.86016300 \\
\hline $\mathrm{H}$ & -2.95541600 & -2.72175400 & 1.73437500 \\
\hline $\mathrm{H}$ & -3.98690100 & -2.03372100 & 0.47496100 \\
\hline $\mathrm{H}$ & -2.32920300 & -2.50934200 & 0.09045400 \\
\hline C & -3.51888200 & 0.35635700 & 2.67017500 \\
\hline $\mathrm{H}$ & -3.60952000 & -0.33300700 & 3.51723100 \\
\hline $\mathrm{H}$ & -3.16595700 & 1.32016200 & 3.04963100 \\
\hline $\mathrm{H}$ & -4.52271600 & 0.50644700 & 2.25878800 \\
\hline C & -0.51262400 & 4.15983300 & -0.34402700 \\
\hline $\mathrm{H}$ & -0.92579900 & 5.13012100 & -0.64060800 \\
\hline $\mathrm{H}$ & 0.24981100 & 3.92435200 & -1.09922500 \\
\hline $\mathrm{H}$ & -1.35342700 & 3.45900300 & -0.42539600 \\
\hline C & -1.37268600 & 4.74263000 & 2.49693800 \\
\hline $\mathrm{H}$ & -2.08876600 & 3.91463600 & 2.48748000 \\
\hline $\mathrm{H}$ & -1.10946700 & 4.94537900 & 3.53875700 \\
\hline $\mathrm{H}$ & -1.88024600 & 5.63083300 & 2.10271300 \\
\hline C & 1.29151100 & 5.84773900 & 1.43391900 \\
\hline $\mathrm{H}$ & 2.17531800 & 5.68050600 & 0.81039000 \\
\hline $\mathrm{H}$ & 0.75215500 & 6.71499500 & 1.03566400 \\
\hline $\mathrm{H}$ & 1.63436900 & 6.10951500 & 2.43990800 \\
\hline C & 2.29736300 & 0.97350100 & 3.84496800 \\
\hline $\mathrm{H}$ & 1.40181300 & 0.47083900 & 4.22335600 \\
\hline $\mathrm{H}$ & 2.72964900 & 0.35505700 & 3.05295900 \\
\hline $\mathrm{H}$ & 3.02589100 & 0.99440400 & 4.66376600 \\
\hline C & 1.15832100 & 3.61765200 & 4.79659900 \\
\hline $\mathrm{H}$ & 1.05014700 & 4.69780600 & 4.65337800 \\
\hline $\mathrm{H}$ & 0.16892000 & 3.20583800 & 5.02027300 \\
\hline $\mathrm{H}$ & 1.79027100 & 3.46925900 & 5.68005300 \\
\hline C & 3.65158300 & 3.58038600 & 3.04079000 \\
\hline $\mathrm{H}$ & 4.21530400 & 3.11281300 & 2.22783600 \\
\hline $\mathrm{H}$ & 3.55776400 & 4.64555100 & 2.81043800 \\
\hline $\mathrm{H}$ & 4.24880500 & 3.49007700 & 3.95590400 \\
\hline C & 2.16695900 & -2.57781500 & -3.33590900 \\
\hline $\mathrm{H}$ & 3.08385000 & -3.10282500 & -3.05678400 \\
\hline $\mathrm{H}$ & 1.55480300 & -3.26954200 & -3.92647200 \\
\hline $\mathrm{H}$ & 2.44304100 & -1.74360200 & -3.98786500 \\
\hline C & 0.81516100 & -3.52673100 & -0.77033400 \\
\hline $\mathrm{H}$ & 1.73852800 & -4.04814600 & -0.49839000 \\
\hline $\mathrm{H}$ & 0.28362300 & -3.28389700 & 0.15493400 \\
\hline $\mathrm{H}$ & 0.19580100 & -4.23376300 & -1.33410400 \\
\hline C & -0.48335200 & -1.36258300 & -2.53391000 \\
\hline & $38<3$ & $-2.18 / 968$ & $-3.03<99000$ \\
\hline
\end{tabular}

$\begin{array}{llll}\mathrm{H} & -1.15784900 & -0.96034400 & -1.76962800 \\ \mathrm{H} & -0.31384500 & -0.58157200 & -3.28284800 \\ \mathrm{O} & 1.66856500 & 1.56837200 & -1.97499800 \\ \mathrm{H} & 1.99364600 & 0.51065500 & -1.72073500 \\ \mathrm{C} & 2.32062200 & 2.30000800 & -3.02579100 \\ \mathrm{C} & 3.30561600 & 1.37688800 & -3.74515700 \\ \mathrm{H} & 2.78413000 & 0.52485500 & -4.19045500 \\ \mathrm{H} & 3.82001600 & 1.91564700 & -4.54718800 \\ \mathrm{H} & 4.06404500 & 0.99592800 & -3.05458200 \\ \mathrm{C} & 1.25072200 & 2.78740500 & -4.00448600 \\ \mathrm{H} & 0.52761300 & 3.43588000 & -3.50036500 \\ \mathrm{H} & 1.70123900 & 3.35849200 & -4.82329400 \\ \mathrm{H} & 0.70713100 & 1.94051800 & -4.43312800 \\ \mathrm{C} & 3.06573500 & 3.48885600 & -2.41301100 \\ \mathrm{H} & 2.37449200 & 4.15678000 & -1.88983300 \\ \mathrm{H} & 3.81552800 & 3.14788600 & -1.69361500 \\ \mathrm{H} & 3.57343100 & 4.07326000 & -3.18754200\end{array}$

\section{Int-IA ('BuOH)}

$H=-868.437936 a u$

$\begin{array}{llll}\text { U } & 0.20254400 & 0.92096800 & 0.37419600\end{array}$

$\begin{array}{lllll}\text { Si } & 0.58345700 & 3.80047600 & 2.09482400\end{array}$

Si $\quad \begin{array}{llll}2.26127200 & 1.55552200 & 3.40957200\end{array}$

Si $\quad 1.06689700 \quad-2.61407700-0.35800200$

Si $\quad 2.72629300 \quad-0.29077200 \quad-1.54680800$

$\begin{array}{lllll}\mathrm{N} & 1.15775000 & 2.16097000 & 2.19123600\end{array}$

N $\quad \begin{array}{llll}\mathrm{N} & 1.47530300 & -0.91519500 & -0.51418200\end{array}$

$\begin{array}{llll}\text { C } & 2.68417700 & -0.92897500 & -3.33465800\end{array}$

H $\quad 1.70147100 \quad-0.77725700 \quad-3.79248500$

H $\quad 3.43137100-0.41617700 \quad-3.95083700$

H $\quad 2.90632600-2.00065400 \quad-3.37211000$

C $\quad 4.49051900-0.50254600 \quad-0.88402000$

$\mathrm{H} \quad 4.75764000-1.56211500 \quad-0.82404200$

H $\quad 5.22250700-0.00848000-1.53296700$

H $\quad 4.58691500 \quad-0.08000800 \quad 0.12111100$

$\begin{array}{lllll}\text { C } & 2.44598000 & 1.62137300 & -1.67065500\end{array}$

H $\quad 1.48889100 \quad 1.91049600 \quad-2.13584100$

$\begin{array}{llll}\mathrm{H} & 2.58419600 & 2.16765800 & -0.72531600\end{array}$

H $\quad 3.21028800 \quad 2.02657500 \quad-2.34282700$

C $\quad-3.31960200 \quad-0.22078000 \quad 2.53684500$

$\begin{array}{llll}\mathrm{H} & -2.75193500 & -1.14710700 & 2.67159600\end{array}$

H $\quad-2.88523200 \quad 0.54465600 \quad 3.18847800$

H $\quad-4.35073500 \quad-0.40179200 \quad 2.86037700$

$\begin{array}{llll}\text { C } & -3.84944900 & -0.85136600 & 0.16030900\end{array}$

$\begin{array}{lllll}\mathrm{H} & -4.90054900 & -1.04976800 & 0.39803100\end{array}$

H $\quad-3.78799100-0.53804200-0.88721000$

$\begin{array}{llll}\text { H } & -3.29152900 & -1.78668500 & 0.26918500\end{array}$

$\begin{array}{lllll}\text { C } & -4.03972300 & 1.54170600 & 0.89721400\end{array}$

$\begin{array}{llll}\mathrm{H} & -5.09745900 & 1.42084200 & 1.15621000\end{array}$

$\begin{array}{llll}\mathrm{H} & -3.61943100 & 2.32333500 & 1.53912400\end{array}$

H $\quad-3.98050100 \quad 1.88075900 \quad-0.14285500$

$\begin{array}{llll}\text { C } & -0.32181200 & 3.98263700 & 0.39287400\end{array}$

$\begin{array}{llll}\text { H } & -0.62247500 & 5.03114500 & 0.28870000\end{array}$

H $\quad 0.32030500 \quad 3.78900500 \quad-0.48121100$

$\mathrm{H} \quad \begin{array}{llll}\mathrm{H} & -1.26013700 & 3.41381300 & 0.31031800\end{array}$

$\begin{array}{llll}\text { C } & -0.70641800 & 4.27526200 & 3.40186600\end{array}$

$\begin{array}{lllll}\mathrm{H} & -0.27266700 & 4.25842100 & 4.40634200\end{array}$

$\mathrm{H} \quad-1.10089500 \quad 5.28209400 \quad 3.22378400$

$\begin{array}{llll}\text { H } & -1.54669200 & 3.57348200 & 3.39146100\end{array}$

$\begin{array}{lllll}\text { C } & 1.95258600 & 5.11521100 & 2.09379700\end{array}$

H $\quad 2.69085900 \quad 4.92228600 \quad 1.30909000$

$\begin{array}{llll}\mathrm{H} & 1.53569300 & 6.11584800 & 1.93344200\end{array}$

$\begin{array}{llll}\text { H } & 2.48198800 & 5.12883700 & 3.05240400\end{array}$ 


$\begin{array}{lrrr}\mathrm{C} & 2.16463100 & -0.34274100 & 3.39759600 \\ \mathrm{H} & 1.16831200 & -0.68979800 & 3.69529600 \\ \mathrm{H} & 2.39607500 & -0.75748000 & 2.40888900 \\ \mathrm{H} & 2.88357500 & -0.77265500 & 4.10381100 \\ \mathrm{C} & 1.83658000 & 2.14404100 & 5.16651400 \\ \mathrm{H} & 1.95586300 & 3.22763700 & 5.27468800 \\ \mathrm{H} & 0.80458300 & 1.88992900 & 5.42879800 \\ \mathrm{H} & 2.49779700 & 1.66783900 & 5.89944900 \\ \mathrm{C} & 4.06415400 & 2.04698700 & 3.05836600 \\ \mathrm{H} & 4.74383700 & 1.60065100 & 3.79327200 \\ \mathrm{H} & 4.37779100 & 1.70964000 & 2.06477800 \\ \mathrm{H} & 4.19768700 & 3.13293000 & 3.09752500 \\ \mathrm{C} & 0.06259800 & -3.24796900 & -1.84273700 \\ \mathrm{H} & -0.85475800 & -2.66293200 & -1.97039600 \\ \mathrm{H} & 0.63372100 & -3.18037900 & -2.77403500 \\ \mathrm{H} & -0.22617300 & -4.29605500 & -1.70316800 \\ \mathrm{C} & 2.59496300 & -3.73028100 & -0.16914900 \\ \mathrm{H} & 3.22456200 & -3.71307400 & -1.06567900 \\ \mathrm{H} & 3.21058900 & -3.41773100 & 0.68044500 \\ \mathrm{H} & 2.29350200 & -4.77052200 & -0.00129300 \\ \mathrm{C} & -0.01632200 & -2.82679400 & 1.18906300 \\ \mathrm{H} & -0.38226400 & -3.85671000 & 1.26576300 \\ \mathrm{H} & 0.54002100 & -2.60478300 & 2.10549400 \\ \mathrm{H} & -0.89633500 & -2.17331100 & 1.15170600 \\ \mathrm{C} & -3.25833000 & 0.23230100 & 1.07209400 \\ \mathrm{O} & -1.91628400 & 0.45162200 & 0.71182000\end{array}$

\section{Int-IIA ('BuOH)}

$H=-799.510442 a u$

$\begin{array}{llll}\text { U } & 0.38051200 & 0.80409000 & 0.63809400 \\ \mathrm{Si} & 1.43924700 & -2.54185000 & -0.04042800 \\ \mathrm{Si} & 2.30162700 & -0.17792900 & -1.96965400 \\ \mathrm{~N} & 1.52777900 & -0.90305600 & -0.61065500 \\ \mathrm{C} & 1.64059700 & -0.72450200 & -3.66119600 \\ \mathrm{H} & 0.55563900 & -0.59236400 & -3.72347700 \\ \mathrm{H} & 2.10335900 & -0.15668900 & -4.47620400 \\ \mathrm{H} & 1.85378400 & -1.78482100 & -3.83199400 \\ \mathrm{C} & 4.19392000 & -0.30872300 & -1.98739600 \\ \mathrm{H} & 4.50615900 & -1.35253300 & -2.09717600 \\ \mathrm{H} & 4.62784800 & 0.25710500 & -2.81951400 \\ \mathrm{H} & 4.62573000 & 0.06754800 & -1.05452500 \\ \mathrm{C} & 1.91565600 & 1.72635000 & -1.85826500 \\ \mathrm{H} & 0.85083300 & 1.98959500 & -1.97307700 \\ \mathrm{H} & 2.33916900 & 2.23173300 & -0.97465000 \\ \mathrm{H} & 2.40525700 & 2.21330800 & -2.70859500 \\ \mathrm{C} & -3.56564800 & 0.23715700 & 2.10372200 \\ \mathrm{H} & -3.06821000 & -0.67382300 & 2.45344600 \\ \mathrm{H} & -3.23410400 & 1.06925600 & 2.73393900 \\ \mathrm{H} & -4.64544800 & 0.11099800 & 2.24124200 \\ \mathrm{C} & -3.64648700 & -0.67225500 & -0.23974200 \\ \mathrm{H} & -4.72939600 & -0.83283700 & -0.19065500 \\ \mathrm{H} & -3.37204400 & -0.48975200 & -1.28386600 \\ \mathrm{H} & -3.14936600 & -1.59189600 & 0.08610800 \\ \mathrm{C} & -3.88905100 & 1.79695800 & 0.15923300 \\ \mathrm{H} & -4.98078600 & 1.72443400 & 0.21849800 \\ \mathrm{H} & -3.56815100 & 2.64463200 & 0.77420900 \\ \mathrm{H} & -3.61560300 & 2.00476900 & -0.88074500 \\ \mathrm{C} & 0.58017700 & -3.72699500 & -1.24933200 \\ \mathrm{H} & -0.41220000 & -3.35579000 & -1.52441700 \\ \mathrm{H} & 1.16047100 & -3.84218000 & -2.17107300 \\ \mathrm{H} & 0.46024800 & -4.72265800 & -0.80735400 \\ \mathrm{C} & 3.12646700 & -3.28231100 & 0.41771500 \\ \mathrm{H} & 3.76233000 & -3.39987100 & -0.46627900\end{array}$

$\begin{array}{lll}3.65767400 & -2.63914600 & 1.12663600\end{array}$ $3.01147800-4.27110100 \quad 0.87610600$ $\begin{array}{lll}0.38286900 & -2.46561900 & 1.55181900\end{array}$ $\begin{array}{llll}0.28009000 & -3.45956600 & 2.00089300\end{array}$ $\begin{array}{llll}0.83967400 & -1.83018400 & 2.32332100\end{array}$ $\begin{array}{lll}-0.63883600 & -2.11326900 & 1.35383300\end{array}$ $\begin{array}{lll}-3.20523600 & 0.50803400 & 0.63663500\end{array}$ $\begin{array}{lll}-1.81528800 & 0.66965000 & 0.52325700\end{array}$ $\begin{array}{lll}1.27453500 & 1.29041200 & 2.59300800\end{array}$ $\begin{array}{lll}1.80214600 & 1.48056400 & 3.87984900\end{array}$ $\begin{array}{lll}0.95231200 & 0.70658600 & 4.89677900\end{array}$ $\begin{array}{lll}3.24908500 & 0.96873700 & 3.90718400\end{array}$ $\begin{array}{lll}1.77073600 & 2.97983500 & 4.20835700\end{array}$ $\begin{array}{lll}-0.08450500 & 1.05741100 & 4.86840700\end{array}$ $\begin{array}{lll}0.95770000 & -0.36263400 & 4.65988500\end{array}$ $\begin{array}{llll}1.32935400 & 0.83093800 & 5.91810800\end{array}$ $\begin{array}{lll}3.85475500 & 1.51175400 & 3.17383000\end{array}$ $\begin{array}{lll}3.70888500 & 1.09581800 & 4.89381500\end{array}$ $\begin{array}{lll}3.27759000 & -0.09514700 & 3.64947100\end{array}$ $\begin{array}{llll}2.17933700 & 3.18669100 & 5.20387600\end{array}$ $\begin{array}{llll}2.36033800 & 3.53892700 & 3.47378200\end{array}$ $\begin{array}{llll}0.74106700 & 3.35142400 & 4.17607000\end{array}$

$\begin{array}{lrrr}\text { ad-B ( }{ }^{t} \text { BuOH) } & & \\ \mathrm{H}=-1170.831886 a u & & \\ \mathrm{U} & 0.00605700 & 0.14314200 & -0.00578000 \\ \mathrm{Si} & -0.22657000 & 3.16381100 & 1.53102800 \\ \mathrm{Si} & 1.37655400 & 1.42177600 & 3.40118600 \\ \mathrm{Si} & -2.85179200 & -1.31430400 & 1.22180700 \\ \mathrm{Si} & -3.56032600 & 0.32437000 & -1.25292400 \\ \mathrm{Si} & 0.79332900 & -3.19964900 & -1.45950800 \\ \mathrm{Si} & 3.19109700 & -1.72597600 & -0.26782900 \\ \mathrm{~N} & 0.50764100 & 1.61275300 & 1.87890900 \\ \mathrm{~N} & -2.37335300 & -0.29209800 & -0.11572400 \\ \mathrm{~N} & 1.50584700 & -1.73034400 & -0.78193900 \\ \mathrm{C} & 4.45254000 & -2.04232100 & -1.66167300 \\ \mathrm{H} & 4.25394500 & -1.43047600 & -2.54803900 \\ \mathrm{H} & 5.45644900 & -1.78480600 & -1.30362800 \\ \mathrm{H} & 4.47369100 & -3.08758400 & -1.97968700 \\ \mathrm{C} & 3.58227300 & -2.99256700 & 1.09435400 \\ \mathrm{H} & 3.53215600 & -4.01412100 & 0.70244300 \\ \mathrm{H} & 4.59564200 & -2.83998600 & 1.48267300 \\ \mathrm{H} & 2.88513900 & -2.92528900 & 1.93504300 \\ \mathrm{C} & 3.61802100 & 0.01818300 & 0.36757000 \\ \mathrm{H} & 3.67335200 & 0.73748600 & -0.45643600 \\ \mathrm{H} & 2.91814200 & 0.41916100 & 1.10751900 \\ \mathrm{H} & 4.60299300 & 0.00040100 & 0.84757200 \\ \mathrm{C} & -2.69135300 & 0.85930000 & -2.85919900 \\ \mathrm{H} & -2.16870400 & 0.01798800 & -3.32573800 \\ \mathrm{H} & -3.43211400 & 1.23173100 & -3.57573500 \\ \mathrm{H} & -1.97029500 & 1.66719600 & -2.69830500 \\ \mathrm{C} & -4.49756500 & 1.84414800 & -0.59399900 \\ \mathrm{H} & -5.20942100 & 2.21062000 & -1.34286600 \\ \mathrm{H} & -5.06264800 & 1.60070000 & 0.31131900 \\ \mathrm{H} & -3.82095500 & 2.66801300 & -0.34578100 \\ \mathrm{C} & -4.88199500 & -0.94434600 & -1.76664000 \\ \mathrm{H} & -4.43373800 & -1.86597200 & -2.15046400 \\ \mathrm{H} & -5.54766300 & -1.20971900 & -0.93885400 \\ \mathrm{H} & -5.50575300 & -0.51800000 & -2.56096700 \\ \mathrm{C} & -1.26492700 & -1.60902300 & 2.25350000 \\ \mathrm{H} & -0.46123000 & -2.14636700 & 1.72698600 \\ \mathrm{H} & -0.87096700 & -0.67865700 & 2.68232300 \\ \mathrm{H} & -1.51344900 & -2.24983200 & 3.10647500\end{array}$


C $\quad-3.50479400 \quad-3.03191800 \quad 0.74021100$

H $\quad-3.56807300 \quad-3.66991300 \quad 1.62954100$

$\begin{array}{lllll}\mathrm{H} & -4.50482000 & -2.97611400 & 0.30175300\end{array}$

H $\quad-2.85037100 \quad-3.52604700 \quad 0.01614200$

C $\quad-4.11807700 \quad-0.54146500 \quad 2.40781200$

H $\quad-4.29122000-1.19978000 \quad 3.26694200$

$\begin{array}{lllll}H & -3.77478700 & 0.42636800 & 2.78615000\end{array}$

$\begin{array}{llll}\text { H } & -5.08343300 & -0.38650800 & 1.91458100\end{array}$

$\begin{array}{llll}\text { C } & -0.59615200 & 3.20832700 & -0.35708800\end{array}$

H $\quad-0.93105400 \quad 4.22041200 \quad-0.61049600$

$\begin{array}{llll}H & 0.29264000 & 3.02525000 & -0.97550400\end{array}$

$\begin{array}{llll}\mathrm{H} & -1.41879000 & 2.55673300 & -0.68198300\end{array}$

C $\quad \begin{array}{llll}-1.89354500 & 3.46166500 & 2.38659400\end{array}$

$\begin{array}{llll}H & -2.59683400 & 2.65753400 & 2.14834400\end{array}$

$\begin{array}{llll}\mathrm{H} & -1.78261900 & 3.49906900 & 3.47419100\end{array}$

$\mathrm{H}$

C
$0.13495900 \quad 1.25874300 \quad-0.07351300$ $\begin{array}{llll}0.63222000 & 3.62797100 & 1.99381700\end{array}$ $\begin{array}{lll}1.73053300 & 1.02699000 & 3.32209400\end{array}$ $\begin{array}{lll}-3.16563100 & 0.83057900 & 1.47696800\end{array}$ $\begin{array}{llll}-2.91068000 & 0.28129300 & -1.49314600\end{array}$ $0.97083800-2.26010000-0.72493300$ $\begin{array}{llll}3.22197200 & -0.22365900 & -0.94263400\end{array}$ $\begin{array}{lll}0.87544700 & 1.85350100 & 2.00577800\end{array}$ $\begin{array}{lll}-2.10408200 & 0.67133800 & 0.04516500\end{array}$ $\begin{array}{llll}1.51328700 & -0.56568100 & -0.60277100\end{array}$ $3.72059100-0.71347000-2.70946000$ $\begin{array}{llll}3.05008700 & -0.27594200 & -3.45532600\end{array}$ $\begin{array}{llll}4.73959000 & -0.37252800 & -2.92598300\end{array}$ $\begin{array}{llll}3.70448800 & -1.80023400 & -2.84382900\end{array}$ $\begin{array}{llll}4.46509200 & -1.04761000 & 0.23658100\end{array}$ $\begin{array}{llll}4.67153200 & -2.08643500 & -0.03099100\end{array}$ $\begin{array}{llll}5.41366200 & -0.49975500 & 0.18908800\end{array}$ $\begin{array}{llll}4.12228100 & -1.02954300 & 1.27469000\end{array}$ $\begin{array}{llll}3.50395500 & 1.64776900 & -0.74864500\end{array}$ $\begin{array}{llll}2.88519500 & 2.24815800 & -1.42109500\end{array}$ $\begin{array}{llll}3.33404600 & 1.98542900 & 0.27982600\end{array}$ $\begin{array}{llll}4.55052800 & 1.86969100 & -0.98690600\end{array}$ $\begin{array}{lll}-1.64827700 & 0.44690400 & -2.90514000\end{array}$ $\begin{array}{lll}-0.72995600 & -0.13598300 & -2.77080700\end{array}$ $\begin{array}{lll}-2.11807400 & 0.07036100 & -3.82115300\end{array}$ $\begin{array}{llll}-1.37198900 & 1.48796000 & -3.07921100\end{array}$ $\begin{array}{llll}-4.31616300 & 1.48875500 & -1.91034300\end{array}$ $\begin{array}{llll}-4.70129100 & 1.27497600 & -2.91406500\end{array}$ $\begin{array}{llll}-5.15670700 & 1.41283300 & -1.21429600\end{array}$ $\begin{array}{llll}-3.96160200 & 2.52437400 & -1.89855100\end{array}$ $\begin{array}{llll}-3.59276500 & -1.48563300 & -1.61885300\end{array}$ $\begin{array}{lll}-4.40299000 & -1.68343300 & -0.91399900\end{array}$ $\begin{array}{lll}-3.98892800 & -1.63461800 & -2.63051300\end{array}$ $\begin{array}{llll}-2.81178900 & -2.23328700 & -1.45545400\end{array}$ $\begin{array}{lll}-2.22478100 & 0.39931300 & 3.05900000\end{array}$ $\begin{array}{lll}-1.93915000 & -0.65763500 & 3.06221600\end{array}$ $\begin{array}{lll}-1.32092800 & 1.00190100 & 3.17589500\end{array}$ $\begin{array}{lll}-2.87008800 & 0.57393900 & 3.92742100\end{array}$ $\begin{array}{lll}-4.66319100 & -0.34039700 & 1.45048200\end{array}$ $\begin{array}{lll}-5.22820200 & -0.17997400 & 2.37648100\end{array}$ $\begin{array}{lll}-5.34901600 & -0.16294500 & 0.61755900\end{array}$ $\begin{array}{lll}-4.35795100 & -1.39091300 & 1.42945700\end{array}$ $\begin{array}{lll}-3.84866800 & 2.59513100 & 1.62436200\end{array}$ $\begin{array}{lll}-4.48232400 & 2.68384400 & 2.51428400\end{array}$ $\begin{array}{lll}-3.04912700 & 3.33659600 & 1.71089700\end{array}$ $\begin{array}{lll}-4.45603100 & 2.86009600 & 0.75362400\end{array}$ $\begin{array}{lll}-0.11972900 & 3.89944800 & 0.28584900\end{array}$ $\begin{array}{llll}0.24476300 & 4.88007800 & -0.05026000\end{array}$ $\begin{array}{llll}0.26750700 & 3.42684300 & -1.16313800\end{array}$ $\begin{array}{lll}-1.21474900 & 3.98903900 & 0.34858200\end{array}$ $\begin{array}{lll}-0.49826800 & 4.23907900 & 3.39308500\end{array}$ $\begin{array}{lll}-0.01061900 & 4.19948500 & 4.37142200\end{array}$ $\begin{array}{lll}-0.77474300 & 5.28191200 & 3.19918100\end{array}$ $\begin{array}{lll}-1.42024400 & 3.65381800 & 3.45438600\end{array}$ $\begin{array}{llll}2.26007800 & 4.59874200 & 2.12355600\end{array}$ $\begin{array}{lll}2.98291300 & 4.28810800 & 1.36299600\end{array}$ $\begin{array}{lll}2.05872600 & 5.66685200 & 1.98009900\end{array}$ $\begin{array}{llll}2.72985700 & 4.48419500 & 3.10557100\end{array}$ $\begin{array}{lll}1.47628300 & -0.84667400 & 3.20161000\end{array}$ $\begin{array}{llll}0.44277300 & -1.12412400 & 3.42984500\end{array}$ $\begin{array}{lll}1.72102800 & -1.21614700 & 2.20172400\end{array}$ $\begin{array}{llll}2.12483900 & -1.35882500 & 3.92114700\end{array}$ $\begin{array}{lll}1.12656000 & 1.57399400 & 5.03865000\end{array}$ 


$\begin{array}{lrrr}\text { H } & 1.42425600 & 2.60159300 & 5.26889600 \\ \mathrm{H} & 0.03987900 & 1.50241800 & 5.13824800 \\ \mathrm{H} & 1.57677100 & 0.92510300 & 5.79899500 \\ \mathrm{C} & 3.60132300 & 1.35917400 & 3.29374500 \\ \mathrm{H} & 4.08257500 & 0.80456600 & 4.10799900 \\ \mathrm{H} & 4.06679500 & 1.04134400 & 2.35718800 \\ \mathrm{H} & 3.82792500 & 2.41945000 & 3.43774900 \\ \mathrm{C} & 0.47271100 & -2.74058700 & -2.49742100 \\ \mathrm{H} & -0.46441400 & -2.27781300 & -2.81826800 \\ \mathrm{H} & 1.24787300 & -2.46489600 & -3.21919800 \\ \mathrm{H} & 0.33571700 & -3.82681900 & -2.55454700 \\ \mathrm{C} & 2.28334300 & -3.53659100 & -0.21327100 \\ \mathrm{H} & 3.12974300 & -3.57931100 & -0.90517900 \\ \mathrm{H} & 2.66981000 & -3.36274600 & 0.79463200 \\ \mathrm{H} & 1.80646200 & -4.52405800 & -0.22083700 \\ \mathrm{C} & -0.50837500 & -2.57028000 & 0.42364000 \\ \mathrm{H} & -0.96315000 & -3.53590500 & 0.17394000 \\ \mathrm{H} & -0.18065500 & -2.61867400 & 1.46651700 \\ \mathrm{H} & -1.28579900 & -1.80441200 & 0.34745700 \\ \mathrm{O} & 0.51332400 & 2.76148000 & -2.01099100 \\ \mathrm{C} & 0.80306600 & 3.45289200 & -3.24727300 \\ \mathrm{C} & 1.88841300 & 4.50269800 & -2.98759900 \\ \mathrm{C} & 1.29068000 & 2.42122600 & -4.25971700 \\ \mathrm{C} & -0.47528300 & 4.14163500 & -3.73489400 \\ \mathrm{H} & 1.55678300 & 5.23356100 & -2.24358500 \\ \mathrm{H} & 2.80933700 & 4.03813400 & -2.62544700 \\ \mathrm{H} & 2.12148600 & 5.04394200 & -3.91035600 \\ \mathrm{H} & 0.53340500 & 1.65230300 & -4.43363100 \\ \mathrm{H} & 1.51379200 & 2.90411300 & -5.21674200 \\ \mathrm{H} & 2.20316900 & 1.93475400 & -3.90424600 \\ \mathrm{H} & -0.28093200 & 4.70329400 & -4.65458800 \\ \mathrm{H} & -1.26391600 & 3.41418200 & -3.94553800 \\ \mathrm{H} & -0.84773900 & 4.84297500 & -2.98163300\end{array}$

$\begin{array}{lrrr}\text { Int-IB ('BuOH) } & & \\ H=-1175.959252 a u & & \\ \mathrm{U} & 0.26198300 & 1.15830600 & -0.21257400 \\ \mathrm{Si} & 0.38656100 & 3.81726500 & 2.09399600 \\ \mathrm{Si} & 1.95788700 & 1.38432400 & 3.03475700 \\ \mathrm{Si} & -3.08195700 & 0.77367500 & 1.25159400 \\ \mathrm{Si} & -2.79750000 & 0.30620700 & -1.74145000 \\ \mathrm{Si} & 0.81511400 & -2.45268300 & -0.56171200 \\ \mathrm{Si} & 3.18833900 & -0.60495300 & -1.05494800 \\ \mathrm{~N} & 0.90718300 & 2.14049400 & 1.80616000 \\ \mathrm{~N} & -2.02581600 & 0.68880400 & -0.18442500 \\ \mathrm{~N} & 1.48000100 & -0.79724500 & -0.59813000 \\ \mathrm{C} & 3.55774300 & -1.29099700 & -2.78598400 \\ \mathrm{H} & 2.88720600 & -0.85808300 & -3.53499000 \\ \mathrm{H} & 4.58802600 & -1.05564300 & -3.07634900 \\ \mathrm{H} & 3.44794500 & -2.37950500 & -2.82404800 \\ \mathrm{C} & 4.43408400 & -1.38320000 & 0.15170100 \\ \mathrm{H} & 4.46181100 & -2.47334800 & 0.09553900 \\ \mathrm{H} & 5.43552800 & -1.01418900 & -0.10029200 \\ \mathrm{H} & 4.23030000 & -1.10313400 & 1.18917300 \\ \mathrm{C} & 3.61766900 & 1.24785900 & -1.08460000 \\ \mathrm{H} & 3.15389900 & 1.76539500 & -1.92490100 \\ \mathrm{H} & 3.35423000 & 1.78521600 & -0.16569900 \\ \mathrm{H} & 4.70435700 & 1.34137500 & -1.19298500 \\ \mathrm{C} & -1.49023800 & 0.37193300 & -3.12138500 \\ \mathrm{H} & -0.61300100 & -0.26131600 & -2.94495100 \\ \mathrm{H} & -1.95611000 & 0.00226200 & -4.04219500 \\ \mathrm{H} & -1.14334600 & 1.38848800 & -3.31123600 \\ \mathrm{C} & -4.15132900 & 1.55093300 & -2.21160800\end{array}$

\begin{abstract}
$\begin{array}{lll}-4.52821700 & 1.33485600 & -3.21785800\end{array}$ $\begin{array}{lll}-5.00364800 & 1.50633700 & -1.52631800\end{array}$ $\begin{array}{llll}-3.76755000 & 2.57603000 & -2.20682600\end{array}$ $-3.55312800-1.43561800-1.84589400$ $-4.50160300-1.52284800-1.31115500$ $\begin{array}{lll}-3.74677900 & -1.66949500 & -2.89955200\end{array}$ $\begin{array}{llll}-2.87583900 & -2.20065500 & -1.45574800\end{array}$ $\begin{array}{lll}-2.08828600 & 0.40015300 & 2.82267700\end{array}$ $\begin{array}{lll}-1.78473100 & -0.65121800 & 2.84973800\end{array}$ $\begin{array}{lll}-1.19316900 & 1.02184700 & 2.91410500\end{array}$ $\begin{array}{lll}-2.71773100 & 0.58504500 & 3.70077500\end{array}$ $\begin{array}{lll}-4.50512100 & -0.48588900 & 1.24038800\end{array}$ $\begin{array}{lll}-5.01731000 & -0.41784900 & 2.20752400\end{array}$ $\begin{array}{lll}-5.24858900 & -0.28288400 & 0.46424100\end{array}$ $\begin{array}{lll}-4.15304000 & -1.51456500 & 1.12251300\end{array}$ $\begin{array}{lll}-3.90701400 & 2.47429700 & 1.44813900\end{array}$ $\begin{array}{lll}-4.60974500 & 2.44574100 & 2.28918400\end{array}$ $\begin{array}{lll}-3.18811900 & 3.27297300 & 1.64771700\end{array}$ $\begin{array}{lll}-4.47219000 & 2.74887300 & 0.55224600\end{array}$ $\begin{array}{lll}-0.62762300 & 4.42129700 & 0.60374600\end{array}$ $\begin{array}{lll}-1.07477800 & 5.38630400 & 0.86862700\end{array}$ $\begin{array}{llll}-0.00622700 & 4.57239700 & -0.27935900\end{array}$ $\begin{array}{lll}-1.45911100 & 3.76171600 & 0.32835400\end{array}$ $\begin{array}{lll}-0.75177500 & 4.05078900 & 3.59779600\end{array}$ $\begin{array}{lll}-0.22915800 & 3.95143700 & 4.55115700\end{array}$ $\begin{array}{lll}-1.17711800 & 5.06066100 & 3.55732400\end{array}$ $\begin{array}{lll}-1.58361300 & 3.34090900 & 3.59247200\end{array}$ $\begin{array}{lll}1.85244700 & 5.00910800 & 2.28693500\end{array}$ $\begin{array}{llll}2.53584000 & 4.93689100 & 1.43491900\end{array}$ $\begin{array}{lll}1.49430600 & 6.04328500 & 2.34693700\end{array}$ $\begin{array}{lll}2.42611700 & 4.80834100 & 3.19759400\end{array}$ $\begin{array}{lll}1.74318500 & -0.49897000 & 3.00923500\end{array}$ $\begin{array}{lll}0.74625000 & -0.78066800 & 3.36264200\end{array}$ $\begin{array}{lll}1.89074900 & -0.92510500 & 2.01290100\end{array}$ $\begin{array}{llll}2.47599300 & -0.95714600 & 3.68321400\end{array}$ $\begin{array}{lll}1.57175100 & 1.91355100 & 4.81959200\end{array}$ $\begin{array}{lll}1.79875400 & 2.96526100 & 5.01839700\end{array}$ $\begin{array}{llll}0.52933200 & 1.72608000 & 5.09206900\end{array}$ $\begin{array}{lll}2.20257400 & 1.31434500 & 5.48708100\end{array}$ $\begin{array}{lll}3.79627300 & 1.78558500 & 2.76693200\end{array}$ $\begin{array}{llll}4.38436900 & 1.38339200 & 3.60017300\end{array}$ $\begin{array}{lll}4.19465200 & 1.35283800 & 1.84559600\end{array}$ $\begin{array}{llll}3.96689300 & 2.86581800 & 2.73099500\end{array}$ $\begin{array}{llll}0.19576800 & -3.03009000 & -2.26271500\end{array}$ $\begin{array}{lll}0.66671200 & -2.45991000 & -2.61691200\end{array}$ $\begin{array}{llll}0.98196200 & -2.95449400 & -3.01996400\end{array}$ $-0.10799400-4.08180900-2.20228200$ $\begin{array}{lll}2.05795000 & -3.77589800 & 0.00159600\end{array}$ $\begin{array}{llll}2.86940300 & -3.93754400 & -0.71439600\end{array}$ $\begin{array}{llll}2.49697700 & -3.54931300 & 0.97714000\end{array}$ $\begin{array}{lll}1.51429900 & -4.72381700 & 0.09397600\end{array}$ $\begin{array}{lll}-0.62595300 & -2.54743800 & 0.66583800\end{array}$ $\begin{array}{lll}-1.11804900 & -3.52275900 & 0.57744600\end{array}$ $\begin{array}{lll}-0.26782900 & -2.44874300 & 1.69532300\end{array}$ $\begin{array}{lll}-1.37797900 & -1.77444800 & 0.48275600\end{array}$ $\begin{array}{llll}0.65726400 & 2.54849800 & -1.79674100\end{array}$ $\begin{array}{llll}0.92215900 & 3.46324900 & -2.85152600\end{array}$ $\begin{array}{llll}1.98734600 & 4.46975700 & -2.39670300\end{array}$ $\begin{array}{lll}1.43165600 & 2.69347500 & -4.07750800\end{array}$ $\begin{array}{llll}-0.36987200 & 4.20849200 & -3.21322300\end{array}$ $\begin{array}{llll}1.63761500 & 5.05244000 & -1.53963300\end{array}$ $\begin{array}{llll}2.90799500 & 3.95735600 & -2.10508400\end{array}$ $\begin{array}{llll}2.22678200 & 5.16983300 & -3.20427100\end{array}$
\end{abstract}




$\begin{array}{lrrr}H & 0.70285800 & 1.94378200 & -4.39687900 \\ H & 1.60896500 & 3.37610500 & -4.91549600 \\ H & 2.37253200 & 2.18066200 & -3.85789400 \\ H & -0.18664800 & 4.93378100 & -4.01318600 \\ H & -1.14009900 & 3.51323600 & -3.55944000 \\ H & -0.76396300 & 4.74734600 & -2.34748100\end{array}$

\section{Int-IIB ('BuOH)}

$H=-1107.056407 \mathrm{au}$

$\begin{array}{llll}\text { U } & 0.52308800 & 1.15579800 & 0.01442500\end{array}$

Si $\quad \begin{array}{llll}1.30494200 & 4.18272300 & 1.90292200\end{array}$

Si $\quad \begin{array}{llll}1.37662600 & 1.46308200 & 3.33460700\end{array}$

Si $\quad 1.12050900-2.30105600-1.01708600$

Si $\quad 3.46416600 \quad-0.30993700 \quad-1.00010000$

$\begin{array}{lllll}\mathrm{N} & 1.22630300 & 2.41289000 & 1.85788200\end{array}$

N $\quad \begin{array}{llll}\mathrm{N} & 1.78721800 & -0.70586200 & -0.63216300\end{array}$

C $\quad 3.93212300 \quad-0.63181000 \quad-2.81140300$

H $\quad 3.26329700 \quad-0.08677900-3.48540000$

H $\quad 4.95686900-0.29810600-3.01029600$

H $\quad 3.87557000-1.69343400-3.07062700$

$\begin{array}{lllll}\text { C } & 4.72372400 & -1.17325100 & 0.12651500\end{array}$

H $\quad 4.75312900-2.25360300 \quad-0.03876700$

H $\quad 5.72949100-0.77975000 \quad-0.06101900$

$\mathrm{H} \quad 4.48803900-1.00044100 \quad 1.18176500$

$\begin{array}{llll}\text { C } & 3.68450800 & 1.56417200 & -0.70390700\end{array}$

H $\quad 3.09736800 \quad 2.18753700 \quad-1.38951500$

$\begin{array}{lllll}\mathrm{H} & 3.47479700 & 1.87801700 & 0.32695300\end{array}$

H $\quad 4.73552200 \quad 1.81311300 \quad-0.88962100$

$\begin{array}{lllll}\text { C } & 2.07025100 & 4.84934300 & 0.30052600\end{array}$

$\begin{array}{lllll}\text { H } & 2.01468700 & 5.94380100 & 0.28848400\end{array}$

H $\quad 3.12402700 \quad 4.56543000 \quad 0.21568600$

H $\quad \begin{array}{llll}1.54572500 & 4.47518400 & -0.58355000\end{array}$

$\begin{array}{lllll}\text { C } & -0.43582400 & 4.92569700 & 2.07120300\end{array}$

$\mathrm{H} \quad-0.92238100 \quad 4.59148800 \quad 2.99331000$

$\begin{array}{llll}\mathrm{H} & -0.39554200 & 6.02079200 & 2.09029600\end{array}$

H $\quad-1.07480200 \quad 4.63301500 \quad 1.23076500$

$\begin{array}{llll}\text { C } & 2.37003800 & 4.86229300 & 3.32263700\end{array}$

$\begin{array}{llll}\mathrm{H} & 3.41102900 & 4.53796900 & 3.22879900\end{array}$

$\begin{array}{llll}\mathrm{H} & 2.35962800 & 5.95758300 & 3.27841900\end{array}$

H $\quad 2.00883700 \quad 4.57243800 \quad 4.31370300$

$\begin{array}{llll}\text { C } & 0.93220300 & -0.34093100 & 2.89468000\end{array}$

$\begin{array}{llll}\mathrm{H} & -0.11301200 & -0.46595100 & 2.58596600\end{array}$

H $\quad \begin{array}{llll}1.58224900 & -0.78556400 & 2.13011500\end{array}$

$\begin{array}{llll}\text { H } & 1.05912000 & -0.95207500 & 3.79546400\end{array}$

$\begin{array}{lllll}\text { C } & 0.17187400 & 1.99830100 & 4.70119900\end{array}$

$\mathrm{H} \quad 0.37583000 \quad 3.01202700 \quad 5.05931500$

$\begin{array}{llll}\mathrm{H} & -0.86057500 & 1.97289200 & 4.33771500\end{array}$

$\begin{array}{llll}\text { H } & 0.24362100 & 1.32365700 & 5.56185400\end{array}$

$\begin{array}{llll}\text { C } & 3.13533700 & 1.42568100 & 4.04611600\end{array}$

$\begin{array}{llll}\mathrm{H} & 3.19510600 & 0.70527200 & 4.87003900\end{array}$

$\begin{array}{llll}\mathrm{H} & 3.86124600 & 1.12449600 & 3.28382400\end{array}$

$\begin{array}{llll}\mathrm{H} & 3.44130400 & 2.40097600 & 4.43426600\end{array}$

C $\quad 0.30712500 \quad-2.28043700 \quad-2.73380800$

H $\quad-0.49894900-1.53966800-2.77735700$

$\mathrm{H} \quad 1.03398700-2.02858900-3.51282300$

H $\quad-0.12550700 \quad-3.25684900 \quad-2.97966800$

C $\quad 2.41251700 \quad-3.69493800 \quad-0.99549900$

$\mathrm{H} \quad 3.21228800 \quad-3.55756400 \quad-1.72931500$

H $\quad 2.86998000 \quad-3.80022200-0.00687200$

H $\quad \begin{array}{llll}\text { H } & 1.91326800 & -4.64220700 & -1.23050400\end{array}$

C $\quad-0.20433300 \quad-2.78429000 \quad 0.25137500$

H $\quad-0.70536200 \quad-3.70616700 \quad-0.06500200$

$\begin{array}{llll}\text { H } & 0.23628100 & -2.96295000 & 1.23730400\end{array}$ $\begin{array}{lll}-0.96518600 & -2.00506600 & 0.35562500\end{array}$ $\begin{array}{lll}0.32597000 & 2.44800000 & -1.67463200\end{array}$ $\begin{array}{lll}-0.00896300 & 3.00099200 & -2.93565900\end{array}$ $\begin{array}{llll}1.27640400 & 3.48141000 & -3.61735500\end{array}$ $\begin{array}{lll}-0.68357400 & 1.92047100 & -3.78693100\end{array}$ $\begin{array}{lll}-0.96499000 & 4.17704300 & -2.71237600\end{array}$ $\begin{array}{llll}1.78028400 & 4.23291300 & -3.00205500\end{array}$ $\begin{array}{lll}1.96387000 & 2.64334900 & -3.76985100\end{array}$ $\begin{array}{llll}1.06069900 & 3.92737000 & -4.59421400\end{array}$ $\begin{array}{lll}-1.59890300 & 1.56682900 & -3.30125300\end{array}$ $\begin{array}{lll}-0.95094000 & 2.30433000 & -4.77728000\end{array}$ $\begin{array}{lll}-0.01106300 & 1.06714300 & -3.92101300\end{array}$ $\begin{array}{llll}-1.25030200 & 4.64101500 & -3.66257100\end{array}$ $\begin{array}{lll}-1.87636300 & 3.83593800 & -2.21112500\end{array}$ $\begin{array}{llll}-0.49417100 & 4.94008200 & -2.08535300\end{array}$ $\begin{array}{llll}-1.49238900 & 0.54661300 & 0.37085200\end{array}$ $\begin{array}{lll}-2.89491700 & 0.50580300 & 0.56811600\end{array}$ $\begin{array}{lll}-3.18412800 & -0.23411000 & 1.87829500\end{array}$ $\begin{array}{lll}-3.42573100 & 1.94078800 & 0.64443200\end{array}$ $-3.53177300-0.23481600-0.61222000$ $\begin{array}{lll}-2.78542800 & -1.25244300 & 1.84013000\end{array}$ $\begin{array}{lll}-2.72002500 & 0.28676000 & 2.72172100\end{array}$ $\begin{array}{lll}-4.26137500 & -0.29518900 & 2.06644400\end{array}$ $\begin{array}{lll}-3.21674200 & 2.47606200 & -0.28754600\end{array}$ $\begin{array}{lll}-4.50844900 & 1.95532000 & 0.80919700\end{array}$ $\begin{array}{lll}-2.94714100 & 2.48060600 & 1.46776000\end{array}$ $-4.62051800-0.28731400-0.50475200$ $\begin{array}{llll}-3.30508900 & 0.28006800 & -1.55121900\end{array}$ $-3.14420600-1.25576000-0.67860400$

\section{Int-IIIB ( $\left.{ }^{t} \mathrm{BuOH}\right)$}

$H=-1038.134948$ au

$\begin{array}{lllll}\text { U } & 0.48389400 & 1.02585300 & -0.16328200\end{array}$

Si $\quad 0.74415200 \quad-2.51188800 \quad-0.83868100$

Si $\quad 3.30822800 \quad-0.76372800 \quad-0.96222600$

N $\quad \begin{array}{llll}1.58568700 & -0.96667200 & -0.67461900\end{array}$

C $\quad 3.82080300 \quad-1.15895700 \quad-2.74598900$

H $\quad 3.24956600 \quad-0.55007600 \quad-3.45418100$

$\mathrm{H} \quad 4.88540100-0.95056900-2.90183300$

H $\quad 3.64957000 \quad-2.21054100 \quad-2.99621700$

$\begin{array}{lllll}\text { C } & 4.41033000 & -1.73623200 & 0.23679900\end{array}$

H $\quad 4.35722600 \quad-2.81424500 \quad 0.06323200$

$\begin{array}{llll}H & 5.45692000 & -1.43174900 & 0.12148400\end{array}$

H $\quad 4.11547800 \quad-1.54512100 \quad 1.27373300$

$\begin{array}{llll}\text { C } & 3.70554700 & 1.08298500 & -0.66674000\end{array}$

H $\quad 3.17235000 \quad 1.76060800 \quad-1.34544000$

$\begin{array}{lllll}\mathrm{H} & 3.52289700 & 1.39975700 & 0.36796000\end{array}$

H $\quad 4.77361700 \quad 1.24169600 \quad-0.85317500$

C $\quad-0.29676600 \quad-2.52123800 \quad-2.42781500$

H $\quad-1.02960600 \quad-1.70672700 \quad-2.42953100$

H $\quad 0.34002500 \quad-2.40086700 \quad-3.31057800$

H $\quad-0.84891100 \quad-3.46171900-2.53590700$

C $\quad 1.88852500 \quad-4.02771400-0.89123700$

H $\quad 2.62962800 \quad-3.97945000 \quad-1.69478600$

H $\quad 2.41768200 \quad-4.16827300 \quad 0.05593600$

H $\quad 1.27783200-4.92165000-1.06355100$

$\begin{array}{lllll}\text { C } & -0.41314300 & -2.74384800 & 0.64788800\end{array}$

$\begin{array}{llll}\mathrm{H} & -0.99970900 & -3.66417500 & 0.54917800\end{array}$

$\begin{array}{llll}H & 0.15997700 & -2.80883500 & 1.57913700\end{array}$

$\mathrm{H} \quad-1.11653600 \quad-1.90927100 \quad 0.73548600$

$\begin{array}{lllll}\text { O } & 0.66873000 & 2.46949100 & -1.73932700\end{array}$

$\begin{array}{lllll}\text { C } & 0.69502800 & 3.46558200 & -2.73903400\end{array}$

$\begin{array}{lllll}\text { C } & 1.62936300 & 4.59384700 & -2.28829000\end{array}$ 


\begin{tabular}{|c|c|c|c|}
\hline & 1.20652500 & 2.83713000 & -4.04031600 \\
\hline & -0.72674000 & 4.00348500 & -2.93495300 \\
\hline & 1.27798400 & 5.02258700 & -1.34430900 \\
\hline & 2.64381800 & 4.21280600 & -2.13416300 \\
\hline & 1.67438300 & 5.39523100 & -3.0336780 \\
\hline & 0.54855900 & 2.01942500 & -4.35146000 \\
\hline & 1.24593000 & 3.57375000 & -4.85007300 \\
\hline & 2.21293300 & 2.43111000 & -3.89736200 \\
\hline & -0.75845400 & 4.77303200 & -3.713854 \\
\hline & -1.40168800 & 3.19232400 & -3.22629000 \\
\hline & -1.09828200 & 4.44360200 & -2.00385000 \\
\hline & -1.60769500 & 0.62515400 & 0.02370800 \\
\hline & -3.00663300 & 0.74947700 & 0.196559 \\
\hline & -3.38406100 & 0.17711900 & 1.56696100 \\
\hline & -3.38489500 & 2.23270700 & 0.12083500 \\
\hline & -3.70206200 & -0.03407200 & -0.92115 \\
\hline & -3.11209900 & -0.88086500 & 1.630116 \\
\hline & -2.85594600 & 0.71506900 & 2.36060600 \\
\hline & -4.46050600 & 0.26499500 & 1.74959700 \\
\hline & -3.09514900 & 2.65054500 & -0.84845200 \\
\hline & -4.46346400 & 2.37550200 & 0.24681700 \\
\hline & -2.87308300 & 2.79675500 & 0.90806600 \\
\hline & -4.79191000 & 0.02312300 & -0.82768300 \\
\hline & -3.42003800 & 0.36876400 & -1.89924600 \\
\hline & -3.40825800 & -1.08740400 & -0.885846 \\
\hline & 1.12185100 & 1.82647200 & 1.719562 \\
\hline & 1.44278300 & 2.39658200 & 2.9698500 \\
\hline & 0.15262000 & 2.55285700 & 3.78264000 \\
\hline & 2.41652800 & 1.46245600 & 3.69702500 \\
\hline & 2.09144200 & 3.76500200 & 2.73463300 \\
\hline & -0.55260100 & 3.20225700 & 3.25368100 \\
\hline & -0.32265900 & 1.57795300 & 3.93118000 \\
\hline & 0.35070600 & 2.99163200 & 4.76649000 \\
\hline & 3.33585300 & 1.34149600 & 3.1152560 \\
\hline & 2.68517500 & 1.85545600 & 4.68356600 \\
\hline & 1.96414700 & 0.47479500 & 3.83152500 \\
\hline & 2.35826600 & 4.24793100 & 3.6809450 \\
\hline & 3.00129400 & 3.65692000 & $2.135815 c$ \\
\hline & 1.40281400 & 4.42347400 & 2.195325 \\
\hline
\end{tabular}




\section{References}

(1) Andersen, R. A. Inorg. Chem. 1979, 18, 1507.

(2) Dormond, A.; El Bouadili, A.; Aaliti, A.; Moise, C. J. Organomet. Chem. 1985, 288, C1.

(3) Tietze, L. F.; Brasche, G.; Grube, A.; Böhnke, N.; Stadler, C. Chemistry - A European Journal 2007, $13,8543$.

(4) (a) Becke, A. D. J. Chem. Phys. 1993, 98, 5648; (b) Perdew, J. P.; Wang, Y. Phys. Rev. B: Condens. Matter 1992, 45, 13244.

(5) Moritz, A.; Cao, X.; Dolg, M. Theor Chem Acc 2007, 117, 473.

(6) Moritz, A.; Cao, X.; Dolg, M. Theor Chem Acc 2007, 118, 845.

(7) Bergner, A.; Dolg, M.; Küchle, W.; Stoll, H.; Preuß, H. Mol. Phys. 1993, 80, 1431.

(8) Ehlers, A. W.; Böhme, M.; Dapprich, S.; Gobbi, A.; Höllwarth, A.; Jonas, V.; Köhler, K. F.;

Stegmann, R.; Veldkamp, A.; Frenking, G. Chem. Phys. Lett. 1993, 208, 111.

(9) (a) Hariharan, P. C.; Pople, J. A. Theoret. Chim. Acta 1973, 28, 213; (b) Hehre, W. J.; Ditchfield, R.; Pople, J. A. J. Chem. Phys. 1972, 56, 2257.

(10) (a) Gonzalez, C.; Schlegel, H. B. J. Chem. Phys. 1989, 90, 2154; (b) Gonzalez, C.; Schlegel, H. B. J. Phys. Chem. 1990, 94, 5523.

(11) Frisch, M. J.; Trucks, G. W.; Schlegel, H. B.; Scuseria, G. E.; Robb, M. A.; Cheeseman, J. R.; Scalmani, G.; Barone, V.; Mennucci, B.; Petersson, G. A.; Nakatsuji, H.; Caricato, M.; Li, X.; Hratchian, H. P.; Izmaylov, A. F.; Bloino, J.; Zheng, G.; Sonnenberg, J. L.; Hada, M.; Ehara, M.; Toyota, K.; Fukuda, R.; Hasegawa, J.; Ishida, M.; Nakajima, T.; Honda, Y.; Kitao, O.; Nakai, H.; Vreven, T.; Montgomery Jr., J. A.; Peralta, J. E.; Ogliaro, F.; Bearpark, M. J.; Heyd, J.; Brothers, E. N.; Kudin, K. N.; Staroverov, V. N.; Kobayashi, R.; Normand, J.; Raghavachari, K.; Rendell, A. P.; Burant, J. C.; Iyengar, S. S.; Tomasi, J.; Cossi, M.; Rega, N.; Millam, N. J.; Klene, M.; Knox, J. E.; Cross, J. B.; Bakken, V.; Adamo, C.; Jaramillo, J.; Gomperts, R.; Stratmann, R. E.; Yazyev, O.; Austin, A. J.; Cammi, R.; Pomelli, C.; Ochterski, J. W.; Martin, R. L.; Morokuma, K.; Zakrzewski, V. G.; Voth, G. A.; Salvador, P.; Dannenberg, J. J.; Dapprich, S.; Daniels, A. D.; Farkas, Ö.; Foresman, J. B.; Ortiz, J. V.; Cioslowski, J.; Fox, D. J.; A.02 ed.; Gaussian, Inc.: Wallingford, CT, USA, 2009.

(12) Legault, C. Y. In CYLview; 1.0b ed. Universite de Sherbrooke, 2009. 